Técnicas de Bifurcação para o Problema de Yamabe em Variedades com Bordo

\author{
Ana Cláudia da Silva Moreira
}

TESE APRESENTADA

$\mathrm{AO}$

Instituto De Matemática e Estatística

DA

Universidade De SÃo Paulo

PARA

OBTENÇÃO DO TÍTULO

$\mathrm{DE}$

DOUTORA EM CIÊNCIAS

Programa: Doutorado em Matemática

Orientador: Prof. Dr. Paolo Piccione 


\section{Técnicas de Bifurcação para o Problema de Yamabe em Variedades com Bordo}

\footnotetext{
Esta é a versão final da Tese que contém as correções e alterações sugeridas pela Comissão Julgadora durante a defesa da versão original do trabalho, realizada em 29/01/2016. Uma cópia da versão original está disponível no Instituto de Matemática e Estatística da Universidade de São Paulo.
}

Comissão Julgadora:

- Prof. Dr. Paolo Piccione (orientador) - IME - USP

- Prof. Dr. Francesco Mercuri - IMECC - Unicamp

- Prof. Dr. Carlos Eduardo Durán Fernandez - UFPR

- Prof. Dr. Fabiano Brito - IME - USP

- Prof. Dr. Adriano João da Silva - IMECC - Unicamp 


\section{Agradecimentos}

A teacher affects eternity; he can never tell where his influence stops.

- Henry Brooks Adams -

Sou grata a muitas pessoas incríveis que me apoiaram, incentivaram e estiveram comigo durante todos esses anos que tenho estudado matemática, desde o professor José Adonai Pereira Seixas (UFAL), que foi o responsável por despertar em mim o desejo de estudar matemática até meu orientador de doutorado, professor Paolo Piccione, com quem aprendi a naturalidade de não saber, a confiança na capacidade de aprender e a tolerância no processo que liga um estado ao outro.

Já que encerro uma longa fase como aluna, quero aproveitar a oportunidade para dizer que sempre me lembro das excelentes aulas ministradas pelos professores Jorge Mujica (Unicamp), José Luiz Boldrini (Unicamp) e André Oliveira Gomes (IME-USP), e de suas didáticas impecáveis.

Durante esse processo de formação no qual somos, inevitavelmente, pressionados por prazos e avaliações, o papel de pesquisadores entusiasmados é mais importante do que se imagina. Aqui no IME, o professor Marcos Martins Alexandrino da Silva, e meu próprio orientador, sempre alimentaram em mim aquela energia que nos impulsiona a estudar matemática. Na Unicamp, meu orientador de mestrado, Carlos Eduardo Durán Fernandez (hoje na UFPR), desempenhou muito bem este papel e até hoje, sua sempre disponível atenção é motivadora. Neste mesmo sentido, quero dar destaque a uma homenagem mais que especial ao professor Alcibiades Rigas (Unicamp), por quem tenho muito carinho e admiração. Sua influência foi decisiva no meu encantamento e escolha pela Geometria. Inesquecível, ele será sempre uma inspiração para mim.

Mas, nem só de inspiração se faz um matemático. Os recursos materiais são necessários. Meu pai, não só apoiou minha decisão de voltar a estudar aos 26 anos, como me socorreu, muitas vezes, financeiramente. Nunca vou me esquecer das oportunidades de estágios que a professora Sueli Irene Rodrigues Costa (Unicamp) me ofereceu em momentos de dificuldade durante o curso de graduação. Sem essa ajuda não sei se teria sido possível chegar até aqui. Serei eternamente grata.

Agradeço à colaboração do colega Elkin Dario Cárdenas Diaz no artigo cujos resultados compõem uma parte desta Tese (Seção 2.3) e à minha amiga Luciana Carrara Abreu, que me ajudou com algumas das figuras utilizadas.

Por fim, agradeço ao meu marido, Robson da Silva, professor de matemática da Unifesp, em São José dos Campos, que desde o dia que decidi seguir a carreira de matemática, esteve ao meu lado, incentivando-me e dando suporte. Em particular, durante o doutorado, ele foi também minha fonte financiadora, diante da escassez de bolsas. Ao longo desses anos, sei que muitas vezes, ele sacrificou seus próprios planos para permitir que eu realizasse o sonho de um dia me tornar uma 
pesquisadora.

Ao final desta etapa aumenta a certeza de quão pouco sei. Entendi que natural é não saber. Aprender é um processo (diferente para cada indivíduo). Respeitar os limites do outro é reconhecer sua própria imperfeição. Conhecer sua capacidade é necessário para saber aproveitá-la. É você quem define o seu valor, ninguém mais. Respeitar os próprios limites é amar a si mesmo. O tempo é um elemento essencial na maturação do conhecimento. Na Matemática, haverá sempre mais para se aprender, descobrir e criar. E que seja belo! 


\section{Resumo}

MOREIRA, A. C. S. Técnicas de Bifurcação para o Problema de Yamabe em Variedade com Bordo. 2016. 125 pg. Tese (Doutorado) - Instituto de Matemática e Estatística, Universidade de São Paulo, São Paulo, 2016.

Apresentaremos alguns resultados de rigidez e de bifurcação para soluções do problema de Yamabe em variedades produto com bordo.

Palavras-chave: geometria Riemanniana, problema de Yamabe e suas generalizações, teoria de bifurcação, ações isométricas. 


\section{Abstract}

MOREIRA, A. C. S. Bifurcation Techniques for the Yamabe Problem in Manifolds with

Boundary. 2016. 125 pg. Tese (Doutorado) - Instituto de Matemática e Estatística, Universidade de São Paulo, São Paulo, 2016.

We will discuss some rigidity and bifurcation results for solutions of the Yamabe Problem in product manifolds with boundary.

Keywords: Riemannian geometry, Yamabe problem and its generalizations, bifurcation theory, isometric actions. 


\section{Sumário}

$\begin{array}{ll}\text { Introdução } & 1\end{array}$

$\begin{array}{lll}\text { I } & \text { Preliminares } & 7\end{array}$

1 Geometria Riemanniana $\quad 9$

1.1 Sistemas de Coordenadas em Variedades Riemannianas . . . . . . . . . . . . . 11

1.2 Variedades com Bordo . . . . . . . . . . . . . . . . . . . . . . . . . 12

1.3 Tensores em Geometria Riemanniana . . . . . . . . . . . . . . . . . . . 13

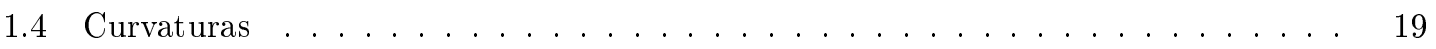

1.5 Transformações Conformes . . . . . . . . . . . . . . . . . . 24

1.5.1 Conexão Riemanniana . . . . . . . . . . . . . . . . . . . . 25

1.5 .2 Curvatura Riemanniana . . . . . . . . . . . . . . . . . 27

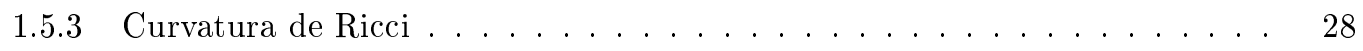

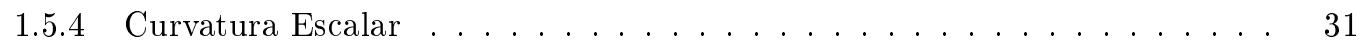

1.5.5 Curvatura Média . . . . . . . . . . . . . . . . . . 32

1.5 .6 Operador Laplaciano . . . . . . . . . . . . . . . . . . . 33

$1.5 .7 \quad$ Variedade Produto . . . . . . . . . . . . . . . . . . . . 34

2 Análise Funcional $\quad 41$

2.1 Operadores de Fredholm . . . . . . . . . . . . . . . . . . . 44

2.2 Espaços de Hölder . . . . . . . . . . . . . . . . . . . . . 45

3 Representações de Grupos de Lie $\quad 49$

3.1 Ações Isométricas e Representações . . . . . . . . . . . . . . . . . . . . . . . . 51

II Técnicas de Bifurcação Aplicadas ao Problema de Yamabe $\quad 55$

1 Teoremas de Bifurcação Variacional $\quad 57$

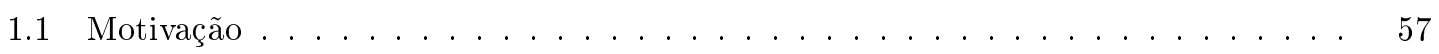

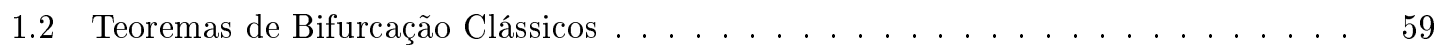

1.3 Teoremas de Bifurcação com Domínio Variável . . . . . . . . . . . . . 60 
2 Bifurcação de Soluções para o Problema de Yamabe 65

2.1 O Problema de Yamabe . . . . . . . . . . . . . . . . . 65

2.2 Bifurcação de Soluções em Variedades Produto . . . . . . . . . . . . . . . . 66

2.3 Bifurcação de Soluções em Variedades Produto com Bordo Mínimo . . . . . . . . . 68

2.3.1 Variedades e Classes Conformes . . . . . . . . . . . . . . 69

2.3.2 Rigidez Local e Bifurcação de Soluções do Problema de Yamabe . . . . . 76

2.3.3 Bifurcação de Soluções para o Problema de Yamabe em Variedades Produto com Bordo Mínimo . . . . . . . . . . . . . . . . . . 78

3 Bifurcação Equivariante de Soluções para Problema de Yamabe 85

3.1 Bifurcação Equivariante em Variedades com Bordo Mínimo . . . . . . . . . . . . . 85

3.2 Bifurcação Equivariante de Soluções do Problema de Yamabe em Variedades Produto com Bordo Mínimo . . . . . . . . . . . . . . . . . . . . 92

$\begin{array}{lll}\text { III Apêndice } & 99\end{array}$

$\begin{array}{lr}\text { A O Funcional de Hilbert-Einstein } & 101\end{array}$

A.1 Primeira Variação do Funcional de Hilbert-Einstein . . . . . . . . . . . . . . . . . . 101

A.2 Segunda Variação do Funcional de Hilbert-Einstein . . . . . . . . . . . . . . . . 109

B Funções com Derivada Normal Prescrita 115

$\begin{array}{lr}\text { C G-equivariância da Curvatura Escalar } & 121\end{array}$

$\begin{array}{lr}\text { Bibliografia } & \mathbf{1 2 2}\end{array}$ 


\section{Introdução}

Problemas relacionados à obtenção de métricas canônicas em uma variedade riemanniana têm ocupado a mente de geômetras há vários anos. Dada uma variedade riemanniana $(M, g)$, a $\mathcal{C}^{k}$-classe conforme da métrica $g$ é dada por

$$
[g]=\left\{\phi g: \phi \in \mathcal{C}_{+}^{k}(M)\right\}
$$

Provavelmente, o resultado mais conhecido neste assunto seja o Teorema de Uniformização para superfícies simplesmente conexas que garante a existência de uma métrica de curvatura gaussiana constante em cada classe conforme. Em dimensões mais altas, outras noções de curvatura - seccional, de Ricci, escalar - trazem diferentes informações sobre a variedade. Poderíamos nos perguntar se é sempre possível encontrar métricas conformes que tornam tais curvaturas constantes. Para a curvatura seccional, não existe resultado semelhante, variedades riemannianas com curvatura seccional constante constituem uma classe especial de variedades chamada de espaço forma. Uma variedade que tem curvatura de Ricci constante é conhecida como variedade de Einstein. No caso da curvatura escalar, o matemático japonês Hidehiko Yamabe (1923-1960) propôs uma conjectura que ficou conhecida como Problema de Yamabe:

Seja $(M, g)$ uma variedade riemanniana compacta de dimensão $m \geq 3$. Existe uma métrica $\tilde{g}$ na classe conforme de $g$, segundo a qual $M$ tem curvatura escalar constante?

Seja $\tilde{g} \in[g]$, dada por $\tilde{g}=u^{\frac{4}{m-2}} g$, onde $u \in \mathcal{C}_{+}^{k}(M)$. Calculando a curvatura escalar com respeito à métrica $\tilde{g}$, temos

$$
R_{\tilde{g}}=u^{-\frac{m+2}{m-2}}\left(\frac{4(m-1)}{m-2} \Delta_{g} u+R_{g} u\right),
$$

onde $\Delta_{g}$ é o operador laplaciano em $M$ com respeito à métrica $g$ e $R_{g}$ é a curvatura escalar. Então o problema de Yamabe tem solução se existe $u>0$ que satisfaz a E.D.P.

$$
\Delta_{g} u+\frac{m-2}{4(m-1)} R_{g} u=K u^{\frac{m+2}{m-2}}
$$

para $K=\frac{(m-2)}{4(m-1)} R_{\tilde{g}}$ constante.

O problema tem um equivalente variacional. Seja $\mathcal{M}^{k}(M)$ a variedade de Banach das métricas riemannianas em $M$, que é um cone aberto no espaço dos $(0,2)$-tensores simétricos de classe $\mathcal{C}^{k}$ definidos em $M, \Gamma^{k}\left(T^{*} M \otimes T^{*} M\right)$. A $\mathcal{C}^{k}$-classe conforme $[g]$ é uma subvariedade de $\mathcal{M}^{k}(M)$ e $T_{g}[g]=\left\{\psi g: \psi \in \mathcal{C}^{k}(M)\right\}$. Yamabe percebeu que a equação (1) é a equação de Euler-Lagrange para o funcional de Hilbert-Einstein normalizado $F: \mathcal{M}^{k}(M) \longrightarrow \mathbb{R}$, suave em $\mathcal{M}^{k}(M)$ e em $[g]$, dado por

$$
F(g)=\frac{\int_{M} R_{g} \omega_{g}}{\mathcal{V}_{g}^{\frac{m-2}{m}}}, \quad \text { com } \quad \mathcal{V}_{g}=\mathcal{V}(g)=\int_{M} \omega_{g},
$$


restrito à classe conforme de $g$ :

$$
u \text { é solução da } \operatorname{EDP}(1) \Longleftrightarrow u \text { é ponto crítico de } F_{g}(u)=\left.F\right|_{[g]}\left(u^{\frac{4}{m-2}} g\right) \text {. }
$$

Em 1960, foi publicado o artigo [41], atribuído à Yamabe, que trazia uma prova para a conjectura. Mas, em 1968, o matemático australiano NeilTrudinger publicou o artigo [39], onde apontava um problema na demonstração de Yamabe. É claro que esse fato não desmerece o trabalho de Yamabe, afinal tratava-se de um problema tão difícil que entreteve os matemáticos por cerca de 16 anos. Só em 1984, o esforço combinado de Aubin [2], Trudinger [39], e Schoen [34], [35] forneceu uma prova definitiva da existência de solução para o problema. Foram usadas técnicas de geometria diferencial, análise funcional e equações diferenciais parciais.

Hoje sabemos que os pontos críticos do funcional

$$
F(g)=\int_{M} R_{g} \omega_{g}
$$

em $\mathcal{M}^{k}(M)$, são as métricas $g$ tais que $\operatorname{Ric}_{g}=0$. Se considerarmos a restrição de $F$ à subvariedade das métricas riemannianas de volume 1, denotada por $\mathcal{M}^{k}(M)_{1}$, os pontos críticos são as métricas de Einstein de volume 1. Restringindo o funcional à classe conforme de volume 1 ,

$$
[g]_{1}=\left\{\phi g: \phi \in \mathcal{C}_{+}^{k}(M), \int_{M} \phi^{\frac{m}{2}} \omega_{g}=1\right\}
$$

que é subvariedade de $\mathcal{M}^{k}(M)_{1}$ e cujo espaço tangente é $T_{g}[g]_{1}=\left\{\psi g: \psi \in \mathcal{C}^{k}(M), \int_{M} \psi \omega_{g}=0\right\}$, a primeira variação do funcional $F$ toma a forma

$$
\delta F(g) h=-\int_{M}\left\langle R i c_{g}-\frac{R_{g}}{2} g, h\right\rangle_{g} \omega_{g},
$$

para $g \in \mathcal{M}^{k}(M)$ e $h \in T_{g} \mathcal{M}^{k}(M)$, e os pontos críticos de $F$ em $[g]_{1}$ são as métricas conformes a $g$, de volume 1 e $R_{g}$ constante.

O Problema de Yamabe deu origem a uma coleção de trabalhos concernentes à existência, unicidade e outros aspectos de suas soluções. Em particular Lima, Piccione e Zedda [31] estudaram rigidez local e multiplicidade de métricas de curvatura escalar constante nas classes conformes, em variedades produto compactas fazendo uso de teoria de bifurcação. Inspirados neste trabalho, estudamos o problema para o caso de variedades com bordo.

O Problema de Yamabe no caso de variedades com bordo tem diferentes formulações. Seja $(M, \bar{g})$ uma variedade riemanniana compacta com bordo $\partial M \neq \emptyset, \operatorname{dim} M=m \geq 3$. Questiona-se a existência de $\tilde{g} \in[\bar{g}]$ tal que

(1) $R_{\tilde{g}}$ é constante não nula e $H_{\tilde{g}}=0$,

(2) $R_{\tilde{g}}=0$ e $H_{\tilde{g}}$ é constante não nula,

(3) $R_{\tilde{g}}$ e $H_{\tilde{g}}$ são ambas constantes não nulas,

onde $H_{\tilde{g}}$ é a curvatura média do bordo. A existência de solução para estes problemas foi provada por Cherrier [12], Escobar [18], [19], [20], Almaraz [1], Han [23] e outros, para uma vasta classe de variedades com bordo. Nesta tese estudamos a formulação (1): $R_{\tilde{g}}$ constante e $H_{\tilde{g}}=0$, para a qual temos o seguinte problema:

$$
\left\{\begin{array}{l}
\frac{4(m-1)}{m-2} \Delta_{\bar{g}} u+R_{\bar{g}} u-K u^{\frac{m+2}{m-2}}=0, \text { em } M \\
\partial_{\eta} u+\frac{n-2}{2} H_{\bar{g}} u-L u^{\frac{n}{n-2}}=0, \quad \text { em } \partial M
\end{array}\right.
$$


onde $K$ e $L$ são constantes e $\eta$ é o campo unitário (interior) normal ao bordo, que é uma espécie de problema não-linear de autovalor. Este problema tem também uma estrutura variacional. Definimos a $\mathcal{C}^{k, \alpha}$-classe conforme normalizada ${ }^{1}$ da métrica $\bar{g}$ por

$$
[\bar{g}]^{0}=\left\{\tilde{g} \in[\bar{g}]: H_{\tilde{g}}=0\right\}
$$

Observe que, fazendo $\tilde{g}=u^{\frac{4}{m-2}} \bar{g} \in[\bar{g}]$, temos

$$
H_{\tilde{g}}=\phi^{-\frac{m}{m-2}}\left(H_{\bar{g}} \phi+\frac{2(m-1)}{(m-2)} \partial_{\eta_{\bar{g}}} \phi\right),
$$

então, podemos identificar $[\bar{g}]^{0}$ com o conjunto

$$
\mathcal{C}_{+}^{k, \alpha}(M)^{0}=\left\{\phi \in \mathcal{C}_{+}^{k, \alpha}(M): \partial_{\eta_{\bar{g}}} \phi=0 \text { em } \partial M\right\}
$$

Nesta tese, provamos que $[\bar{g}]^{0}$ é subvariedade de $[\bar{g}]$ e

$$
T_{\bar{g}}[\bar{g}]^{0}=\left\{\psi \bar{g}: \psi \in \mathcal{C}^{k, \alpha}(M), \partial_{\eta_{\bar{g}}} \psi=0\right\}
$$

o que nos permite calcular os pontos críticos do funcional $F$, restrito à classe conforme normalizada de volume 1 ,

$$
[\bar{g}]_{1}^{0}=[\bar{g}]^{0} \cap \mathcal{M}^{k, \alpha}(M)_{1},
$$

que é uma subvariedade de $\mathcal{M}^{k, \alpha}(M)_{1}$, e cujo espaço tangente é dado por

$$
T_{\bar{g}}[\bar{g}]_{1}^{0}=\left\{\psi \bar{g}: \psi \in \mathcal{C}^{k, \alpha}(M), \partial_{\eta_{\bar{g}}} \psi=0, \int_{M} \psi \omega_{\bar{g}}=0\right\} .
$$

Na primeira variação do funcional Hilbert-Einstein em variedades com bordo $\partial M \neq \emptyset$, aparece uma integral sobre o bordo,

$$
\delta F(\bar{g}) h=-\int_{M}\left\langle R i c_{\bar{g}}-\frac{R_{\bar{g}}}{2} \bar{g}, h\right\rangle_{\bar{g}} \omega_{\bar{g}}-2 \int_{\partial M}\left(\delta H_{\bar{g}}+\frac{1}{2}\left\langle I I_{\bar{g}}, h\right\rangle\right) \sigma_{\bar{g}},
$$

para todo $h \in T_{\bar{g}} \mathcal{M}^{k, \alpha}(M)$, que acaba sendo anulada quando restringimos $F$ à classe conforme normalizada $[\bar{g}]^{0}$, pois ao fazermos $h=\psi \bar{g} \in T_{\bar{g}}[\bar{g}]^{0}$, teremos termos como o traço da segunda forma fundamental e derivadas na direção normal ao bordo que são todos nulos. Assim, depois de mais algumas contas, podemos concluir que $\bar{g}$ é ponto crítico de $\left.F\right|_{[\bar{g}]_{1}^{0}}$, se e só se $\bar{g} \in[\bar{g}]_{1}^{0}$ e $R_{\bar{g}}$ é constante.

Agora nos interesse estudar a natureza de tais pontos críticos. Assim, se $\bar{g}$ é um ponto crítico de $F$ restrito à $[\bar{g}]_{1}^{0}$, calculamos a segunda variação do funcional

$$
\delta^{2} F(\bar{g})(\psi, \psi)=\frac{m-2}{2} \int_{M}\left[(m-1) \Delta_{\bar{g}} \psi-R_{\bar{g}} \psi\right] \psi \omega_{\bar{g}}
$$

onde $\psi \in \mathcal{C}^{k, \alpha}(M)^{0}$ e tem integral nula. Utilizando propriedades especiais do laplaciano em espaços de Holder e características inerentes aos operadores Fredholm, estudamos as condições de degeneração deste ponto e estabelecemos a seguinte definição.

Definição: Seja $\bar{g} \in \mathcal{M}^{k, \alpha}(M)_{1}$ com curvatura escalar constante $R_{\bar{g}}$ em $M$. Dizemos que $\bar{g} e ́$

\footnotetext{
${ }^{1}$ Por razões técnicas, que ficarão claras ao longo do texto, trabalhamos com os Espaços de Holder

$$
\mathcal{C}^{k, \alpha}=\left\{\phi \in \mathcal{C}^{k}(M):\left|D^{a} \phi(x)-D^{a} \phi(y)\right| \leq L|x-y|^{\alpha}, \forall x, y \in M, x \neq y, \forall a \in \mathcal{A}_{k}\right\},
$$
}

onde $\alpha \in(0,1], L$ é uma constante, $\mathcal{A}_{k}=\left\{a=\left(a_{1}, \ldots, a_{n}\right): a_{i} \geq 0, \sum_{i=1}^{n} a_{i}=k\right\}$. 
uma métrica não degenerada se $R_{\bar{g}}=0$ ou se $\frac{R_{\bar{g}}}{(m-1)}$ não é um autovalor de $\Delta_{\bar{g}}$, com condição de Neumann no bordo $\partial_{\eta_{\bar{g}}} f=0$. Em outras palavras, $\frac{R_{\bar{g}}}{(m-1)}$ não é uma solução do problema de autovalor

$$
\left\{\begin{aligned}
\Delta_{\bar{g}} f & =\lambda f, \text { em } M \\
\partial_{\eta_{\bar{g}}} f & =0, \text { em } \partial M
\end{aligned}\right.
$$

Então, o resultado de rigidez local de [30] se estende naturalmente para o problema de Yamabe em variedades com bordo na formulação (1): se $\bar{g}$ é uma métrica não degenerada de volume 1, curvatura escalar constante e curvatura média do bordo nula, há unicidade local de soluções para o problema de Yamabe na classe conforme normalizada de $\bar{g}$.

A partir deste ponto, podemos dividir esta tese em dois trabalhos distintos. O primeiro, exposto na Seção 2.3, que deu origem ao artigo [14], a ser publicado no periódico Advances in Nonlinear Analysis. Neste trabalho, adaptamos o Teorema de Bifurcação de [31], que garante a existência de um instante de bifurcação em um determinado intervalo, baseado no salto do índice de Morse da família de operadores

$$
\mathcal{J}_{s}=\Delta_{\bar{g}_{s}}-\frac{R_{\bar{g}_{s}}}{m-1} I
$$

à medida que $s$ varia, para o caso de variedades com bordo. Além disso, estendemos os resultados obtidos no estudo de multiplicidade de soluções para o problema de Yamabe em variedades produto para o caso com bordo. Na situação estabelecida abaixo:

Seja $\left(M_{1}, g^{(1)}\right)$ uma variedade riemanniana compacta com $\partial M_{1}=\emptyset$ e curvatura escalar constante, e seja $\left(M_{2}, \bar{g}^{(2)}\right)$ uma variedade riemanniana compacta com bordo mínimo e curvatura escalar constante. Considere a variedade produto, $M=M_{1} \times M_{2}$, cujo bordo é dado por $\partial M=M_{1} \times \partial M_{2}$. Sejam $m_{1}$ e $m_{2}$ as dimensões de $M_{1}$ e $M_{2}$, respectivamente, e assuma que $\operatorname{dim}(M)=m=$ $m_{1}+m_{2} \geq 3$. Para cada $s \in(0,+\infty)$, defina $\bar{g}_{s}=g^{(1)} \oplus s \bar{g}^{(2)}$ uma família de métricas em $M$. Então, $\left\{\bar{g}_{s}\right\}_{s} \subset \mathcal{M}^{k, \alpha}(M)$ é uma familia de soluções para o Problema de Yamabe na variedade com bordo $M$.

Pergunta-se se em algum instante $s$ há multiplicidade de soluções para o problema de Yamabe na classe conforme $\left[\bar{g}_{s}\right]_{1}^{0}$.

Tendo em vista a definição anterior, observe que se $\bar{g}_{s}$ é degenerado, então $s$ é um candidato a instante de bifurcação. Provamos que os resultados de [31] se mantém no caso com bordo, isto é, exceto por um número finito de instantes, todos os demais $s$, tais que $\bar{g}_{s}$ é degenerado, são instantes de bifurcação.

A teoria de bifurcação contribui para a solução do problema de forma direta: dada uma família contínua $\left\{\bar{g}_{s}\right\}_{s>0}$ de soluções para o problema de Yamabe na variedade produto, construída como estabelecido acima, encontrar um instante de bifurcação $s_{*}$ significa que existem sequências $s_{n} \rightarrow s_{*}$ e $\bar{g}_{n} \rightarrow \bar{g}_{s_{*}}$ tais que cada $\bar{g}_{n}$ é uma solução do problema estudado, na classe conforme $\left[\bar{g}_{s_{n}}\right]_{1}^{0}$, distinta de $\bar{g}_{s_{n}}$.

O segundo trabalho, objeto do Capítulo 3, traz o estudo de bifurcação equivariante, que consiste em considerar a ação, via isometrias, de um grupo de Lie conexo, de dimensão finita, no espaço funcional do problema que deixa invariante o conjunto de soluções.

Neste trabalho, provamos uma extensão natural do Teorema de Bifurcação Equivariante, de [31], para variedades com bordo. Como os autoespaços de $\Delta_{\bar{g}_{s}}$ são $G$-invariantes, a ação isométrica de $G$ determina antirrepresentações em todos os autoespaços do laplaciano. A abordagem equivariante permite o uso de um critério mais fino para garantir a existência de instantes de bifurcação, qual seja, a análise das representações do grupo $G$ nos autoespaços do laplaciano, já que, nem sempre é possível detectar um salto no índice de Morse, .

De posse dos critérios para existência de um ramo de bifurcação, estudamos o problema de 
multiplicidade de soluções para o problema de Yamabe em variedades produto. Propusemos uma outra definição para ação harmonicamente livre, diferente daquela sugerida em [31].

Definição: Seja $G$ um grupo de Lie que age por isometrias em uma variedade riemanniana $(N, h)$. A ação de $G$ é dita harmonicamente livre se, dada uma família arbitrária de autoespaços do laplaciano $\Delta_{h}$, dois a dois distintos,

$$
V_{1}, V_{2}, \ldots, V_{r}, V_{r+1}, \ldots, V_{r+s}, \quad r, s \geq 1
$$

e dados inteiros $n_{\ell} \geq 0$, com $\ell=1, \ldots, r+s$, não todos nulos, então as antirrepresentações

$$
\bigoplus_{\ell=1}^{r} n_{\ell} \cdot \pi_{\ell} \quad e \quad \bigoplus_{\ell=r+1}^{r+s} n_{\ell} \cdot \pi_{\ell}
$$

são não-equivalentes. Aqui, denotamos por $\pi_{\ell}$ a antirrepresentação de $G$ em $V_{\ell}$, definida na Proposição 3.1.2, e $n_{\ell} \cdot \pi_{\ell}$ denota a soma direta (externa) de $n_{\ell}$ cópias de $\pi_{\ell}$.

Com base nesta nova definição, considerando a ação harmonicamente livre de um grupo de Lie conexo, via isometrias, em um dos fatores da variedade produto $M=M_{1} \times M_{2}$, temos uma ação de $G$ sobre $M$, dada por

$$
\begin{array}{lll}
G \times M & \longrightarrow & M \\
(\xi,(x, y)) & \longmapsto & (\xi x, y)
\end{array},
$$

que determina antirrepresentações nos autoespaços $V_{i}^{(1)}$ e $V_{j}^{(2)}$ de $\Delta_{g^{(1)}}$ e de $\Delta_{\bar{g}^{(2)}}$, respectivamente. Consequentemente, temos antirrepresentações nos autoespaços $V_{i, j}=V_{i}^{(1)} \otimes V_{j}^{(2)}$ de $\mathcal{J}_{s}$,

$$
\pi_{i, j}(\xi)(f)=\left(f_{1} \circ \xi\right) \otimes f_{2} .
$$

Se $s_{*}$ é um instante de degeneração para a família $\left\{\bar{g}_{s}\right\}_{s>0}$, provamos que as antirrepresentações de $G$ nos autoespaços negativos de $\mathcal{J}_{s_{*}-\varepsilon}$ e $\mathcal{J}_{s_{*}+\varepsilon}$ são não equivalentes. Assim, segue do Teorema de Bifurcação Equivariante que todo instante de degeneração é um instante de bifurcação. 


\section{Parte I}

\section{Preliminares}





\section{Capítulo 1}

\section{Geometria Riemanniana}

Este capítulo tem o intuito de recordar, brevemente, alguns conceitos importantes em geometria riemanniana.

Definição 1.0.1. Uma variedade diferenciável $M$, de dimensão $m$, é um espaço topológico (Hausdorff, com base enumerável) com uma dada estrutura diferencial, isto é, uma coleção de cartas $\mathcal{A}=\left\{\left(U_{i}, \phi_{i}\right)\right\}$, chamada atlas, onde $\phi_{i}$ é um homeomorfismo entre $U_{i}$ e algum aberto de $\mathbb{R}^{n}$, para todo $i$, tal que

- $M=\bigcup_{i} U_{i}$,

- para cada par de cartas $(U, \phi),(V, \psi), U \cap V \neq \emptyset$, a aplicação $\psi \circ \phi^{-1}: \phi(U \cap V) \rightarrow \psi(U \cap V)$ é diferenciável.

Dizemos que uma variedade $M$ é orientável se possui um atlas orientado no sentido que o determinante Jacobiano da mudança de coordenadas $\psi \circ \phi^{-1}$ é positivo para todo par de cartas $(U, \phi),(V, \psi)$ deste atlas, em cada ponto da interseção $\phi(U \cap V)$.

Denotaremos por $\mathcal{C}^{\infty}(M)$ o espaço de todas as funções reais, infinitamente diferenciáveis, definidas em $M ; T_{p} M$ é o espaço tangente a $M$ no ponto $p \in M$ e $T M$ denota o fibrado tangente, isto é, a união disjunta dos espaços tangentes para todo $p \in M$. Um campo diferenciável em uma variedade $M$ é uma aplicação que associa a cada $p \in M$ um vetor $X_{p} \in T_{p} M$ e varia suavemente com $p$; denotamos por $\mathfrak{X}(M)$ o conjunto dos campos diferenciáveis em $M$. Mais geralmente, uma seção suave de um fibrado $E$ sobre $M$ é uma aplicação diferenciável $\xi$ que associa a cada ponto $p \in M$ um ponto $\xi(p)$ na fibra $E_{p} \subset E$.

Seja $f: N^{n} \longrightarrow M^{m}$ uma aplicação diferenciável entre variedades.

(i.) Se $(\mathrm{d} f)_{p}: T_{p} N \longrightarrow T_{f(p)} M$ é injetor, para todo $p \in N$, dizemos que $f$ é uma imersão.

(ii.) Se $f$ é uma imersão e é injetora, então $f(N)$, com a estrutura diferenciável que faz com que $f$ seja um difeomorfismo (herdada de $N$ ), é chamada subvariedade imersa de $M$.

(iii.) Se a topologia herdada de $N$ coincidir com a topologia induzida por $M$ em $f(N)$ (a topologia do subespaço) dizemos que $f(N)$ é uma subvariedade mergulhada de $M$.

(iv.) $f$ é chamada submersão se $(\mathrm{d} f)_{p}: T_{p} N \longrightarrow T_{f(p)} M$ é sobrejetor.

(v.) Dizemos que $f$ é um mergulho se $f$ é uma imersão e um homeomorfismo sobre a imagem, $f(N)$, na topologia induzida por $M$. Se $f$ é um mergulho, $f(N)$ é chamada subvariedade mergulhada de $M$.

Definição 1.0.2. Seja $M$ uma variedade diferenciável de dimensão $m$. Um subconjunto $N \subset M$ é uma subvariedade (regular), de dimensão $n$, da variedade $M$, se para todo $p \in N$, existe uma carta $(U, \phi)$ de $M$, com $p \in U$, tal que $\phi(U \cap N)=\phi(U) \cap \mathbb{R}^{n} \times\{0\}$. 
Observe que uma subvariedade (diferenciável) imersa de dimensão $n$ de $M$ é um subconjunto $S \subset M$ munido de uma topologia de $n$-variedade e uma estrutura diferenciável tal que a inclusão $i: S \hookrightarrow M$ é uma imersão. Se, além disso, a topologia em $S$ é a topologia induzida por $M$, então a inclusão é um mergulho e $S$ é uma subvariedade mergulhada de $M$. Subvariedades mergulhadas também são chamadas de subvariedades regulares.

Considerando ainda uma aplicação diferenciável $f: M^{m} \longrightarrow N^{n}$, entre variedades diferenciáveis, um ponto $q \in N$ é dito um valor regular para $f$, se $(\mathrm{d} f)_{p}$ é sobrejetivo para todo $p \in f^{-1}(q)$. Segue diretamente da Forma Local das Submersões o seguinte resultado.

Teorema 1.0.3. Se q é um valor regular de $f: M^{m} \longrightarrow N^{n}, n \leq m$, então $f^{-1}(\{q\})$ é uma subvariedade regular de $M$, com $\operatorname{dim} f^{-1}(q)=\operatorname{dim} M-\operatorname{dim} N$. Além disso, $\operatorname{ker}(d f)_{p}=T_{p}\left(f^{-1}\{q\}\right)$ para todo $p \in f^{-1}\{q\}$.

Definição 1.0.4. Dizemos que $f: M^{m} \longrightarrow N^{n}$ é transversal à subvariedade $Y \subset N$ se

$$
\left(d f_{x}\right)\left(T_{x} M\right)+T_{y}(Y)=T_{y}(N)
$$

com $y=f(x)$, para todo $x \in f^{-1}(Y)$. Neste caso, $f^{-1}(Y)$ é subvariedade (regular) de $M$ e a codimensão de $f^{-1}(Y)$ em $M$ é igual à codimensão de $Y$ em $N$.

Em particular, quando $Y=\{y\}, f$ é transversal a $Y$, se $\mathrm{d} f_{x}\left(T_{x} M\right)=T_{y} N$, para todo $x \in$ $f^{-1}(Y)$, isto é, o diferencial é sobrejetor e $y$ é um valor regular.

Agora, sejam $X$ e $Y$ subvariedades de uma variedade diferenciável $M$. E considere as inclusões $i_{X}: X \hookrightarrow M$ e $i_{Y}: Y \hookrightarrow M$. Observe que $x \in X$ pertence a imagem inversa de $Y$ pela inclusão se e só se $x \in X \cap Y$, além disso, $\left(\mathrm{d} i_{X}\right)_{x}$ é simplesmente a inclusão de $T_{x} X$ em $T_{x} M$ e o mesmo vale para $i_{Y}$. Segue diretamente da definição que a inclusão $i_{X}$ é transversal à subvariedade $Y$ de $M$ se e somente se, para todo $x \in X \cap Y$, temos

$$
T_{x} X+T_{x} Y=T_{x} M
$$

o mesmo vale para $i_{Y}$ com respeito à subvariedade $X$. Neste caso dizemos que $X$ é transversal à $Y$ e denotamos por $X \pitchfork Y$. A interseção de duas subvariedades transversais de $M$ é uma uma subvariedade de $M$. Além disso, $\operatorname{codim}(X \cap Y)=\operatorname{codim} X+\operatorname{codim} Y$.

O espaço dual a $T_{p} M$, denotado por $T_{p}^{*} M$, é chamado espaço cotangente a $M$ em $p$ e é o espaço de todos os funcionais lineares definidos em $T_{p} M$, chamados vetores cotangentes.

Se $f \in \mathcal{C}^{\infty}(M)$, a aplicação $X_{p} \mapsto X_{p}(f)$ está definida para todo vetor $X_{p} \in T_{p} M$. Se $\left(x_{1}, \ldots, x_{n}\right)$ é um sistema de coordenadas em uma vizinhança de $p \in M$, as derivadas parciais $\partial_{i}=\frac{\partial}{\partial x_{i}}, i=1, \ldots, n$ formam uma base de $T_{p} M$.

Definição 1.0.5. Uma métrica riemanniana $g$ é uma aplicação que associa a cada ponto $p$ de $M$ um produto interno $g_{p}$, definido em $T_{p} M \times T_{p} M$ que varia suavemente com $p$.

Uma variedade diferenciável $(M, g)$ munida de uma métrica riemanniana é chamada variedade riemanniana. De fato, toda variedade suave admite métrica riemanniana; mais que isso, qualquer fibrado vetorial (não só $T M$ ) admite métrica definida nas fibras.

A aplicação $X_{p} \mapsto X_{p}(f)$ é linear e define um vetor cotangente $(\mathrm{d} f)_{p} \in T_{p}^{*} M$, por

$$
g_{p}(\mathrm{~d} f, X)=\left\langle(\mathrm{d} f)_{p}, X_{p}\right\rangle_{g}=X_{p}(f)
$$

com $X_{p} \in T_{p} M$. Analogamente, os diferenciais $d x^{i}$ das funções coordenadas, satisfazem

$$
\left\langle d x^{i}, \partial_{j}\right\rangle=\frac{\partial x_{i}}{\partial x_{j}}=\delta_{i j}
$$


O conjunto $\left\{d x^{i}\right\}$ é base dual a $\left\{\partial_{i}\right\}$ e, nesta base, o diferencial d $f$ tem coordenadas

$$
\left\langle\mathrm{d} f, \partial_{i}\right\rangle=\frac{\partial}{\partial x_{i}} \Longleftrightarrow \mathrm{d} f=\sum_{i} \frac{\partial f}{\partial x_{i}} d x^{i} .
$$

Um referencial $\left\{E_{i}\right\}$ em um aberto $U \subset M$ é uma coleção de $n$ campos vetoriais em $U$ que são linearmente independentes em cada $q \in U$ e, portanto, formam uma base de cada espaço tangente $T_{q} M$. Em cada $q \in U$ existe uma base dual $\left\{E_{q}^{i}\right\}$ em $T_{q}^{*}(M)$ e a coleção de aplicações $E^{i}: q \mapsto E_{q}^{i}$ é chamada referencial dual. Dado um sistema de coordenadas $\left(x_{1}, \ldots, x_{n}\right)$ em um aberto $U$, as derivadas parciais $\left\{\partial_{i}\right\}$ formam um referencial em $U$ e $\left\{d x^{i}\right\}$ é o referencial dual.

Definição 1.0.6. Seja $(M, g)$ uma variedade riemanniana orientada de dimensão $m$, a forma volume em $M$ é a única m-forma diferencial alternada, $\omega=\omega_{g}$, tal que, para cada $p \in M$, tem-se $\omega_{p}\left(e_{1}, \ldots, e_{m}\right)=1$, para toda base ortonormal (com respeito a $g_{p}$ ), positivamente orientada, do $T_{p} M$.

O volume de $M$ com respeito à métrica $g$ é dado por

$$
\mathcal{V}(g)=\int_{M} \omega_{g}
$$

como o volume de $M$ depende da métrica, é comum nos referirmos a ele simplesmente como o "volume da métrica $g$ ".

\subsection{Sistemas de Coordenadas em Variedades Riemannianas}

Em um sistema de coordenadas cartesianas $\left\{x_{i}\right\}_{i=1}^{m}$ em um espaço euclidiano, $\left\{\frac{\partial}{\partial x_{i}}\right\}_{i=1}^{m}$ é um referencial ortonormal para o espaço tangente com respeito à métrica euclidiana, portanto, a métrica riemanniana é dada pelo delta de Kronecker

$$
g_{i j}=\left\{\begin{array}{c}
1, i=j \\
0, i \neq j
\end{array} .\right.
$$

Sejam $(M, g)$ uma variedade riemanniana de dimensão $m$ e $p \in M$. Escolha um referencial ortonormal para o espaço tangente a $M$ em $p,\left\{e_{i}\right\}_{i=1}^{m}$ e defina o isomorfismo

$$
\varphi: \mathbb{R}^{m} \longrightarrow T_{p} M
$$

que leva a base canônica de $\mathbb{R}^{m}$ na base fixada para o espaço tangente. Considere a aplicação exponencial riemanniana baseada no ponto $p$,

$$
\exp _{p}: T_{p} M \longrightarrow M
$$

e seja $U$ uma vizinhança de $p$ em $M$ para a qual existe uma vizinhança $V$ da origem em $T_{p} M$ tal que $\left.\exp _{p}\right|_{V}: V \longrightarrow U$ é um difeomorfismo, isto é, para todo ponto $q \in U$ existe uma única geodésica ligando $p$ a $q$. Tal vizinhança $U$ é chamada vizinhança normal de $p$ e $(U, \psi)$, com

$$
\psi=\varphi^{-1} \circ \exp _{p}^{-1}: U \longrightarrow \mathbb{R}^{m},
$$

é uma carta local para $M$, chamada carta normal. Por outro lado, $\exp _{p} \circ \varphi$ define um sistema de coordenadas em $M$ chamado de sistema de coordenadas normais ou sistema de coordenadas geodésicas. Em um tal sistema são válidas as seguintes propriedades no ponto p:

i. $g_{i j}(p)=\delta_{i j}$, onde $\delta_{i j}$ é o delta de Kronecker, 
ii. a geodésica partindo de $p$ na direção de um vetor $X_{p}$ pode ser descrita por

$$
\gamma_{X}(t)=\left(t X^{1}, \ldots, t X^{m}\right),
$$

enquanto $\gamma$ estiver em $U$

iii. $\Gamma_{i j}^{k}(p)=0$, para todo $i, j, k \in\{1, \ldots, m\}$,

iv. $\partial_{k} g_{i j}(p)=0$, para todo $i, j, k \in\{1, \ldots, m\}$,

v. $\nabla_{i}=\partial_{i}$, em $p$, para todo $i=1, \ldots, m$.

Embora seja sempre possível construir um sistema de coordenadas normais em uma variedade riemanniana $M$, podemos garantir as boas propriedades que ele proporciona apenas no ponto $p$ e estas não podem ser estendidas para toda a variedade (a menos que $M$ seja flat). Contudo, existe um sistema de coordenadas locais que garante a validade de propriedades similares não apenas no ponto $p$, mas ao longo de uma geodésica partindo de $p$, numa vizinhança de $p$. Este sistema de coordenadas é chamado coordenadas Fermi em homenagem ao físico italiano Enrico Fermi, que primeiro as utilizou. Vejamos como podemos construir um tal sistema.

Seja $p$ um ponto em $M$ onde desejamos basear nosso sistema de coordenadas Fermi. Como fizemos antes, escolhemos um referencial ortonormal para o espaço tangente a $M$ em $p,\left\{e_{i}\right\}_{i=1}^{m}$ e consideramos a (única) geodésica $\gamma_{p}(t)$ partindo de $p$ na direção do vetor $\left(e_{1}\right)_{p}$, isto é,

$$
\gamma_{p}(0)=p \quad \text { e } \quad \gamma_{p}^{\prime}(0)=\left(e_{1}\right)_{p} .
$$

Agora, fazemos o transporte paralelo do referencial $\left\{e_{i}\right\}_{i=1}^{m}$ ao longo de $\gamma$, então $e_{1}(s)=\gamma_{p}^{\prime}(s)$ é o vetor tangente à geodésica no ponto $q=\gamma_{p}(s)$. Para $t$ pequeno, $(t, 0, \ldots, 0)$ representa, em coordenadas, a geodésica $\gamma_{p}(t)$, em uma vizinhança $W$ de $p$. Nesta vizinhança, ao longo de $\gamma, g$ é a métrica euclidiana e os símbolos de Christoffel se anulam. Consequentemente, as propriedades $(i$.$) a (v$.$) são satisfeitas ao longo de \gamma$, em $W$.

Esses sistemas de coordenadas especiais são úteis, pois facilitam as contas quando estamos interessados em informações locais.

\subsection{Variedades com Bordo}

O conceito de variedade com bordo é importante porque permite agregar ao conjunto de variedades diferenciáveis, espaços como a bola fechada, o toro sólido e a faixa de Möbius, além de permitir relacionar variedades de diferentes dimensões. Quando falamos em variedades com bordo, estamos falando de variedades definidas intrinsecamente, isto é, não como subconjuntos de um outro espaço topológico. Neste sentido, a ideia de bordo difere daquela de bordo de um conjunto.

Para definirmos uma variedade com bordo, $M$, de dimensão $m$, começamos escolhendo um modelo apropriado. Defina o semi-espaço real não-negativo, de dimensão $m$ por

$$
\mathbb{H}_{+}^{m}=\left\{\left(x_{1}, \cdots, x_{m}\right) \in \mathbb{R}^{m}: x_{m} \geq 0\right\}
$$

com a topologia induzida do $\mathbb{R}^{m}$, isto é, um subconjunto $U$ de $\mathbb{H}_{+}^{m}$ é aberto em $\mathbb{H}_{+}^{m}$ se $U=\mathbb{H}_{+}^{m} \cap W$, para algum subconjunto aberto $W$ de $\mathbb{R}^{m}$. O bordo do semi-espaço é

$$
\partial \mathbb{H}_{+}^{m}=\left\{\left(x_{1}, \cdots, x_{m}\right) \in \mathbb{R}^{m}: x_{m}=0\right\} .
$$

Definição 1.2.1. Uma variedade com bordo $M$, de dimensão $m$, é um espaço topológico Haussdorf, com base enumerável de abertos, tal que cada ponto $p \in M$ tem uma vizinhança $U \subset M$ homeomorfa a um aberto $V \subset \mathbb{H}_{+}^{m}$. 
Uma variedade diferenciável com bordo $M$, de dimensão $m$, é definida da mesma forma que no caso sem bordo, apenas observando que agora, temos dois tipos de cartas $\phi: U \longrightarrow V$ no atlas diferenciável: se $V \cap \partial \mathbb{H}_{+}^{m}=\emptyset$, temos uma carta interior, semelhante ao caso sem bordo; se $V \cap \partial \mathbb{H}_{+}^{m} \neq \emptyset$, dizemos que $\phi$ é uma carta de bordo. Um ponto $p$ é chamado ponto de bordo se existe uma carta $(\phi, U)$ tal que $\phi(p) \in \partial \mathbb{H}_{+}^{m}$ (se existe uma, qualquer outra, numa vizinhança de $p$, vai satisfazer a mesma condição), caso contrário, $p$ é um ponto interior.

O bordo da variedade $M$, denotado por $\partial M$ é uma $(m-1)$-variedade diferenciável sem bordo, $\partial(\partial M)=\emptyset$, que consiste de todos os pontos mapeados em $\left(x_{1}, \cdots, x_{m-1}, 0\right)$. O interior de $M$, é uma variedade diferenciável, sem bordo, de dimensão $m$.

É importante notar que, embora o produto cartesiano de variedades diferenciáveis seja uma variedade diferenciável, o produto cartesiano de duas variedades com bordo não é, em geral, uma variedade com bordo. Contudo, se $M$ é uma variedade diferenciável com bordo e $N$ é uma variedade difrenciável sem bordo, o produto cartesiano $M \times N$ é uma variedade com bordo, cujo bordo é dado por $\partial(M \times N)=\partial M \times N$.

Um importante resultado para variedades com bordo garante que $\partial M$ tem uma vizinhança difeomorfa ao produto $\partial M \times[0,1)$.

Teorema 1.2.2. Seja $M$ uma variedade diferenciável com bordo $\partial M$ compacto. Então existe uma vizinhança $V$ de $\partial M$ e um difeomorfismo $\Psi: \partial \times[0,1) \longrightarrow V$ tal que $\psi(x, 0)=x$ para todo $x \in \partial M$.

Demonstração. Considere uma cobertura finita $\left\{U_{i}\right\}$ do bordo $\partial M$, consistindo de abertos de $M$ tais que existam cartas locais $\varphi_{i}: W_{i} \longrightarrow \widetilde{W}_{i} \subset \mathbb{H}_{+}^{n}$ satisfazendo $U_{i} \subset \bar{U}_{i} \subset V_{i} \subset \bar{V}_{i} \subset W_{i} \operatorname{com} \bar{U}_{i}$ e $\bar{V}_{i}$ compactos. Seja $\widetilde{h}_{i}: \mathbb{H}_{+}^{n} \longrightarrow[0,1]$ uma função suave que vale 1 em $\widetilde{U}_{i}=\varphi_{i}\left(V_{i}\right)$ e 0 fora de $\widetilde{V}_{i}=\varphi_{i}\left(V_{i}\right)$. Seja $\widetilde{X}_{i}=h_{i} \frac{\partial}{\partial x_{n}}$, onde $\frac{\partial}{\partial x_{n}}$ é um campo de vetores em $\mathbb{H}^{n}$.

Defina $X_{i}=\varphi_{i}^{*}\left(\tilde{X}_{i}\right)$. Então, $X_{i}$ é um campo vetorial suave que se anula fora de um conjunto compacto e tal que para cada ponto $x \in \partial M$, temos $X_{i}(x)=0$ ou $X_{i}(x)$ é normal a $\partial M$ e aponta para o interior de $M$. Agora defina $X=\sum_{i} X_{i}$, daí $X$ é um campo suave que se anula fora de uma vizinhança compacta de $\partial M$ e para todo $x \in \partial M, X(x)$ é um campo normal a $\partial M$ apontando para o interior de $M$.

Portanto, existe $\varepsilon>0$ e uma aplicação suave $\psi: \partial M \times[0, \varepsilon) \longrightarrow M$ tal que $\psi(x, 0)=x$ e $t \longmapsto \psi(x, t)$ é uma curva integral de $X$. Tomando $\varepsilon$ suficientemente pequeno, $\psi$ é um difeomorfismo sobre uma vizinhança $V$ de $\partial M$ em $M$. Finalmente, defina $\Psi(x, t)=\psi(x, \varepsilon t)$.

\subsection{Tensores em Geometria Riemanniana ${ }^{1}$}

Seja $V$ um espaço vetorial real de dimensão $n<\infty$. Denotaremos por $V^{*}$ o espaço vetorial dual a $V$, isto é, o espaço de todos os funcionais lineares $V \longrightarrow \mathbb{R}$. Escreveremos $\left\langle v^{*}, v\right\rangle$ para denotar $v^{*}(v)$, onde $v^{*} \in V^{*}$ e $v \in V$.

Lembre que $V$ e $V^{*}$ são isomorfos, mas esse isomorfismo não é, em geral, canônico, ou seja, depende da base escolhida. Seja $\left\{e_{1}, \ldots, e_{n}\right\}$ uma base de $V$, então todo elemento $v$ de $V$ é escrito de maneira única como $\sum_{i=1}^{n} \alpha^{i} e_{i}$, para escalares $\alpha^{i} \in \mathbb{R}, i=1, \ldots, n$. Defina $e^{i}$ por

$$
e^{i}\left(\sum_{i=1}^{n} \alpha^{i} e_{i}\right)=\alpha^{i},
$$

$\left\{e^{1}, \ldots, e^{n}\right\}$ é uma base para $V^{*}$, chamada de base dual de $\left\{e_{1}, \ldots, e_{n}\right\}$. Para a base dual, temos

$$
\left\langle e^{i}, e_{j}\right\rangle=\delta_{i j}
$$

\footnotetext{
${ }^{1}$ Esta Seção é baseada em notas de aula do professor Svante Janson [38], da Universidade de Uppsala, Suécia, disponível em: <http://www2.math.uu.se/ svante/papers/>.
} 
onde $\delta_{i j}$ é o Delta de Kronecker. Já entre $V$ e $V^{* *}$, o chamado espaço bidual, existe isomorfismo canônico

$$
\begin{gathered}
V \ni v \longleftrightarrow v^{* *} \in V^{* *}, \\
\left\langle v^{* *}, v^{*}\right\rangle=\left\langle v^{*}, v\right\rangle,
\end{gathered}
$$

e $\left\{e_{1}, \ldots, e_{n}\right\}$ é uma base de $V^{* *}$, dual à base $\left\{e^{1}, \ldots, e^{n}\right\}$.

Seja $\left\{f_{1}, \ldots, f_{n}\right\}$ outra base de $V$, então

$$
f_{i}=\sum_{j} a_{i j} e_{j}
$$

e $\left\{f^{1}, \ldots, f^{n}\right\}$ a correspondente base dual (outra base $V^{*}$ ), com

$$
f^{i}=\sum_{j} b_{i j} e^{j}
$$

Assim, temos

$$
\delta_{i j}=\left\langle f^{i}, f_{j}\right\rangle=\sum_{k} b_{i k} e^{k}\left(\sum_{\ell} a_{j \ell} e_{\ell}\right)=\sum_{k, \ell} b_{i k} a_{j \ell} e^{k}\left(e_{\ell}\right)=\sum_{k} b_{i k} a_{j k},
$$

isto é, por um lado

$$
\left(\left\langle f^{i}, f_{j}\right\rangle\right)_{i j}=I
$$

onde $I$ é a matriz identidade, por outro lado,

$$
\left(\left\langle f^{i}, f_{j}\right\rangle\right)_{i j}=B A^{t}
$$

$\operatorname{com} A=\left(a_{i j}\right)_{i j}$ e $B=\left(b_{i j}\right)_{i j}$. Logo, $B^{-1}=A^{t}$ ou $B=\left(A^{t}\right)^{-1}=\left(A^{-1}\right)^{t}$.

Definição 1.3.1. Dados $k$, $\ell$, inteiros não negativos, $\mathcal{J}^{k, \ell}(V)$ é o espaço vetorial de todas as aplicações multilineares

$$
\begin{aligned}
T: V^{*} \times \ldots \times V^{*} \times V \times \ldots \times V & \longrightarrow \mathbb{R} \\
\left(v_{1}^{*}, \ldots, v_{k}^{*}, v_{1}, \ldots, v_{\ell}\right) & \longmapsto T\left(v_{1}^{*}, \ldots, v_{k}^{*}, v_{1}, \ldots, v_{\ell}\right)
\end{aligned}
$$

com $k$ variáveis em $V^{*}$ e $\ell$ variáveis em $V$. Os elementos de $\mathcal{J}^{k, \ell}(V)$ são chamados tensores de grau (ou ordem) $(k, \ell)$, ou simplesmente, $(k, \ell)$-tensores.

Se $\left\{e_{1}, \ldots, e_{n}\right\}$ é uma base de $V$ e $T \in \mathcal{J}^{k, \ell}(V)$, os $n^{k+\ell}$ números

$$
T_{j_{1} \ldots j_{\ell}}^{i_{1} \ldots i_{k}}=T\left(e^{i_{1}}, \ldots, e^{i_{k}}, e_{j_{1}}, \ldots, e_{j_{\ell}}\right)
$$

são os coeficientes do tensor $T$ e $T$ fica completamente determinado por eles. Assim, o espaço $\mathcal{J}^{k, \ell}(V)$ tem dimensão $n^{k+\ell}$.

Tensores do tipo $(k, 0)$, isto é, agem apenas em elementos do espaço dual, são chamados contravariantes e é conveniente sobrescrevermos os índices dos coeficientes nesse caso. Já os tensores do tipo $(0, \ell)$ são chamados covariantes e utilizamos índices subscritos para eles.

Exemplo 1.3.2. Observe que $\mathcal{J}^{0,0}(V)=\mathbb{R}, \mathcal{J}^{0,1}(V)=V^{*}$ e $\mathcal{J}^{1,0}(V)=V^{* *} \simeq V$.

Observação 1.3.3. Note que uma aplicação multilinear $S: V^{\ell} \longrightarrow V$ define um $(1, \ell)$-tensor da seguinte forma

$$
T\left(v^{*}, v_{1}, \ldots, v_{\ell}\right)=\left\langle v^{*}, S\left(v_{1}, \ldots, v_{\ell}\right)\right\rangle,
$$

dai existe uma identificação entre $\mathcal{J}^{1, \ell}(V)$ e o espaço dessas aplicações multilineares, denotado por 
$\operatorname{Hom}(\underbrace{V, \ldots, V}_{\ell \text { vezes }} ; V)$.

\section{Produto Tensorial}

Dois tensores de mesmo grau podem ser somados, pois $\mathcal{J}^{k, \ell}(V)$ é um espaço vetorial. Além disso, podemos definir um produto entre tensores. Sejam $T$ um $(k, \ell)$-tensor e $S$ um $(r, s)$-tensor, então o $(k+r, \ell+s)$-tensor

$$
T \otimes S\left(v_{1}^{*}, \ldots, v_{k+r}^{*}, v_{1}, \ldots, v_{\ell+s}\right)=T\left(v_{1}^{*}, \ldots, v_{k}^{*}, v_{1}, \ldots, v_{\ell}\right) \cdot S\left(v_{k+1}^{*}, \ldots, v_{k+r}^{*}, v_{\ell+1}, \ldots, v_{\ell+s}\right) .
$$

Os coeficientes de $T \otimes S$ são dados pelo produto dos coeficientes de $T$ e de $S$. O produto tensorial é associativo e distributivo com respeito à soma, mas não é, em geral, comutativo.

Agora, se $\left\{e_{1}, \ldots, e_{n}\right\}$ é uma base de $V$. Para índices $i_{1}, \ldots, i_{k}, j_{1}, \ldots, j_{\ell} \in\{1,2, \ldots, n\}$ podemos formar o produto

$$
e_{i_{1}} \otimes \ldots \otimes e_{i_{k}} \otimes e^{j_{1}}, \otimes \ldots \otimes e^{j_{\ell}} \in \mathcal{J}^{k, \ell}(V)
$$

onde $e_{i} \in \mathcal{J}^{1,0}(V), e^{j} \in \mathcal{J}^{0,1}(V)$, cujos coeficientes são dados por

$$
e_{i_{1}} \otimes \ldots \otimes e_{i_{k}} \otimes e^{j_{1}}, \otimes \ldots \otimes e^{j_{\ell}}\left(e^{i_{1}^{\prime}}, \ldots, e^{i_{k}^{\prime}}, e_{j_{1}^{\prime}}, \ldots, e_{j_{\ell}^{\prime}}\right)=\delta_{i_{1} i_{1}{ }_{1} \delta_{i_{2} i_{2}{ }_{2}} \ldots \delta_{j_{\ell} j_{\ell}{ }_{\ell}}}
$$

na base $\left\{e_{1}, \ldots, e_{n}\right\}$, isto é, exatamente um coeficiente é igual a 1 e os demais são nulos. Portanto, o conjunto de $n^{k+\ell}$ tensores $\left\{e_{i_{1}} \otimes \ldots \otimes e_{i_{k}} \otimes e^{j_{1}}, \otimes \ldots \otimes e^{j_{\ell}}\right\}$ forma uma base de $\mathcal{J}^{k, \ell}(V)$ e os coeficientes (1.1) do tensor $T \in \mathcal{J}^{k, \ell}(V)$ são as coordenadas de $T$ nesta base:

$$
T=\sum_{i_{1}, \ldots, i_{k}, j_{1}, \ldots, j_{\ell}} T_{j_{1} \ldots j_{\ell}}^{i_{1} \ldots i_{k}} e_{i_{1}} \otimes \ldots \otimes e_{i_{k}} \otimes e^{j_{1}}, \otimes \ldots \otimes e^{j_{\ell}} .
$$

\section{Contração}

Um (1,1)-tensor $T$ pode ser visto como um operador linear $S: V \longrightarrow V$, fazendo

$$
T\left(v^{*}, v\right)=\left\langle v^{*}, S(v)\right\rangle .
$$

O traço desse operador é chamado contração do $(1,1)$-tensor $T$. Dada $\left\{e_{i}\right\}$ uma base ortonormal de $V$ e $\left\{e^{j}\right\}$ a base dual, a contração de $T$ é dada por

$$
\sum_{i}\left\langle e^{i}, S\left(e_{i}\right)\right\rangle=\sum_{i} T\left(e^{i}, e_{i}\right)=\sum_{i} T_{i}^{i}
$$

onde $\left\langle e^{i}, S\left(e_{i}\right)\right\rangle=e^{i}\left(S\left(e_{i}\right)\right)$ é a $i$-ésima coordenada do vetor $S\left(e_{i}\right)$ na base $\left\{e_{i}\right\}$, isto é, a entrada ii da matriz do operador $S$,

$$
a_{i j}=e^{i}\left(S\left(e_{j}\right)\right)
$$

Para $T$ um $(k, \ell)$-tensor, escolha um argumento covariante e um contravariante, mantenha os demais fixos, então

$$
T: V^{*} \times V \longrightarrow \mathbb{R}
$$

torna-se uma aplicação bilinear, isto é, um $(1,1)$-tensor para o qual, agora, temos contração como definida acima. Esta contração é um número real que depende linearmente dos outros $(k-1)+(\ell-1)$ argumentos e, portanto, define um tensor de grau $(k-1, \ell-1)$ ao qual chamamos contração de T.

Exemplo 1.3.4. O $(1,2)$-tensor $\widetilde{T}$ cujas coordenadas na base canônica são dadas por

$$
\widetilde{T}_{j k}^{i}=\sum_{m} T_{m j k}^{i m}
$$


é a contração de um (2,3)-tensor $T$.

\section{Produto Exterior}

Seja $\Lambda_{k}(V)$ o espaço vetorial dos $k$-tensores covariantes ( $(0, k)$-tensores $)$ antissimétricos, com $\operatorname{dim} \Lambda_{k}(V)=\left(\begin{array}{l}n \\ k\end{array}\right), n>k$. Se $n<k, \operatorname{dim} \Lambda_{k}(V)=0$ e portanto $\Lambda_{k}(V)=\{0\}$. Além disso, $\operatorname{dim} \Lambda_{n}(V)=1, \Lambda_{0}(V)=\mathbb{R}$ e $\Lambda_{1}(V)=V^{*}$. Podemos escrever a soma direta, $\Lambda(V)=$ $\bigoplus_{k=0}^{\infty} \Lambda_{k}(V)=\bigoplus_{k=0}^{n} \Lambda_{k}(V)$ com dimensão $\sum_{k=0}^{n}\left(\begin{array}{l}n \\ k\end{array}\right)=2^{n}$.

Seja $T \in \Lambda_{k}(V), S \in \Lambda_{\ell}(V), k, \ell \geq 0$. O produto exterior de $T$ e $S$ é o tensor $T \wedge S \in \Lambda_{k+\ell}(V)$ definido por

$$
T \wedge S\left(v_{1}, \ldots, v_{k+\ell}\right)=\frac{1}{k ! \ell !} \sum_{\sigma \in S_{k+\ell}} \operatorname{sgn}(\sigma) T\left(v_{\sigma(1)}, \ldots, v_{\sigma(k)}\right) S\left(v_{\sigma(k+1)}, \ldots, v_{\sigma(k+\ell)}\right) .
$$

O produto exterior é bilinear, associativo e antissimétrico, isto é, satisfaz $T \wedge S=(-1)^{k \ell} T \wedge S$. Se $v_{1}^{*}, \ldots, v_{k}^{*} \in V^{*}=\Lambda_{1}(V)$, então $v_{1}^{*} \wedge \ldots \wedge v_{k}^{*} \in \Lambda_{k}(V)$ é o produto exterior de 1 -tensores alternados, dado por

$$
v_{1}^{*} \wedge \ldots \wedge v_{k}^{*}\left(v_{1}, \ldots, v_{k}\right)=\operatorname{det}\left(\left\langle v_{i}^{*}, v_{j}\right\rangle\right)_{i, j=1}^{k} .
$$

Dada $\left\{e_{1}, \ldots, e_{n}\right\}$ base de $V$ e $\left\{e^{1}, \ldots, e^{n}\right\}$ base dual,

$$
\left\{e^{i_{1}} \wedge \ldots \wedge e^{i_{k}}, 1 \leq i_{1}<\ldots<i_{k} \leq n\right\}
$$

é base de $\Lambda_{k}(V)$ e

$$
T=\sum_{1 \leq i_{1}<\ldots<i_{k} \leq n} T_{i_{1}, \ldots, i_{k}} e^{i_{1}} \wedge \ldots \wedge e^{i_{k}}
$$

\section{Tensores sobre um Espaço Euclidiano}

Seja $V$ um espaço euclidiano com produto interno denotado por $g$ ou por $\langle,\rangle_{g}$. Então, existe um isomorfismo natural entre $V$ e seu dual, dado por

$$
\begin{aligned}
& \phi \quad: \quad \longrightarrow V^{*} \\
& v \longmapsto h(v): V \longrightarrow \mathbb{R} \\
& w \longmapsto\langle v, w\rangle_{g} .
\end{aligned}
$$

Assim, $g$ induz um produto interno em $V^{*}$ dado por $g^{*}\left(v^{*}, w^{*}\right)=g^{*}(h(v), h(w))=g(v, w)$. Note que $g^{*}$ é uma forma bilinear e portanto um $(2,0)$-tensor contravariante. Lembre que usamos $\left\langle v^{*}, w\right\rangle$ também para denotar $v^{*}(w)$, mas isso não é um problema já que as notações coincidem:

$$
\langle h(v), w\rangle=h(v)(w)=\langle v, w\rangle_{g}=\langle h(v), h(w)\rangle_{g^{*}}=\left\langle v^{*}, w^{*}\right\rangle_{g^{*}} .
$$

Seja $\left\{e_{i}\right\}_{i=1}^{n}$ uma base de $V$ e sejam $g_{i j}$ e $g^{i j}$ as coordenadas dos produtos internos de $V$ e $V^{*}$, respectivamente. Se $v^{*} \in V^{*}$, temos

$$
\begin{aligned}
v_{i}^{*} & =v^{*}\left(e_{i}\right)=\left\langle v^{*}, e_{i}\right\rangle=\left\langle v, e_{i}\right\rangle_{g}=\left\langle\sum_{j} v^{j} e_{j}, e_{i}\right\rangle_{g}=\sum_{j} v^{j}\left\langle e_{j}, e_{i}\right\rangle_{g} \\
& =\sum_{j} v^{j} g\left(e_{j}, e_{i}\right)=\sum_{j} v^{j} g_{j i}=\sum_{j} g_{i j} v^{j},
\end{aligned}
$$


isto é, obtemos as coordenadas de $v^{*}$ em termos das coordenadas de $v$, fazendo

$$
\left(\begin{array}{c}
v_{1}^{*} \\
v_{2}^{*} \\
\vdots \\
v_{n}^{*}
\end{array}\right)=\left(\begin{array}{cccc}
g_{11} & g_{12} & \ldots & g_{1 n} \\
g_{21} & g_{22} & \ldots & g_{2 n} \\
\vdots & \vdots & \ddots & \vdots \\
g_{n 1} & g_{n 2} & \ldots & g_{n n}
\end{array}\right)\left(\begin{array}{c}
v^{1} \\
v^{2} \\
\vdots \\
v^{n}
\end{array}\right)
$$

Analogamente, temos $v^{i}=\sum_{j} g^{i j} v_{j}^{*}$.

Observe que

$$
v^{i}=\sum_{j} g^{i j} v_{j}^{*} \Longrightarrow \sum_{i} g_{i j} v^{i}=\sum_{i} g_{i j}\left(\sum_{j} g^{i j} v_{j}^{*}\right)=\sum_{i j} g_{i j} g^{i j} v_{j}^{*},
$$

por outro lado,

$$
\sum_{i} g_{i j} v^{i}=v_{j}^{*}
$$

portanto, a matriz de $g^{*},\left(g^{i j}\right)_{i j}$ é a inversa de $\left(g_{i j}\right)_{i j}$, que é a matriz de $g$.

Por esta razão, ao multiplicarmos as coordenadas de $v$ pelas entradas da matriz de $g$, dizemos que estamos abaixando o índice e ao multiplicarmos as coordenadas de $v^{*}$ pelas entradas da matriz de $g^{-1}$, dizemos que estamos levantando o índice.

A convenção que estamos utilizando para os índices dos vetores covariantes e contravariantes põe em posições opostas os índices com respeito aos quais estamos somando, o que elimina a necessidade do símbolo de somatório. Por exemplo, podemos escrever apenas $g^{i j} v_{j}^{*}$ para denotar $\sum_{j} g^{i j} v_{j}^{*}$; esta é a chamada notação de Einstein.

O isomorfismo (1.2) entre $V=\mathcal{J}^{1,0}(V)$ e $V^{*}=\mathcal{J}^{0,1}$ se estende a tensores de graus mais altos. Assim, um tensor de grau $(k, l)$, que é uma aplicação multilinear definida em $V^{* k} \times V^{\ell}$, pode ser identificado com um $(0, k+\ell)$-tensor em $V^{k+\ell}$, isto é, $\mathcal{J}^{k, \ell}(V) \simeq \mathcal{J}^{0, k+\ell}(V)$, de modo que podemos considerar apenas tensores covariantes, ou contravariantes, conforme for conveniente.

Exemplo 1.3.5. Seja $T$ um $(0,2)$-tensor de coordenadas $T_{i j}$, então $T$ corresponde a

- um (1,1)-tensor cujas coordenadas são $T_{j}^{i}=g^{i k} T_{k j}$, ou

- um $(1,1)$-tensor cujas coordenadas são $T_{i}{ }^{j}=g^{j k} T_{i k}$, ou

- um (2,0)-tensor contravariante, $T^{i j}=g^{i k} g^{j \ell} T_{k \ell}$.

Observe que se $\left\{e_{i}\right\}$ é uma base ortonormal de $V$, então a base dual $\left\{e^{i}\right\}$ é uma base ortonormal de $V^{*}$ e temos $g_{i j}=\delta_{i j}$ e $g^{i j}=\delta^{i j}$, onde $\delta$ é o delta de Kronecker. Neste caso, a menos das posições dos índices, os coeficientes do $(0,2)$-tensor do exemplo acima, continuam sendo os mesmos: $T^{i j}=T_{j}^{i}=T_{i}{ }^{j}=T_{i j}$.

\section{Tensores em Variedades}

Seja $M$ uma variedade diferenciável. Para cada $p \in M$, seja $\mathcal{J}_{p}^{k, \ell}(M)$ o espaço dos $(k, \ell)$ tensores sobre $T_{p} M$. Em particular, um tensor em $p$ de grau $(1,0)$ é um vetor tangente, em $T_{p} M$ e um tensor em $p$ de grau $(0,1)$ é um vetor cotangente, em $T_{p}^{*} M$.

Sejam $\left\{E_{i}\right\}$ um referencial em uma vizinhança $U$ de $p \in M$ e $\left\{E^{i}\right\}$ o referencial dual. Um campo tensorial $T$ de grau $(k, \ell)$ em $U$ fica determinado por suas coordenadas com respeito a estes referenciais, isto é,

$$
T_{j_{1}, \ldots, j_{\ell}}^{i_{1}, \ldots, i_{k}}(q)=T\left(E^{i_{1}}, \ldots, E^{i_{k}}, E_{j_{1}}, \ldots, E_{j_{\ell}}\right)(q),
$$

que são funções em $U$. Dizemos que $T$ é um campo tensorial diferenciável em $U$, se cada uma das funções coordenadas é $\mathcal{C}^{\infty}$ em $U$. A união disjunta dos espaços dos $(k, \ell)$-tensores sobre $T_{p} M$ para todo $p$ é chamada fibrado dos $(k, \ell)$-tensores em $M$, denotado por $\mathcal{J}^{k, \ell}(M)$. 
Exemplo 1.3.6. Uma métrica riemanniana $g$ em uma variedade diferenciável $M$ pode ser vista como uma seção suave do fibrado dos (0,2)-tensores simétricos positivo definidos em $T M$,

$$
g: M \longrightarrow T^{*} M \otimes T^{*} M,
$$

ou como uma aplicação $\mathcal{C}^{\infty}$-bilinear ${ }^{2}$

$$
g: \mathfrak{X}(M) \times \mathfrak{X}(M) \longrightarrow \mathcal{C}^{\infty}(M),
$$

com $g(X, X)>0, \forall X \neq 0$. Isto é, $g_{p} \in T_{p}^{*} M \otimes T_{p}^{*} M$ é um (0,2)-tensor simétrico positivo definido. Em coordenadas locais $x_{1}, \ldots, x_{m}$, podemos escrever

$$
g=\sum_{i, j} g_{i j} d x^{i} \otimes d x^{j}
$$

onde $g_{i j}=g\left(\frac{\partial}{\partial x_{i}}, \frac{\partial}{\partial x_{j}}\right)$ são funções suaves para cada $p \in M$, chamadas coordenadas do tensor métrico; $g_{i j}$ são as entradas da matriz do tensor métrico:

$$
g_{p}(X, Y)=[X]^{t}\left(g_{i j}\right)_{i, j}[Y]
$$

onde $[X],[Y]$ são as coordenadas dos vetores $X_{p}$ e $Y_{p}$ com respeito à base $\left\{\frac{\partial}{\partial x_{i}}\right\}_{j=1}^{m}$ do $T_{p} M$. Denote por $g^{i j}$ as entradas da matriz inversa do tensor métrico. Uma métrica riemanniana $g$ em uma variedade $M$ induz produtos internos e normas em todos os espaços de tensores definidos em $M$. Dada uma métrica $g$, a identificação

$$
T M \ni X \longmapsto g(X, \cdot) \in T^{*} M,
$$

entre $T M$ e $T^{*} M$, define uma métrica em $T^{*} M$. De fato, se $x_{1}, \ldots, x_{m}$ são coordenadas locais em $M$, temos

$$
\frac{\partial}{\partial x_{i}} \longmapsto g_{i j} d x^{j} \quad \text { e } \quad d x^{i} \longmapsto g^{i j} \frac{\partial}{\partial x_{j}}
$$

já que,

$$
\frac{\partial}{\partial x_{i}} \longmapsto g\left(\frac{\partial}{\partial x_{i}}, Y\right)=Y_{i}=g_{i j} Y^{j}=g_{i j} d x^{j}(Y),
$$

para todo $Y \in \mathfrak{X}(M)$. A componente $i j$ da métrica $g^{*}$ induzida em $T^{*} M$ é dada por

$$
g_{i j}^{*}=g^{*}\left(d x^{i}, d x^{j}\right)=g\left(g^{i k} \frac{\partial}{\partial x_{k}}, g^{j l} \frac{\partial}{\partial x_{l}}\right)=g^{i k} g^{j l} g_{k l}=g^{i k} g_{k}^{j}=g^{i j} .
$$

Exemplo 1.3.7. O colchete de Lie $(X, Y) \mapsto[X, Y]$ para campos suaves em $M$ é bilinear, mas não é $\mathcal{C}^{\infty}$-bilinear, portanto não é um tensor.

Exemplo 1.3.8. Uma conexão afim em uma variedade riemanniana $M$ não é $\mathcal{C}^{\infty}$-bilinear, portanto não é um tensor, por outro lado, se fixarmos $Y$, então $X \mapsto \nabla_{X} Y$ é $\mathcal{C}^{\infty}$-bilinear de $\mathfrak{X}(M) \longrightarrow \mathfrak{X}(M)$ e pode ser vista como um $(1,1)$-tensor.

Em uma variedade diferenciável $M$ sempre podemos derivar funções suaves com respeito a um campo vetorial $X$. Dada uma conexão afim $\nabla$, podemos, também, derivar campos vetoriais em $M$ (derivada covariante). Essa conexão $\nabla$ induz uma conexão afim nos fibrados tensoriais de $M$.

\footnotetext{
${ }^{2}$ Com $\mathcal{C}^{\infty}$-bilinear queremos dizer que $g$ satisfaz $g(f X, Y)=g(X, f Y)=f g(X, Y), \forall f \in \mathcal{C}^{\infty}(M)$.
} 
Para um $(k, \ell)$-campo tensorial, a conexão induzida, que também denotaremos por $\nabla$, é dada por

$$
\begin{aligned}
\left(\nabla_{X} T\right)\left(\omega_{1}, \ldots, \omega_{k}, Y_{1}, \ldots, Y_{\ell}\right) & =\nabla_{X}\left(T\left(\omega_{1}, \ldots, \omega_{k}, Y_{1}, \ldots, Y_{\ell}\right)\right) \\
& \left.\left.-T\left(\nabla_{X} \omega_{1}, \ldots, \omega_{k}, Y_{1}, \ldots, Y_{\ell}\right)\right)-\ldots-T\left(\omega_{1}, \ldots, \nabla_{X} \omega_{k}, Y_{1}, \ldots, Y_{\ell}\right)\right) \\
& \left.\left.-T\left(\omega_{1}, \ldots, \omega_{k}, \nabla_{X} Y_{1}, \ldots, Y_{\ell}\right)\right)-\ldots-T\left(\omega_{1}, \ldots, \omega_{k}, Y_{1}, \ldots, \nabla_{X} Y_{\ell}\right)\right)
\end{aligned}
$$

para quaisquer 1 -formas $\omega_{i}, i=1, \ldots, k$ e campos vetoriais $Y_{j}, j=1, \ldots, \ell$.

Exemplo 1.3.9. Seja $M$ uma variedade riemanniana com métrica $g$ e conexão afim $\nabla$. Sejam $X, Y, Z$ campos vetoriais em $M$, então

$$
\left(\nabla_{X} g\right)(Y, Z)=X(g(Y, Z))-g\left(\nabla_{X} Y, Z\right)-g\left(Y, \nabla_{X} Z\right)
$$

Se $\nabla$ é a conexão Levi-Civita, a condição $X(g(Y, Z))=g\left(\nabla_{X} Y, Z\right)+g\left(Y, \nabla_{X} Z\right)$ equivale a $\nabla g=0$.

\section{Tensores em Variedades Riemannianas}

Seja $(M, g)$ uma variedade riemanniana com conexão Levi-Civita $\nabla$. Note que a métrica riemanniana faz de cada espaço tangente $T_{p} M$ um espaço euclidiano. O tensor curvatura de Riemann, $R$ tem uma forma covariante, que é um $(0,4)$-tensor, que se relaciona com o $(1,3)$-tensor por

$$
R(X, Y, Z, W)=\langle R(X, Y) Z, W\rangle
$$

para campos vetoriais $X, Y, Z, W$. Sejam $g_{i j}$ e $g^{i j}$ as coordenadas do tensor métrico e seu inverso, respectivamente, com respeito a uma carta local. Sejam $R_{i j k}^{\ell}$ as coordenadas do (1,3)-tensor de Riemann e $R_{i j k \ell}$ as coordenadas do $(0,4)$-tensor de Riemann, note que elas são obtidas uma da outra ao levantarmos ou abaixarmos o índice

$$
\begin{gathered}
R_{i j k}^{\ell}=g^{\ell m} R_{i j k m}, \\
R_{i j k \ell}=g_{\ell m} R_{i j k}^{m} .
\end{gathered}
$$

O tensor de Ricci é o obtido pela contração do tensor de Riemann, assim, se $R_{i j}$ são as coordenadas do tensor de Ricci, temos

$$
R_{i j}=R_{i k j}^{k}=g^{k \ell} R_{i k j \ell}=g^{k \ell} R_{k i \ell j},
$$

além disso, a curvatura escalar $R$ é obtida da contração do tensor de Ricci

$$
R=g^{i j} R_{i j}=g^{i k} g^{j \ell} R_{i j k \ell}
$$

Observe que, se $X$ é um campo vetorial e $T$ é um campo tensorial em $M$, então

$$
\nabla_{X}(g \otimes T)=\left(\nabla_{X} g\right) \otimes T+g \otimes \nabla_{X} T=g \otimes \nabla_{X} T
$$

assim, $\nabla_{X}$ comuta com contrações e levantamentos/abaixamentos de índices.

\subsection{Curvaturas}

Nesta seção, gostaríamos de dar algumas noções intuitivas e interpretações geométricas de curvaturas em variedades riemannianas.

Definição 1.4.1. Seja $(E, M, \pi)$ um fibrado vetorial $e \Gamma(E)$ o conjunto das seções de $E$, isto é, o conjunto das aplicações $s: M \rightarrow E$ tais que $\pi \circ s=i d$. Uma conexão afim é uma aplicação bilinear

$$
\nabla: \mathfrak{X}(M) \times \Gamma(E) \longrightarrow \Gamma(E)
$$


que satisfaz

- $\nabla_{f X} Y=f \nabla_{X} Y$,

- $\nabla_{(X+Y)} Z=\nabla_{X} Z+\nabla_{Y} Z$

- $\nabla_{X}(f Y)=f \nabla_{X} Y+(X \cdot f) Y$,

- $\nabla_{X}(Y+Z)=\nabla_{X} Y+\nabla_{X} Z$,

onde $X \in \mathfrak{X}(M), Y, Z \in \Gamma(E)$ e $f \in \mathcal{C}^{\infty}(M)$.

Uma conexão $\nabla$ em $T M$ induz uma conexão $\nabla^{*}$ em $T^{*} M$ : sejam $Y \in \Gamma(T M)=\mathfrak{X}(M)$ e $\omega \in \Gamma\left(T^{*} M\right)$, isto é, para cada $p \in M$, temos

$$
p \longmapsto Y_{p} \in T_{p} M, \quad \text { e } \quad p \longmapsto \omega_{p} \in T_{p}^{*} M .
$$

Como $\omega_{p}: T_{p} M \longrightarrow \mathbb{R}$ associa a cada vetor tangente a $M$ em $p$ um número real $\omega_{p}\left(Y_{p}\right)$, para cada campo diferenciável $Y$ em $M$ temos que $\omega(Y)$ é uma função suave: $p \longmapsto \omega_{p}\left(Y_{p}\right)$. Qualquer que seja $X \in \mathfrak{X}(M)$ temos

$$
X \cdot \omega(Y)=\left(\nabla_{X}^{*} \omega\right) Y+\omega\left(\nabla_{X} Y\right)
$$

portanto, $\nabla^{*}$ fica definida por

$$
\left(\nabla_{X}^{*} \omega\right) Y=X \cdot \omega(Y)-\omega\left(\nabla_{X} Y\right)
$$

Em particular, para todo $Y, W \in \Gamma(T M)$, temos que $g(Y, W)$ é o traço de uma seção de $T^{*} M \otimes$ $T^{*} M \otimes T M \otimes T M$ e, para qualquer campo $X \in \mathfrak{X}(M)$, temos

$$
X \cdot g(Y, W)=\left(\nabla_{X}^{*} g\right)(X, Y)+g\left(\nabla_{X} Y, W\right)+g\left(Y, \nabla_{X} W\right) .
$$

Definição 1.4.2. Seja $(M, g)$ uma variedade riemanniana de dimensão $m \geq 2$. Uma conexão afim em $T M$ é chamada conexão riemanniana, ou conexão Levi-Civita, se, para todo $X, Y, Z \in \mathfrak{X}(M)$, satisfaz

- $X \cdot g(Y, Z)=g\left(\nabla_{X} Y, Z\right)+g\left(Y, \nabla_{X} Z\right)$,

- $\nabla_{X} Y-\nabla_{Y} X=[X, Y]$.

Em particular, se $\nabla$ é uma conexão riemanniana, temos $\left(\nabla_{X}^{*} g\right)=0$.

Dada uma variedade riemanniana $M$, existe uma única conexão riemanniana em $T M$, determinada pela fórmula de Koszul:

$$
\begin{aligned}
2 g\left(\nabla_{X} Y, Z\right) & =X g(Y, Z)+Y g(Z, X)-Z g(X, Y) \\
& -g([X, Y], Z)-g([Y, Z], X)+g([Z, X], Y)
\end{aligned}
$$

A conexão riemanniana permite o cálculo de curvaturas em variedades riemannianas $(M, g)$, tais curvaturas são grandezas que dependem da métrica $g$.

A curvatura gaussiana de uma superfície $S$, de dimensão 2 , imersa em $\mathbb{R}^{3}$, é dada por

$$
K:=\kappa_{1} \cdot \kappa_{2}
$$

onde $\kappa_{1}$ e $\kappa_{2}$ são as curvaturas principais. A curvatura $K$ será positiva, se $\kappa_{1}$ e $\kappa_{2}$ tiverem o mesmo sinal (ambas positivas ou ambas negativas); $K$ será nula se $\kappa_{1}=0$ ou $\kappa_{2}=0$; e $K$ será negativa se $\kappa_{1}$ e $\kappa_{2}$ têm sinais opostos.

Seja $p \in M$. Lembre que $\kappa_{1}(p)$ e $\kappa_{2}(p)$ indicam a curvatura normal máxima e a mínima, respectivamente, das curvas passando por $p$ (sabemos que todas as curvas que passam por $p$ e na direção $V$ têm a mesma curvatura normal). 
Geometricamente, se $K(p)>0$, as curvas de curvatura extrema que passam por $p$ curvam na mesma direção normal (veja curvas vermelha e verde no toro abaixo), a superfície $S$ no ponto $p$ se curva como um paraboloide elíptico (uma cuia) e o ponto $p$ é, por isso, chamado de ponto elíptico.

Se $K(p)=0$, pelo menos uma das curvas de curvatura extrema que passam por $p$ tem curvatura zero na direção normal à $S$ (como a curva amarela no toro), em algum sentido a superfície se curva como um cilindro parabólico (uma folha de papel) e daí $p$ é dito um ponto parabólico.

Se $K(p)<0$, as curvas de curvatura máxima que passam por $p$ curvam-se, na direção normal a $S$, em sentidos opostos (como as curvas azul e verde da figura), nesse caso, a superfície se curva como o paraboloide hiperbólico no ponto $p$ e ele é dito um ponto sela.

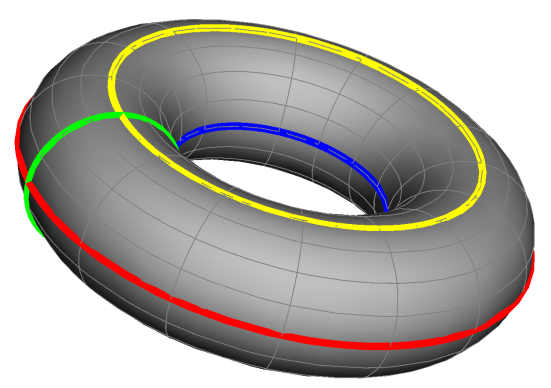

Figura 1.1:

A curvatura média de uma superfície $S$ em um ponto $p$ é a média das curvaturas principais

$$
H(p)=\frac{\kappa_{1}+\kappa_{2}}{2}
$$

As curvaturas gaussiana e média são ambas definidas em termos de curvaturas que têm por referência a direção normal à superfície $S$, o que pressupõe que a superfície (de dimensão 2) está mergulhada em um "ambiente maior" (de dimensão 3). Apesar disso, Gauss provou, em seu Notável Teorema, que a curvatura gaussiana não depende do ambiente onde a superfície está inserida, por assim dizer. A curvatura gaussiana é uma medida intrínseca à superfície, depende apenas da estrutura riemanniana de $S$. A curvatura média é uma medida extrínseca, depende de como a superfície foi mergulhada no ambiente.

O tensor curvatura de Riemann é a principal ferramenta para medir curvaturas em uma variedade riemanniana. Ele mede o quanto a métrica $g$ em $M$ difere da métrica euclidiana, isto é, o quanto as métricas não são localmente isométricas.

Definição 1.4.3. Seja $(E, M, \pi)$ um fibrado vetorial com conexão afim $\nabla$. O tensor curvatura de Riemann é definido por

$$
\begin{aligned}
R: \mathfrak{X}(M) \times \mathfrak{X}(M) \times \Gamma(E) & \longleftrightarrow \Gamma(E) \\
(X, Y, \xi) & \longmapsto R(X, Y) \xi
\end{aligned}
$$

onde

$$
R(X, Y) \xi:=\nabla_{X} \nabla_{Y} \xi-\nabla_{Y} \nabla_{X} \xi-\nabla_{[X, Y]} \xi
$$

onde $[\cdot, \cdot]$ denota o colchete (de Lie) de campos.

Para cada $p \in M$ e cada par de vetores tangentes $X_{p}, Y_{p} \in T_{p} M, R(X, Y)$ define um operador linear no espaço das seções suaves do fibrado $E$, em particular, no fibrado tangente $\Gamma(E)=\mathfrak{X}(M)$; $R$ é linear em $X, Y$ e portanto define um tensor. Se $X=\partial_{x_{i}}$ e $Y=\partial_{x_{j}}$ são campos coordenados, $[X, Y]=0$ e $R(X, Y) \xi=\nabla_{X} \nabla_{Y} \xi-\nabla_{Y} \nabla_{X} \xi$. 
O tensor de Riemann mede o grau de não-comutatividade da conexão riemanniana na variedade $M$. De um modo geral, em uma variedade riemanniana, o transporte paralelo de vetores de um ponto $p$ para um ponto $q$ depende do caminho percorrido, ao contrário do que ocorre no espaço euclidiano. As curvaturas são caracterizadas por essa diferença no transporte paralelo.

Para visualizar a situação, veja a figura abaixo. O transporte paralelo do vetor $u$ do ponto $p$ até o ponto $q$ pelo caminho $v w$ resulta em um vetor diferente do vetor obtido se $u$ for transportado pelo caminho $w v$. O tensor de Riemann "mede"essa diferença.

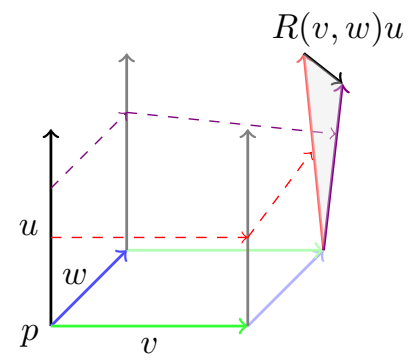

Outra ilustração bastante interessante é a da identidade de Bianchi:

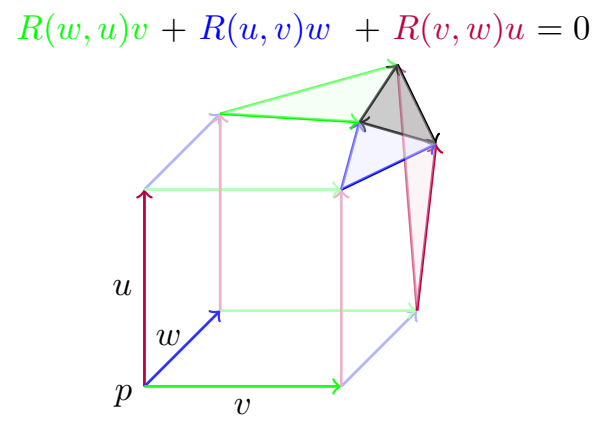

É muito comum escrever $R(X, Y, Z, W)$ para denotar $g(R(X, Y) Z, W)$.

Seja $M$ uma variedade riemanniana de dimensão $m$ e seja $p$ um ponto em $M$. Dado $p \in M$, considere $\Pi$ um subespaço de dimensão 2 do espaço tangente a $M$ em $p$, denotado por $T_{p} M$. Seja $V$ uma vizinhança aberta do zero em $\Pi$ tal que a aplicação exponencial $\exp _{p}$ seja um difeomorfismo entre $V$ e um aberto de $M$. Então $S_{\Pi}=\exp _{p}(V \cap \Pi)$ é uma subvariedade de dimensão 2 de $M$ contendo $p$, chamada de seção plana de $M$ determinada por $\Pi$.

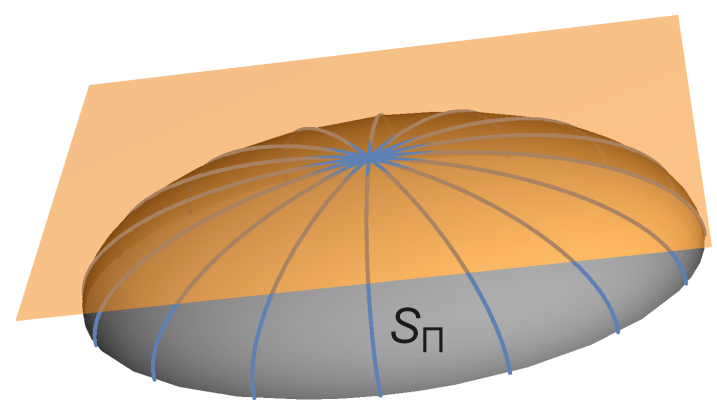

Figura 1.2:

A curvatura seccional de $M$ com respeito ao plano $\Pi, K(\Pi)$, é definida como a curvatura gaussiana da superfície $S_{\Pi}$. É mais comum usarmos a notação $K(X, Y)$, onde $X, Y$ é qualquer 
base de П. A curvatura seccional não depende da base, apenas do plano escolhido e é dada por

$$
K(X, Y):=\frac{g(R(X, Y) X, Y)}{g(X, X) g(Y, Y)-g(X, Y)^{2}}
$$

A curvatura de Ricci de $M$ em um ponto $p$ na direção de um vetor unitário $X_{p} \in T_{p} M$ é a média das curvaturas seccionais com respeito aos planos gerados pelos pares $\left(X_{p}, e_{k}\right), k=2, \ldots, m$ onde $e_{k}$ são os elementos de alguma base ortonormal escolhida $\left\{X_{p}, e_{2}, \ldots, e_{m}\right\}$ de $T_{p} M$, contendo $X_{p}$. A curvatura de Ricci não depende da escolha da base.

Formalmente, o tensor de Ricci é dado pelo o traço do operador simétrico $R(X, \cdot) Y$,

$$
\operatorname{tr} R(X, \cdot) Y=\sum_{k=1}^{m} g\left(R\left(X, e_{k}\right) Y, e_{k}\right)
$$

ou, equivalentemente, pela contração do tensor curvatura riemanniana, e tem coeficientes

$$
\operatorname{Ric}\left(e_{i}, e_{j}\right)=R_{i j}=R_{i k j}^{k}=\sum_{k=1}^{m} g\left(R\left(e_{i}, e_{k}\right) e_{j}, e_{k}\right) .
$$

Tomando $e_{i}=e_{j}=X$, temos

$$
\operatorname{Ric}(X, X)=\sum_{k=2}^{m} K\left(X, e_{k}\right) .
$$

O tensor de Ricci é simétrico e bilinear e a curvatura de Ricci da variedade $M$ no ponto $p$, na direção $X$, é dada por

$$
\operatorname{Ric}_{p}(X)=\frac{1}{m-1} \operatorname{Ric}_{p}(X, X) .
$$

A curvatura escalar de $M$ em um ponto $p$ é a média das curvaturas de Ricci em $p$ na direção de todos os vetores de qualquer base ortonormal do espaço tangente a $M$ em $p$, escolhida,

$$
R(p):=\frac{1}{m} \sum_{i=1}^{m} \operatorname{Ric}_{p}\left(e_{i}\right)
$$

onde $\left\{e_{i}\right\}_{i=1}^{m}$ é base ortonormal do $T_{p} M$. A curvatura escalar é o traço do tensor de Ricci. Ainda, podemos dizer que a curvatura escalar é a média de todas as curvaturas seccionais com respeito aos planos gerados por pares de elementos de uma base ortonormal de $T_{p} M$ :

$$
R(p):=\frac{1}{m} \sum_{i=1}^{m} \operatorname{Ric}_{p}\left(e_{i}, e_{i}\right)=\sum_{i, j=1}^{m} g\left(R\left(e_{i}, e_{j}\right) e_{i}, e_{j}\right)=\sum_{i \neq j} K\left(e_{i}, e_{j}\right) .
$$

A curvatura escalar não depende da escolha da base.

Seja $(\widetilde{M}, \widetilde{g})$ uma variedade riemanniana de dimensão $m$, seja $M$ uma variedade de dimensão $n$ e seja $i: M \longrightarrow \widetilde{M}$ uma imersão (ou mergulho). Se definirmos $g=i^{*} \tilde{g}$ como a métrica riemanniana em $M, i$ é chamada imersão isométrica (ou mergulho isométrico). Se $i$ é uma aplicação injetiva, $M$ é dita subvariedade (imersa ou mergulhada) de $\widetilde{M}$ (chamada de variedade ambiente). Como trataremos de noções locais e toda imersão é, localmente, um mergulho, vamos supor, sem perda de generalidade que $M$ é uma subvariedade mergulhada de $\widetilde{M}$.

É importante lembrar que, em cada ponto $p \in M$, podemos decompor o espaço tangente como $T_{p} \widetilde{M}=T_{p} M \oplus\left(T_{p} M\right)^{\perp}$, onde $\left(T_{p} M\right)^{\perp}$ denota o espaço normal em $p$ com respeito à métrica $\widetilde{g}$ em $T_{p} \widetilde{M}$

Sejam $X, Y$ campos vetoriais suaves em $M$, então podemos estendê-los a campos suaves em $\widetilde{M}$. Tomando a derivada covariante com respeito à métrica $\widetilde{g}$, denotada por $\widetilde{\nabla}$, podemos escrever 
a decomposição

$$
\widetilde{\nabla}_{X} Y=\left(\widetilde{\nabla}_{X} Y\right)^{\top}+\left(\widetilde{\nabla}_{X} Y\right)^{\perp},
$$

em cada ponto $p$ de $M$, onde a componente tangencial é nada mais que $\nabla_{X} Y$, a derivada covariante com respeito a métrica $g$ em $M$. A componente normal é a chamada segunda forma fundamental de $M$, uma forma bilinear, simétrica, assim definida

$$
\begin{array}{rlc}
I I: T M \times T M & \longrightarrow & T M^{\perp} \\
(X, Y) & \longmapsto & \left(\widetilde{\nabla}_{X} Y\right)^{\perp}
\end{array}
$$

Podemos usar a segunda forma fundamental para calcular a derivada covariante de um campo normal $\eta$ à subvariedade $M$, fazendo

$$
\left\langle\widetilde{\nabla}_{X} \eta, Y\right\rangle=-\langle\eta, I I(X, Y)\rangle .
$$

A fórmula de Gauss

$$
\widetilde{\nabla}_{X} Y=\nabla_{X} Y+I I(X, Y),
$$

interpreta a segunda forma fundamental como a diferença entre a conexão com respeito à métrica da variedade ambiente $\widetilde{M}$ e a conexão com respeito à métrica de $M$. Há outras fórmulas como a equação de Gauss que ajudam-nos a compreender que, no fundo, a segundo forma fundamental nos permite entender como curva a subvariedade $M$ com respeito a $\widetilde{M}$. Assim, a curvatura média de $M$, dada pelo traço da segunda forma fundamental, é uma medida extrínseca à subvariedade $M$ e seu sinal depende da escolha do campo unitário normal à $M$.

Lembre que o bordo $\partial M$ de uma variedade com bordo $M$, de dimensão $m$, é uma subvariedade (sem bordo) de $M$, com dimensão $m-1$. Se a curvatura média de $\partial M$ é nula, dizemos que $\partial M$ é mínimo ou minimal. Se a segunda forma fundamental de $\partial M$ é identicamente nula, dizemos que o bordo é totalmente geodésico. É claro que toda hipersuperfície totalmente geodésica é mínima. Contudo existem subvariedades mínimas que não são totalmente geodésicas. Temos um belo exemplo para ilustrar o caso.

Exemplo 1.4.4. O toro de Clifford é uma subvariedade da esfera $\mathbb{S}^{3}$ em $\mathbb{R}^{4}$ : é o produto de dois $\mathbb{S}^{1}$ mergulhados no $\mathbb{R}^{2}$,

$$
\sqrt{\frac{1}{2}} S^{1} \times \sqrt{\frac{1}{2}} S^{1}=\left\{\sqrt{\frac{1}{2}}(\cos \theta, \operatorname{sen} \theta, \cos \phi, \operatorname{sen} \phi), 0 \leq \theta<2 \pi, 0 \leq \phi<2 \pi\right\} .
$$

Mais que isso, o toro de Clifford é uma superfície mínima em $\mathbb{S}^{3}$ que não é totalmente geodésica e que divide $\mathbb{S}^{3}$ em dois toros sólidos de mesmo volume, cada um dos quais é uma variedade com bordo, de dimensão 3 e curvatura constante. De fato, o toro de Clifford é o bordo comum à estas duas variedades. Assim, metade da esfera $\mathbb{S}^{3}$, tendo o toro de Clifford como bordo, é uma variedade com curvatura escalar constante, cujo bordo é mínimo e não totalmente geodésico.

\subsection{Transformações Conformes}

Sejam $\left(M_{1}, g^{(1)}\right)$ e $\left(M_{2}, g^{(2)}\right)$ duas variedades riemannianas. Um difeomorfismo $F: M_{1} \longrightarrow M_{2}$ é dito conforme se o pull-back da métrica $g^{(2)}$ por $f$ é conforme à métrica $g^{(1)}$, isto é, existe uma função suave positiva $\phi$ definida em $M$ tal que $F^{*}\left(g^{(2)}\right)=\phi g^{(1)}$. Se $\phi$ é constante, $F$ é homotetia; se $\phi=i d, F$ é isometria. O grupo de isometrias de $M$, denotado por $I s o(M, g)$, é subgrupo do grupo de aplicações conformes. Aplicações conformes preservam ângulos entre curvas, respeitando sua orientação, e formas de objetos geométricos, localmente, mas não, necessariamente, comprimento ou curvatura. A projeção estereográfica é um exemplo de difeomorfismo conforme.

Definição 1.5.1. A classe conforme de uma métrica $g$, denotada por $[g]$ é o conjunto de métricas $\tilde{g}=\phi g$, onde $\phi$ é uma função suave, positiva, definida em $M$. 
As expressões para a forma volume, conexão riemanniana, curvaturas e outros objetos definidos em uma variedade sofrem alterações quando consideramos uma métrica conforme à métrica dada. Neste capítulo, $(M, g)$ será uma variedade riemanniana compacta de dimensão $m \geq 3$ e $\left(x_{1}, \ldots, x_{m}\right)$ um sistema de coordenadas locais. Consideraremos a métrica ${ }^{3} \tilde{g}=\mathrm{e}^{2 f} g$, onde $f \in \mathcal{C}^{k}(M)$, com $k \geq 3$, na classe conforme de $g$. Então, temos

$$
\begin{aligned}
\tilde{g} & =\tilde{g}_{i j} d x^{i} \otimes d x^{j} \\
& =\left(\mathrm{e}^{2 f} g\right)_{i j} d x^{i} \otimes d x^{j} \\
& =\mathrm{e}^{2 f} g_{i j} d x^{i} \otimes d x^{j}
\end{aligned}
$$

portanto $\tilde{g}_{i j}=\mathrm{e}^{2 f} g_{i j}$ e sua inversa fica $\tilde{g}^{i j}=\mathrm{e}^{-2 f} g^{i j}$. Temos a seguinte expressão para a forma volume associada a $\tilde{g}$ :

$$
\begin{aligned}
\omega_{\tilde{g}} & =\sqrt{\left|\operatorname{det}\left(\tilde{g}_{i j}\right)_{i j}\right|} d x^{1} \wedge \ldots \wedge d x^{m} \\
& =\sqrt{\left|\operatorname{det}\left(\mathrm{e}^{2 f} g_{i j}\right)_{i j}\right|} d x^{1} \wedge \ldots \wedge d x^{m} \\
& =\sqrt{\mathrm{e}^{2 m f}\left|\operatorname{det}\left(g_{i j}\right)_{i j}\right|} d x^{1} \wedge \ldots \wedge d x^{m} \\
& =\mathrm{e}^{m f} \sqrt{\left|\operatorname{det}\left(g_{i j}\right)_{i j}\right|} d x^{1} \wedge \ldots \wedge d x^{m} \\
& =\mathrm{e}^{m f} \omega_{g}
\end{aligned}
$$

\subsubsection{Conexão Riemanniana}

Seja $\nabla$ a conexão riemanniana em $T M$ associada à métrica $g$ da variedade $M$, de dimensão $m$, definida acima, e denote por $\widetilde{\nabla}$ a conexão riemanniana associada à métrica conforme $\tilde{g}=\mathrm{e}^{2 f} g$. Considere $X, Y, Z \in \mathfrak{X}(M)$ campos vetoriais suaves em $M$. Pela fórmula de Koszul,

$$
\begin{aligned}
2 \tilde{g}\left(\widetilde{\nabla}_{X} Y, Z\right) & =X \tilde{g}(Y, Z)+Y \tilde{g}(X, Z)-Z \tilde{g}(X, Z) \\
& -\tilde{g}([X, Y], Z)-\tilde{g}([X, Z], Y)-\tilde{g}([Y, Z], X)
\end{aligned}
$$

Pela regra de Leibniz

$$
X \tilde{g}(Y, Z)=X\left(\mathrm{e}^{2 f} g\right)(Y, Z)=2 \mathrm{e}^{2 f} X(f) g(Y, Z)+\mathrm{e}^{2 f} X g(Y, Z),
$$

$\log 0$

$$
\begin{aligned}
2 \tilde{g}\left(\widetilde{\nabla}_{X} Y, Z\right) & =\mathrm{e}^{2 f}[2 X(f) g(Y, Z)+X g(Y, Z)+2 Y(f) g(X, Z)+Y g(X, Z) \\
& -2 Z(f) g(X, Y)-Z g(X, Y)-g([X, Y], Z)-g([X, Z], Y)-g([Y, Z], X)] \\
2 \tilde{g}\left(\widetilde{\nabla}_{X} Y, Z\right) & =\mathrm{e}^{2 f}[(2 X(f) g(Y, Z)+2 Y(f) g(X, Z)-2 Z(f) g(X, Y)] \\
& +\mathrm{e}^{2 f}[X g(Y, Z)+Y g(X, Z)-Z g(X, Y) \\
& -g([X, Y], Z)-g([X, Z], Y)-g([Y, Z], X)] \\
2 \tilde{g}\left(\widetilde{\nabla}_{X} Y, Z\right) & =2 \mathrm{e}^{2 f}[X(f) g(Y, Z)+Y(f) g(X, Z)-Z(f) g(X, Y)] \\
& +\mathrm{e}^{2 f}\left[2 g\left(\nabla_{X} Y, Z\right)\right]
\end{aligned}
$$

\footnotetext{
${ }^{3} \hat{E}$ usual tomar $\phi=\mathrm{e}^{2 f}>0$ para facilitar as contas, o que não causa perda de generalidade, já que, fazendo $f=\frac{\ln \phi}{2}$ pode-se obter a expressão em termos de $\phi$, facilmente.
} 
Note que $Z(f)=\mathrm{d} f(Z)=g(\nabla f, Z)$, onde $\nabla f:=\operatorname{grad}_{g} f$ é o gradiente da função $f$, então

$$
\begin{aligned}
2 \tilde{g}\left(\widetilde{\nabla}_{X} Y, Z\right) & =2\left[X(f) \mathrm{e}^{2 f} g(Y, Z)+Y(f) \mathrm{e}^{2 f} g(X, Z)-\mathrm{e}^{2 f} g(\nabla f, Z) g(X, Y)\right] \\
& +2 \mathrm{e}^{2 f} g\left(\nabla_{X} Y, Z\right) \\
\not{\not} \tilde{g}\left(\widetilde{\nabla}_{X} Y, Z\right) & =\not{2}[X(f) \tilde{g}(Y, Z)+Y(f) \tilde{g}(X, Z)-\tilde{g}(\nabla f, Z) g(X, Y)]+\not 2 \tilde{g}\left(\nabla_{X} Y, Z\right) \\
& =\tilde{g}(X(f) Y, Z)+\tilde{g}(Y(f) X, Z)-\tilde{g}(g(X, Y) \nabla f, Z)+\tilde{g}\left(\nabla_{X} Y, Z\right) \\
& =\tilde{g}\left(X(f) Y+Y(f) X-g(X, Y) \nabla f+\nabla_{X} Y, Z\right),
\end{aligned}
$$

para qualquer campo $Z \in \mathfrak{X}(M)$. Assim, temos

$$
\widetilde{\nabla}_{X} Y=\nabla_{X} Y+X(f) Y+Y(f) X-g(X, Y) \nabla f \text {. }
$$

\section{Símbolos de Christoffel}

Os símbolos de Christoffel são os coeficientes da conexão riemanniana em um sistema de coordenadas locais. O tensor curvatura de Riemann pode ser completamente descrito em termos dos símbolos de Christoffel, assim como o tensor de Ricci e a curvatura escalar, por tratarem-se de medidas associadas à geometria intrínseca da variedade. Não faremos uso dos símbolos de Christoffel para obtermos estas curvaturas com respeito à uma métrica conforme, embora este seja um meio de se fazer isso, mesmo assim, exibiremos aqui as alterações sofridas pelos símbolos de Christoffel sob transformações conformes. Para $\tilde{g}=\mathrm{e}^{2 f} g$, temos

$$
\begin{aligned}
\widetilde{\Gamma}_{i j}^{k} & =\frac{1}{2} \sum_{k}\left(\frac{\partial}{\partial x_{i}} \tilde{g}_{j k}+\frac{\partial}{\partial x_{j}} \tilde{g}_{k i}-\frac{\partial}{\partial x_{k}} \tilde{g}_{i j}\right) \tilde{g}^{k m} \\
& =\frac{1}{2} \sum_{k}\left(\frac{\partial}{\partial x_{i}}\left(\mathrm{e}^{2 f} g_{j k}\right)+\frac{\partial}{\partial x_{j}}\left(\mathrm{e}^{2 f} g_{k i}\right)-\frac{\partial}{\partial x_{k}}\left(\mathrm{e}^{2 f} g_{i j}\right)\right) \mathrm{e}^{-2 f} g^{k m} \\
& =\frac{1}{2} \sum_{k}\left[2 \mathrm{e}^{2 f} \frac{\partial f}{\partial x_{i}} g_{j k}+\mathrm{e}^{2 f} \frac{\partial}{\partial x_{i}} g_{j k}+2 \mathrm{e}^{2 f} \frac{\partial f}{\partial x_{j}} g_{k i}+\mathrm{e}^{2 f} \frac{\partial}{\partial x_{j}} g_{k i}-\right. \\
& \left.-2 \mathrm{e}^{2 f} \frac{\partial f}{\partial x_{k}} g_{i j}-\mathrm{e}^{2 f} \frac{\partial}{\partial x_{k}} g_{i j}\right] \mathrm{e}^{-2 f} g^{k m} \\
& =\frac{1}{\not 2} \sum_{k} \not \mathrm{e}^{2 f}\left[\frac{\partial f}{\partial x_{i}} g_{j k}+\frac{\partial f}{\partial x_{j}} g_{k i}-\frac{\partial f}{\partial x_{k}} g_{i j}\right] \mathrm{e}^{-2 f} g^{k m} \\
& +\frac{1}{2} \sum_{k} \mathrm{e}^{2 f}\left[\frac{\partial}{\partial x_{i}} g_{j k}+\frac{\partial}{\partial x_{j}} g_{k i}-\frac{\partial}{\partial x_{k}} g_{i j}\right] \mathrm{e}^{-2 f} g^{k m} \\
& =\sum_{k}\left[\frac{\partial f}{\partial x_{i}} g_{j k}+\frac{\partial f}{\partial x_{j}} g_{k i}-\frac{\partial f}{\partial x_{k}} g_{i j}\right] g^{k m} \\
& +\frac{1}{2} \sum_{k}\left[\frac{\partial}{\partial x_{i}} g_{j k}+\frac{\partial}{\partial x_{j}} g_{k i}-\frac{\partial}{\partial x_{k}} g_{i j}\right] g^{k m} \\
& =\sum_{k}\left[\frac{\partial f}{\partial x_{i}} g_{j k} g^{k m}+\frac{\partial f}{\partial x_{j}} g_{k i} g^{k m}-\frac{\partial f}{\partial x_{k}} g_{i j} g^{k m}\right]+\Gamma_{i j}^{k}
\end{aligned}
$$

segue que

$$
\widetilde{\Gamma}_{i j}^{k}=\Gamma_{i j}^{k}+\frac{\partial f}{\partial x_{i}} \delta_{j}^{m}+\frac{\partial f}{\partial x_{j}} \delta_{i}^{m}-\frac{\partial f}{\partial x_{k}} g_{i j} g^{k m},
$$

onde $\delta_{j}^{m}$ é o delta de Kronecker. 


\subsubsection{Curvatura Riemanniana}

Mantendo as notações anteriores, recorde ainda que existe um isomorfismo canônico entre o espaço tangente e o espaço cotangente da variedade $(M, g)$, permitindo a identificação $\mathrm{d} f \equiv \nabla f$. Decorre que

$$
X \cdot f=X(f)=(\mathrm{d} f)(X)=\langle\nabla f, X\rangle_{g}=\nabla_{X} f=\nabla f(X),
$$

$\log 0$

$$
\nabla f(f)=(\mathrm{d} f)(\nabla f)=\langle\nabla f, \nabla f\rangle_{g}=g(\nabla f, \nabla f)=\|\nabla f\|^{2} .
$$

Além disso, o quadrado da derivada covariante de $f$ com respeito aos campos $X, Y$, é dado por

$$
\nabla^{2} f(X, Y)=\nabla_{X} \nabla_{Y} f-\nabla_{\nabla_{X} Y} f
$$

ou, em notação equivalente,

$$
\nabla_{X, Y}^{2} f=X(Y f)-\left(\nabla_{X} Y\right) f
$$

Note que, a hessiana de $f$, hess $f \in \Gamma\left(T^{*} M \oplus T^{*} M\right)$, é o $(0,2)$-tensor definido por

$$
\operatorname{hess}_{f}:=\nabla \nabla f=\nabla \mathrm{d} f=D(\nabla f),
$$

onde,

$$
\begin{aligned}
\nabla \nabla f(X, Y) & =X(Y f)-\left(\nabla_{X} Y\right) f \\
& =X g(\nabla f, Y)-g\left(\nabla f, \nabla_{X} Y\right) \\
& =g\left(\nabla_{X} \nabla f, Y\right)+g\left(\nabla f, \nabla_{X} Y\right)-g(\nabla f, \nabla X Y)
\end{aligned}
$$

isto é, a hessiana de $f$ pode ser expressa como

$$
\operatorname{hess}_{f}(X, Y)=g\left(\nabla_{X} \nabla f, Y\right),
$$

ou, pela expressão (1.3),

$$
\operatorname{hess}_{f}(X, Y)=\nabla \nabla f(X, Y)=\nabla^{2} f(X, Y),
$$

Atenção, $\nabla \nabla f(X, Y) \neq \nabla_{X} \nabla_{Y} f=X(Y f)$. Note, ainda, que podemos escrever

$$
g\left(\nabla_{X} \nabla f, Y\right)=g\left(\operatorname{hess}_{f}(X), Y\right) .
$$

Visto isto, podemos proceder ao cálculo da curvatura riemanniana com respeito à métrica conforme $\tilde{g}$. Temos

$$
\widetilde{R}(X, Y) Z=\widetilde{\nabla}_{X} \widetilde{\nabla}_{Y} Z-\widetilde{\nabla}_{Y} \widetilde{\nabla}_{X} Z-\widetilde{\nabla}_{[X, Y]} Z,
$$

pela seção anterior, sabemos que

$$
\widetilde{\nabla}_{Y} Z=\nabla_{Y} Z+Y(f) Z+Z(f) Y-g(Y, Z) \nabla f,
$$

$\log 0$

$$
\begin{aligned}
\widetilde{\nabla}_{X} \widetilde{\nabla}_{Y} Z & =\widetilde{\nabla}_{X} \nabla_{Y} Z+\widetilde{\nabla}_{X} Y(f) Z+\widetilde{\nabla}_{X} Z(f) Y-\widetilde{\nabla}_{X} g(Y, Z) \nabla f \\
& =\nabla_{X} \nabla_{Y} Z+X(f) \nabla_{Y} Z+\nabla_{Y} Z(f) X-g\left(X, \nabla_{Y} Z\right) \nabla f \\
& +X Y(f) Z+Y(f) \widetilde{\nabla}_{X} Z+X Z(f) Y+Z(f) \widetilde{\nabla}_{X} Y \\
& -X g(Y, Z) \nabla f-g(Y, Z) \widetilde{\nabla}_{X}(\nabla f)
\end{aligned}
$$




$$
\begin{aligned}
\widetilde{\nabla}_{X} \widetilde{\nabla}_{Y} Z & =\nabla_{X} \nabla_{Y} Z+X(f) \nabla_{Y} Z+\nabla_{Y} Z(f) X-g\left(X, \nabla_{Y} Z\right) \nabla f \\
& +X Y(f) Z+Y(f) \nabla_{X} Z+Y(f) X(f) Z+Y(f) Z(f) X-Y(f) g(X, Z) \nabla f \\
& +X Z(f) Y+Z(f) \nabla_{X} Y+Z(f) X(f) Y+Z(f) Y(f) X-Z(f) g(X, Y) \nabla f \\
& -X g(Y, Z) \nabla f-g(Y, Z)[\underbrace{\nabla_{X}(\nabla f)}_{\operatorname{hess}_{f}(X)}+\underbrace{X(f) \nabla f}_{\underline{q(\nabla f) \nabla f}}+\underbrace{\nabla f(f) X}_{\|\nabla f\|^{2} X}-\underline{g(X, \nabla f) \nabla f]} \\
\widetilde{\nabla}_{X} \widetilde{\nabla}_{Y} Z & =\nabla_{X} \nabla_{Y} Z+X(f) \nabla_{Y} Z+\nabla_{Y} Z(f) X-g\left(X, \nabla_{Y} Z\right) \nabla f \\
& +X Y(f) Z+Y(f) \nabla_{X} Z+Y(f) X(f) Z+Y(f) Z(f) X-Y(f) g(X, Z) \nabla f \\
& +X Z(f) Y+Z(f) \nabla_{X} Y+Z(f) X(f) Y+Z(f) Y(f) X-Z(f) g(X, Y) \nabla f \\
& -X g(Y, Z) \nabla f-g(Y, Z) \operatorname{hess}_{f}(X)-g(Y, Z)\|\nabla f\|^{2} X .
\end{aligned}
$$

Analogamente,

$$
\begin{aligned}
\widetilde{\nabla}_{Y} \widetilde{\nabla}_{X} Z & =\nabla_{Y} \nabla_{X} Z+Y(f) \nabla_{X} Z+\nabla_{X} Z(f) Y-g\left(Y, \nabla_{X} Z\right) \nabla f \\
& +Y X(f) Z+X(f) \nabla_{Y} Z+X(f) Y(f) Z+X(f) Z(f) Y-X(f) g(Y, Z) \nabla f \\
& +Y Z(f) X+Z(f) \nabla_{Y} X+Z(f) Y(f) X+Z(f) X(f) Y-Z(f) g(Y, X) \nabla f \\
& -Y g(X, Z) \nabla f-g(X, Z) \operatorname{hess}_{f}(Y)-g(X, Z)\|\nabla f\|^{2} Y .
\end{aligned}
$$

Além disso,

$$
\widetilde{\nabla}_{[X, Y]} Z=\nabla_{[X, Y]} Z+[X, Y](f) Z+Z(f)[X, Y]-g([X, Y], Z) \nabla f
$$

implica em

$$
\begin{aligned}
\widetilde{\nabla}_{[X, Y]} Z & =\nabla_{[X, Y]} Z+X(Y(f)) Z-Y(X(f)) Z+Z(f) \nabla_{X} Y-Z(f) \nabla_{Y} X \\
& -g\left(\nabla_{X} Y, Z\right) \nabla f+g\left(\nabla_{Y} X, Z\right) \nabla f .
\end{aligned}
$$

Agora, podemos usar as expressões obtidas para calcularmos $\widetilde{R}(X, Y) Z$. Combinando a primeira parcela de $\widetilde{\nabla}_{[X, Y]} Z, \widetilde{\nabla}_{X} \widetilde{\nabla}_{Y} Z$ e $\widetilde{\nabla}_{Y} \widetilde{\nabla}_{X} Z$, com os sinais apropriados, obtemos $R(X, Y) Z$. É fácil ver que quatro termos, em cada expressão, cancelam-se diretamente, além disso, como $Y(f) X(f)=X(f) Y(f)$ e usando a simetria da métrica, cancelamos outros dois termos nas duas primeiras expressões. Pela propriedade de compatibilidade com a métrica da conexão riemanniana, segue que

$-g\left(X, \nabla_{Y} Z\right) \nabla f+g\left(Y, \nabla_{X} Z\right) \nabla f-X g(Y, Z) \nabla f+Y g(X, Z) \nabla f+g\left(\nabla_{X} Y, Z\right) \nabla f-g\left(\nabla_{Y} X, Z\right) \nabla f=0$.

Finalmente, usando (1.3) ficamos com

$\begin{aligned} \widetilde{R}(X, Y) Z & =R(X, Y) Z \\ & -\left[\nabla^{2} f(Y, Z)-Y(f) Z(f)+g(Y, Z)\|\nabla f\|^{2}\right] X \\ & +\left[\nabla^{2} f(X, Z)-X(f) Z(f)+g(X, Z)\|\nabla f\|^{2}\right] Y \\ & -g(Y, Z) \operatorname{hess}_{f}(X)+g(X, Z) \operatorname{hess}_{f}(Y) \\ & -[Y(f) g(X, Z)-X(f) g(Y, Z)] \nabla f\end{aligned}$

\subsubsection{Curvatura de Ricci}

Seja $\left\{e_{i}\right\}_{i=1}^{m}$ um referencial ortonormal em $T M$ com respeito à métrica $g$, então a relação $\widetilde{e}_{i}=\mathrm{e}^{-f} e_{i}$, válida para todo $i$, dá origem a um referencial ortonormal em $T M$ com respeito à 
métrica $\tilde{g}$. Por definição, a curvatura de Ricci de $M$ com respeito a $\tilde{g}$ é dada por

$$
\operatorname{Ric}_{\tilde{g}}(Y, Z)=\sum_{i=1}^{m} \tilde{g}\left(\widetilde{R}\left(\widetilde{e}_{i}, Y\right) Z, \widetilde{e}_{i}\right)
$$

isto é,

$$
\begin{gathered}
\operatorname{Ric}_{\tilde{g}}(Y, Z)=\sum_{i=1}^{m} \mathrm{e}^{2 f} g\left(\widetilde{R}\left(\mathrm{e}^{-f} e_{i}, Y\right) Z, \mathrm{e}^{-f} e_{i}\right)=\mathrm{e}^{2 f} \mathrm{e}^{-2 f} \sum_{i=1}^{m} g\left(\widetilde{R}\left(e_{i}, Y\right) Z, e_{i}\right) \\
\operatorname{Ric}_{\tilde{g}}(Y, Z)=\sum_{i=1}^{m} g\left(\widetilde{R}\left(e_{i}, Y\right) Z, e_{i}\right)
\end{gathered}
$$

Substituindo a expressão obtida para o tensor curvatura riemanniana, temos

$$
\begin{aligned}
\operatorname{Ric}_{\tilde{g}}(Y, Z) & =\sum_{i=1}^{m} g\left(R\left(e_{i}, Y\right) Z\right. \\
& -\left[\nabla^{2} f(Y, Z)-Y(f) Z(f)+g(Y, Z)\|\nabla f\|^{2}\right] e_{i} \\
& +\left[\nabla^{2} f\left(e_{i}, Z\right)-e_{i}(f) Z(f)+g\left(e_{i}, Z\right)\|\nabla f\|^{2}\right] Y \\
& -g(Y, Z) \operatorname{hess}_{f}\left(e_{i}\right)+g\left(e_{i}, Z\right) \operatorname{hess}_{f}(Y) \\
& \left.-\left[Y(f) g\left(e_{i}, Z\right)-e_{i}(f) g(Y, Z)\right] \nabla f, e_{i}\right) .
\end{aligned}
$$

Vamos analisar as parcelas acima, separadamente. Antes, lembre-se que, em um referência local, temos

$$
\begin{array}{ll}
\nabla f=\sum_{i} e_{i}(f) e_{i}, & \text { (Gradiente) } \\
\operatorname{div}_{g}(X)=\sum_{i} g\left(\nabla_{e_{i}} X, e_{i}\right), & \text { (Divergente) } \\
\Delta f=-\operatorname{div}_{g}(\nabla f)=-\sum_{i} g\left(\nabla_{e_{i}} \nabla f, e_{i}\right), & \text { (Laplaciano) }
\end{array}
$$

e observe que, combinando a expressão para o operador laplaciano com a equação (1.6), temos

$$
\Delta f=-\sum_{i} g\left(\nabla_{e_{i}} \nabla f, e_{i}\right)=-\sum_{i} g\left(\operatorname{hess}_{f}\left(e_{i}\right), e_{i}\right)=-\operatorname{tr}_{g} \operatorname{hess}_{f} .
$$

Agora, analisemos as parcelas do Ricci de $\tilde{g}$ :

1. $\sum_{i} g\left(R\left(e_{i}, Y\right) Z, e_{i}\right)=\operatorname{Ric}_{g}(Y, Z)$;

2. $\sum_{i} g\left(\nabla^{2} f\left(e_{i}, Z\right) Y, e_{i}\right)=\sum_{i} \nabla^{2} f\left(e_{i}, Z\right) g\left(Y, e_{i}\right)$

$$
\begin{aligned}
& =\sum_{i} g\left(\operatorname{hess}_{f}\left(e_{i}\right), Z\right) g\left(Y, e_{i}\right) \\
& =\sum_{i} g\left(\operatorname{hess}_{f}\left(e_{i}\right), Z\right) Y_{i} \\
& =\sum_{i} g\left(\operatorname{hess}_{f}\left(e_{i}\right) Y_{i}, Z\right) \\
& =g\left(\operatorname{hess}_{f}(Y), Z\right) \\
& =\nabla^{2} f(Y, Z)
\end{aligned}
$$

observe que hess ${ }_{f}\left(e_{i}\right)$ é a $i$-ésima coluna da matriz hessiana de $f$ e $Y_{i}$ é a $i$-ésima componente 
do campo $Y$;

3. $\sum_{i} g\left(e_{i}(f) Z(f) Y, e_{i}\right)=\sum_{i} e_{i}(f) Z(f) g\left(Y, e_{i}\right)$

$$
\begin{aligned}
& =\sum_{i} g\left(\nabla f, e_{i}\right) g(\nabla f, Z) g\left(Y, e_{i}\right) \\
& =g(\nabla f, Y) g(\nabla f, Z) \\
& =Y(f) Z(f) ;
\end{aligned}
$$

4. $\sum_{i} g\left(g\left(e_{i}, Z\right)\|\nabla f\|^{2} Y, e_{i}\right)=\sum_{i} g\left(e_{i}, Z\right)\|\nabla f\|^{2} g\left(Y, e_{i}\right)$

$$
=g(Y, Z)\|\nabla f\|^{2}
$$

5. $\sum_{i} g\left(g(Y, Z) \operatorname{hess}_{f}\left(e_{i}\right), e_{i}\right)=\sum_{i} g(Y, Z) g\left(\operatorname{hess}_{f}\left(e_{i}\right), e_{i}\right)$

$$
\begin{aligned}
& =g(Y, Z) \operatorname{tr}_{g} \operatorname{hess}_{f} \\
& =-g(Y, Z) \Delta f
\end{aligned}
$$

6. $\sum_{i} g\left(g\left(e_{i}, Z\right) \operatorname{hess}_{f}(Y), e_{i}\right)=\sum_{i} g\left(e_{i}, Z\right) g\left(\operatorname{hess}_{f}(Y), e_{i}\right)$

$$
\begin{aligned}
& =g\left(\operatorname{hess}_{f}(Y), Z\right) \\
& =\nabla^{2} f(Y, Z)
\end{aligned}
$$

a última igualdade decorre da combinação das expressões (1.6), (1.5) e (1.4);

7. $\sum_{i} g\left(Y(f) g\left(e_{i}, Z\right) \nabla f, e_{i}\right)=\sum_{i} Y(f) g\left(e_{i}, Z\right) g\left(\nabla f, e_{i}\right)$

$$
\begin{aligned}
& =Y(f) g(\nabla f, Z) \\
& =Y(f) Z(f)
\end{aligned}
$$

8. $\sum_{i} g\left(e_{i}(f) g(Y, Z) \nabla f, e_{i}\right)=\sum_{i} e_{i}(f) g(Y, Z) g\left(\nabla f, e_{i}\right)$

$$
\begin{aligned}
& =\sum_{i} g\left(\nabla f, e_{i}\right) g(Y, Z) g\left(\nabla f, e_{i}\right) \\
& =g(Y, Z) g(\nabla f, \nabla f) \\
& =g(Y, Z)\|\nabla f\|^{2}
\end{aligned}
$$

As parcelas da segunda linha do Ricci tem resultados triviais. Assim, temos

$$
\begin{aligned}
\operatorname{Ric}_{\tilde{g}}(Y, Z) & =\operatorname{Ric}_{g}(Y, Z)-m \nabla^{2} f(Y, Z)+m Y(f) Z(f)-m g(Y, Z)\|\nabla f\|^{2} \\
& +\nabla^{2} f(Y, Z)-Y(f) Z(f)+g(Y, Z)\|\nabla f\|^{2} \\
& +g(Y, Z) \Delta f+\nabla^{2} f(Y, Z)-Y(f) Z(f)+g(Y, Z)\|\nabla f\|^{2},
\end{aligned}
$$

donde segue

$$
\begin{aligned}
\operatorname{Ric}_{\tilde{g}}(Y, Z) & =\operatorname{Ric}_{g}(Y, Z)-(m-2) \nabla^{2} f(Y, Z)+(m-2) Y(f) Z(f) \\
& -(m-2) g(Y, Z)\|\nabla f\|^{2}+g(Y, Z) \Delta f
\end{aligned}
$$




\subsubsection{Curvatura Escalar}

A curvatura escalar de $M$ com respeito à métrica $\tilde{g}$ é o traço do tensor de Ricci para $\tilde{g}$, calculado acima:

$$
R_{\tilde{g}}=\sum_{i} \operatorname{Ric}_{\tilde{g}}\left(\widetilde{e}_{i}, \widetilde{e}_{i}\right)
$$

onde $\widetilde{e}_{i}$ é o referencial ortonormal com respeito à $\tilde{g}$ definido na seção anterior. Assim, temos

$$
\begin{aligned}
R_{\tilde{g}} & =\sum_{i} \operatorname{Ric}_{\tilde{g}}\left(\mathrm{e}^{-f} e_{i}, \mathrm{e}^{-f} e_{i}\right) \\
& =\mathrm{e}^{-2 f} \sum_{i} \operatorname{Ric}_{\tilde{g}}\left(e_{i}, e_{i}\right) \\
& =\mathrm{e}^{-2 f} \sum_{i}\left[\operatorname{Ric}_{g}\left(e_{i}, e_{i}\right)-(m-2) \nabla^{2} f\left(e_{i}, e_{i}\right)+(m-2) e_{i}(f) e_{i}(f)\right. \\
& \left.-(m-2) g\left(e_{i}, e_{i}\right)\|\nabla f\|^{2}+g\left(e_{i}, e_{i}\right) \Delta f\right] \\
& =\mathrm{e}^{-2 f}\left[R_{g}+(m-2) \Delta f+(m-2)\|\nabla f\|^{2}-(m-2) m\|\nabla f\|^{2}+m \Delta f\right],
\end{aligned}
$$

isto é, a expressão para a curvatura escalar para a métrica conforme é dada por

$$
R_{\tilde{g}}=\mathrm{e}^{-2 f}\left[R_{g}+2(m-1) \Delta f-(m-2)(m-1)\|\nabla f\|^{2}\right]
$$

Agora, se tomarmos $\tilde{g}=\phi^{\frac{4}{m-2}} g, \phi>0$, obteremos uma expressão para a curvatura escalar em função de $\phi$. Note que ao fazermos $\mathrm{e}^{2 f}=\phi^{\frac{4}{m-2}}$, obtemos

$$
\begin{aligned}
\ln \mathrm{e}^{2 f} & =\ln \phi^{\frac{4}{m-2}} \\
2 f & =\frac{4}{m-2} \ln \phi \\
f & =\frac{2}{m-2} \ln \phi
\end{aligned}
$$

e, portanto,

$$
\nabla f=\frac{2}{m-2} \frac{\nabla \phi}{\phi} .
$$

Além disso,

$$
\begin{aligned}
\Delta f & =-\operatorname{div}_{g}(\nabla f) \\
& =-\frac{2}{m-2} \sum_{i} g\left(\nabla_{e_{i}}\left(\frac{\nabla \phi}{\phi}\right), e_{i}\right) \\
& =-\frac{2}{m-2} \sum_{i} g\left(\frac{\nabla_{e_{i}}(\nabla \phi)}{\phi}-\frac{e_{i}(\phi) \nabla \phi}{\phi^{2}}, e_{i}\right) \\
& =-\frac{2}{m-2} \sum_{i}\left[\frac{g\left(\nabla_{e_{i}} \nabla \phi, e_{i}\right)}{\phi}-\frac{e_{i}(\phi) g\left(\nabla \phi, e_{i}\right)}{\phi^{2}}\right] \\
& =-\frac{2}{m-2}\left[-\frac{\Delta \phi}{\phi}-\frac{\|\nabla \phi\|^{2}}{\phi^{2}}\right] \\
& =\frac{2}{m-2}\left[\frac{\Delta \phi}{\phi}+\frac{\|\nabla \phi\|^{2}}{\phi^{2}}\right] .
\end{aligned}
$$


Substituindo na expressão que temos para a curvatura escalar da métrica conforme, temos

$$
\begin{aligned}
R_{\tilde{g}} & =\phi^{-\frac{4}{m-2}}\left[R_{g}+2(m-1) \frac{2}{m-2}\left[\frac{\Delta \phi}{\phi}+\frac{\|\nabla \phi\|^{2}}{\phi^{2}}\right]-(m-2)(m-1)\left\|\frac{2}{m-2} \frac{\nabla \phi}{\phi}\right\|^{2}\right] \\
& =\phi^{-\frac{4}{m-2}}\left[R_{g}+2(m-1) \frac{2}{m-2} \frac{\Delta \phi}{\phi}+2(m-1) \frac{2}{m-2} \frac{\|\nabla \phi\|^{2}}{\phi^{2}}-(m-2)(m-1) \frac{4}{(m-2)^{2}} \frac{\|\nabla \phi\|^{2}}{\phi^{2}}\right] \\
& =\phi^{-\frac{m+2}{m-2}} \phi\left[R_{g}+\frac{4(m-1)}{m-2} \frac{\Delta \phi}{\phi}+\frac{4(m-1)\|\nabla \phi\|^{2}}{\phi^{2}}-\frac{4(m-1)\|\nabla \phi\|^{2}}{(m-2)}\right] \\
& =\phi^{-\frac{m+2}{m-2}}\left[R_{g} \phi+\frac{4(m-1)}{m-2} \Delta \phi\right] .
\end{aligned}
$$

Agora, observe que existe $\tilde{g}$ com curvatura escalar constante, se e somente se, existe $\phi>0$ tal que

$$
\phi^{-\frac{m+2}{m-2}}\left[R_{g} \phi+\frac{4(m-1)}{m-2} \Delta \phi\right]=k
$$

com $k=R_{\tilde{g}}$ constante; ou, equivalentemente,

$$
\frac{(m-2)}{4(m-1)} R_{g} \phi+\Delta \phi=\frac{(m-2)}{4(m-1)} k \phi^{\frac{m+2}{m-2}},
$$

isto é,

$$
\Delta \phi+\frac{(m-2)}{4(m-1)} R_{g} \phi=K \phi^{\frac{m+2}{m-2}}
$$

onde $K$ é constante. Esta última expressão é a equação (2.2) que modela o conhecido problema de Yamabe, como veremos na Seção 2.1 .

\subsubsection{Curvatura Média}

É importante lembrar que a curvatura média é uma noção extrínseca; é uma medida associada a uma subvariedade de uma variedade diferenciável, que depende do particular mergulho que a descreve.

Seja $(M, g)$ uma variedade riemanniana com bordo, de dimensão $m \geq 3$, então o bordo de $M$, $\partial M$ é uma hipersuperfície de $M$, isto é, uma subvariedade de dimensão $m-1$. Seja $N$ um campo unitário (interior) normal ao bordo de $M$, com respeito à métrica $g$. Denotaremos também por $g$ a restrição da métrica ao bordo. Seja $\tilde{g}=\mathrm{e}^{2 f} g$, então a segunda forma fundamental associada a $\partial M$ com respeito à métrica $\tilde{g}$ é

$$
I_{\tilde{g}}(X, Y)=\tilde{g}\left(X, \widetilde{\nabla}_{Y} \tilde{N}\right),
$$

onde $X, Y$ são campos tangentes a $\partial M, \widetilde{N}=\mathrm{e}^{-f} N$ é o campo unitário (interior) normal a $\partial M$, com respeito à métrica $\tilde{g}$. Assim, temos

$$
\begin{aligned}
I_{\tilde{g}}(X, Y) & =\mathrm{e}^{2 f} g\left(X, \widetilde{\nabla}_{Y}\left(\mathrm{e}^{-f} N\right)\right) \\
& =\mathrm{e}^{2 f} g\left(X,-\mathrm{e}^{-f} Y(f) N+\mathrm{e}^{-f} \widetilde{\nabla}_{Y} N\right) \\
& =-Y(f) \mathrm{e}^{f} g(X, X)+\mathrm{e}^{f} g\left(X, \widetilde{\nabla}_{Y} N\right) \\
& =\mathrm{e}^{f} g\left(X, \widetilde{\nabla}_{Y} N\right)
\end{aligned}
$$

Agora, observe que

$$
\widetilde{\nabla}_{Y} N=\nabla_{Y} N+Y(f) N+N(f) Y-g(Y, N) \nabla^{0} f
$$


e portanto,

$$
\begin{aligned}
I I_{\tilde{g}}(X, Y) & =\mathrm{e}^{f} g\left(X, \nabla_{Y} N\right)+Y(f) \mathrm{e}^{f} g\left(X, X+N(f) \mathrm{e}^{f} g(X, Y)\right. \\
& =\mathrm{e}^{f} I I_{g}(X, Y)+N(f) \mathrm{e}^{f} g(X, Y) \\
& I I_{\tilde{g}}(X, Y)=\mathrm{e}^{f}\left[I I_{g}(X, Y)+\partial_{N}(f) g(X, Y)\right]
\end{aligned}
$$

Agora, podemos calcular a curvatura média do bordo: seja $\left\{\widetilde{e}_{i}\right\}$ o referencial ortonormal com respeito a $\tilde{g}$ que já definimos no início da Seção 1.5.3, então

$$
\begin{aligned}
H_{\tilde{g}} & =\operatorname{tr}_{\tilde{g}}\left(I I_{\tilde{g}}\right) \\
& =\tilde{g}^{i j}\left(I I_{\tilde{g}}\right)_{i j} \\
& =\tilde{g}^{i j}\left(I I_{\tilde{g}}\right)\left(e_{i}, e_{j}\right) \\
& =\mathrm{e}^{-2 f} g^{i j} \mathrm{e}^{f}\left[I_{g}\left(e_{i}, e_{j}\right)+\partial_{N}(f) g\left(e_{i}, e_{j}\right)\right] \\
& =\mathrm{e}^{-f}\left[g^{i j}\left(I I_{g}\right)_{i j}+\partial_{N}(f) g^{i j} g_{i j}\right] \\
& =\mathrm{e}^{-f}\left[H_{g}+\partial_{N}(f)(m-1)\right]
\end{aligned}
$$

Observação 1.5.2. A métrica g que aparece acima é a restrição da métrica $g$ em $M$ ao bordo de $M$, por esta razão, o traço da métrica é igual a $m-1$ e não $m$.

Assim, a curvatura média do bordo, sob uma transformação conforme, é dada por

$$
H_{\tilde{g}}=\mathrm{e}^{-f}\left[H_{g}+(m-1) \partial_{N}(f)\right]
$$

Se fizermos $\mathrm{e}^{2 f}=\phi^{\frac{4}{m-2}}$, temos

$$
\begin{aligned}
H_{\tilde{g}} & =\phi^{-\frac{2}{m-2}}\left[H_{g}+(m-1) \partial_{N}\left(\frac{2}{m-2} \ln \phi\right)\right] \\
& =\phi^{-\frac{2}{m-2}}\left[H_{g}+\frac{2(m-1)}{m-2} \frac{\partial_{N}(\phi)}{\phi}\right]
\end{aligned}
$$

logo,

$$
H_{\tilde{g}}=\phi^{-\frac{m}{m-2}}\left[H_{g} \phi+\frac{2(m-1)}{m-2} \partial_{N}(\phi)\right]
$$

\subsubsection{Operador Laplaciano}

O operador laplaciano $\Delta_{g}$ em uma variedade riemanniana $(M, g)$ também sofre alterações sob a ação de transformações conformes em $M$. Nas contas abaixo, para não carregar a notação, 
denotaremos também por $g$ a matriz do tensor métrico $\left(g_{i j}\right)_{i j}$.

$$
\begin{aligned}
\Delta_{\tilde{g}} & =-\frac{1}{\sqrt{|\operatorname{det} \tilde{g}|} \frac{\partial}{\partial x_{j}}}\left(\sqrt{|\operatorname{det} \tilde{g}|} \tilde{g}^{i j} \frac{\partial}{\partial x_{i}}\right) \\
& =-\frac{1}{\sqrt{\left|\operatorname{det} \mathrm{e}^{2 f} g\right|}} \frac{\partial}{\partial x_{j}}\left(\sqrt{\left|\operatorname{det} \mathrm{e}^{2 f} g\right|}\left(\mathrm{e}^{2 f} g\right)^{i j} \frac{\partial}{\partial x_{i}}\right) \\
& =-\frac{1}{\sqrt{\mathrm{e}^{2 m f}|\operatorname{det} g|}} \frac{\partial}{\partial x_{j}}\left(\sqrt{\mathrm{e}^{2 m f}|\operatorname{det} g|} \mathrm{e}^{-2 f} g^{i j} \frac{\partial}{\partial x_{i}}\right) \\
& =-\frac{1}{\mathrm{e}^{m f} \sqrt{|\operatorname{det} g|}} \frac{\partial}{\partial x_{j}}\left(\mathrm{e}^{m f} \sqrt{|\operatorname{det} g|} \mathrm{e}^{-2 f} g^{i j} \frac{\partial}{\partial x_{i}}\right) \\
& =-\frac{1}{\mathrm{e}^{m f} \sqrt{|\operatorname{det} g|}} \frac{\partial}{\partial x_{j}}\left(\mathrm{e}^{(m-2) f} \sqrt{|\operatorname{det} g|} g^{i j} \frac{\partial}{\partial x_{i}}\right) \\
& =\frac{\mathrm{e}^{(m-2) f}}{\mathrm{e}^{m f}} \frac{-1}{\sqrt{|\operatorname{det} g|}} \frac{\partial}{\partial x_{j}}\left(\sqrt{|\operatorname{det} g|} g^{i j} \frac{\partial}{\partial x_{i}}\right) \\
& -\frac{1}{\mathrm{e}^{m f} \sqrt{|\operatorname{tet} g|}}\left(\frac{\partial}{\partial x_{j}} \mathrm{e}^{(m-2) f}\right) \sqrt{|t+t g|} g^{i j} \frac{\partial}{\partial x_{i}} \\
& =\mathrm{e}^{-2 f} \Delta_{g}-\frac{1}{\mathrm{e}^{m f}}(m-2) \mathrm{e}^{(m-2) f} \frac{\partial}{\partial x_{j}} f g^{i j} \frac{\partial}{\partial x_{i}} \\
& =\mathrm{e}^{-2 f} \Delta_{g}-\mathrm{e}^{-2 f}(m-2) \frac{\partial}{\partial x_{j}} f \frac{\partial}{\partial x_{i}}
\end{aligned}
$$

isto é,

$$
\Delta_{\tilde{g}}=\mathrm{e}^{-2 f}\left(\Delta_{g}-(m-2) \frac{\partial f}{\partial x_{j}} \frac{\partial}{\partial x_{j}}\right)
$$

\subsubsection{Variedade Produto}

Na Seção 2.3.3 estudamos uma família de métricas $\bar{g}_{s}=g^{(1)} \oplus s g^{(2)}, s>0$, na variedade produto com bordo $M=M_{1} \times M_{2}$, de dimensão $m=m_{1}+m_{2} \geq 3$, com $M_{1}$ compacta sem bordo e $M_{2}$ compacta com bordo. Ali fazemos um série de afirmações sobre o comportamento das curvaturas escalar e média, sobre o laplaciano e seus autovalores em $M$, nesta seção, veremos a origem de tais afirmações.

\section{Homotetias da Métrica Riemanniana}

Seja $(M, g)$ uma variedade riemanniana compacta com bordo. Antes de vermos o caso das variedades produto, vamos observar, como caso particular dos resultados obtidos na seção anterior, o que acontece com quando fazemos $\mathrm{e}^{2 f}=s>0$. De início, temos

$$
\begin{aligned}
f & =\frac{\ln s}{2} \text { (constante), } \\
\mathrm{e}^{-2 f} & =\frac{1}{s} \\
\sqrt{\mathrm{e}^{2 f}} & =\mathrm{e}^{f}=\sqrt{s} \\
\mathrm{e}^{m f} & =s^{\frac{m}{2}}
\end{aligned}
$$

daí, para $\tilde{g}=s g$, a forma volume fica

$$
\omega_{\tilde{g}}=s^{\frac{m}{2}} \omega_{g},
$$


a conexão Levi-Civita e os símbolos de Christoffel e o (1,3)-tensor de Riemann ficam invariantes

$$
\begin{aligned}
\widetilde{\nabla}_{X} Y & =\nabla_{X} Y, \\
\widetilde{\Gamma}_{i j}^{k} & =\Gamma_{i j}^{k}, \\
\widetilde{R}(X, Y) Z & =R(X, Y) Z .
\end{aligned}
$$

Para o tensor de Riemann, podemos tirar a prova desse resultado, fazendo,

$$
\begin{aligned}
\widetilde{R}_{i j k}^{l} & =\sum_{r} \widetilde{\Gamma}_{i k}^{r} \widetilde{\Gamma}_{j r}^{l}-\sum_{r} \widetilde{\Gamma}_{j k}^{r} \widetilde{\Gamma}_{i r}^{l}+\frac{\partial}{\partial x_{j}} \widetilde{\Gamma}_{i k}^{l}-\frac{\partial}{\partial x_{i}} \widetilde{\Gamma}_{j k}^{l} \\
& =\sum_{r} \Gamma_{i k}^{r} \Gamma_{j r}^{l}-\sum_{r} \Gamma_{j k}^{r} \Gamma_{i r}^{l}+\frac{\partial}{\partial x_{j}} \Gamma_{i k}^{l}-\frac{\partial}{\partial x_{i}} \Gamma_{j k}^{l} \\
& =R_{i j k}^{l}
\end{aligned}
$$

porém, vale observar que o mesmo não ocorre para o $(0,4)$-tensor de Riemann,

$$
\begin{aligned}
\widetilde{R}_{i j k l} & =\sum_{m} \widetilde{R}_{i j k}^{m} \widetilde{g}_{m l} \\
& =\sum_{m} R_{i j k}^{m} s g_{m l} \\
& =s \sum_{m} R_{i j k}^{m} g_{m l} \\
& =s R_{i j k l},
\end{aligned}
$$

isto é, $\widetilde{R}(X, Y, Z, W)=s R(X, Y, Z, W)$.

Note que, se $\left\{e_{i}\right\}_{i=1}^{n}$ é um referencial ortonormal para o espaço tangente com respeito a $g$, então $\left\{\widetilde{e}_{i}=\frac{1}{\sqrt{s}} e_{i}\right\}_{i=1}^{n}$ é um referencial ortonormal com respeito a $\widetilde{g}$,

$$
\widetilde{g}\left(\widetilde{e_{i}}, \widetilde{e}_{i}\right)=s g\left(\widetilde{e_{i}}, \widetilde{e}_{i}\right)=s \frac{1}{s} g\left(e_{i}, e_{i}\right)=1
$$

e

$$
\operatorname{Ric}_{\tilde{g}}=R i c_{g}
$$

A curvatura escalar, fica

$$
R_{\tilde{g}}=\frac{1}{s} R_{g}
$$

Analogamente, $\tilde{N}=\frac{1}{\sqrt{s}} N$ é respectivo campo unitário (interior) normal ao bordo de $M$ e a segunda forma fundamental, bem como a curvatura média do bordo ficam

$$
\begin{aligned}
I_{\tilde{g}}(X, Y) & =\sqrt{s} I_{g}(X, Y), \\
H_{\tilde{g}} & =\frac{1}{\sqrt{s}} H_{g} .
\end{aligned}
$$


O operador laplaciano fica $\widetilde{\Delta}(f)=\frac{1}{s} \Delta(f)$

$$
\begin{aligned}
\widetilde{\Delta}(f) & =\widetilde{\nabla}_{i} \widetilde{\nabla}^{i}(f)=\nabla_{i}\left(\nabla_{j}(f) \widetilde{g}^{i j}\right) \\
& =\nabla_{i}\left(\nabla_{j}(f) \frac{1}{s} g^{i j}\right)=\nabla_{i}\left(\frac{1}{s} \nabla^{i}(f)\right) \\
& =\frac{1}{s} \nabla_{i} \nabla^{i}(f)=\frac{1}{s} \Delta(f),
\end{aligned}
$$

portanto, se $\lambda$ é um autovalor de $\Delta$ associado a $f$, então $\lambda$ é autovalor de $\widetilde{\Delta}$ associado a $\frac{1}{s} f$.

\section{A Métrica Produto}

Considere agora $\left(M_{1}, g^{(1)}\right)$ uma variedade riemanniana compacta (sem bordo) e $\left(M_{2}, \bar{g}^{(2)}\right)$ uma variedade riemanniana compacta com bordo $\partial M_{2} \neq \emptyset$. Sejam $m_{1}$ e $m_{2}$ as dimensões de $M_{1}$ e $M_{2}$, respectivamente, de modo que $m=m_{1}+m_{2} \geq 3$. Seja $M=M_{1} \times M_{2}$ a variedade produto, de dimensão $m$, com bordo $\partial M=M_{1} \times \partial M_{2}$ e métrica produto $g^{(1)} \oplus \bar{g}^{(2)}$ dada por

$$
g_{(p, q)}(X, Y)=g_{p}^{(1)}\left(X_{1}, Y_{1}\right)+\bar{g}_{q}^{(2)}\left(X_{2}, Y_{2}\right)
$$

onde $X=\left(X_{1}, X_{2}\right), Y=\left(Y_{1}, Y_{2}\right), X_{t}, Y_{t} \in \Gamma\left(T M_{t}\right), t=1,2 \mathrm{e}$

$$
\left(g_{i j}\right)_{i j}=\left(\begin{array}{c|c}
\left(g_{k l}^{(1)}\right)_{k l} & 0 \\
\hline 0 & \left(\bar{g}_{r s}^{(2)}\right)_{r s}
\end{array}\right)
$$

onde $i, j=1, \ldots, m, k, l=1, \ldots, m_{1}$ e $r, s=1, \ldots, m_{2}$. Se $\pi_{1}: M \longrightarrow M_{1}$ e $\pi_{2}: M \longrightarrow M_{2}$ são as projeções canônicas, a forma volume com respeito à métrica produto é dada por

$$
\omega_{g}=\pi_{1}^{*} \omega^{(1)} \cdot \pi_{2}^{*} \omega^{(2)}
$$

é fácil ver que

$$
\omega_{g}=\sqrt{\left|\operatorname{det}\left(g_{i j}\right)_{i j}\right|} d x^{1} \wedge \ldots \wedge d x^{m}=\sqrt{\left|\operatorname{det}\left(g_{k l}^{(1)}\right)_{k l}\right| \cdot\left|\operatorname{det}\left(\bar{g}_{r s}^{(2)}\right)_{r s}\right|} d x^{1} \wedge \ldots \wedge d x^{m} .
$$

Observe que estamos identificando o espaço tangente da variedade produto $T_{(p, q)} M$ com a soma direta $T_{p} M_{1} \oplus T_{q} M_{2}$. A conexão riemanniana com respeito à métrica produto é dada por

$$
\nabla_{X_{1}+X_{2}} Y_{1}+Y_{2}=\nabla_{X_{1}}^{(1)} Y_{1}+\nabla_{X_{2}}^{(2)} Y_{2}, \quad X_{i}, Y_{i} \in \Gamma\left(T M_{i}\right)
$$

e satisfaz

$$
\nabla_{X_{1}} Y_{1}=\nabla_{X_{1}}^{(1)} Y_{1}, \quad \nabla_{X_{2}} Y_{2}=\nabla_{X_{2}}^{(2)} Y_{2}, \quad \nabla_{X_{1}} X_{2}=\nabla_{X^{2}} X_{1}=0
$$

Agora que vimos que a conexão riemanniana da variedade produto é aditiva, podemos observar facilmente que o tensor de Riemann com respeito à métrica produto, é dado por

$$
R(X, Y) Z=R^{(1)}\left(X_{1}, Y_{1}\right) Z_{1}+R^{(2)}\left(X_{2}, Y_{2}\right)
$$

isto é, $R(X, Y) Z=\left(R^{(1)}\left(X_{1}, Y_{1}\right) Z_{1}, R^{(2)}\left(X_{2}, Y_{2}\right)\right)$. De fato, como

$$
R(X, Y) Z=\nabla_{X} \nabla_{Y} Z-\nabla_{Y} \nabla_{X} Z-\nabla_{[X, Y]} Z
$$


basta notar que

$$
\nabla_{X_{1}+X_{2}}\left(\nabla_{Y_{1}}^{(1)} Z_{1}+\nabla_{Y_{2}}^{(2)} Z_{2}\right)=\nabla_{X_{1}}^{(1)} \nabla_{Y_{1}}^{(1)} Z_{1}+\nabla_{X_{2}}^{(2)} \nabla_{Y_{2}}^{(2)} Z_{2}
$$

e

$$
[X, Y]=\nabla_{X} Y-\nabla_{Y} X=\nabla_{X_{1}}^{(1)} Y_{1}+\nabla_{X_{2}}^{(2)} Y_{2}-\nabla_{Y_{1}}^{(1)} X_{1}-\nabla_{Y_{2}}^{(2)} X_{2}=\left[X_{1}, Y_{1}\right]+\left[X_{2}, Y_{2}\right]
$$

Decorre diretamente que

$$
R i c_{g}=R i c_{g^{(1)}}+R i c_{\bar{g}^{(2)}}
$$

e

$$
R_{g}=R_{g^{(1)}}+R_{\bar{g}^{(2)}}
$$

Para $p \in M_{1}$ e $q \in M_{2}$, o campo vetorial interior $\eta_{g}$, normal a $\partial M$, pode ser escrito como

$$
\eta_{g}=0+\eta_{2}
$$

onde $\eta_{2}$ é o campo vetorial interior normal a $\partial M_{2}$. Logo, a segunda forma fundamental e a curvatura média de $\partial M$ são dadas, respectivamente por

$$
I I_{g}(X, Y)=I I_{\bar{g}^{(2)}}\left(X_{2}, Y_{2}\right) \quad \text { e } \quad H_{g}=H_{\bar{g}^{(2)}}
$$

Combinando os resultados apresentados acima, para a métrica produto, com os resultados apresentados anteriormente, para homotetias da métrica, encontra-se facilmente os resultados utilizados na Seção 2.3.3.

\section{Operador Laplaciano}

O laplaciano em uma variedade riemanniana $(M, g)$ é o operador

$$
\Delta_{g}: \mathcal{C}^{k}(M) \longrightarrow \mathcal{C}^{k-2}(M)
$$

onde $k \geq 2$, definido por

$$
\Delta_{g} f=-\operatorname{div}_{g} \nabla_{g} f,
$$

ou seja, o laplaciano é o divergente do campo gradiente de uma função $f \in \mathcal{C}^{k}(M)$. Para toda $\varphi, \psi \in \mathcal{C}^{k}(M), \alpha \in \mathbb{R}$, o laplaciano é um operador linear

$$
\Delta_{g}(\alpha \varphi+\psi)=\alpha \Delta_{g} \varphi+\Delta_{g} \psi
$$

e satisfaz

$$
\Delta_{g}(\varphi \cdot \psi)=\varphi \Delta_{g} \psi+\psi \Delta_{g} \varphi-2 g(\nabla \varphi, \nabla \psi)
$$

Se $M$ é uma variedade riemanniana compacta com bordo mínimo, o operador laplaciano é simétrico, com respeito ao produto $L^{2}(M)$, no espaço das funções suaves, definidas em $M$, que satisfazem a 
condição de Neumann, $\partial_{\eta}(\cdot)=0$,

$$
\begin{aligned}
\left\langle\Delta_{g} \psi, \varphi\right\rangle & =\int_{M}\left(\Delta_{g} \psi\right) \varphi \omega_{g}=\int_{M}-\operatorname{div}_{g}\left(\nabla_{g} \psi\right) \varphi \omega_{g} \\
& =\int_{M}\left\langle\nabla_{g} \psi, \nabla_{g} \varphi\right\rangle \omega_{g}-\int_{M} \operatorname{div}_{g}\left[\left(\nabla_{g} \psi\right) \varphi\right] \omega_{g} \\
& =\int_{M}\left\langle\nabla_{g} \psi, \nabla_{g} \varphi\right\rangle \omega_{g}+\int_{\partial M}\left\langle\left(\nabla_{g} \psi\right) \varphi, \eta_{g}\right\rangle \sigma_{g} \\
& =\int_{M} \operatorname{div}_{g}\left[\psi\left(\nabla_{g} \varphi\right)\right] \omega_{g}-\int_{M} \psi \operatorname{div}_{g}\left(\nabla_{g} \varphi\right) \omega_{g}+\int_{\partial M} \varphi \partial \phi \sigma_{g} \\
& =-\int_{\partial M} \psi\left\langle\left(\nabla_{g} \varphi\right), \eta_{g}\right\rangle \sigma_{g}+\int_{M} \psi\left(\Delta_{g} \varphi\right) \omega_{g} \\
& =-\int_{\partial M} \psi \partial_{\eta} \varphi \sigma_{g}+\int_{M} \psi\left(\Delta_{g} \varphi\right) \omega_{g} \\
& =\int_{M} \psi \Delta_{g} \varphi \omega_{g} \\
& =\left\langle\psi, \Delta_{g} \varphi\right\rangle .
\end{aligned}
$$

As mesmas contas feitas acima mostram que o laplaciano é simétrico também no espaço das funções satisfazendo a condição de Dirichlet (isto é, quando se anulam no bordo). Ainda, quando $\partial M=\emptyset$, o operador $\Delta$ é simétrico no espaço das funções (suficientemente regulares) em $M$.

Além disso, existem alguns fatos gerais ([3], pag. 53), bem conhecidos, sobre o espectro do laplaciano em uma variedade compacta, que passamos a exibir abaixo.

Teorema 1.5.3. Seja $(M, g)$ uma variedade compacta com bordo $\partial M$, e considere o problema de autovalor $\Delta_{g} \phi=\lambda \phi$ com $\partial M \neq \emptyset$, com condições de Neumann ou com condições de Dirichlet no bordo. Então:

(i.) o conjunto de autovalores consiste de uma sequência infinita

$$
0 \leq \lambda_{0}<\lambda_{1}<\ldots<\lambda_{k}<\ldots,
$$

onde $\lambda_{0}=0$ não é um autovalor do problema de Dirichlet;

(ii.) cada autovalor tem multiplicidade finita e os autoespaços correspondentes a autovalores distintos são $L^{2}(M)$-ortogonais;

(iii.) a soma direta dos autoespaços $V_{\lambda_{i}}$ é um subconjunto denso em $L^{2}(M)$ com a norma $L^{2}$. Além disso, cada autofunção é suave.

Agora, vamos considerar a variedade produto $M=M_{1} \times M_{2}$, já definida, com a métrica $g=g^{(1)} \oplus \bar{g}^{(2)}$. Segue das observações acima que existem bases ortonormais, $\left\{f_{\alpha}^{(1)}\right\}_{\alpha}$ de $L^{2}\left(M_{1}\right)$ e $\left\{f_{\beta}^{(2)}\right\}_{\beta}$ de $L^{2}\left(M_{2}\right)$, formadas por autofunções de $\Delta_{g^{(1)}}$ e de $\Delta_{\bar{g}^{(2)}}$, respectivamente. Afirmamos que $\left\{f_{\alpha}^{(1)} \otimes f_{\beta}^{(2)}\right\}_{\alpha, \beta}$ é uma base ortonormal de autofunções de $\Delta_{g}$ para $L^{2}(M)$. De fato, sejam $f^{(1)}, f^{(2)}$ autofunções de $\Delta_{g^{(1)}}, \Delta_{\bar{g}^{(2)}}$, associadas aos autovalores $\rho^{(1)}, \rho^{(2)}$, respectivamente. Se $\pi_{1}: M \longrightarrow M_{1}$ e $\pi_{2}: M \longrightarrow M_{2}$ são as projeções canônicas, temos

$$
\begin{aligned}
\Delta_{g}\left(f^{(1)} \otimes f^{(2)}\right) & =\left(\Delta_{g} f^{(1)}\right) \otimes f^{(2)}+f^{(1)} \otimes \Delta_{g} f^{(2)}-2 g\left(\nabla_{g} f^{(1)}, \nabla_{g} f^{(2)}\right) \\
& =\left(\Delta_{g^{(1)}} f^{(1)}\right) \otimes f^{(2)}+f^{(1)} \otimes \Delta_{\bar{g}^{(2)}} f^{(2)} \\
& =\rho^{(1)} f^{(1)} \otimes f^{(2)}+f^{(1)} \otimes \rho^{(2)} f^{(2)} \\
& =\left(\rho^{(1)}+\rho^{(2)}\right) f^{(1)} \otimes f^{(2)},
\end{aligned}
$$


isto é, $f^{(1)} \otimes f^{(2)}$ é autofunção de $\Delta_{g}$ associada ao autovalor $\rho^{(1)}+\rho^{(2)}$. Além disso, para todo $k \in \mathbb{R}$, o conjunto

$$
\left\{\sum_{\alpha, \beta} k\left(f_{\alpha}^{(1)} \otimes f_{\beta}^{(2)}\right)\right\}
$$

é denso em $L^{2}(M)$ e $f_{\alpha}^{(1)} \otimes f_{\beta}^{(2)}(x, y)=f_{\alpha}^{(1)}(x) \cdot f_{\beta}^{(2)}(y)$, para todo $x \in M_{1}, y \in M_{2}$. Como

$$
\Delta_{g}\left(f^{(1)} \otimes f^{(2)}\right)=\left(\Delta_{g^{(1)}} f^{(1)}\right) \otimes f^{(2)}+f^{(1)} \otimes \Delta_{\bar{g}^{(2)}} f^{(2)},
$$

para toda $f^{(1)} \in L^{2}\left(M_{1}\right), f^{(2)} \in L_{2}\left(M_{2}\right)$, escrevemos

$$
\Delta_{g}=\Delta_{g^{(1)}} \otimes I_{2}+I_{1} \otimes \Delta_{\bar{g}^{(2)}}
$$

onde $I_{i}$ é o operador identidade em $L^{2}\left(M_{i}\right)$.

Mais que isso, é importante ver que todos os autovalores do laplaciano $\Delta_{g}$ são da forma $\rho^{(1)}+$ $\rho^{(2)}$. Com efeito, suponha que $f \neq 0$ seja autofunção de $\Delta_{g}$ associada ao autovalor $\lambda$. Considere o produto interno canônico no espaço $L^{2}(M)$, então para todo elemento $f_{\alpha}^{(1)} \otimes f_{\beta}^{(2)}$ da base de autofunções exibida, temos

$$
\begin{aligned}
\lambda\left\langle f, f_{\alpha}^{(1)} \otimes f_{\beta}^{(2)}\right\rangle & =\left\langle\lambda f, f_{\alpha}^{(1)} \otimes f_{\beta}^{(2)}\right\rangle \\
& =\left\langle\Delta_{g} f, f_{\alpha}^{(1)} \otimes f_{\beta}^{(2)}\right\rangle \\
& =\left\langle f, \Delta_{g}\left(f_{\alpha}^{(1)} \otimes f_{\beta}^{(2)}\right)\right\rangle \\
& =\left\langle f,\left(\rho_{\alpha}^{(1)}+\rho_{\beta}^{(2)}\right) f_{\alpha}^{(1)} \otimes f_{\beta}^{(2)}\right\rangle \\
& =\rho_{\alpha}^{(1)}+\rho_{\beta}^{(2)}\left\langle f, f_{\alpha}^{(1)} \otimes f_{\beta}^{(2)}\right\rangle
\end{aligned}
$$

faça $\rho_{\alpha, \beta}=\rho_{\alpha}^{(1)}+\rho_{\beta}^{(2)}$, então

$$
\lambda-\rho_{\alpha, \beta}\left\langle f, f_{\alpha}^{(1)} \otimes f_{\beta}^{(2)}\right\rangle=0,
$$

para todo $\alpha, \beta$, isto é, se supormos $\lambda \neq \rho_{\alpha, \beta}$, então $f$ é ortogonal a todos os elementos de $\left\{\sum_{\alpha, \beta} k\left(f_{\alpha}^{(1)} \otimes f_{\beta}^{(2)}\right)\right\}$, mas o único elemento ortogonal a um subespaço denso é o zero, donde $f=0$, o que é uma contradição. 


\section{Capítulo 2}

\section{Análise Funcional}

Um espaço normado é um espaço vetorial com uma norma nele definida. Um espaço de Banach é um espaço vetorial normado, completo com respeito à métrica induzida pela norma

$$
d(x, y)=\|x-y\| .
$$

Proposição 2.0.4. Um subespaço $Y$ de um espaço de Banach $X$ é completo se e somente se o conjunto $Y$ é fechado em $X$.

Segue do resultado acima que todo subespaço fechado de um espaço de Banach é um espaço de Banach.

Definição 2.0.5. Uma norma $\|\cdot\|_{1}$ em um espaço vetorial $X$ é dita equivalente a uma norma $\|\cdot\|_{2}$ se existem constantes positivas $c_{1}$ e $c_{2}$ tais que

$$
c_{1}\|x\|_{1} \leq\|x\|_{2} \leq c_{2}\|x\|_{1}
$$

para todo $x \in X$.

Esta definição é importante, pois normas equivalentes definem a mesma topologia em $X$.

Definição 2.0.6. Sejam $X, Y$ espaços normados. Dizemos que o operador linear $T: X \longrightarrow Y$ é limitado, se existe um número real c tal que, para todo $x \in X$,

$$
\|T x\|_{Y} \leq c\|x\|_{X}
$$

A norma de $T$ é definida por

$$
\|T\|=\inf \{c \in \mathbb{R}:\|T x\| \leq c\|x\|, \forall x \in X\},
$$

equivalentemente,

$$
\|T\|=\sup _{x \neq 0} \frac{\|T x\|}{\|x\|} \text { ou }\|T\|=\sup _{\|x\|=1}\|T x\| .
$$

A composição de operadores limitados é um operador limitado e

$$
\|T \circ S\| \leq\|T\| \cdot\|S\| \text {. }
$$

Proposição 2.0.7. Um operador linear $T: X \longrightarrow Y$ entre espaços normados é limitado se e somente se é continuo.

Demonstração. Para $T=0$ a afirmação é válida. Se $T \neq 0$, então $\|T\| \neq 0$. Seja $x_{0} \in X$. Dado 
$\varepsilon>0$, sempre que $\left\|x-x_{0}\right\|<\delta, \operatorname{com} \delta=\frac{\varepsilon}{\|T\|}$, temos

$$
\left\|T x-T x_{0}\right\|=\left\|T\left(x-x_{0}\right)\right\| \leq\|T\|\left\|x-x_{0}\right\|<\|T\| \delta=\varepsilon,
$$

donde $T$ é contínuo. Reciprocamente, se $T$ é contínuo em $x_{0}$ (arbitrário), dado $\varepsilon>0$, existe $\delta>0$ tal que $\left\|T x-T x_{0}\right\| \leq \varepsilon$ para todo $x \in X$ que satisfaça $\left\|x-x_{0}\right\| \leq \delta$, em particular para

$$
x=x_{0}+\frac{\delta}{\|y\|} y,
$$

para qualquer $0 \neq y \in X$, segue que

$$
\varepsilon \geq\left\|T x-T x_{o}\right\|-\left\|T\left(x-x_{0}\right)\right\|=\left\|T\left(\frac{\delta}{\|y\|} y\right)\right\|=\frac{\delta}{\|y\|}\|T y\|,
$$

portanto, $\|T y\| \leq \frac{\varepsilon}{\delta}\|y\|$, o que implica que $T$ é limitado.

Segue como corolário que se $T$ é limitado, $\operatorname{ker} T$ é fechado.

O espaço vetorial $B(X, Y)$ de todos os operadores limitados de um espaço normado $X$ em um espaço normado $Y$ é um espaço normado com a norma de operadores. Se $Y$ é um espaço de Banach, então $B(X, Y)$ é um espaço de Banach.

Um espaço com produto interno é um espaço vetorial com um produto interno nele definido. Um espaço de Hilbert é um espaço com produto interno, completo com respeito à norma definida por este produto interno.

Lema 2.0.8. Seja $X \neq \emptyset$ um subconjunto de um espaço de Hilbert $H$. O espaço gerado por $X$ é denso em $H$ se e somente se $X^{\perp}=\{0\}$.

Definição 2.0.9. Se existem $T: X \longrightarrow Y$ e $T^{-1}: Y \longrightarrow X$ limitadas então $X$ e $Y$ são ditos topologicamente isomorfos.

Proposição 2.0.10. Seja $T: E \longrightarrow F$ um operador linear limitado entre espaços de Banach. Se $T$ admite um inverso contínuo à direita $S: F \longrightarrow E$, então, o núcleo de $T, \operatorname{ker} T$, admite um complemento fechado; esse complemento é a imagem de $S$.

Demonstração. Seja $S: F \longrightarrow E$ um inverso contínuo à direita de $T$. Vamos mostrar que $E=\operatorname{ker} T \oplus \operatorname{Im} S$. Seja $x \in E$, então $x-S \circ T(x) \in \operatorname{ker} T$ :

$$
T(x-S \circ T(x))=T(x)-T(S \circ T(x))=T(x)-\underbrace{T \circ S}_{\text {id }}(T(x))=0 .
$$

Então, podemos escrever

$$
x=(x-S \circ T(x))+S(T(x)),
$$

isto é, $E=\operatorname{ker} T+\operatorname{Im} S$. Agora, suponha que $x \in \operatorname{ker} T \cap \operatorname{Im} S$, então $x=S(y)$ para algum $y \in F$ e $0=T(x)=T(S(y))=y$, donde $x=S(0)=0$. Portanto, $E=\operatorname{ker} T \oplus \operatorname{Im} S$. Vejamos agora que $\operatorname{Im} S$ é fechado. Seja $\left(y_{n}\right)_{n>0}$ uma sequência de pontos em $F$ tal que $S\left(y_{n}\right) \rightarrow x$, para algum $x \in E$. Pela continuidade de $T$, segue que $T \circ S\left(y_{n}\right) \rightarrow T(x)$, isto é, $y_{n} \rightarrow T(x)$. Como $S$ é contínuo, temos $S\left(y_{n}\right) \rightarrow S(T(x))$. Pela unicidade do limite, $x=S(T(x))$, ou seja $x \in \operatorname{Im} S$.

Claramente, o resultado simétrico ao que acabamos de provar é válido, isto é, se $S$ tem inverso contínuo $T$ à esquerda, então ImS admite complemento fechado em $E$ (que é o núcleo de T). Além disso, se supusermos, no enunciado da proposição acima, que $T$ é sobrejetor, vale a recíproca.

Proposição 2.0.11. Se $T: E \longrightarrow F$ é um operador linear limitado de um espaço de Banach $E$ sobre um espaço de Banach $F$ e o núcleo de $T$ tem complemento fechado em E, então $T$ admite inverso contínuo à direita. 
Demonstração. Seja $W$ um subespaço fechado de $E$, tal que $W \oplus \operatorname{ker} T=E$. Como $T$ é sobrejetivo, para cada $y \in F$, existe $x=w+k \in E$, com $w \in W$ e $k \in \operatorname{ker} T$, tal que $T(x)=y$. Mas, $y=T(x)=T(w+k)=T(w)+T(k)=T(w)$. Assim, para cada $y \in F$, defina $S: F \rightarrow E$ por

$$
S(y):=w,
$$

isto é, $S(y)=P_{W}(x)$, onde $P_{W}$ é a projeção de $x$ sobre $W$.

(1) $S$ está bem definida. De fato, seja $y=y_{1}=y_{2} \in F$, então existem $x_{1}=w_{1}+k_{1}, x_{2}=$ $w_{2}+k_{2} \in E$ tais que $T\left(x_{1}\right)=y_{1}=y_{2}=T\left(x_{2}\right)$. Como $T$ é sobrejetor, existe um isomorfismo $\theta: E / \operatorname{ker} T \longrightarrow F$ tal que $\theta \circ \pi=T$, onde $\pi$ é a projeção canônica no espaço quociente; assim, temos $T\left(x_{1}\right)=\theta\left(\pi\left(x_{1}\right)\right)=\theta\left(\overline{x_{1}}\right)=\theta\left(\overline{w_{1}+k_{1}}\right)$ e $T\left(x_{2}\right)=\theta\left(\pi\left(x_{2}\right)\right)=\theta\left(\overline{x_{2}}\right)=\theta\left(\overline{w_{2}+k_{2}}\right)$. Por outro lado, existe uma única classe de equivalência $\bar{x} \in E / \operatorname{ker} T$ que $\theta$ faz corresponder à $y$, isto é, $\bar{x}=\overline{x_{1}}=\overline{x_{2}}$. Note que, como $k_{1}, k_{2} \in \operatorname{ker} T$, segue que $\overline{x_{1}}=\overline{w_{1}}$ e $\overline{x_{2}}=\overline{w_{2}}$ e como $w_{1}$ e $w_{2}$ estão no complementar de $\operatorname{ker} T$, temos que $\overline{x_{1}}=\overline{x_{2}} \Leftrightarrow w_{1}=w_{2}$. Portanto, mesmo que $x_{1} \neq x_{2}$, mostramos que, se $T\left(x_{1}\right)=T\left(x_{2}\right)=y$ segue que $w_{1}=w_{2}=w$, de modo que fica bem definido $S(y)=w$.

(2) $S$ é linear. Sejam $\alpha$ um escalar e $y_{1}, y_{2} \in F$, então $S\left(\alpha y_{1}+y_{2}\right)=w$, onde $w=x-k$ com $T(x)=\alpha y_{1}+y_{2}$. Como $T$ é sobrejetor, existem $x_{1}=w_{1}+k_{1}$ e $x_{2}=w_{2}+k_{2}$ em $E$ tais que $T\left(x_{1}\right)=y_{1}$ e $T\left(x_{2}\right)=y_{2}$, ou seja, $S\left(y_{1}\right)=w_{1}$ e $S\left(y_{2}\right)=w_{2}$. Além disso,

$$
\alpha x_{1}+x_{2}=\underbrace{\alpha w_{1}+w_{2}}_{\in W}+\underbrace{\alpha k_{1}+k_{2}}_{\in \operatorname{ker} T}
$$

e, pela linearidade de $T$, temos também $T(x)=\alpha T\left(x_{1}\right)+T\left(x_{2}\right)=T\left(\alpha x_{1}+x_{2}\right)$. Por definição, temos $S\left(\alpha y_{1}+y_{2}\right)=\alpha w_{1}+w_{2}$, pelo item anterior, devemos ter necessariamente $w=\alpha w_{1}+w_{2}$, isto é, $S\left(\alpha y_{1}+y_{2}\right)=\alpha S\left(y_{1}\right)+S\left(y_{2}\right)$.

(3) $S$ é inverso à direita de $T$. Se $x=w+k$ e $T(x)=y$, então $S(y)=w$ e $T(w)=y$, já que $k$ está no núcleo de $T$ e $T$ é linear. Como $T$ é sobrejetivo, para todo $y \in F$, temos $T \circ S(y)=T(w)=y$.

(4) $S$ é contínuo. Basta notar que $\left(\left.T\right|_{W}\right)$ é um isomorfismo linear limitado, portanto $\left(\left.T\right|_{W}\right)^{-1}$ é limitado de $F$ em $W$. Como $S=\left(\left.T\right|_{W}\right)^{-1}, S: F \longrightarrow E$ é limitado, portanto, contínuo.

Definição 2.0.12. Sejam $X$ e $Y$ espaços normados. Um operador $T: X \longrightarrow Y$ é dito compacto se a imagem de qualquer conjunto limitado de $X$, por $T$, tem fecho compacto em $Y$.

Todo operador compacto é limitado. Se a dimensão de $X$ é infinita, o operador identidade de $X$ não é compacto, em geral. Além disso, se $T$ e $S$ são operadores compactos entre os espaços normados $X$ e $Y$, e $R$ é um operador limitado de $Y$ em um espaço normado $Z$, então $R \circ T$ e $\alpha T+\beta S$ são operadores compactos, para todo $\alpha, \beta \in \mathbb{R}$.

\section{Variedades de Banach}

Unindo a noção de variedade ao conceito de espaços de Banach, podemos definir uma variedade de Banach como um espaço topológico modelado em um espaço de Banach. É um meio de obtermos variedades de dimensão infinita, usando espaços de Banach de dimensão infinita no lugar do $\mathbb{R}^{n}$.

Definição 2.0.13. Seja $M$ um espaço topológico e $E$ um espaço de Banach. Um atlas $\mathcal{C}^{k}$ para $M$ sobre E é uma família de pares $\left\{\left(U_{\alpha}, \phi_{\alpha}\right)\right\}_{\alpha}$ com as seguintes propriedades:

a. $\left\{U_{\alpha}\right\}_{\alpha}$ é uma cobertura aberta para $M$;

b. $\phi_{\alpha}$ é um homeomorfismo de $U_{\alpha}$ sobre um subconjunto aberto de E;

c. para cada $\alpha, \beta$ tais que $U_{\alpha} \cap U_{\beta} \neq \emptyset$, a aplicação

$$
\phi_{\beta} \circ \phi_{\alpha}^{-1}: \phi_{\alpha}\left(U_{\alpha} \cap U_{\beta}\right) \longrightarrow \phi_{\beta}\left(U_{\alpha} \cap U_{\beta}\right)
$$


é um difeomorfismo de classe $\mathcal{C}^{k}$.

Um espaço topológico $M$ com um atlas $\mathcal{C}^{k}$ sobre $E$ é chamado de variedade de Banach de classe $\mathcal{C}^{k}$.

Seja $f: N \longrightarrow M$ uma aplicação diferenciável entre variedades diferenciáveis. Vimos que, se $f$ é uma imersão injetora e a topologia herdada de $N$ por $f(N)$ coincide com a topologia induzida em $f(N)$ por $M$, então $f(N) \subset M$ é uma subvariedade (regular) de $M$. Também vimos que se $p \in M$ é um valor regular de $f$, então $P=f^{-1}(p) \subset N$ é uma subvariedade (regular) de $N$. Se tomarmos a inclusão, no primeiro caso, teremos $i_{M}: f(N) \hookrightarrow M$ e $\operatorname{Im}\left(\mathrm{d} i_{M}\right)_{p}=T_{p} f(N)$. Em dimensão finita, é claro que $T_{p} f(N)$ tem complemento em $M$, isto é, existe um subespaço $S$ de $T_{p} M$ tal que $T_{p} f(N)+S=T_{p} M$. No segundo caso, $i_{N}: P \hookrightarrow N$ e $\operatorname{Im}\left(\mathrm{d} i_{N}\right)_{q}=T_{q} P=\operatorname{ker}(\mathrm{d} f)_{q}$, também, em dimensão finita, $T_{q} P$ é complementado em $N$. Vejamos o que ocorre em dimensão infinita, onde não podemos garantir a complementação do espaço tangente de forma automática.

Definição 2.0.14. Seja $M$ uma variedade (diferenciável) de Banach. Um subconjunto $N \subset M$ é uma subvariedade de Banach de $M$, se para todo $p \in N$, existe uma carta $(U, \phi)$ de $M$, com $p \in U$, tal que

(i.) o subconjunto $X=\phi(U)$, do espaço de Banach $E$ (modelo para $M)$, contém um subespaço fechado $Y$ que é complementado em $X$;

(ii.) $\phi(U \cap N)$ é aberto em $Y$.

Na definição acima, observe que $X$ é homeomorfo a $T_{p} M$. Seja $\tilde{Y} \subset T_{p} M$ o subespaço correspondente a $Y \subset X$. Então, se $N$ é subvariedade de Banach de $M$, segue da definição que $\widetilde{Y}$ tem complemento fechado tem $T_{p} M$. Além disso, $\tilde{Y}=T_{p} N$.

\section{$2.1 \quad$ Operadores de Fredholm}

Sejam $B_{1}$ e $B_{2}$ espaços de Banach.

Definição 2.1.1. Seja $T: B_{1} \longrightarrow B_{2}$ um operador linear limitado. Dizemos que $T$ é de Fredholm se

(i.) o núcleo, $\operatorname{ker} T$, tem dimensão finita,

(ii.) a imagem, ImT, é um subespaço fechado,

(iii.) o co-núcleo, cokerT, tem dimensão finita.

O co-núcleo de $T$ é definido pelo quociente de $B_{2}$ pela imagem de $T$, portanto a dimensão do co-núcleo de $T$ coincide com a codimensão da imagem de $T$. Se $T$ é de Fredholm definimos e denotamos o indice de $T$ por

ind $T:=\operatorname{dim} \operatorname{ker} T-\operatorname{codim} \operatorname{Im} T$.

O lema abaixo, mostra que o item (ii.) da definição acima é redundante.

Lema 2.1.2. Seja $T: B_{1} \longrightarrow B_{2}$ um operador linear limitado. Se a imagem de $T$ tem codimensão finita, então Im Té fechado.

Demonstração. Primeiro, suponha que $\operatorname{ker} T=\{0\}$. Seja $V$ um complemento de $\operatorname{Im} T$ em $B_{2}$. Como a dimensão de $V$ é finita, $V$ é um espaço de Banach com a norma herdada de $B_{2}$, bem como $B_{1} \times V$. Agora, considere a aplicação $S: B_{1} \times V \longrightarrow B_{2}$ dada por

$$
S(x, v)=T(x)+v,
$$


então $S$ é uma bijeção, linear, contínua. Pelo Teorema da Aplicação Aberta, $S$ é um homeomorfismo, portanto, $\operatorname{Im} T=S\left(B_{1} \times\{0\}\right)$ é fechado em $B_{2}$. No caso geral, note que $B_{1} / \operatorname{ker} T$ é um espaço de Banach e temos $T=\widetilde{T} \circ \pi$, onde $\pi: B_{1} \longrightarrow B_{1} / \operatorname{ker} T$ e $\widetilde{T}$ é a aplicação induzida no quociente. Como $\widetilde{T}$ é injetora, podemos aplicar a primeira parte da demonstração a $\widetilde{T}$, concluindo que $\operatorname{Im} T=\operatorname{Im} \widetilde{T}$ é fechado.

Observe que, se $T$ é de Fredholm de índice zero, $T$ é injetivo se e somente se $T$ é sobrejetivo.

Proposição 2.1.3. Sejam $T: B_{1} \longrightarrow B, S: B \longrightarrow B_{2}$ operadores de Fredholm e $K: B_{1} \longrightarrow B$ um operador compacto. Então,

(i.) se $\varepsilon: B_{1} \longrightarrow B$ é um operador limitado com $\|\varepsilon\|$ suficientemente pequena, $T+\varepsilon$ é um operador de Fredholm e ind $(T+\varepsilon)=$ ind $T$,

(ii.) $T+K$ é de Fredholm e ind $(T+K)=$ ind $T$,

(iii.) $S \circ T$ é de Fredholm e ind $(S \circ T)=$ ind $S+$ ind $T$.

Os itens (i.) e (ii.) da proposição acima, nos mostra que operadores de Fredholm são invariantes quando submetidos a pequenas perturbações ou sob perturbações compactas.

\subsection{Espaços de Hölder}

Seja $k \geq 1$. Um elemento do conjunto

$$
\left.\mathcal{A}_{k}=\left\{a=\left(a_{1}, \ldots a_{n}\right)\right\}: a_{i} \geq 0, \sum_{i=1}^{n} a_{i}=k\right\}
$$

é chamado multi-índice de ordem $k$.

Sejam $f: \mathbb{R}^{n} \longrightarrow \mathbb{R}$, com $f=f\left(x_{1}, \ldots, x_{n}\right)$, e $a \in \mathcal{A}_{k}$, então

$$
D^{a} f=D_{1}^{a_{1}} \cdot \ldots \cdot D_{n}^{a_{n}} f=\frac{\partial^{a_{1}}}{\partial x_{1}^{a_{1}}} \cdot \ldots \cdot \frac{\partial^{a_{n}}}{\partial x_{1}^{a_{n}}} f
$$

denotada também por

$$
\frac{\partial^{|a|}}{\partial_{x_{1}}^{a_{1}} \ldots \partial_{x_{n}}^{a_{n}}} f, \quad \text { onde }|a|=\sum_{i=1}^{n} a_{i}=k
$$

Defina

$$
\nabla^{k} f:=\left\{D^{a} f\right\}_{a \in \mathcal{A}_{k}},
$$

isto é, $\nabla^{k} f$ contém todas as derivadas parciais de $f$ (cuja soma das ordens é igual a $k$ ).

Exemplo 2.2.1. (a.) Se $k=2$, podemos ter

$$
\begin{aligned}
D^{a} f=\frac{\partial^{2}}{\partial x \partial y} f, & a=(1,1), \\
D^{a} f=\frac{\partial^{2}}{\partial x^{2}} f, & a=(2,0), \\
D^{a} f=\frac{\partial^{2}}{\partial y^{2}} f, & a=(0,2) .
\end{aligned}
$$


(b.) Se $k=4$, podemos ter

$$
\begin{aligned}
D^{a} f & =\frac{\partial^{4}}{\partial x_{1} \partial x_{2} \partial x_{3} \partial x_{4}} f, & & a=(1,1,1,1), \\
D^{a} f & = & \frac{\partial^{4}}{\partial x_{4}^{4}} f, & a=(0,0,0,4), \\
D^{a} f & = & \frac{\partial^{4}}{\partial x_{1}^{2} x_{3}^{2}} f, & a=(2,0,2,0) .
\end{aligned}
$$

Definição 2.2.2. Sejam $\Omega \subset \mathbb{R}^{n}$ aberto e $k \geq 0$ um inteiro.

(i.) $O$ conjunto de funções que têm todas as derivadas parciais $D^{a} f$, com a $\in \mathcal{A}_{m}, 0 \leq m \leq k$, contínuas será denotado por $\mathcal{C}^{k}(\Omega)$.

(ii.) $\mathcal{C}^{k}(\bar{\Omega})$ é o conjunto de funções cujas derivadas, até ordem $k$, podem ser estendidas continuamente para $\bar{\Omega}$ e é munido da norma

$$
\|f\|_{\mathcal{C}^{k}}=\max _{0 \leq|a| \leq k} \sup _{x \in \Omega}\left|D^{a} f(x)\right|
$$

para toda $f \in \mathcal{C}^{k}(\bar{\Omega})$.

(iii.) $\mathcal{C}_{0}^{k}(\Omega)=\mathcal{C}_{0}(\Omega) \cap \mathcal{C}^{k}(\Omega)$, com

$$
\mathcal{C}_{0}(\Omega)=\left\{f \in \mathcal{C}^{0}(\Omega): \text { supp } f \subset \Omega \text { é compacto }\right\},
$$

onde supp $f=\overline{\{x \in \Omega: f(x) \neq 0\}}$ é o suporte de $f$.

(iv) $\mathcal{C}^{\infty}(\Omega)=\bigcap_{k=0}^{\infty} \mathcal{C}^{k}(\Omega)$, analogamente, $\mathcal{C}^{\infty}(\bar{\Omega})=\bigcap_{k=0}^{\infty} \mathcal{C}^{k}(\bar{\Omega})$,

(v) $\mathcal{C}_{0}^{\infty}(\Omega)=\mathcal{C}^{\infty}(\Omega) \cap \mathcal{C}_{0}(\Omega)$.

Observação 2.2.3. O espaço $\mathcal{C}^{k}(\bar{\Omega})$ munido da norma

$$
\|f\|_{\mathcal{C}^{k}(\bar{\Omega})}=\max _{0 \leq|a| \leq k} \sup _{x \in \bar{\Omega}}\left|D^{a} f(x)\right|
$$

é um espaço de Banach.

Sejam $D \subset \mathbb{R}^{n}, f: D \longrightarrow \mathbb{R}$ e $0<\alpha \leq 1$. Defina

$$
[f]_{\mathcal{C}^{0, \alpha}(D)}:=\sup _{\substack{x, y \in D \\ x \neq y}}\left\{\frac{|f(x)-f(y)|}{|x-y|^{\alpha}}\right\} .
$$

Definição 2.2.4. Sejam $\Omega \subset \mathbb{R}^{n}$ um aberto, $k \geq 0$ um inteiro. Os espaços de Hölder de funções continuas são definidos por

(i.) $\mathcal{C}^{0, \alpha}(\Omega)=\left\{f \in \mathcal{C}^{0}(\Omega):[f]_{\mathcal{C}^{0, \alpha}(K)}<\infty, \forall K \subset \Omega\right.$ compacto. $\}$,

(ii.) $\mathcal{C}^{0, \alpha}(\bar{\Omega})=\left\{f \in \mathcal{C}^{0}(\bar{\Omega}):[f]_{\mathcal{C}^{0, \alpha}(\bar{\Omega})}<\infty\right\}$. Note que $[f]_{\mathcal{C}^{0, \alpha}(D)}=L<\infty$ implica em

$$
|f(x)-f(y)| \leq L|x-y|^{\alpha}, \quad \forall x, y \in D, 0<\alpha \leq 1 .
$$

A norma em $\mathcal{C}^{0, \alpha}(\bar{\Omega})$ é dada por

$$
\|f\|_{\mathcal{C}^{0, \alpha}(\bar{\Omega})}=\|f\|_{\mathcal{C}^{0}(\bar{\Omega})}+[f]_{\mathcal{C}^{0, \alpha}(\bar{\Omega})},
$$


(iii.) $\mathcal{C}^{k, \alpha}(\Omega)=\left\{f \in \mathcal{C}^{k}(\Omega):\left[D^{a} f\right]_{\mathcal{C}^{0, \alpha}(K)}<\infty, \forall K \subset \Omega\right.$ compacto, $\left.\forall a \in \mathcal{A}_{k} \cdot\right\}$,

(iv.) $\mathcal{C}^{k, \alpha}(\bar{\Omega})=\left\{f \in \mathcal{C}^{k}(\bar{\Omega}):\left[D^{a} f\right]_{\mathcal{C}^{0, \alpha}(\bar{\Omega})}<\infty, \forall a \in \mathcal{A}_{k}\right.$. $\}$ com a norma

$$
\|f\|_{\mathcal{C}^{k, \alpha}(\bar{\Omega})}=\|f\|_{\mathcal{C}^{k}(\bar{\Omega})}+\max _{a \in \mathcal{A}_{k}}\left[D^{a} f\right]_{\mathcal{C}^{0, \alpha}(\bar{\Omega})},
$$

é um espaço de Banach.

Observação 2.2.5. (a.) No item (ii.) da definição acima, lembre que

$$
\|f\|_{\mathcal{C}^{0}(\bar{\Omega})}=\sup _{x \in \bar{\Omega}}|f(x)| .
$$

(b.) Escrevemos, por convenção, $\mathcal{C}^{k}(\bar{\Omega})=\mathcal{C}^{k, 0}(\bar{\Omega})$.

(c.) Observe que $\mathcal{C}^{0,1}(\bar{\Omega})$ é o conjunto das funções lipschitzianas em $\bar{\Omega}$.

Decorre das definições que, se $\left[D^{a} f\right]_{\mathcal{C}^{0, \alpha}(\bar{\Omega})}=L<\infty$, temos

$$
\mathcal{C}^{k, \alpha}(\bar{\Omega})=\left\{f \in \mathcal{C}^{k}(\bar{\Omega}):\left|D^{a} f(x)-D^{a} f(y)\right| \leq L|x-y|^{\alpha}, \forall x, y \in \bar{\Omega}, x \neq y, \forall a \in \mathcal{A}_{k}\right\} .
$$

Proposição 2.2.6. Sejam $\Omega \subset \mathbb{R}^{n}$ aberto, $0 \leq \alpha \leq 1$ e $k \geq 0$ um inteiro. São válidas as seguintes propriedades:

(i.) Se $f, g \in \mathcal{C}^{0, \alpha}(\bar{\Omega})$, então $f \cdot g \in \mathcal{C}^{0, \alpha}(\bar{\Omega})$;

(ii.) $\operatorname{Se} 0 \leq \alpha \leq \beta \leq 1$, então $\mathcal{C}^{k}(\bar{\Omega}) \supset \mathcal{C}^{k, \alpha}(\bar{\Omega}) \supset \mathcal{C}^{k, \beta}(\bar{\Omega}) \supset \mathcal{C}^{k, 1}(\bar{\Omega})$;

(iii.) Se $\bar{\Omega}$ é limitado e convexo, então $\mathcal{C}^{k, 1}(\bar{\Omega}) \supset \mathcal{C}^{k+1}(\bar{\Omega})$. Obviamente, $\mathcal{C}^{k-1, \alpha}(\bar{\Omega}) \supset \mathcal{C}^{k, \alpha}(\bar{\Omega})$ vale sempre.

Demonstração. A demonstração pode ser encontrada em [13].

Proposição 2.2.7. Seja $M$ uma variedade compacta. O conjunto

$$
\mathcal{C}_{+}^{k, \alpha}(M)=\left\{\phi \in \mathcal{C}^{k, \alpha}(M): \phi>0\right\}
$$

é aberto em $\mathcal{C}^{k, \alpha}(M)$ com a topologia induzia pela norma

$$
\|\phi\|_{\mathcal{C}^{k, \alpha}(M)}=\|\phi\|_{\mathcal{C}^{k}(M)}+\max _{a \in \mathcal{A}_{k}}\left\|D^{a} \phi\right\|_{\mathcal{C}^{k, \alpha}(M)} .
$$

Demonstração. Como vimos,

$$
\|\phi\|_{\mathcal{C}^{k, \alpha}(M)}=\|\phi\|_{\mathcal{C}^{k}(M)}+\max _{a \in \mathcal{A}_{k}}\left[D^{a} f\right]_{\mathcal{C}^{0, \alpha}(M)},
$$

isto é,

$$
\|\phi\|_{\mathcal{C}^{k, \alpha}(M)}=\max _{|\gamma| \leq k} \sup _{p \in M}\left|D^{\gamma} \phi(p)\right|+\max _{|\gamma| \leq k} \sup _{\substack{p, q \in M \\ p \neq q}} \frac{\left|D^{\gamma} \phi(p)-D^{\gamma} \phi(q)\right|}{|p-q|^{\alpha}}
$$

Seja $\phi \in \mathcal{C}_{+}^{k, \alpha}(M)$. Como $M$ é compacta, $\phi$ atinge um valor mínimo $m>0$ para algum $p \in M$. Seja $B_{\frac{m}{2}}(\phi)$ a bola aberta centrada em $\phi$ de raio $\frac{m}{2}$. Afirmamos que $B_{\frac{m}{2}}(\phi) \subset \mathcal{C}_{+}^{k, \alpha}(M)$. De fato, seja $\psi \in B_{\frac{m}{2}}(\phi)$, então $\|\phi-\psi\|_{\mathcal{C}^{k, \alpha}(M)}<\frac{m}{2}$. Em particular, $\left|D^{0}(\phi-\psi)(p)\right|<\frac{m}{2}$, para todo $p \in M$, o que implica em

$$
\phi(p)-\psi(p)<\frac{m}{2} \Longrightarrow \psi(p)>\phi(p)-\frac{m}{2} \geq m-\frac{m}{2}=\frac{m}{2}>0, \forall p \in M
$$


Na prova do próximo resultado, usaremos o Teorema de Arzelá-Áscoli. Vamos recordar seu enunciado.

Teorema 2.2.8 (Arzelá-Áscoli). Seja $M$ uma variedade compacta; $F \in \mathcal{C}(M)$ é um conjunto relativamente compacto se e somente se $F$ é equicontínuo e limitado para cada $x \in M$.

Lembre que um conjunto $S \in \mathcal{C}^{0}(M)$ é dito equicontínuo se para todo $x \in M$ e para todo $\varepsilon>0$, existe uma vizinhança $U_{x}$ tal que para todo $y \in U_{x}$ e para todo $f \in S$, temos $|f(x)-f(y)|<\varepsilon$; também que $S$ é dito limitado pontualmente se para cada $x \in M, \sup \{|f(x)|: f \in S\}<\infty$.

Proposição 2.2.9. Seja $M$ uma variedade compacta. A inclusão $\mathcal{C}^{k, \alpha}(M) \stackrel{i}{\longrightarrow} \mathcal{C}^{k-1, \alpha}(M), e^{-}$ compacta.

Demonstração. Seja $f \in B_{1}^{k}(0) \subset \mathcal{C}^{k, \alpha}(M)$, onde $B_{1}^{k}(0)$ é a bola unitária centrada na origem, então

$$
\|f\|_{\mathcal{C}^{k, \alpha}} \leq 1 \Rightarrow\|f\|_{\mathcal{C}^{k}}+\max _{|\gamma| \leq k} \sup _{\substack{x, y \in M \\ x \neq y}} \frac{\left|D^{\gamma} f(x)-D^{\gamma} f(y)\right|}{|x-y|^{\alpha}} \leq 1 .
$$

É claro que $\|i(f)\|_{\mathcal{C}^{k-1, \alpha}}=\|f\|_{\mathcal{C}^{k-1, \alpha}} \leq 1$

Seja $h \in i\left(B_{1}^{k}(0)\right)$, então $h=i(f)$, para algum $f \in B_{1}^{k}(0)$, assim, $\|h\|_{\mathcal{C}^{k-1, \alpha}}=\|i(f)\|_{\mathcal{C}^{k-1, \alpha}} \leq 1$, isto é, $i\left(B_{1}^{k}(0)\right) \subset B_{1}^{k-1}(0) \subset \mathcal{C}^{k-1, \alpha}(M)$. Portanto, $i\left(B_{1}^{k}(0)\right)$ é limitado.

Agora, vamos mostrar que $i\left(B_{1}^{k}(0)\right)$ é um conjunto de funções equicontínuas. Para todo $h \in$ $i\left(B_{1}^{k}(0)\right)$, temos

$$
\|h\|_{\mathcal{C}^{k-1, \alpha}} \leq 1 \Rightarrow\|h\|_{\mathcal{C}^{k-1}}+\max _{|\gamma| \leq k} \sup _{\substack{x, y \in M \\ x \neq y}} \frac{\left|D^{\gamma} h(x)-D^{\gamma} h(y)\right|}{|x-y|^{\alpha}} \leq 1
$$

isto é,

$$
\max _{|\gamma| \leq k} \sup _{x \in M}\left|D^{\gamma} h(x)\right|+\max _{|\gamma| \leq k} \sup _{\substack{x, y \in M \\ x \neq y}} \frac{\left|D^{\gamma} h(x)-D^{\gamma} h(y)\right|}{|x-y|^{\alpha}} \leq 1
$$

em particular,

$$
\frac{\left|D^{0} h(x)-D^{0} h(y)\right|}{|x-y|^{\alpha}}=\frac{|h(x)-h(y)|}{|x-y|^{\alpha}} \leq 1
$$

ou seja, dado $\varepsilon>0$, tome $\delta=\varepsilon^{\frac{1}{\alpha}}$, então

$$
|x-y|<\delta \Rightarrow|h(x)-h(y)| \leq|x-y|^{\alpha}<\varepsilon .
$$

Pelo Teorema de Arzelá-Áscoli, $i\left(B_{1}^{k}(0)\right)$ é relativamente compacto, como $i\left(B_{1}^{k}(0)\right)$ é fechado, segue que é compacto. 


\section{Capítulo 3}

\section{Representações de Grupos de Lie}

Lembremos que uma ação à esquerda de um grupo $G$ em um conjunto $X$ é uma aplicação

$$
\mu: G \times X \longrightarrow X
$$

que satisfaz

$$
\mu(e, x)=x, \quad \text { e } \quad \mu(g, \mu(h, x))=\mu(g h, x),
$$

onde $e$ é o elemento neutro do grupo, $g, h \in G, x \in X$. Algumas vezes a ação $\mu(g, x)$ é denotada simplesmente por $g \cdot x$. A ação à direita é definida de forma análoga. Comumente, ação de grupos está relacionada ao estudo de simetrias de um espaço geométrico. A teoria de representações se dedica a estudar os efeitos da ação de um grupo $G$ em um espaço vetorial $V$.

Definição 3.0.10. Seja $G$ um grupo de Lie e $V$ um espaço vetorial de dimensão finita sobre $\mathbb{R}$. Uma representação de $G$ em $V$ é um homomorfismo de grupos de Lie, $\pi: G \longrightarrow$ Aut $(V)$, de $G$ no grupo de automorfismos de $V$, isto é, $\pi$ é diferenciável, satisfaz

$$
\pi(g \cdot h)=\pi(g) \cdot \pi(h),
$$

e $\pi(g)$ é um isomorfismo de $V$ em si mesmo. O par $(V, \pi)$ é chamado de representação de $G$ e $V$ é o espaço de representação. A dimensão de $V$ é chamada grau da representação.

É usual na literatura, dizer apenas que $V$ é uma representação de $G$. Note que, fixada uma base, o grupo de automorfismos de um espaço vetorial $V$, de dimensão finita igual a $n$, sobre os reais, é isomorfo ao grupo geral linear, $G L_{n}(\mathbb{R})$, das matrizes invertíveis, com entradas reais, de ordem igual à $n=\operatorname{dim} V$. Assim, denotamos por $G L(V)$ o grupo geral linear em $V$ e dizemos que $\pi: G \longrightarrow G L(V)$ é a representação matricial de $G$ em $V$.

Uma representação $(V, \pi)$ é dita fiel se $\pi$ é um homomorfismo injetor; $\pi$ é chamada de representação trivial se cada elemento do grupo age como a identidade.

Podemos também definir um anti-homomorfismo de grupos de Lie, $\rho$, de $G$ em Aut $(V)$, isto é, uma aplicação que satisfaz

$$
\rho(g \cdot h)=\rho(h) \cdot \rho(g),
$$

à qual chamaremos antirrepresentação de $G$ em $V$.

Observe que $\pi(g): V \longrightarrow V$ dá a $V$ uma estrutura de módulo sobre $G$; de fato há uma relação biunívoca entre as representações (antirrepresentações) de $G$ em $V$ e as ações contínuas à esquerda (à direita) de $G$ sobre $V$, enquanto grupo abeliano com respeito à soma,

$$
\begin{aligned}
G \times V & \longrightarrow V \\
(g, v) & \longmapsto g \cdot v
\end{aligned}
$$

tais que, para cada $g \in G$, a translação à esquerda (à direita) $L_{g}: v \mapsto g \cdot v\left(R_{g}: v \mapsto v \cdot g\right)$ é 
linear. Diremos que $V$ é um $G$-módulo (módulo- $G$ ) real.

Em termos da translação à esquerda, uma ação à esquerda de $G$ em $V$ determina um homomorfismo linear

$$
L: G \longrightarrow \operatorname{Aut}(V),
$$

com inversa $L_{g^{-1}}$, satisfazendo $L_{e}=i d_{V}$ e $L_{g} \circ L_{h}=L_{g h}$.

Definição 3.0.11. Dadas duas representações de $G,(U, \pi),(V, \rho)$, um morfismo de representações é uma transformação linear $T$ entre os espaços $U$ e $V$ que é $G$-equivariante, isto é, para todo $g \in G$, $u \in U, T$ satisfaz $\rho(g) \circ T v=T \circ \pi(g) v$, assim temos o seguinte diagrama comutativo

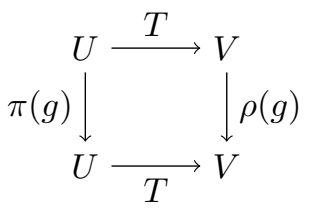

Veja que $T$ é um morfismo de G-módulos (homomorfismo entre grupos abelianos, com respeito à soma, que é $G$-equivariante). Dizemos que $U$ e $V$ são representações equivalentes se $T$ é um isomorfismo.

Lembre que um operador linear limitado $T$ em um espaço vetorial $V$ com produto interno é dito unitário se $T T^{*}=T^{*} T=I$, onde $I$ é o operador identidade em $V$ e $T^{*}$ é o operador adjunto de $T$, equivalentemente, $T$ é unitário se preserva o produto interno.

Definição 3.0.12. Se $V$ é um $G$-módulo, um produto interno euclidiano, $\langle\cdot, \cdot\rangle: V \times V \rightarrow \mathbb{R}$ é dito $G$-invariante se $\langle g u, g v\rangle=\langle u, v\rangle$ para todo $g \in G, u, v \in V$. Uma representação $V$ munida de um produto interno $G$-invariante é chamada representação unitária.

Observe que a representação $(V, \pi)$ tem um produto interno $G$-invariante, se e só se, $\pi(g) \in \operatorname{Aut}(V)$ é um operador unitário para todo $g \in G$. Se $G$ é um grupo compacto, então toda representação $V$ possui um produto interno $G$-invariante.

Definição 3.0.13. Dada uma representação $(V, \pi)$ de $G$, um subespaço $W \subset V$ que é G-invariante faz da restrição de $\pi(g) \grave{a} W$ um automorfismo de $W$ e dá origem ao homomorfismo

$$
\left.\pi\right|_{W}: G \longrightarrow G L(W),
$$

chamado sub-representação de $V$. Equivalentemente, se $V$ é um G-módulo e $W \subset V$ é $G$ invariante, $W$ é submódulo de $V$. Uma representação não nula $V$ é chamada irredutível se não tem outros submódulos além dos triviais $\{0\}$ e V. Uma representação que não é irredutível é dita redutivel.

Uma representação $\pi: G \longrightarrow G L(V)$ é dita soma direta (interna), se $V$ admite dois subespaços complementares $G$-invariantes. Dadas duas representações de $G,(V, \pi)$ e $(U, \rho)$ podemos, ainda, considerar a representação soma direta (externa) de $G$ em $V \oplus U$,

$$
\pi \oplus \rho: G \longrightarrow G L(V \oplus U)
$$

definida por

$$
(\pi \oplus \rho)(g)(v, u)=\pi(g) \oplus \rho(g)(v, u)=(\pi(g) v, \rho(g) u), \quad \forall v \in V, u \in U .
$$

Podemos considerar também o produto tensorial destas representações e obter a representação

$$
(\pi \otimes \rho): G \longrightarrow G L(V \otimes U)
$$


via

$$
(\pi \otimes \rho)(g)(v \otimes u)=(\pi(g) \otimes \rho(g))(v \otimes u)=\pi(g) v \otimes \rho(g) u, \quad \forall v \in V, u \in U .
$$

Proposição 3.0.14. Seja $G$ um grupo de Lie compacto. Se U é submódulo do G-módulo $V$, de dimensão finita, então existe submódulo complementar $W$ tal que $V=U \oplus W$. Todo G-módulo, de dimensão finita, é soma direta de submódulos irredutiveis.

Demonstração. Como $G$ é compacto, existe produto interno $\langle\cdot, \cdot\rangle, G$-invariante em $V$. Seja $W$ o complemento ortogonal de $U$ em $V$ com respeito a este produto interno, então $W$ é $G$-submódulo e $V=U \oplus W$. De fato, vamos mostrar que como $U$ é $G$-invariante, $U^{\perp}=W$ é $G$-invariante. Dado $w \in W$, para todo $g \in G$, temos

$$
\langle g w, u\rangle=\left\langle g^{-1} g w, g^{-1} u\right\rangle=\left\langle w, g^{-1} u\right\rangle=0,
$$

para todo $u \in U$. Logo $g w \in W$, para todo $w \in W$.

Para ver que $V$ pode ser escrito como soma direta de submódulos irredutíveis, proceda por indução sobre a dimensão de $V$. Se $V$ é irredutível, não há nada a fazer; se $V \neq\{0\}$ é redutível, então existe $U$ um $G$-submódulo próprio de $V$ que, como acabamos de ver, é complementado por um $G$-submódulo $W$ ortogonal a $U$ com respeito a um produto interno $G$-invariante escolhido e $V=U \oplus V$. Se $U$ e $W$ são irredutíveis, o resultado está provado, caso contrário, existem $G$ submódulos $U_{1}, U_{2}, W_{1}, W_{2}$, tais que $V=U_{1} \oplus U_{2} \oplus W_{1} \oplus W_{2}$, se as parcelas são irredutíveis, o resultado está provado, caso contrário, podemos proceder da mesma forma diversas vezes até obter $V=U_{1} \oplus \ldots \oplus U_{r} \oplus \ldots \oplus W_{1} \oplus \ldots \oplus W_{s}$, onde todos as parcelas são $G$-submódulos irredutíveis. Note que o processo é finito, uma vez que a dimensão de $V$ é finita.

Seja $G$ um grupo de Lie e $V$ uma representação irredutível de $G$ com produto interno $G$ invariante, segue do resultado acima que $V$ não pode ser escrito como soma direta de $G$-submódulos (ou, equivalentemente, de sub-representações).

\subsection{Ações Isométricas e Representações}

Seja $(M, g)$ uma variedade riemanniana compacta (com ou sem bordo). No espaço das funções $\mathcal{C}^{k}(M), \operatorname{com} k \geq 2$, definimos o operador laplaciano de $M$ com respeito à métrica riemanniana $g$ por

$$
\Delta_{g}=-\operatorname{div}_{g} \nabla_{g} .
$$

Os seguintes problemas de autovalor são clássicos em variedades compactas:

(1) Problema fechado: $\partial M=\emptyset$,

$$
\Delta f=\rho f, \text { em } M,
$$

(2) Problema de Dirichlet: $\partial M \neq \emptyset$,

$$
\left\{\begin{aligned}
\Delta f & =\rho f, \text { em } M \\
f & =0, \text { em } \partial M
\end{aligned}\right.
$$

(3) Problema de Neumann: $\partial M \neq \emptyset$,

$$
\left\{\begin{aligned}
\Delta f & =\rho f, \text { em } M, \\
\partial_{\eta} f & =0, \text { em } \partial M
\end{aligned}\right.
$$

onde $\eta$ é o campo unitário normal ao bordo.

Já vimos, na Subseção 1.5.7, sob o título O Operador Laplaciano, que $\Delta_{g}$ é simétrico com respeito ao produto $L^{2}(M)$ no espaço das funções $\mathcal{C}^{k}(M)$, com $k \geq 2$, que satisfazem qualquer das condições 
de bordo dadas nos problemas acima. No Teorema 1.5.3, vimos que para qualquer um desses problemas de autovalor, o laplaciano tem uma sequência infinita de autovalores não-negativos $\rho$, de multiplicidade geométrica finita

$$
0 \leq \rho_{0}<\rho_{1}<\ldots<\rho_{k}<\ldots,
$$

isto é, o autoespaço $V_{\rho}$ de $\Delta_{g}$ associado ao autovalor $\rho$, solução de um dos problemas acima, tem dimensão finita.

Considere, agora, um grupo de Lie $G$, agindo por isometrias, na variedade $(M, g)$ :

$$
\mu: G \times M \longrightarrow M
$$

O próximo resultado garante a invariância dos autoespaços do laplaciano de $M$ pela ação de $G$.

Proposição 3.1.1. Se $G$ é um grupo de Lie agindo por isometrias em $(M, g)$ e $V_{\rho}$ é um autoespaço do laplaciano $\Delta_{g}$ associado ao autovalor $\rho$, solução de algum dos problemas de autovalor acima, então $V_{\rho}$ é invariante pela ação de $G$.

Demonstração. Sejam $\xi \in G$ e $f \in V_{\rho} \subset \mathcal{C}^{k}(M), k \geq 2$, então

(a.) $f \circ \xi$ é uma função de classe $\mathcal{C}^{k}$ em $M$,

(b.) $\Delta_{g}(f \circ \xi)(x)=\left(\Delta_{g} f\right)(\xi(x))=\rho f(\xi(x))=\rho(f \circ \xi)(x), \forall x \in M$ (veja Apêndice C).

Além disso,

- se $\rho$ é solução do problema fechado e $f$ é uma autofunção associada a $\rho$, segue diretamente dos itens (a.) e (b.) acima que $f \circ \xi \in V_{\rho}$;

- se $\rho$ é solução do problema de Dirichlet, temos

$$
\left.(f \circ \xi)\right|_{\partial M}=\left.f(\xi)\right|_{\partial M}=0,
$$

ou seja, $f \circ \xi \in V_{\rho}$;

- se $\rho$ é solução do problema de Neumann, então

$$
\partial_{\eta}(f \circ \xi)=\left(\partial_{\eta} f\right) \circ \xi \cdot \partial_{\eta} \xi=0,
$$

donde $f \circ \xi \in V_{\rho}$,

o que conclui a prova.

Mais geralmente, se $F:(M, g) \longrightarrow(N, h)$ é uma isometria, então $(M, g)$ e $(N, h)$ têm o mesmo espectro e se $f$ é uma autofunção para $\Delta_{h}$, então $f \circ F$ é uma autofunção para $\Delta_{g}$ associada ao mesmo autovalor.

Veremos agora que a ação isométrica de um grupo de Lie $G$, numa variedade compacta $(M, g)$, determina antirrepresentações de $G$ nos autoespaços $V_{\rho}$ do laplaciano $\Delta_{g}$, via pull-back,

$$
f \longmapsto \xi^{*}(f)=f \circ \xi
$$

$\operatorname{com} \xi \in G$ e $f \in V_{\rho}$.

Proposição 3.1.2. Para cada autovalor $\rho$, solução de um dos problemas de autovalor acima citados,

$$
\pi_{\rho}: G \longrightarrow G L\left(V_{\rho}\right)
$$

definida pela aplicação linear

$$
\begin{aligned}
\pi_{\rho}(\xi): V_{\rho} & \longrightarrow V_{\rho} \\
f & \mapsto f \circ \xi
\end{aligned}
$$


é uma antirrepresentação de $G$ no autoespaço $V_{\rho}$.

Demonstração. Já vimos que os autoespaços do laplaciano são invariantes pela ação de $G$, resta mostrarmos que $\pi_{\rho}$ é um anti-homomorfismo de grupos de Lie. De fato, $\pi_{\rho}$ é suave e para todo $\xi, \zeta \in G, f \in V_{\rho}$, temos

$$
\begin{aligned}
\pi_{\rho}(\xi \zeta) f & =f \circ(\xi \zeta) \\
& =f \circ(\xi \circ \zeta) \\
& =(f \circ \xi) \circ \zeta \\
& =\pi_{\rho}(\zeta)(f \circ \xi) \\
& =\pi_{\rho}(\zeta)\left(\pi_{\rho}(\xi) f\right) \\
& =\pi_{\rho}(\zeta) \circ \pi_{\rho}(\xi) f
\end{aligned}
$$

e o resultado segue. 


\section{Parte II}

\section{Técnicas de Bifurcação Aplicadas ao Problema de Yamabe}





\section{Capítulo 1}

\section{Teoremas de Bifurcação Variacional}

\subsection{Motivação}

A Teoria de Bifurcação é o estudo de mudanças na estrutura qualitativa ou topológica de uma dada família, por exemplo, de soluções de uma família de equações diferenciais. Em geral, associase Teoria de Bifurcação a Sistemas Dinâmicos, uma linha de pesquisa da matemática que busca uma maneira determinística de descrever como um ponto, em um espaço geométrico apropriado, varia com o passar do tempo. Dada uma família de sistemas $X^{\prime}=F(\lambda, X)$, onde $\lambda \in \mathbb{R}$ é o parâmetro, ocorre uma bifurcação em $\lambda=\lambda_{0}$ quando há uma mudança significativa na estrutura das soluções em $\lambda-\varepsilon$ e $\lambda+\varepsilon$ para qualquer $\varepsilon>0$.

Exemplo 1.1.1. Seja $x=x(t)$ uma função a valores reais da variável $t \in \mathbb{R}$ e denote por $x^{\prime}(t)$ sua derivada. Considere a equação diferencial

$$
x^{\prime}(t)=\lambda x(t)
$$

onde $f_{\lambda}(x)=\lambda x(t)$ e $\lambda$ é um parâmetro real. Sabemos que $x(t)=k \mathrm{e}^{\lambda t}$ é solução da equação (1.1) para qualquer $k \in \mathbb{R}$, mais que isso, sabemos que essa solução é única (a menos da constante $k$ ). Um caso especial acontece para $k=0$ : a função constante igual a zero $x(t)=0$ é uma solução. Uma solução constante é chamada "solução (ou ponto) de equilíbrio" da equação (1.1). Note que para cada valor de $\lambda$ temos uma equação diferente e portanto, diferentes soluções. De fato, podemos descrever o comportamento das soluções se analisarmos o sinal de $\lambda$ :

- se $\lambda>0, \lim _{t \rightarrow \infty} k \mathrm{e}^{\lambda t}=+\infty$, se $k>0$ e $\lim _{t \rightarrow \infty} k \mathrm{e}^{\lambda t}=-\infty$, se $k<0$;

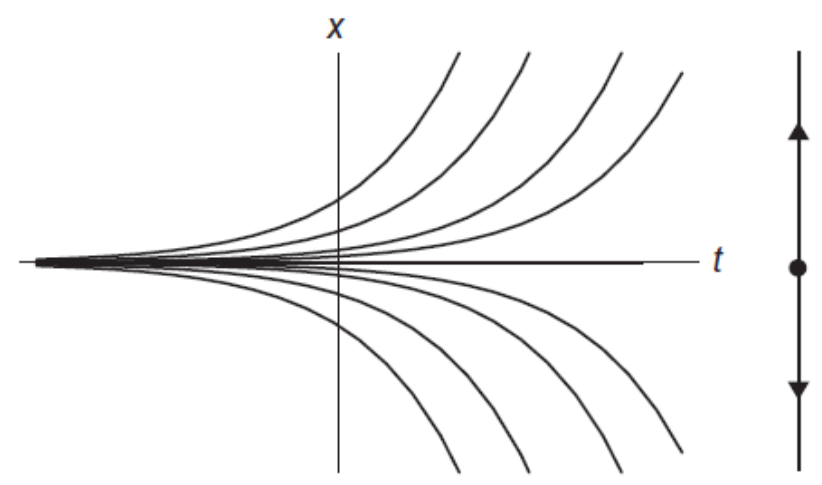

Figura 1.1: 
- se $\lambda=0$, então $x(t)=k$ é constante.

- se $\lambda<0, \lim _{t \rightarrow \infty} k \mathrm{e}^{\lambda t}=0$;

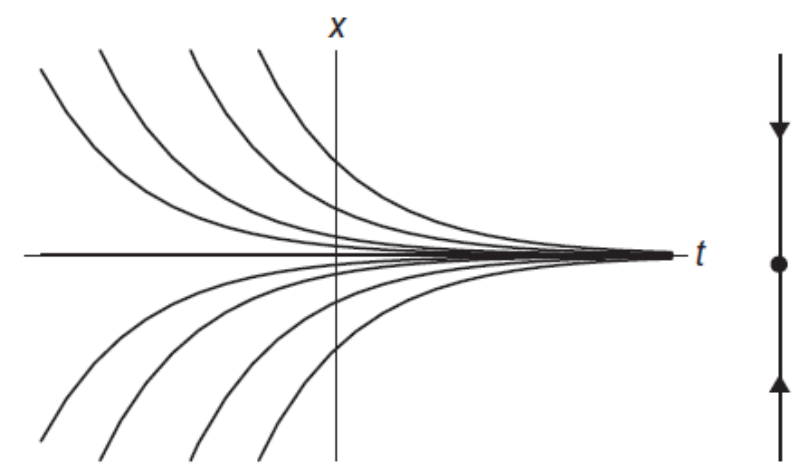

Figura 1.2:

Na verdade, o comportamento das soluções, em relação à solução de equilíbrio, varia bastante com o sinal de $\lambda$ a medida que o tempo passa. Agora, observe que se trocarmos $\lambda \neq 0$ por outro valor, $\beta$, com o mesmo sinal de $\lambda$, o comportamento qualitativo das soluções não muda. No entanto, se $\lambda=0$, qualquer alteração, por menor que seja, no parâmetro, muda radicalmente o comportamento das soluções. Dizemos que ocorre bifurcação em $\lambda=0$ para a família a 1-parâmetro $\lambda \longmapsto \lambda x(t)$.

O proposição abaixo dá condições de rigidez para a família contínua de funções $f_{\lambda+\varepsilon}$, isto é, condições sob as quais existe uma família de pontos $x_{0}(\varepsilon)$, variando continuamente, onde não há bifurcação para a família $\left\{f_{\lambda+\varepsilon}\right\}_{\varepsilon \in I}$.

Proposição 1.1.2. Considere a equação diferencial de $1^{a}$ ordem

$$
x^{\prime}=f_{\lambda}(x),
$$

e suponha que $f_{\lambda}\left(x_{0}\right)=0$ e $f_{\lambda}^{\prime}\left(x_{0}\right) \neq 0$. Então,

$$
x^{\prime}=f_{\lambda+\varepsilon}(x)
$$

tem ponto de equilıbrio em $x_{0}(\varepsilon)$, onde $\varepsilon \mapsto x_{0}(\varepsilon)$ é a função suave satisfazendo $x_{0}(0)=x_{0}$, para $\varepsilon$ suficientemente pequeno.

Demonstração. Defina $F(\varepsilon, x)=f_{\lambda+\varepsilon}(x)$, então $F\left(0, x_{0}\right)=f_{\lambda}\left(x_{0}\right)=0 \mathrm{e}$

$$
\frac{\partial F}{\partial x}\left(0, x_{0}\right)=f_{\lambda}^{\prime}\left(x_{0}\right) \neq 0 .
$$

Pelo Teorema da Função Implícita, existe um intervalo aberto $I$ contendo 0, uma vizinhança aberta $V$ contendo $x_{0}$ e uma aplicação

$$
\begin{array}{clcc}
x_{0}: & I \longrightarrow & V \\
\varepsilon & \longmapsto & x_{0}(\varepsilon)
\end{array}
$$

$\operatorname{com} x_{0}(0)=x_{0}$ tais que

$$
\left\{\left(\varepsilon, x_{0}(\varepsilon)\right): \varepsilon \in I\right\}=\{(\varepsilon, x) \in I \times V: F(\varepsilon, x)=0\},
$$


isto é, $F\left(\varepsilon, x_{0}(\varepsilon)\right)=0$. Mas, pela definição de $F$, temos

$$
F\left(\varepsilon, x_{0}(\varepsilon)\right)=f_{\lambda+\varepsilon}\left(x_{0}(\varepsilon)\right)
$$

$\operatorname{logo}, x_{0}(\varepsilon)$ é ponto de equilíbrio para a equação (1.2).

$O$ resultado acima diz que, se $f_{\lambda}^{\prime}\left(x_{0}\right) \neq 0$, então pequenas mudanças no parâmetro $\lambda$ não alteram a estrutura local em $x_{0}$. No caso de equações de primeira ordem, só ocorre bifurcação no caso não hiperbólico com $f_{\lambda}^{\prime}\left(x_{0}\right)=0$. A demonstração deste resultado ilustra de forma simples, o papel do Teorema da Função Implícita para se estabelecer rigidez local.

\subsection{Teoremas de Bifurcação Clássicos}

Em 1990, Smoller e Wasserman [40] usaram teoria de bifurcação para estudar as soluções da equação $M_{\lambda}(a)=0$, onde $M: \Lambda \times E \longrightarrow F$ é um operador suave, $E \subset F$ são espaços de Banach e $\Lambda \subset \mathbb{R}$ é um intervalo da reta e publicaram resultados, geral e equivariante, que passamos a exibir abaixo, sem muitos detalhes.

Sejam $B_{2}, B_{0}$ espaços de Banach e $H$ um espaço de Hilbert fixados tais que $B_{2} \subset B_{0} \subset H$ (cujas inclusões são contínuas). Seja $\Lambda$ um intervalo da reta e

$$
M: \Lambda \times B_{2} \longrightarrow B_{0}
$$

um operador suave de classe $\mathcal{C}^{1}$. Assuma que existe uma família contínua a 1-parâmetro $\lambda \mapsto$ $a_{\lambda} \in B_{2}$ satisfazendo $M_{\lambda}\left(a_{\lambda}\right)=0$, para todo $\lambda$ e seja $P_{\lambda}$ o subespaço vetorial fechado gerado pelo conjunto

$$
\left\{v \in B_{2}: \mathrm{d} M_{\lambda} v=\mu v, \text { para algum } \mu \geq 0, v \neq 0\right\},
$$

isto é, $P_{\lambda}$ é o espaço gerado pelos autovetores de $\mathrm{d} M_{\lambda}$ associados a autovalores não-negativos. Observe que $\mathrm{d} M_{\lambda} v=\mathrm{d} M_{\left(\lambda, a_{\lambda}\right)}(0, v), v \in T_{a_{\lambda}} B_{2}$.

Teorema 1.2.1 (Teorema de Bifurcação Abstrato). Seja $M$ o operador suave definido acima, tal que, para cada $\lambda \in \Lambda, M_{\lambda}$ é um operador gradiente (isto é, d $M_{\lambda}$ é simétrico) e satisfaz $M_{\lambda}(0)=0$, para todo $\lambda$. Assuma que existe $\varepsilon>0$, tal que

$$
\operatorname{dim} P_{\lambda, \varepsilon}=\left[\operatorname{dim}\left\{v \in B_{2}:\left(d M_{\lambda}+\varepsilon I\right) v=\mu v, \text { para algum } \mu \geq 0, v \neq 0\right\}\right]<\infty,
$$

para todo $\lambda \in \Lambda$. Se existir $\lambda_{1}<\lambda_{2}$ em $\Lambda$ satisfazendo

(i) $d M_{\lambda_{i}}$ é não singular, $i=1,2$, e

(ii) $P_{\lambda_{1}}$ não é isomorfo a $P_{\lambda_{2}}$.

Então existe $\lambda_{0} \in\left(\lambda_{1}, \lambda_{2}\right)$ tal que $\left(\lambda_{0}, 0\right)$ é um instante de bifurcação para a família $M_{\lambda}(a)=0$.

Como $P_{\lambda} \subset P_{\lambda, \varepsilon}$ a hipótese (1.3) implica que $\operatorname{dim} P_{\lambda_{i}}<\infty, i=1,2$, donde (ii) é equivalente a $\operatorname{dim} P_{\lambda_{1}} \neq \operatorname{dim} P_{\lambda_{2}}$.

Nem sempre é fácil verificar (ii) e pode ocorrer, ainda, de esta hipótese não ser satisfeita. Para esses casos, os autores apresentaram uma versão equivariante do Teorema, considerando a ação de um grupo de Lie no espaço funcional do problema, que deixa invariante o conjunto de soluções.

Seja $G$ um grupo de Lie compacto e fixe espaços de Banach $B_{2}, B_{0}$ e um espaço de Hilbert $H$, satisfazendo as inclusões contínuas $B_{2} \subset B_{0} \subset H$, todos invariantes sob a ação de $G$. Então, $G$ age também sobre a família de soluções $\left\{a_{\lambda}\right\}_{\lambda \in \Lambda}$. Suponha que $a_{\lambda}$ seja invariante sob a ação de $G$ para todo $\lambda \in \Lambda$. O objetivo principal de Smoller e Wasserman, no citado artigo, é estudar sob quais condições esta curva de soluções $G$-invariantes, também chamadas de soluções simétricas com respeito à ação de $G$, admite bifurcação para um ramo de soluções não invariantes, o que se chama de quebra de simetria. 
Seja $\Lambda$ um intervalo da reta e

$$
M: \Lambda \times B_{2} \longrightarrow B_{0},
$$

um operador suave de classe $\mathcal{C}^{1}, G$-invariante, isto é,

$$
M_{\lambda}(g \cdot a)=g \cdot M_{\lambda}(a), \quad \forall g \in G, a \in B_{2}, \lambda \in \Lambda,
$$

satisfazendo

$$
M_{\lambda}\left(a_{\lambda}\right)=0, \quad \forall \lambda \in \Lambda .
$$

Teorema 1.2.2 (Teorema de Bifurcação Equivariante). Considere o contexto e o operador $M$ como definidos acima. Suponha que existe $\lambda_{1}<\lambda_{2}$ em $\Lambda$ satisfazendo

(i) $d M_{\lambda_{i}}$ é não singular para $i=1,2$,

(ii) a representação de $O(n)$ em $P_{\lambda_{1}}$ é não equivalente à representação de $O(n)$ em $P_{\lambda_{2}}$.

Então existe um $\lambda_{0} \in\left(\lambda_{1}, \lambda_{2}\right)$ tal que $\left(\lambda_{0}, 0\right)$ é um instante de bifurcação para a família $M_{\lambda}(a)=0$.

O grupo $O(n)$ no item $(i i)$ do teorema, pode ser substituído por qualquer grupo nice no sentido que passamos a descrever abaixo.

Sejam $V_{1}$ e $V_{2}$ espaços de Hilbert de dimensão finita, $D_{1}\left(V_{i}\right)$ o disco unitário em $V_{i}, S_{1}\left(V_{i}\right)$ a esfera unitária em $V_{i}$, onde $i=1,2$. Seja $G$ um grupo de Lie que age por automorfismos em $V_{1}$ e $V_{2}$. Considere os subconjuntos

$$
B_{1}\left(V_{1}\right)=\frac{D_{1}\left(V_{1}\right)}{S_{1}\left(V_{1}\right)} \quad \text { e } \quad B_{1}\left(V_{2}\right)=\frac{D_{1}\left(V_{2}\right)}{S_{1}\left(V_{2}\right)}
$$

de $V_{1}$ e $V_{2}$ respectivamente. Note que estes conjuntos não passam do disco unitário com a fronteira colapsada em um único ponto, ou, dito de outra forma, a bola aberta união com um ponto do bordo.

Suponha que $B_{1}\left(V_{1}\right)$ e $B_{1}\left(V_{2}\right)$ têm o mesmo tipo homotópico $G$-equivariante, isto é, existem aplicações contínuas $G$-equivariantes $f: B_{1}\left(V_{1}\right) \longrightarrow B_{1}\left(V_{2}\right)$ e $h: B_{1}\left(V_{2}\right) \longrightarrow B_{1}\left(V_{1}\right)$ tais que $h \circ f \stackrel{H_{1}}{\simeq} i d_{B_{1}\left(V_{1}\right)}$ e $f \circ h \stackrel{H_{2}}{\simeq} i d_{B_{1}\left(V_{2}\right)}$; consequentemente, as homotopias $H_{1}$ e $H_{2}$ são $G$-equivariantes no sentido de $H_{i}\left(g \cdot v_{i}, t\right)=g \cdot H\left(v_{i}, t\right), i=1,2$. Então, temos a seguinte definição.

Definição 1.2.3. Um grupo $G$ é dito "nice" (de acordo com [40] ${ }^{1}$ ) se dadas quaisquer duas representações unitárias $\pi_{1}$ e $\pi_{2}$, em espaços $V_{1}$ e $V_{2}$ respectivamente, satisfazendo os termos acima, então essas representações são equivalentes.

\subsection{Teoremas de Bifurcação com Domínio Variável}

Em 2012, Lima, Piccione e Zedda [31] propuseram uma configuração mais geral para a qual os mesmos resultados ainda garantem bifurcação, definindo-os no espaço total de fibrados. Os autores consideraram uma família de operadores gradientes $F_{\lambda}$ definidos em subvariedades de Banach $\mathcal{D}_{\lambda}$ (de uma fixada variedade de Banach) que variam suavemente com $\lambda$, e tomam valores em subespaços fechados $\mathcal{E}_{\lambda}$ de um espaço de Banach, que variam suavemente com $\lambda$.

Apresentamos abaixo os Teoremas de Bifurcação para fibrados, nas versões geral e equivariante, cujas demonstrações consistem de aplicações diretas dos Teoremas 1.2.1 e 1.2.2 [40, Teoremas 2.1 e 3.1], respectivamente.

Definição 1.3.1. Dado um espaço de Banach B, uma família de subvariedades de Banach de B, $[a, b] \ni s \mapsto B_{s}$ é chamada de família $\mathcal{C}^{1}$ de subvariedades de $B$, se o conjunto

$$
\mathcal{B}=\left\{(x, s) \in B \times[a, b]: x \in B_{s}\right\}
$$

\footnotetext{
${ }^{1}$ Se $G_{0}$ é a componente conexa da identidade de um grupo de Lie $G, G$ é nice se $G / G_{0}=\{1\}$ ou se $G / G_{0}$ é o produto de um número finito de cópias de $\mathbb{Z}_{2}$ (ou de $\mathbb{Z}_{3}$ ).
} 
tem a estrutura de um subfibrado $\mathcal{C}^{1}$ do fibrado trivial $B \times[a, b]$. Analogamente, podemos definir uma familia $\mathcal{C}^{1}$ de subespaços fechados de $B$ como uma familia $[a, b] \ni s \mapsto S_{s}$ de subespaços de Banach de $B$ tal que o conjunto

$$
\mathcal{S}=\left\{(x, s) \in B \times[a, b]: x \in S_{s}\right\}
$$

é um subfibrado do fibrado trivial de Banach $B \times[a, b]$.

Note que, com as notações usadas na definição acima, se $s \mapsto x_{s} \in B$ é um caminho $\mathcal{C}^{1}$, $\mathcal{B}=\bigcup_{s \in[a, b]}\left(B_{s} \times\{s\}\right)$ é uma família $\mathcal{C}^{1}$ de subvariedades de $B$, com $x_{s} \in B_{s}$ para todo $s$, então o caminho $s \mapsto T_{x_{s}} S_{s}$ é uma família $\mathcal{C}^{1}$ de subespaços fechados de $B$.

Teorema 1.3.2 (Teorema de Bifurcação para Fibrados). Sejam $B_{0}$ e $B_{2}$ espaços de Banach e $H$ um espaço Hilbertizável. Considere as famílias $\mathcal{C}^{1}:\left\{\mathcal{D}_{s}\right\}_{s \in[a, b]}$ de subvariedades de Banach de $B_{2}$; $\left\{\mathcal{E}_{s}\right\}_{s \in[a, b]}$ de subespaços fechados $B_{0} ;$ e $\left\{\mathcal{H}_{s}\right\}_{s \in[a, b]}$ de subespaços fechados de $H$. Seja $F: \mathcal{D} \longrightarrow \mathcal{E}$ um morfismo entre fibrados e assuma que as seguintes hipóteses são satisfeitas:

a. $s \mapsto e_{s} \in \mathcal{E}_{s}$ é uma seção $\mathcal{C}^{1}$ do fibrado $\mathcal{E}$;

b. $s \mapsto d_{s} \in \mathcal{D}_{s}$ é uma seção $\mathcal{C}^{1}$ do fibrado $\mathcal{D}$ tal que, para todo $s \in[a, b]$,

$$
F\left(d_{s}, s\right)=\left(e_{s}, s\right)
$$

c. $s \mapsto\langle\cdot, \cdot\rangle_{s}$ é uma familia $\mathcal{C}^{1}$ de produtos internos completos em $\mathcal{H}_{s}$.

d. Existem inclusões contínuas $B_{2} \subset B_{0} \subset H$ que induzem inclusões $T_{d_{s}} \mathcal{D}_{s} \subset \mathcal{E}_{s} \subset \mathcal{H}_{s}$ para todo $s \in[a, b]$.

e. Além disso, para todo $s \in[a, b]$, assuma que a aplicação $F_{s}=F(\cdot, t): \mathcal{D}_{s} \longrightarrow \mathcal{E}_{s}$ é um operador gradiente em $d_{s}$, i.e. o diferencial $d F(\cdot, s): T_{d_{s}} \mathcal{D}_{s} \longrightarrow \mathcal{E}_{s}$ é simétrico com respeito ao produto interno $\langle\cdot, \cdot\rangle_{s}$.

f. O operador $d F(\cdot, s): T_{d_{s}} \mathcal{D}_{s} \longrightarrow \mathcal{E}_{s}$ é de Fredholm de indice zero para todo $s \in[a, b]$.

g. Suponha que exista uma base $\langle\cdot, \cdot\rangle_{s}$-ortonormal $e_{1}^{s}, e_{2}^{s}, \ldots$ de $\mathcal{H}_{s}$ consistindo de autovetores de $d F(\cdot, s)$;

h. os autovalores correspondentes têm multiplicidade finita e para todo $s \in[a, b]$, o número de autovalores negativos de $d F(\cdot, s)$, denotado por $n_{s}$, é finito.

i. Existe $s_{*} \in(a, b)$ tal que, para todo $\varepsilon>0$ suficientemente pequeno, temos

- $d F\left(\cdot, s_{*}-\varepsilon\right)$ e $d F\left(\cdot, s_{*}+\varepsilon\right)$ são não singulares,

- $n_{s_{*}-\varepsilon} \neq n_{s_{*}+\varepsilon}$.

Então $s_{*}$ é um instante de bifurcação para a equação $F(\cdot, s)=\left(e_{s}, s\right)$; isto é, existem sequências $d_{n} \in B_{2}$ e $s_{n} \in[a, b]$, com $d_{n} \in \mathcal{D}_{s_{n}}$ para todo $n$, tais que $\lim _{n \rightarrow \infty} s_{n}=s_{*}, \lim _{n \rightarrow \infty} d_{n}=d_{s_{*}}, d_{n} \neq d_{s_{n}}$ para todo $n$, e $F\left(d_{n}, s_{n}\right)=\left(e_{s_{n}}, s_{n}\right)$, para todo $n$.

Lembre que um morfismo de fibrados é uma aplicação contínua $F: \mathcal{D} \longrightarrow \mathcal{E}$ entre fibrados tal que $\pi_{\mathcal{E}} \circ F=\pi_{\mathcal{D}}$, onde $\pi$ é a respectiva projeção, e uma seção $\mathcal{C}^{1}$ de um fibrado $\mathcal{E}$ sobre um espaço base $J$ é uma aplicação de classe $\mathcal{C}^{1}, \xi: J \longrightarrow \mathcal{E}$ tal que $\pi_{\mathcal{E}}(\xi(s))=s$.

Teorema 1.3.3 (Teorema de Bifurcação Equivariante). Sejam $B_{0}$ e $B_{2}$ espaços de Banach e $H$ um espaço Hilbertizável. Considere as famílias $\mathcal{C}^{1}:\left\{\mathcal{D}_{s}\right\}_{s \in[a, b]}$ de subvariedades de Banach de $B_{2}$; $\left\{\mathcal{E}_{s}\right\}_{s \in[a, b]}$ de subespaços fechados $B_{0} ;$ e $\left\{\mathcal{H}_{s}\right\}_{s \in[a, b]}$ de subespaços fechados de $H$. Seja $F: \mathcal{D} \longrightarrow \mathcal{E}$ um morfismo entre fibrados e assuma que as seguintes hipóteses são satisfeitas: 
a. $s \mapsto e_{s} \in \mathcal{E}_{s}$ é uma seção $\mathcal{C}^{1}$ do fibrado $\mathcal{E} ;$

b. $s \mapsto d_{s} \in \mathcal{D}_{s}$ é uma seção $\mathcal{C}^{1}$ do fibrado $\mathcal{D}$ tal que, para todo $s \in[a, b]$,

$$
F\left(d_{s}, s\right)=\left(e_{s}, s\right)
$$

c. $s \mapsto\langle\cdot, \cdot\rangle_{s}$ é uma familia $\mathcal{C}^{1}$ de produtos internos completos em $\mathcal{H}_{s}$.

d. Existem inclusões contínuas $B_{2} \subset B_{0} \subset H$ que induzem inclusões $T_{d_{s}} \mathcal{D}_{s} \subset \mathcal{E}_{s} \subset \mathcal{H}_{s}$ para todo $s \in[a, b]$.

e. Além disso, para todo $s \in[a, b]$, assuma que a aplicação $F_{s}=F(\cdot, t): \mathcal{D}_{s} \longrightarrow \mathcal{E}_{s}$ é um operador gradiente em $d_{s}$, i.e. o diferencial $d F(\cdot, s): T_{d_{s}} \mathcal{D}_{s} \longrightarrow \mathcal{E}_{s}$ é simétrico com respeito ao produto interno $\langle\cdot, \cdot\rangle_{s}$.

f. O operador $d F(\cdot, s): T_{d_{s}} \mathcal{D}_{s} \longrightarrow \mathcal{E}_{s}$ é de Fredholm de indice zero para todo $s \in[a, b]$.

g. Suponha que exista uma base $\langle\cdot, \cdot\rangle_{s}$-ortonormal $e_{1}^{s}, e_{2}^{s}, \ldots$ de $\mathcal{H}_{s}$ consistindo de autovetores de $d F(\cdot, s)$;

h. os autovalores correspondentes têm multiplicidade finita e para todo $s \in[a, b]$, o número de autovalores negativos de $d F(\cdot, s)$, denotado por $n_{s}$, é finito.

i. Considere a ação de um grupo de Lie conexo (ou, mais geralmente, nice) $G$ agindo em $B_{0}$, $B_{2}$ e $H$, tal que $\mathcal{D}_{s}, \mathcal{E}_{s}, \mathcal{H}_{s}$ são $G$-invariantes e $F$ é $G$-equivariante.

j. Assuma que g.d $d_{s}=d_{s}$ e g.e $e_{s}=e_{s}$, para todo $g \in G$ e todo $s$.

$k$. É fácil ver que todo autoespaço de $d F(\cdot, s)$ é $G$-invariante para todo $s \in[a, b]$. Denote por $\tau_{s}^{-}$a representação de $G$ no espaço de dimensão finita dado pela soma direta de todos os autovalores de $d F(\cdot, s)$ que correspondem a autovalores negativos.

l. Seja $s_{*} \in(a, b)$ tal que, para todo $\varepsilon>0$ suficientemente pequeno, $d F\left(\cdot, s_{*}-\varepsilon\right), d F\left(\cdot, s_{*}+\varepsilon\right)$ são invertíveis e $\tau_{s_{*}-\varepsilon}^{-}, \tau_{s_{*}+\varepsilon}^{-}$são não equivalentes.

Então $s_{*}$ é um instante de bifurcação para a equação $F(\cdot, s)=\left(e_{s}, s\right)$; isto é, existem sequências $d_{n} \in B_{2}$ e $s_{n} \in[a, b]$, com $d_{n} \in \mathcal{D}_{s_{n}}$ para todo $n$, tais que $\lim _{n \rightarrow \infty} s_{n}=s_{*}, \lim _{n \rightarrow \infty} d_{n}=d_{s_{*}}, d_{n} \neq d_{s_{n}}$ para todo $n$, e $F\left(d_{n}, s_{n}\right)=\left(e_{s_{n}}, s_{n}\right)$, para todo $n$.

Lima, Piccione e Zedda, propuseram ainda, neste mesmo trabalho [31], um Teorema da Função Implícita neste mesmo contexto, resultado fundamental para obtenção de resultados de rigidez local (quando não há bifurcação).

Dados fibrados $\pi_{i}: E_{i} \rightarrow B_{i}, i=1,2$ e um morfismo de fibrados $M: E_{1} \rightarrow E_{2}$. A derivada vertical de $M$ em $e \in E_{1}$ é a aplicação linear

$$
\mathrm{d}_{\mathrm{ver}} M(e): T_{e} \mathcal{F}(e) \longrightarrow T_{M(e)} \mathcal{F}(M(e)),
$$

dada pelo diferencial da restrição $\left.M\right|_{\mathcal{F}(e)}: \mathcal{F}(e) \longrightarrow \mathcal{F}(M(e))$, onde $\mathcal{F}(e)=\pi_{1}^{-1}\left(\pi_{1}(e)\right) \subset E_{1}$ é a fibra de $E_{1}$ correspondente ao ponto $e$, e $\mathcal{F}(M(e))=\pi_{2}^{-1}\left(\pi_{2}(M(e))\right) \subset E_{2}$ é a fibra de $E_{2}$ correspondente a $M(e)$.

Teorema 1.3.4. Sejam $\pi_{i}: E_{i} \rightarrow B_{i}, i=1,2$, fibrados, e $M: E_{1} \rightarrow E_{2}$ um morfismo de fibrados de classe $\mathcal{C}^{k}$, com $k \geq 1$. Seja $s: U \subset B \rightarrow E_{2}$ uma seção local de $E_{2}$ de classe $\mathcal{C}^{k}$, com $U$ um subconjunto aberto de $B$ contendo $x_{0}, s\left(x_{0}\right)=e_{2}$, e seja $e_{1} \in M^{-1}\left(e_{2}\right)$. Assuma que a derivada vertical d ver $M\left(e_{1}\right)$ seja um isomorfismo. Então, existem vizinhanças abertas $V$ de $e_{1}$ em $E_{1}$, com $U^{\prime}=\pi_{1}(V) \in U$ e uma seção $\mathcal{C}^{k}, \tilde{s}: U^{\prime} \rightarrow E_{1}$ com $\tilde{s}\left(x_{0}\right)=e_{1}$, tal que e $\in V \cap M^{-1}(s(U))$ se e somente se $e \in \tilde{s}\left(U^{\prime}\right)$. 
Embora os autores se refiram a esta proposta como uma ligeira mudança de enunciado, fato é que o trabalho tem permitido importantes aplicações na Geometria, como demonstrado neste mesmo trabalho, no qual se estuda multiplicidade de soluções para o Problema de Yamabe em variedades produto compactas dentre outras coisas. 


\section{Capítulo 2}

\section{Bifurcação de Soluções para o Problema de Yamabe}

\subsection{O Problema de Yamabe}

O problema de Yamabe está ligado à ideia de se obter métricas canônicas em uma variedade diferenciável. Provavelmente, o resultado mais conhecido neste assunto seja o Teorema de Uniformização para superfícies simplesmente conexas que garante a existência de uma métrica de curvatura Gaussiana constante em cada classe conforme. Em dimensões mais altas, o matemático japonês Hidehiko Yamabe propôs uma conjectura que ficou conhecida como Problema de Yamabe:

Seja $\left(M, g_{0}\right)$ uma variedade riemanniana compacta de dimensão $m \geq 3$. Então existe uma métrica $g$ na classe conforme de $g_{0}$, segundo a qual $M$ tem curvatura escalar constante.

Dada uma métrica riemanniana $g_{0}$, qualquer métrica $g$ na classe conforme de $g_{0}$ pode ser escrita como $g=u^{\frac{4}{m-2}} g_{0}$, onde $u$ é uma função positiva suave. Se $R_{g_{0}}$ e $R_{g}$ denotam a curvatura escalar da variedade $M$ com respeito às métricas $g_{0}$ e $g$, respectivamente, então,

$$
\frac{m-2}{4(m-1)} R_{g} u^{\frac{m+2}{m-2}}=\Delta_{g_{0}} u+\frac{m-2}{4(m-1)} R_{g_{0}} u
$$

onde $\Delta_{g_{0}} u=-\operatorname{div}_{g_{0}}\left(\operatorname{grad}_{g_{0}} u\right)$ é o laplaciano geométrico, com grad $g_{0} u$ o gradiente de $u$ calculado com respeito à métrica $g_{0}$ e $\operatorname{div}_{g_{0}}$ é o divergente com respeito à métrica $g_{0}$. Assim, uma solução para o problema de Yamabe consiste de uma métrica $g=u^{\frac{4}{n-2}} g_{0}$, onde $u$ é uma solução positiva da equação diferencial parcial

$$
\Delta_{g_{0}} u+\frac{m-2}{4(m-1)} R_{g_{0}} u-K u^{\frac{m+2}{m-2}}=0
$$

com $K \in \mathbb{R}$, demonstrada na Seção 1.5 .

O problema de resolver a equação diferencial não linear (2.2) é equivalente ao problema variacional de estudar os pontos críticos do funcional de Hilbert-Einstein (H-E), normalizado,

$$
F(g)=\frac{\int_{M} R_{g} \omega_{g}}{\left(\int_{M} \omega_{g}\right)^{\frac{m-2}{m}}},
$$

restrito à classe conforme, denotada por $\left[g_{0}\right]$. O funcional $\mathrm{H}-\mathrm{E}$ é suave na variedade de Banach (de dimensão infinita) das métricas riemannianas de classe $\mathcal{C}^{k}$ em $M$, denotada por $\mathcal{M}^{k}(M)$. 
De fato, a expressão de $F$ restrito à classe conforme de $g_{0}$ é dada por

$$
F_{g_{0}}(u)=F\left(u^{\frac{4}{m-2}} g_{0}\right)=\frac{E(u)}{\|u\|_{p}^{2}},
$$

onde $p=\frac{2 m}{m-2}$,

$$
\text { e }
$$

$$
E(u)=\int_{M}\left(\left|\operatorname{grad}_{g_{0}} u\right|^{2}+\frac{m-2}{4(m-1)} R_{g_{0}} u^{2}\right) \omega_{g_{0}}
$$

$$
\|u\|_{p}=\left(\int_{M}|u|^{p} \omega_{g_{0}}\right)^{\frac{1}{p}}
$$

Calculando a primeira variação de $F_{g_{0}}$ na direção de $v \in \mathcal{C}^{k}(M)$, temos

$$
\delta F_{g_{0}}(u+t v)=\frac{2}{\|u\|_{p}^{2}} \int_{M}\left(\Delta_{g_{0}} u+\frac{m-2}{4(m-1)} R_{g_{0}} u-\frac{E(u)}{\|u\|_{p}^{p}} u^{\frac{m+2}{m-2}}\right) v \omega_{g_{0}}
$$

isto é, $u$ é ponto crítico de $F_{g_{0}}$ se e somente se satisfaz a $\operatorname{EDP}(2.2), \operatorname{com} K=\frac{E(u)}{\|u\|_{p}^{p}}$ e $p=\frac{2 m}{m-2}$.

Observe que estudar o funcional H-E normalizado restrito à classe conforme $\left[g_{0}\right]$ é equivalente a estudar o funcional H-E

$$
F(g)=\int_{M} R_{g} \omega_{g}
$$

restrito à classe conforme $\left[g_{0}\right]_{1}$, isto é, o conjunto das métricas conformes à métrica $g_{0}$ e de volume 1 .

Em 1960, foi publicado um artigo [41], atribuído à Yamabe, com uma prova para a conjectura. Contudo, o matemático australiano, Neil Trudinger, encontrou um erro na demonstração de Yamabe, o que não desmerece seu trabalho, afinal tratava-se de um problema tão difícil que levou mais de 16 anos para ser completamente resolvido. O próprio Trudinger só publicou resultados que complementavam o trabalho de Yamabe em 1968. Finalmente, em 1984, o esforço combinado de Aubin [2], Trudinger [39], Schoen [34], [35] e do próprio Yamabe, forneceu uma prova completa do resultado. Hoje sabemos que os pontos críticos do funcional $F$ restrito à variedade das métricas $\mathcal{C}^{k}$ de volume 1 sobre $M$, denotada por $\mathcal{M}_{1}^{k}(M)$, são as métricas de Einstein de volume 1; enquanto os pontos críticos de $F$ restrito à subvariedade $[g]_{1}$, das métricas $\mathcal{C}^{k}$, conformes a $g$, com volume 1 , são as métricas de curvatura escalar constante em $[g]_{1}$. O Problema de Yamabe deu origem a uma coleção de trabalhos concernentes à existência, unicidade e outros aspectos de suas soluções.

\subsection{Bifurcação de Soluções em Variedades Produto}

Técnicas de Bifurcação no problema de Yamabe têm sido usadas no caso de variedades riemannianas colapsadas, e no caso de métricas homogêneas em esferas, veja as referências [9] e [6]. No artigo [31], publicado em 2012, Lima, Piccione e Zedda estudaram rigidez local e multiplicidade de métricas de curvatura escalar constante em classes conformes de variedades produto compactas fazendo uso dos Teoremas 1.3.4 e 1.3.2, por eles propostos, decorrentes dos resultados de Smoller e Wasserman [40]. Nesta seção, descreveremos brevemente este trabalho que serviu de base para o nossos primeiros resultados, que serão apresentados na seção seguinte. Nesta seção utilizaremos a notação do citado artigo.

Seja $(M, g)$ uma variedade riemanniana compacta sem bordo de dimensão $m \geq 3$. Os autores provaram que, se $g_{0}$ é um ponto crítico do funcional H-E, restrito à classe conforme de métricas $\mathcal{C}^{k, \alpha}(M)^{1}, k \geq 3$, de volume $1, \mathcal{M}_{1}^{k, \alpha}(M, g)$, então $g_{0}$ é um ponto crítico não degenerado, se $\kappa_{g_{0}}=0$

\footnotetext{
${ }^{1}$ Aqui, $\alpha$ varia em $(0,1]$ e refere-se a uma condição de Hölder necessária para a validade dos resultados de rigidez
} 
ou se $\frac{\kappa_{g_{0}}}{m-1}$ não é um autovalor do operador Laplace-Beltrami $\Delta_{g_{0}}$, onde $\kappa_{g_{0}}$ denota a curvatura escalar com respeito à métrica $g_{0}$. Sob essas condições e usando o Teorema da Função Implícita para fibrados (1.3.4), os autores obtiveram um resultado de rigidez [31, Teorema 3.1], para uma família $\left\{g_{\lambda}\right\}_{\lambda \in[a, b]}$ de soluções do problema de Yamabe:

Se $g_{\lambda_{*}}$ é um ponto crítico não degenerado do funcional $H-E$, então a familia $\left\{g_{\lambda}\right\}_{\lambda}$ é localmente rígida no instante $\lambda_{*}$.

Isto significa que na classe conforme de $g_{\lambda_{*}}$ não existe outra métrica de curvatura escalar constante, diferente de $g_{\lambda_{*}}$, em uma vizinhança.

Um instante $\lambda \in(0,+\infty)$ para o qual a métrica $g_{\lambda}$ é degenerada é chamado de instante de degeneração. São esses os candidatos a instantes de bifurcação para a família $\left\{g_{\lambda}\right\}_{\lambda}$. Para entendermos melhor o significado de se obter um instante de bifurcação para uma família de soluções do problema de Yamabe, vejamos a seguinte definição.

Definição 2.2.1. Seja $M$ uma variedade riemanniana compacta (sem bordo) com dimensão $m \geq 3$ e seja $\left\{g_{\lambda}\right\}_{\lambda \in[a, b]}$ uma curva suave de métricas riemannianas de $M$ com curvatura escalar constante na variedade $\mathcal{M}^{k}(M)$. Um instante $\lambda_{*} \in[a, b]$ é chamado instante de bifurcação para a família $\left\{g_{\lambda}\right\}_{\lambda \in[a, b]}$ se existe uma sequência $\left(\lambda_{n}\right)_{n \geq 1}$ em $[a, b]$ e uma sequência de métricas riemannianas $\left(g_{n}\right)_{n \geq 1}$ em $\mathcal{M}^{k}(M)$ tais que

(a) para todo $n \geq 1, g_{n} \in\left[g_{\lambda_{n}}\right]$, mas $g_{n} \neq g_{\lambda_{n}}$;

(b) para todo $n \geq 1$, o volume de $M$ com respeito a $g_{n}$ coincide com o volume com respeito a $g_{\lambda_{n}}$

(c) para todo $n \geq 1, g_{n}$ tem curvatura escalar constante;

(d) $\lim _{n \rightarrow \infty} \lambda_{n}=\lambda_{*} e \lim _{n \rightarrow \infty} g_{n}=g_{\lambda_{*}}$ em $\mathcal{M}^{k}(M)$.

Se $\lambda_{*}$ não é um instante de bifurcação, então dizemos que a família é localmente rígida em $\lambda_{*}$.

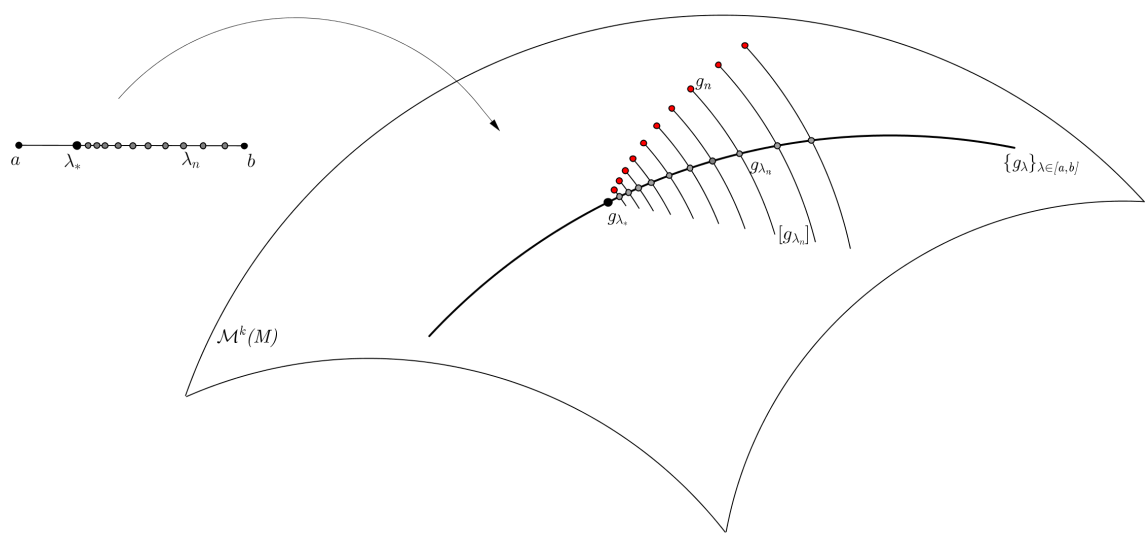

Figura 2.1:

Decorre da definição que encontrar um instante de bifurcação $\lambda_{*}$ para uma família de soluções do Problema de Yamabe, nos garante a existência de uma outro ramo $\left\{g_{n}\right\}_{n>0}$ de soluções completamente distintas do ramo trivial.

$\overline{\text { e bifurcação apresentados no citado trabalho. }}$ 
Seja $n_{\lambda}$ o número de autovalores do operador Laplace-Beltrami $\Delta_{\lambda}$ que é menor do que $\frac{\kappa_{\lambda}}{m-1}$. Segundo [31, Teorema 3.3], se $\left\{g_{\lambda}\right\}_{\lambda \in[a, b]}$ é rígida nos extremos do intervalo $[a, b]$ e $n_{a} \neq n_{b}$, existe instante de bifurcação $\lambda_{*} \in(a, b)$ para a família $\left\{g_{\lambda}\right\}_{\lambda}$.

Obtidos esses resultados, os autores estudaram a existência de instantes de bifurcação para a família de soluções construída da seguinte forma: Sejam $\left(M_{0}, g^{(0)}\right),\left(M_{1}, g^{(1)}\right)$ variedades riemannianas compactas (sem bordo) com curvaturas escalares constantes denotadas por $\kappa^{(0)}$ e $\kappa^{(1)}$ respectivamente. Sejam $m_{0}$ e $m_{1}$ suas respectivas dimensões, de modo que $m=m_{0}+m_{1} \geq 3$. Para cada $\lambda>0, g_{\lambda}=g^{(0)} \oplus \lambda g^{(1)}$ é uma métrica em $M=M_{0} \times M_{1}$. Então, $\left(M, g_{\lambda}\right)$ tem curvatura escalar constante $\kappa=\kappa^{(0)}+\frac{1}{\lambda} \kappa^{(1)}$, para todo $\lambda \in \Lambda$, isto é, $\left\{g_{\lambda}\right\}_{\lambda>0}$ é uma família de soluções para o problema de Yamabe na variedade produto $M$.

Nos instantes $\lambda$ tais que $g_{\lambda}$ é não degenerada, o resultado de rigidez do qual já falamos, garante unicidade local. Para curvaturas escalares positivas, ficou provado que, exceto por um número finito de instantes, chamados de instantes neutros, todos os demais instantes de degeneração são instantes de bifurcação. Mais que isso, os instantes de bifurcação consistem de duas sequências infinitas, uma tendendo a zero e a outra ilimitada.

Se as curvaturas escalares são ambas não positivas, então não existem instantes de bifurcação. Por outro lado, se $\kappa^{(0)} \leq 0$ e $\kappa^{(1)}>0$, todo instante de degeneração é um instante de bifurcação e consiste de uma sequência estritamente decrescente que converge para zero.

$\mathrm{O}$ artigo sugere uma maneira de abordar o caso dos instantes neutros usando o Teorema de Bifurcação Equivariante 1.3.3, considerando a ação (por isometrias) de um grupo de Lie conexo (ou nice, conforme discutido na seção 1.2), em um dos fatores da variedade produto $M$. Falaremos deste assunto com maiores detalhes na seção 3 .

\subsection{Bifurcação de Soluções em Variedades Produto com Bordo Mínimo}

Seja $(M, \bar{g})$ uma variedade riemanniana compacta com bordo $\partial M \neq \emptyset$ e dimensão $m \geq 3$, denote por $R_{\bar{g}}$ a curvatura escalar e $H_{\bar{g}}$ a curvatura média do bordo de $M$. O problema de Yamabe para variedades riemannianas compactas com bordo tem diferentes formulações que buscam a existência de uma métrica $\tilde{g}$ conforme a $\bar{g}$ tal que

(a.) $R_{\tilde{g}}$ é constante não nula e $H_{\tilde{g}}=0$;

(b.) $R_{\tilde{g}}=0$ e $H_{\tilde{g}}$ é constante não nula;

(c.) $R_{\tilde{g}}$ e $H_{\tilde{g}}$ são ambas constantes não nulas.

Os trabalhos de Cherrier [12], Escobar [18], [19], [20], Almaraz [1], Han [23] e outros certificam a existência de solução para os problemas acima para uma grande classe de variedades riemannianas com bordo.

O problema apresentado nesta seção está no contexto descrito pelo item (a.), acima:

Seja $\left(M_{1}, g^{(1)}\right)$ uma variedade riemanniana compacta, com $\partial M_{1}=\emptyset$, curvatura escalar constante e seja $\left(M_{2}, \bar{g}^{(2)}\right)$ uma variedade riemanniana compacta com bordo mínimo e curvatura escalar constante. Considere a variedade produto, $M=M_{1} \times M_{2}$, cujo bordo é dado por $\partial M=M_{1} \times \partial M_{2}$. Sejam $m_{1}$ e $m_{2}$ as dimensões de $M_{1}$ e $M_{2}$, respectivamente, e assuma que $\operatorname{dim}(M)=m=$ $m_{1}+m_{2} \geq 3$. Para cada $s \in(0,+\infty)$, defina $\bar{g}_{s}=g^{(1)} \oplus s \bar{g}^{(2)}$ uma família de métricas em $M$. Então, como vimos na Seção $1.5,\left(M, \bar{g}_{s}\right)$ tem curvatura escalar constante $R_{\bar{g}_{s}}$ e curvatura média $H_{\bar{g}_{s}}$ nula para todo $s>0$.

Em colaboração com o colega, Elkin Cárdenas Dario Diaz, professor da Universidad Del Cauca em Popayán, na Colômbia, estudamos bifurcação para a família de soluções $\left\{\bar{g}_{s}\right\}_{s}$ do problema 
de Yamabe em variedades produto com bordo mínimo, descrita acima. Este trabalhou introduziu alguns elementos novos no problema original, para variedades sem bordo [31], como a $\mathcal{C}^{k, \alpha}$-classe conforme normalizada,

$$
[\bar{g}]^{0}=\left\{\tilde{g} \in[\bar{g}]: H_{\tilde{g}}=0\right\}
$$

de uma dada métrica $\bar{g} \in \mathcal{M}^{k, \alpha}(M)$, que provamos ser uma subvariedade de $[\bar{g}]$, o resultado de rigidez local de [30, Proposição 3 e Corolário 4] que foi adaptado ao nosso problema e a condição de Neumann no bordo para o problema de autovalor do laplaciano de $\bar{g}$.

Nesta seção, apresentaremos a estrutura variacional do problema, dada essencialmente pelo funcional de Hilbert-Einstein restrito às classes conformes normalizadas (Subseção 2.3.1), os resultados sobre rigidez local e bifurcação que utilizamos (Subseção 2.3.2) e, finalmente, mostraremos que os principais resultados de Lima, Piccione e Zedda [31] se mantiveram válidos também no caso de variedades produto com bordo mínimo (Subseção 2.3.3).

\subsubsection{Variedades e Classes Conformes}

Seja $(M, \bar{g})$ uma variedade orientada compacta $m$-dimensional com bordo $\partial M \neq \emptyset, m \geq 3$. Como vimos no Exemplo 1.3.6 e na Seção 1.4, a métrica $\bar{g}$ induz produtos internos e normas em todos os espaços de tensores sobre $M$ e a conexão Levi-Civita $\bar{\nabla}$ associada a $\bar{g}$ induz uma conexão em todos os espaços vetoriais de campos tensoriais sobre $M$. Denotaremos por $\widehat{g}$ e $\widehat{\nabla}$ a métrica e a conexão induzidas, respectivamente. Assim, o espaço $\Gamma^{k}\left(T^{*} M \otimes T^{*} M\right)$ das seções $\mathcal{C}^{k}$ do fibrado vetorial $T^{*} M \otimes T^{*} M$ dos $(0,2)$ tensores simétricos de classe $\mathcal{C}^{k}$ em $M$ é um espaço de Banach com a norma

$$
\|\tau\|_{\mathcal{C}^{k}}=\max _{0 \leq|j| \leq k}\left[\max _{p \in M}\left\|\widehat{\nabla}^{j} \tau(p)\right\|_{\widehat{g}}\right],
$$

onde $j$ é um multi-índice (ver Seção 2.2), e portanto, é uma variedade de Banach.

Dado $k \geq 3$ e $\alpha \in(0,1]$, denote por $\mathcal{M}^{k, \alpha}(M)$ o conjunto de todas as métricas riemannianas de classe $\mathcal{C}^{k, \alpha}$ em $M$ (como definido na Seção 2.2), no sentido que os coeficientes da métrica são funções $\mathcal{C}^{k, \alpha}$ sobre $M$. O conjunto $\mathcal{M}^{k, \alpha}(M)$ é um cone aberto de $\Gamma^{k, \alpha}\left(T^{*} M \otimes T^{*} M\right)$, logo é, por si só, uma variedade de Banach e $T_{\bar{g}} \mathcal{M}^{k, \alpha}(M)=\Gamma^{k, \alpha}\left(T^{*} M \otimes T^{*} M\right)$, para uma métrica $\bar{g} \in \mathcal{M}^{k, \alpha}(M)$.

Considere o subconjunto aberto (vide Proposição 2.2.7) do espaço de Banach $\mathcal{C}^{k, \alpha}(M)$,

$$
\mathcal{C}_{+}^{k, \alpha}(M)=\left\{\phi \in \mathcal{C}^{k, \alpha}(M): \phi>0\right\}
$$

Agora, para cada $\bar{g} \in \mathcal{M}^{k, \alpha}(M)$, defina a $\mathcal{C}^{k, \alpha}$-classe conforme de $\bar{g}$ por

$$
[\bar{g}]:=\left\{\phi \bar{g}: \phi \in \mathcal{C}_{+}^{k, \alpha}(M)\right\}
$$

Proposição 2.3.1. A $\mathcal{C}^{k, \alpha}$-classe conforme de uma métrica riemanniana $\bar{g} \in \mathcal{M}^{k, \alpha}(M)$ é uma subvariedade mergulhada de $\mathcal{M}^{k, \alpha}(M)$.

Demonstração. Dada $\bar{g} \in \mathcal{M}^{k, \alpha}(M)$. Considere a aplicação injetiva

$$
\begin{aligned}
\mathcal{I}_{\bar{g}}: \mathcal{C}_{+}^{k, \alpha}(M) & \longrightarrow \mathcal{M}^{k, \alpha}(M) \\
\phi & \longmapsto \phi \bar{g}
\end{aligned}
$$

cuja imagem é a $\mathcal{C}^{k, \alpha}$-classe conforme de $\bar{g}$, $[\bar{g}]$. Observe que o diferencial

$$
\begin{aligned}
\left(\mathrm{d} \mathcal{I}_{\bar{g}}\right)_{\phi}: \mathcal{C}^{k, \alpha}(M) & \longrightarrow \Gamma^{k, \alpha}\left(T^{*} M \otimes T^{*} M\right) \\
\psi & \longmapsto \psi \bar{g}
\end{aligned}
$$


é injetor e tem inverso à esquerda dado pelo operador linear limitado

$$
\begin{aligned}
\mathcal{J}_{\bar{g}}: \Gamma^{k, \alpha}\left(T^{*} M \otimes T^{*} M\right) & \longrightarrow \mathcal{C}^{k, \alpha}(M) \\
h & \longmapsto \frac{1}{m} \operatorname{tr}_{\bar{g}} h
\end{aligned}
$$

de fato,

$$
\left\|\mathcal{J}_{\bar{g}} h\right\|=\left\|\frac{1}{m} \operatorname{tr}_{\bar{g}} h\right\|=\left|\frac{1}{m}\right|\left|\left\langle\bar{g}^{-1}, h\right\rangle\right| \leq m\left\|\bar{g}^{-1}\right\|\|h\|=M\|h\|
$$

e

$$
\mathcal{J}_{\bar{g}} \circ\left(\mathrm{d} \mathcal{I}_{\bar{g}}\right)_{\phi}(\psi)=\frac{1}{m} \operatorname{tr}_{\bar{g}}(\psi \bar{g})=\frac{1}{m} m \psi=\psi ;
$$

pela Proposição 2.0.10, a imagem do diferencial de $\mathcal{I}_{\bar{g}}$

$$
\operatorname{Im} d \mathcal{I}_{\bar{g}}=T_{\bar{g}}[\bar{g}]=\left\{\psi \bar{g}: \psi \in \mathcal{C}^{k, \alpha}(M)\right\},
$$

tem complemento fechado em $\Gamma^{k, \alpha}\left(T^{*} M \otimes T^{*} M\right)$, portanto $\operatorname{Im} \mathcal{I}_{\bar{g}}=[\bar{g}]$ é uma subvariedade mergulhada de $\mathcal{M}^{k, \alpha}(M)$.

Observação 2.3.2. Observe que a topologia induzida em $[\bar{g}]$, pela imersão $\mathcal{I}_{\bar{g}}$, definida acima, coincide com a topologia (do subespaço) induzida pela norma $\|\tau\|_{\mathcal{C}^{k}}$, do espaço dos (0,2)-tensores simétricos de classe $\mathcal{C}^{k}$ em $M$, uma vez que a norma usual no espaço das funções $\mathcal{C}^{k}$ definidas em $M$ é equivalente à norma $\|\tau\|_{\mathcal{C}^{k}}$. Com efeito, para cada $\phi \bar{g} \in[\bar{g}]$, temos

$$
\begin{aligned}
\|\phi \bar{g}\|_{\mathcal{C}^{k}} & =\max _{0 \leq|j| \leq k}\left[\max _{p \in M}\left\|\widehat{\nabla}^{j}(\phi \bar{g})(p)\right\|_{\widehat{g}}\right] \\
& =\max _{0 \leq|j| \leq k}\left[\max _{p \in M}\left\|\left(D^{j} \phi\right)_{p} \bar{g}_{p}+\phi(p) \widehat{\nabla}^{j} \bar{g}_{p}\right\|_{\widehat{g}}\right] \\
& =\max _{0 \leq|j| \leq k}\left[\max _{p \in M}\left|D^{j} \phi(p)\right|\left\|\bar{g}_{p}\right\|_{\widehat{g}}\right]
\end{aligned}
$$

portanto,

$$
\begin{aligned}
\min _{p \in M}\left\|\bar{g}_{p}\right\|_{\widehat{g}} \cdot \max _{0 \leq|j| \leq k}\left[\max _{p \in M}\left|D^{j} \phi(p)\right|\right] & \leq\|\phi \bar{g}\|_{\mathcal{C}^{k}} \leq \max _{p \in M}\left\|\bar{g}_{p}\right\|_{\widehat{g}} \cdot \max _{0 \leq|j| \leq k}\left[\max _{p \in M}\left|D^{j} \phi(p)\right|\right] \\
c_{1}\|\phi\|_{\mathcal{C}^{k}} & \leq\|\phi \bar{g}\|_{\mathcal{C}^{k}} \leq c_{2}\|\phi\|_{\mathcal{C}^{k}} .
\end{aligned}
$$

Isto justifica afirmarmos que a classe conforme $[\bar{g}]$, embora imagem de uma imersão, é subvariedade mergulhada de $\mathcal{M}^{k, \alpha}(M)$. Usaremos este fato outras vezes.

Em particular, $[\bar{g}]$ é uma variedade de Banach com estrutura diferencial induzida por $\mathcal{C}^{k, \alpha}(M)$, cujo espaço tangente

$$
T_{\bar{g}}[\bar{g}]=\left\{\psi \bar{g}: \psi \in \mathcal{C}^{k, \alpha}(M)\right\}
$$

que pode ser identificado $\operatorname{com} \mathcal{C}^{k, \alpha}(M)$.

Para cada $\bar{g} \in \mathcal{M}^{k, \alpha}(M)$, denote por $R i c_{\bar{g}}$ a curvatura de Ricci e $R_{\bar{g}}$ a curvatura escalar com respeito a $\bar{g}$. Seja $\eta_{\bar{g}}$ o campo unitário (que aponta para dentro) normal a $\partial M$; denote por $H_{\bar{g}}$ a curvatura média do bordo induzida por $\bar{g}$. Seja $\omega_{\bar{g}}$ a forma volume em $M$, com respeito a $\bar{g}$, e $\sigma_{\bar{g}}$ a forma volume induzida em $\partial M$.

A função volume definida em $\mathcal{M}^{k, \alpha}(M)$ é dada por

$$
\mathcal{V}(\bar{g}):=\int_{M} \omega_{\bar{g}}
$$

Observe (Apêndice A) que $\mathcal{V}$ é suave e para cada $\bar{g} \in \mathcal{M}^{k, \alpha}(M)$ e $h \in T_{\bar{g}} \mathcal{M}^{k, \alpha}(M)$ seu diferencial 
é dado por

$$
(d \mathcal{V})_{\bar{g}}(h)=\frac{1}{2} \int_{M} \operatorname{tr}_{\bar{g}}(h) \omega_{\bar{g}}
$$

Definimos

$$
\mathcal{M}^{k, \alpha}(M)_{1}:=\left\{\bar{g} \in \mathcal{M}^{k, \alpha}(M): \mathcal{V}(\bar{g})=1\right\}
$$

o subconjunto de $\mathcal{M}^{k, \alpha}(M)$ que consiste das métricas de volume 1.

Proposição 2.3.3. $\mathcal{M}^{k, \alpha}(M)_{1}$ é uma subvariedade mergulhada de $\mathcal{M}^{k, \alpha}(M)$.

Demonstração. Considere a função volume $\mathcal{V}: \mathcal{M}^{k, \alpha}(M) \longrightarrow \mathbb{R}$. É claro que $\mathcal{V}^{(-1)}(\{1\})=$ $\mathcal{M}^{k, \alpha}(M)_{1}$. Seja $\bar{g} \in \mathcal{M}^{k, \alpha}(M)_{1}$, tomando $h=\bar{g}$ em (2.5), então o diferencial

$$
(d \mathcal{V})_{\bar{g}}(\bar{g})=\frac{1}{2} \int_{M} \operatorname{tr}_{\bar{g}}(\bar{g}) \omega_{\bar{g}}=\frac{m}{2} \mathcal{V}(\bar{g})=\frac{m}{2} \neq 0,
$$

é sobrejetivo. Portanto, $\mathcal{M}^{k, \alpha}(M)_{1}$ é a imagem inversa de um valor regular. Além disso, o núcleo (que coincide com o espaço tangente $T_{\bar{g}} \mathcal{M}^{k, \alpha}(M)_{1}$ ),

$$
\operatorname{ker}\left\{(d \mathcal{V})_{\bar{g}}\right\}=\left\{h \in T_{\bar{g}} \mathcal{M}^{k, \alpha}(M): \int_{M} \operatorname{tr}_{\bar{g}}(h) \omega_{\bar{g}}=0\right\}
$$

tem complemento fechado em $\Gamma^{k, \alpha}\left(T^{*} M \oplus T^{*} M\right)$. Com efeito, dado $h$, um $(0,2)$-tensor simétrico de classe $\mathcal{C}^{k, \alpha}$, defina

$$
f=h-\left(\frac{1}{m} \int_{M} \operatorname{tr}_{\bar{g}} h \omega_{\bar{g}}\right) \bar{g}
$$

então $f \in \operatorname{ker}\left\{(d \mathcal{V})_{\bar{g}}\right\}$,

$$
\begin{aligned}
(d \mathcal{V})_{\bar{g}}(f) & =\frac{1}{2} \int_{M} \operatorname{tr}_{\bar{g}}(h-\underbrace{\left(\frac{1}{m} \int_{M} \operatorname{tr}_{\bar{g}} h \omega_{\bar{g}}\right)}_{\in \mathbb{R}} \bar{g}) \omega_{\bar{g}} \\
& =\frac{1}{2} \int_{M} \operatorname{tr}_{\bar{g}} h \omega_{\bar{g}}-\frac{1}{2}\left(\frac{1}{m} \int_{M} \operatorname{tr}_{\bar{g}} h \omega_{\bar{g}}\right) \int_{M} \operatorname{tr}_{\bar{g}} \bar{g} \omega_{\bar{g}} \\
& =\frac{1}{2} \int_{M} \operatorname{tr}_{\bar{g}} h \omega_{\bar{g}}-\frac{1}{2}\left(\frac{1}{\not \varkappa} \int_{M} \operatorname{tr} h \omega_{\bar{g}}\right) \not \varkappa \int_{M} \omega_{\bar{g}} \\
& =0
\end{aligned}
$$

e $h=f+\left(\frac{1}{m} \int_{M} \operatorname{tr}_{\bar{g}} h \omega_{\bar{g}}\right) \bar{g}$, isto é, $h$ é escrito como soma de um elemento do núcleo e um elemento do subespaço formado pelos múltiplos escalares do tensor métrico $\alpha \bar{g}, \alpha \in \mathbb{R}$, que é fechado. O resultado segue.

Agora, se $\bar{g} \in \mathcal{M}^{k, \alpha}(M)_{1}$, a métrica $\phi \bar{g} \in[\bar{g}]$ não pertence, necessariamente, a $\mathcal{M}^{k, \alpha}(M)_{1}$. De fato, como mostramos na Seção 1.5,

$$
\mathcal{V}(\phi \bar{g})=\int_{M} \omega_{\phi \bar{g}}=\int_{M} \phi^{\frac{m}{2}} \omega_{\bar{g}} .
$$

Observe que, se $\bar{g}$ tem curvatura escalar constante e volume $1, R_{s \bar{g}}=\frac{1}{s} R_{\bar{g}}$, com $s \geq 2$, tem curvatura escalar constante, mas $\mathcal{V}(s \bar{g}) \neq 1$. Gostaríamos de eliminar esses casos para tratar questões relacionadas à unicidade. Assim, para cada $\bar{g} \in \mathcal{M}^{k, \alpha}(M)_{1}$, definimos a classe conforme 
de volume 1 ,

$$
[\bar{g}]_{1}=\left\{\phi \bar{g}: \phi \in \mathcal{C}_{+}^{k, \alpha}(M), \int_{M} \phi^{\frac{m}{2}} \omega_{\bar{g}}=1\right\}
$$

que é uma subvariedade mergulhada de $[\bar{g}]$ (a prova é análoga à prova da Proposição anterior). Além disso, $[\bar{g}]_{1}$ é uma subvariedade mergulhada de $\mathcal{M}^{k, \alpha}(M)_{1}$, como provamos abaixo (em [30] há outra prova deste fato).

Proposição 2.3.4. A $\mathcal{C}^{k, \alpha}$-classe conforme de volume $1,[\bar{g}]_{1}$, é uma subvariedade mergulhada de $\mathcal{M}^{k, \alpha}(M)_{1}$. Mais ainda,

$$
T_{\bar{g}}[\bar{g}]_{1}=\left\{\psi \bar{g}: \psi \in \mathcal{C}^{k, \alpha}(M), \int_{M} \psi \omega_{\bar{g}}=0\right\} .
$$

Demonstração. Como sabemos que $\mathcal{C}_{+}^{k, \alpha}(M)$ é uma variedade de Banach, para cada $\bar{g} \in \mathcal{M}^{k, \alpha}(M)_{1}$, podemos definir $\mathcal{V}_{\bar{g}}: \mathcal{C}_{+}^{k, \alpha}(M) \longrightarrow \mathbb{R}$ como a função suave

$$
\mathcal{V}_{\bar{g}}(\phi):=\mathcal{V}(\phi \bar{g}),
$$

cujo diferencial, para cada $\psi \in T_{\phi} \mathcal{C}_{+}^{k, \alpha}(M)=\mathcal{C}^{k, \alpha}(M)$ é dado por

$$
\begin{aligned}
\left(d \mathcal{V}_{\bar{g}}\right)_{\phi}(\psi) & =(d \mathcal{V})_{\phi \bar{g}}(\psi) \\
& =\left.\frac{d}{d t}\right|_{t=0} \int_{M}(\phi+t \psi)^{\frac{m}{2}} \omega_{\bar{g}} \\
& =\left.\frac{m}{2} \int_{M}(\phi+t \psi)^{\frac{m}{2}-1} \psi \omega_{\bar{g}}\right|_{t=0} \\
& =\frac{m}{2} \int_{M} \phi^{\frac{m}{2}-1} \psi \omega_{\bar{g}} .
\end{aligned}
$$

Seja $\phi \in \mathcal{C}_{+}^{k, \alpha}(M)$ tal que $\mathcal{V}_{\bar{g}}(\phi)=1$. Se $\psi=\phi$, segue que $\left(d \mathcal{V}_{\bar{g}}\right)_{\phi}(\phi)=\frac{m}{2} \neq 0$, i.e., $\left(d \mathcal{V}_{\bar{g}}\right)_{\phi}$ é sobrejetivo; além disso,

$$
\begin{aligned}
\operatorname{ker}\left(d \mathcal{V}_{\bar{g}}\right)_{\phi} & =\left\{\psi \in \mathcal{C}^{k, \alpha}(M): \frac{m}{2} \int_{M} \phi^{\frac{m}{2}-1} \psi \omega_{\bar{g}}=0\right\} \\
& =\left\{\psi \in \mathcal{C}^{k, \alpha}(M): \frac{m}{2} \int_{M}\left(\phi^{-1} \psi\right) \phi^{\frac{m}{2}} \omega_{\bar{g}}=0\right\} \\
& =\left\{\psi \in \mathcal{C}^{k, \alpha}(M): \frac{m}{2} \int_{M} \phi^{-1} \psi \omega_{\phi \bar{g}}=0\right\}
\end{aligned}
$$

isto é, $\operatorname{ker}\left(d \mathcal{V}_{\bar{g}}\right)_{\phi}$ é o complemento ortogonal, com respeito ao produto interno induzido pela métrica $\phi \bar{g}$ no espaço $L^{2}(M)$, do subespaço fechado

$$
\left\{\varphi \in \mathcal{C}^{k, \alpha}(M): \varphi=\lambda \phi^{-1}, \lambda \in \mathbb{R}\right\},
$$

no ponto $\phi \in \mathcal{C}^{k, \alpha}(M)$. Portanto, $\mathcal{V}_{\bar{g}}^{-1}(1)$ é uma subvariedade mergulhada de $\mathcal{C}_{+}^{k, \alpha}(M)$.

Agora, para $\bar{g} \in \mathcal{M}^{k, \alpha}(M)_{1}$, defina as aplicações suaves $S: \mathcal{V}_{\bar{g}}^{-1}(\{1\}) \longrightarrow \mathcal{M}^{k, \alpha}(M)_{1} \mathrm{e}$ $T: \mathcal{M}^{k, \alpha}(M)_{1} \longrightarrow \mathcal{C}^{k, \alpha}(M)$ por

$$
S(\phi)=\phi \bar{g} \quad \text { and } \quad T(\tilde{g})=\frac{1}{m} \operatorname{tr}_{\bar{g}}(\tilde{g}) .
$$

Observe que $S$ é uma imersão injetiva e $T$ é inversa à esquerda de $S$ (análogo à (2.4)), logo $\operatorname{Im}(d S)_{\phi}$ tem complemento fechado. Portanto, $[\bar{g}]_{1}=\operatorname{Im} S$ é uma subvariedade mergulhada de $\mathcal{M}^{k, \alpha}(M)_{1}$. 
Finalmente, se tomarmos $\phi=1$ (que é quando teremos $S(1)=\bar{g}$ ), temos

$$
T_{1} \mathcal{V}_{\bar{g}}^{-1}(1)=\left\{\psi \in \mathcal{C}^{k, \alpha}(M): \int_{M} \psi \omega_{\bar{g}}=0\right\}
$$

Como $S$ é uma imersão, $T_{\bar{g}}[\bar{g}]_{1}=\operatorname{Im}(d S)_{1}$, mas $(d S)_{\phi}(\psi)=\psi \bar{g}$, para todo $\phi \in \mathcal{V}_{\bar{g}}^{-1}(1), \psi \in$ $T_{\phi} \mathcal{V}_{\bar{g}}^{-1}(1)$; então obtemos a expressão (2.6) para o espaço tangente, que pode ser identificado com

$$
T_{\bar{g}}[\bar{g}]_{1}=\left\{\psi \in \mathcal{C}^{k, \alpha}(M): \int_{M} \psi \omega_{\bar{g}}=0\right\} .
$$

Note que $[\bar{g}]_{1}=\mathcal{M}^{k, \alpha}(M)_{1} \cap[\bar{g}]$. Segundo [30], $\mathcal{M}^{k, \alpha}(M)_{1}$ é transversal a $[\bar{g}]$, então $[\bar{g}]_{1}$ é uma subvariedade mergulhada de $\mathcal{M}^{k, \alpha}(M)$.

Agora, defina a $\mathcal{C}^{k, \alpha}$-classe conforme normalizada da métrica $\bar{g} \in \mathcal{M}^{k, \alpha}(M)$ por

$$
[\bar{g}]^{0}=\left\{\tilde{g} \in[\bar{g}]: H_{\tilde{g}}=0\right\} .
$$

Este conjunto é não vazio. Com efeito, um resultado obtido por Escobar em [18] assegura que existe pelo menos uma métrica com curvatura média nula em cada classe conforme, portanto, dada uma classe conforme $[\bar{g}]$ seja $\tilde{g} \in[\bar{g}]$ uma métrica de curvatura média nula. Ora, $[\bar{g}] \equiv[\tilde{g}]$, logo podemos assumir que $H_{\bar{g}}=0$.

Proposição 2.3.5. A $\mathcal{C}^{k, \alpha}$-classe conforme normalizada de $\bar{g}$ pode ser identificada com

$$
\mathcal{C}_{+}^{k, \alpha}(M)^{0}=\left\{\phi \in \mathcal{C}_{+}^{k, \alpha}(M): \partial_{\eta_{\bar{g}}} \phi=0 \text { em } \partial M\right\}
$$

que é um subconjunto fechado de $\mathcal{C}^{k, \alpha}(M)$.

Demonstração. Seja $\tilde{g}=\phi^{\frac{4}{m-2}} \bar{g} \in[\bar{g}]^{0}$. Já vimos, na Seção 1.5.5, que

$$
H_{\tilde{g}}=\phi^{-\frac{m}{m-2}}\left(H_{\bar{g}} \phi+\frac{2(m-1)}{(m-2)} \partial_{\eta_{\bar{g}}} \phi\right)
$$

como $H_{\bar{g}}=0$ e $\phi>0$, segue que $H_{\tilde{g}}=0$ se e só se $\partial_{\eta_{\bar{g}}} \phi=0$. Agora, se $\left(\phi_{n}\right)_{n}$ é uma sequência de funções em $\mathcal{C}_{+}^{k, \alpha}(M)^{0}$ convergindo para uma função $\phi$ na norma $\mathcal{C}^{k, \alpha}$, então para todo $n$ maior que um certo $n_{0}$, temos

$$
\begin{aligned}
\left\|\phi_{n}-\phi\right\|_{\mathcal{C}^{k, \alpha}}<\varepsilon & \Rightarrow\left\|\phi_{n}-\phi\right\|_{\mathcal{C}^{k}}<\varepsilon \\
& \Rightarrow \max _{0 \leq|a| \leq k}\left[\max _{p \in M}\left|D^{a}\left(\phi_{n}-\phi\right)(p)\right|\right]<\varepsilon \\
& \Rightarrow \max _{p \in M}\left|\partial_{\eta_{\bar{g}}} \phi_{n}(p)-\partial_{\eta_{\bar{g}}} \phi(p)\right|<\varepsilon \\
& \Rightarrow\left\|\partial_{\eta_{\bar{g}}} \phi_{n}-\partial_{\eta_{\bar{g}}} \phi\right\|_{\mathcal{C}^{0}}<\varepsilon
\end{aligned}
$$

como $\partial_{\eta_{\bar{g}}} \phi_{n}=0$ para todo $n$, segue que $\partial_{\eta_{\bar{g}}} \phi=0$, portanto $\phi \in \mathcal{C}_{+}^{k, \alpha}(M)^{0}$, o que conclui a prova.

Queremos mostrar que a $\mathcal{C}^{k, \alpha}$-classe conforme normalizada, $[\bar{g}]^{0}$, é uma subvariedade da $\mathcal{C}^{k, \alpha_{-}}$ classe conforme, $[\bar{g}]$. Para isto, precisaremos do fato provado na proposição abaixo, que é uma versão elementar (ver Apêndice B) de um resultado mais geral que pode ser encontrado em [37, Teorema 4, Cap. IV]. 
Proposição 2.3.6. Existe uma aplicação linear contínua

$$
\mathcal{F}: \mathcal{C}^{k, \alpha}(\partial M) \longrightarrow \mathcal{C}^{k+1, \alpha}(M)
$$

tal que, para $\xi \in \mathcal{C}^{k, \alpha}(\partial M)$ as seguintes propriedades são satisfeitas:

(i.) $\mathcal{F}(\xi)$ se anula em $\partial M$;

(ii.) $\partial_{\eta} \mathcal{F}(\xi)=\xi$.

Demonstração. Escolha um conjunto finito de cartas locais $\left(U_{r}, \varphi_{r}\right), r=1, \ldots, n$ em $M$ satisfazendo as seguintes propriedades:

(a.) $U_{r}$ é um subconjunto aberto de $M$, com $U_{r} \cap \partial M \neq \emptyset$ para todo $r$;

(b.) $U:=\bigcup_{r=1}^{n} U_{r}$ é uma vizinhança aberta de $\partial M$;

(c.) $\varphi_{r}$ é um difeomorfismo de $U_{r}$ para $\mathbb{R}^{m-1} \times[0,+\infty)$ que leva $U_{r} \cap \partial M$ sobre $\mathbb{R}^{m-1} \times\{0\}$;

(d.) $\left(d \varphi_{r}\right)_{p}\left(\eta_{p}\right)=\frac{\partial}{\partial x_{m}}$, para todo $p \in U_{r} \cap \partial M$.

Seja $U_{0}=M \backslash \partial M$, então $\left\{U_{r}\right\}_{r=0}^{n}$ é uma cobertura aberta de $M$, e tome uma partição da unidade, suave, $\left\{\rho_{r}\right\}_{r=0}^{n}$ subordinada a esta cobertura. Dada $\xi \in \mathcal{C}^{k, \alpha}(\partial M)$, para todo $r$, considere a função $\xi_{r}=\left.\xi \circ \varphi_{r}^{-1}\right|_{\mathbb{R}^{m-1} \times\{0\}}: \mathbb{R}^{m-1} \times\{0\} \longrightarrow \mathbb{R}$, que é de classe $\mathcal{C}^{k, \alpha}$. Identificando $\mathbb{R}^{m-1} \times\{0\}$ com $\mathbb{R}^{m-1}$, defina $F_{\xi_{r}}: \mathbb{R}^{m} \longrightarrow \mathbb{R}$, por partes, como

$$
F_{\xi_{r}}(x)= \begin{cases}\frac{1}{\left(x_{m}\right)^{m-1}} \int_{Q(x)} \xi_{r}(z) d z, & \text { se } x_{m} \neq 0 \\ 0, & \text { se } x_{m}=0\end{cases}
$$

onde, $x=\left(x_{1}, \ldots, x_{m-1}, x_{m}\right), z=\left(z_{1}, \ldots, z_{m-1}\right)$ e $Q(x)=\prod_{i=1}^{m-1}\left[x_{i}-\frac{1}{2} x_{m}, x_{i}+\frac{1}{2} x_{m}\right]$. A aplicação $F_{\xi_{r}}$ é de classe $\mathcal{C}^{k+1, \alpha}$ em $\mathbb{R}^{m}$. Agora,

$$
\varphi_{r}: U_{r} \longrightarrow \mathbb{R}^{m-1} \times[0,+\infty) \subset \mathbb{R}^{m},
$$

daí, podemos considerar a seguinte composição de funções: $\mathcal{F}_{r}=F_{\xi_{r}} \circ \varphi_{r}$ de classe $\mathcal{C}^{k+1, \alpha}$ definida no aberto $U_{r}$, para todo $r=1, \ldots, n$. Usando partições da unidade, colamos todas essas funções definidas localmente, para finalmente obtermos $\mathcal{F}(\xi): M \longrightarrow \mathbb{R}$ definida por

$$
\mathcal{F}(\xi):=\left\{\begin{array}{ll}
\sum_{r=1}^{n} \rho_{r} \cdot \mathcal{F}_{r}, & \text { se } p \in U \\
0, & \text { se } p \in M \backslash U
\end{array} .\right.
$$

Vamos verificar que $\mathcal{F}(\xi)$ satisfaz as propriedades desejadas. Por construção, $\mathcal{F}(\xi) \in \mathcal{C}^{k+1, \alpha}(M)$, além disso

(i.) se $p \in \partial M$, temos

$$
\begin{aligned}
\mathcal{F}(\xi)(p) & =\sum_{r=1}^{n}\left(\rho_{r} \cdot \mathcal{F}_{r}\right)(p)=\sum_{r=1}^{n} \rho_{r}(p) \cdot \mathcal{F}_{r}(p) \\
& =\sum_{r=1}^{n} \rho_{r}(p) \cdot\left(F_{\xi_{r}} \circ \varphi_{r}\right)(p)=\sum_{r=1}^{n} \rho_{r}(p) \cdot F_{\xi_{r}}\left(\varphi_{r}(p)\right) \\
& =\sum_{r=1}^{n} \rho_{r}(p) \cdot F_{\xi_{r}}\left(x_{1}, \ldots, x_{m-1}, 0\right)=0
\end{aligned}
$$


(ii.) para todo $p \in \partial M$, vale

$$
\begin{aligned}
\partial_{\eta} \mathcal{F}(\xi)(p) & =\sum_{r=1}^{n} \partial_{\eta}\left(\rho_{r} \cdot \mathcal{F}_{r}\right)(p) \\
& =\sum_{r=1}^{n}\left(\partial_{\eta} \rho_{r}\right)(p) \cdot \mathcal{F}_{r}(p)^{0}+\rho_{r}(p) \cdot\left(\partial_{\eta} \mathcal{F}_{r}\right)(p) \\
& =\sum_{r=1}^{n} \rho_{r}(p) \cdot\left(\partial_{\eta} \mathcal{F}_{r}\right)(p) .
\end{aligned}
$$

Se $p \in \partial M \backslash U_{r}$, então $\rho_{r}(p)=0$; se $p \in \partial M \cap U_{r}$, então $\left(\partial_{\eta} \mathcal{F}_{r}\right)(p)=\left(\mathrm{d} \mathcal{F}_{r}\right)_{p}\left(\eta_{p}\right)$

$$
\begin{aligned}
& =\left(\mathrm{d} F_{\xi_{r}}\right)_{\varphi_{r}(p)}\left(\mathrm{d} \varphi_{r}\right)_{p}\left(\eta_{p}\right) \\
& =\left(\mathrm{d} F_{\xi_{r}}\right)_{\varphi_{r}(p)}\left(\frac{\partial}{\partial x_{m}}\right) \\
& =\left(\frac{\partial}{\partial x_{m}} F_{\xi_{r}}\right)\left(x_{1}, \ldots, x_{m-1}, 0\right) \\
& =\xi_{r}\left(x_{1}, \ldots, x_{m-1}, 0\right) \\
& =\xi(p)
\end{aligned}
$$

Ainda, $\mathcal{F}$ depende linearmente de $\xi$ e é contínua, pela Observação B.0.3 do Apêndice B. E isto conclui a prova.

Proposição 2.3.7. $A \mathcal{C}^{k, \alpha}$-classe conforme normalizada, $[\bar{g}]^{0}$, é uma subvariedade mergulhada de $[\bar{g}]$.

Demonstração. Dada $\bar{g} \in \mathcal{M}^{k, \alpha}(M)$, seja $\eta_{\bar{g}}$ o campo unitário (voltado para dentro) normal ao bordo $\partial M$. Defina

$$
\begin{aligned}
\mathcal{N}_{\bar{g}}:[\bar{g}] & \longrightarrow \mathcal{C}^{k-1, \alpha}(\partial M) \\
\phi \bar{g} & \longmapsto \partial_{\eta_{\bar{g}}} \phi
\end{aligned}
$$

Então, $\mathcal{N}_{\bar{g}}^{-1}(\{0\})=[\bar{g}]^{0}$ e o diferencial $\left(\mathrm{d} \mathcal{N}_{\bar{g}}\right)_{\phi \bar{g}}: \mathcal{C}^{k, \alpha}(M) \longrightarrow \mathcal{C}^{k-1, \alpha}(\partial M)$ é dado por $\left(\mathrm{d} \mathcal{N}_{\bar{g}}\right)_{\phi \bar{g}}(\psi)=$ $\partial_{\eta_{\bar{g}}} \psi$, para todo $\phi \bar{g} \in[\bar{g}]$ e $\psi \in \mathcal{C}^{k, \alpha}(M)$. Agora, pela Proposição anterior, $\left(\mathrm{d} \mathcal{N}_{\bar{g}}\right)_{\phi \bar{g}}$ admite um inverso limitado à direita para toda $\phi \bar{g} \in[\bar{g}]$. Segue que o diferencial é sobrejetivo e seu núcleo,

$$
\operatorname{ker}\left(\mathrm{d} \mathcal{N}_{\bar{g}}\right)_{\phi \bar{g}}=\left\{\psi \in \mathcal{C}^{k, \alpha}(M): \partial_{\eta_{\bar{g}}} \psi=0\right\},
$$

tem complemento fechado em $\mathcal{C}^{k, \alpha}(M)$. Daí, $[\bar{g}]^{0}$ é uma subvariedade mergulhada de $[\bar{g}]$.

Podemos também combinar ambas características de interesse na mesma classe conforme definindo a $\mathcal{C}^{k, \alpha}$-classe conforme normalizada que consiste de métricas de volume 1

$$
[\bar{g}]_{1}^{0}=\left\{\phi \bar{g}: \phi \in \mathcal{C}_{+}^{k, \alpha}(M), \partial_{\eta_{\bar{g}}} \phi=0, \int_{M} \phi^{\frac{m}{2}} \omega_{\bar{g}}=1\right\} .
$$

Esta é uma subvariedade mergulhada de $\mathcal{M}^{k, \alpha}(M)_{1}$ e uma subvariedade mergulhada de $[\bar{g}]$. Nós podemos expressar $[\bar{g}]_{1}^{0}$ como $[\bar{g}]^{0} \cap \mathcal{M}^{k, \alpha}(M)_{1}$, por exemplo. O espaço tangente correspondente é identificado com

$$
T_{\bar{g}}[\bar{g}]_{1}^{0}=\left\{\psi \in \mathcal{C}^{k, \alpha}(M)^{0}: \int_{M} \psi \omega_{\bar{g}}=0\right\} .
$$

\section{O Funcional de Hilbert-Einstein}

O Funcional de Hilbert-Einstein é o modelo variacional para o problema de Yamabe. Sob este título apresentaremos fatos conhecidos sobre o funcional de Hilbert-Einstein que estão demonstra- 
dos, em detalhes, no Apêndice A.

O funcional de Hilbert-Einstein $F: \mathcal{M}^{k, \alpha}(M) \longrightarrow \mathbb{R}$ dado por

$$
F(\bar{g})=\int_{M} R_{\bar{g}} \omega_{\bar{g}}
$$

é um funcional suave em $\mathcal{M}^{k, \alpha}(M)$ e em $[\bar{g}]$. A primeira variação de $F$ é dada por

$$
\delta F(\bar{g}) h=-\int_{M}\left\langle R i c_{\bar{g}}-\frac{1}{2} R_{\bar{g}} \bar{g}, h\right\rangle_{\bar{g}} \omega_{\bar{g}}-2 \int_{\partial M}\left(\delta H_{\bar{g}}+\frac{1}{2}\left\langle I I_{\bar{g}}, h\right\rangle\right) \sigma_{\bar{g}},
$$

para todo $h \in \Gamma^{k, \alpha}\left(T^{*} M \otimes T^{*} M\right)$.

Para variedades riemannianas compactas (sem bordo) sabemos que os pontos críticos do funcional $F$, restrito a $\mathcal{M}^{k}(M)_{1}$, são as métricas de Einstein de volume 1 em $M$ e, se $F$ está restrito a $\mathcal{C}^{k, \alpha}$-classe conforme de volume $1,[\bar{g}]_{1}$, então os pontos críticos são aquelas métricas conformes a $\bar{g}$, que têm volume unitário e curvatura escalar constante.

Estamos interessados nos pontos críticos da restrição à $\mathcal{C}^{k, \alpha}$-classe conforme normalizada de volume 1 , denotada por $[\bar{g}]_{1}^{0}$, uma vez que estamos lidando com variedades com bordo. Os pontos críticos de $F$ em $[\bar{g}]_{1}^{0}$ são métricas conformes a $\bar{g}$, com volume unitário, curvatura escalar constante e curvatura média do bordo nula.

Se $\bar{g} \in \mathcal{M}^{k, \alpha}(M)_{1}$ é um ponto crítico de $F$ em $[\bar{g}]_{1}^{0}$, então (veja [26]) a segunda variação de $F$ é dada pela forma quadrática.

$$
\delta^{2} F(\bar{g})(\psi)=\frac{(m-2)}{2} \int_{M}\left((m-1) \Delta_{\bar{g}} \psi-R_{\bar{g}} \psi\right) \psi \omega_{\bar{g}}
$$

onde $\psi \in \mathcal{C}^{k, \alpha}(M)^{0}$ e tem integral nula, e $\bar{g}$ é não degenerada, se $R_{\bar{g}}=0$ ou $\frac{R_{\bar{g}}}{m-1}$ não é um autovalor de $\Delta_{\bar{g}}$ com condições de Neumann no bordo.

De fato, note que $(m-1) \Delta_{g}-R_{g}$, como operador de $\mathcal{C}^{k, \alpha}(M)$ em $\mathcal{C}^{k-2, \alpha}$, é de Fredholm de índice zero, e é precisamente por esta razão que consideramos estes espaços de Hölder. Note que este operador leva o subespaço

$$
\left\{\psi \in \mathcal{C}^{k, \alpha}(M): \partial_{\eta_{\bar{g}}} \psi=0, \int_{M} \psi=0\right\}
$$

no subespaço de $\mathcal{C}^{k-2, \alpha}$ consistindo de funções com integral nula. Segue que

$$
(m-1) \Delta_{\bar{g}}-R_{\bar{g}}: T_{\bar{g}}[\bar{g}]_{1} \longrightarrow \mathcal{C}^{k-2, \alpha}(M)
$$

é de Fredholm de índice zero. Então, a forma quadrática $\delta^{2} F(\bar{g})(\psi, \psi)$ é não degenerada se e só se $\operatorname{ker}\left((m-1) \Delta_{g}-R_{g}\right)=\{0\}$, isto é, se e só se $\frac{R_{\bar{g}}}{m-1}=0$ ou $\frac{R_{\bar{g}}}{m-1}$ não é solução do problema de Neumann

$$
\left\{\begin{array}{ll}
\Delta_{\bar{g}} \psi & =\rho \psi \\
\partial_{\eta_{\bar{g}}} \psi & =0
\end{array} .\right.
$$

\subsubsection{Rigidez Local e Bifurcação de Soluções do Problema de Yamabe}

\section{Rigidez Local}

Nesta seção, apresentaremos um resultado obtido em [30], escrito de forma um pouco diferente para melhor se adaptar ao atual contexto. Para maiores detalhes, veja [30, Proposição 3 e Corolário 4]. As provas são essencialmente as mesmas. Começaremos com a seguinte definição.

Definição 2.3.8. Seja $\bar{g} \in \mathcal{M}^{k, \alpha}(M)_{1}$ com curvatura escalar constante $R_{\bar{g}}$ em $M$. Dizemos que $\bar{g}$ é uma métrica nãa degenerada se $R_{\bar{g}}=0$ ou se $\frac{R_{\bar{g}}}{(m-1)}$ não é um autovalor de $\Delta_{\bar{g}}$, com condição 
de Neumann no bordo $\partial_{\eta_{\bar{g}}} f=0$. Em outras palavras, $\frac{R_{\bar{g}}}{(m-1)}$ não é uma solução do problema de autovalor

$$
\left\{\begin{aligned}
\Delta_{\bar{g}} f & =\lambda f, \text { em } M \\
\partial_{\eta_{\bar{g}}} f & =0, \text { em } \partial M
\end{aligned}\right.
$$

Proposição 2.3.9. Seja $\bar{g}_{*} \in \mathcal{M}^{k, \alpha}(M)_{1}$ uma métrica não degenerada de curvatura escalar constante. Então, existe uma vizinhança aberta $U$ de $\bar{g}_{*}$ em $\mathcal{M}^{k, \alpha}(M)_{1}$ tal que o conjunto

$$
S=\left\{\bar{g} \in U: R_{\bar{g}} \text { é constante }\right\},
$$

é uma subvariedade diferenciável mergulhada de $\mathcal{M}^{k, \alpha}(M)_{1}$ que é fortemente transversal às $\mathcal{C}^{k, \alpha_{-}}$ classes conformes normalizadas, $[\bar{g}]^{0}$.

Demonstração. A prova é uma aplicação direta de [30, Proposição 1].

Corolário 2.3.10. Seja $\bar{g}_{*} \in \mathcal{M}^{k, \alpha}(M)_{1}$ uma métrica não degenerada em $M$ com curvatura escalar constante e curvatura média do bordo nula. Então, existe uma vizinhança aberta $U$ de $\bar{g}_{*}$ em $\mathcal{M}^{k, \alpha}(M)_{1}$ tal que toda $\mathcal{C}^{k, \alpha}$-classe conforme normalizada de métricas em $\mathcal{M}^{k, \alpha}(M)_{1}$ tem no máximo uma métrica de curvatura escalar constante e volume 1 em $U$.

Demonstração. O fato de a variedade $S$ ser fortemente transversal às classes conformes normalizadas garante a unicidade local de interseções entre elas.

\section{Bifurcação de Soluções}

Seja $M$ uma variedade riemanniana compacta $m$-dimensional com bordo, $m \geq 3$. Defina

$$
\begin{aligned}
{[a, b] } & \longrightarrow \mathcal{M}^{k, \alpha}(M)_{1}, \quad k \geq 3 \\
s & \longmapsto \bar{g}_{s}
\end{aligned}
$$

um caminho contínuo de métricas riemannianas em $M$ tendo curvatura escalar constante $R_{\bar{g}_{s}}$ e curvatura média do bordo $H_{\bar{g}_{s}}$ nula, para todo $s \in[a, b]$.

Definição 2.3.11. Um instante $s_{*} \in[a, b]$ é chamado um instante de bifurcação para a família de métricas $\left\{\bar{g}_{s}\right\}_{s \in[a, b]}$ se existe uma sequência $\left(s_{n}\right)_{n \geq 1} \subset[a, b]$ e uma sequência $\left(\bar{g}_{n}\right)_{n \geq 1} \subset \mathcal{M}^{k, \alpha}(M)_{1}$ de métricas riemannianas em $M$ satisfazendo:

(a) $\lim _{n \rightarrow \infty} s_{n}=s_{*} e \lim _{n \rightarrow \infty} \bar{g}_{n}=\bar{g}_{s_{*}} \in \mathcal{M}^{k, \alpha}(M)_{1}$;

(b) $\bar{g}_{n} \in\left[\bar{g}_{s_{n}}\right]$, mas $\bar{g}_{n} \neq \bar{g}_{s_{n}}$, para todo $n \geq 1$;

(c) $\bar{g}_{n}$ tem curvatura escalar constante e curvatura média do bordo nula, para todo $n \geq 1$.

Se $s_{*} \in[a, b]$ não é um instante de bifurcação, a famılia $\left\{\bar{g}_{s}\right\}_{s \in[a, b]}$ é dita localmente rígida em $s_{*}$.

Um instante $s \in[a, b]$ para o qual $\frac{R_{\bar{g}_{s}}}{(m-1)}$ é uma solução não nula do problema (2.8) é chamado um instante de degeneração para a família $\left\{g_{s}\right\}_{s \in[a, b]}$. Instantes de degeneração serão candidatos a instantes de bifurcação.

Teorema 2.3.12. Seja $M$ uma variedade riemanniana compacta $m$-dimensional com bordo $\partial M \neq$ $\emptyset, m \geq 3$, e seja

$$
\begin{aligned}
{[a, b] } & \longrightarrow \mathcal{M}^{k, \alpha}(M)_{1}, \quad k \geq 3 \\
s & \longmapsto \bar{g}_{s}
\end{aligned}
$$

um caminho $C^{1}$ de métricas riemannianas em $M$ com curvatura escalar constante $R_{\bar{g}_{s}}$ e curvatura média do bordo $H_{\bar{g}_{s}}=0$. Denote por $n_{s}$ o número de autovalores do operador de Laplace-Beltrami 
$\Delta_{\bar{g}_{s}}$, com condições de Neumann no bordo (contados com multiplicidades), que são menores que $\frac{R_{\bar{g}_{s}}}{(m-1)}$. Assuma que, se $s=a$ ou $s=b, \frac{R_{\bar{g}_{s}}}{(m-1)}=0$ ou não é um autovalor de $\Delta_{\bar{g}_{s}}$, com condiçôes de Neumann no bordo, e $n_{a} \neq n_{b}$. Então, existe um instante de bifurcação $s_{*} \in(a, b)$ para a familia $\left\{\bar{g}_{s}\right\}_{s \in[a, b]}$.

Demonstração. O resultado segue do Teorema de Bifurcação 1.3.2 (não equivariante) para fibrados [31, Teorema A.2, Apêndice A].

Observação 2.3.13. Dada uma variedade riemanniana $(M, \bar{g})$ com bordo mínimo $\partial M \neq \emptyset$, é importante enfatizar que para todo $s>0$, a variedade $(M, s \bar{g})$ também tem bordo minimo, uma vez que $H_{s \bar{g}}=\frac{1}{\sqrt{s}} H_{\bar{g}}$. Além disso, $\Delta_{s \bar{g}}=\frac{1}{s} \Delta_{\bar{g}}, R_{s \bar{g}}=\frac{1}{s} R_{\bar{g}}$ e $\eta_{s \bar{g}}=\frac{1}{\sqrt{s}} \eta_{\bar{g}}$, como demonstramos na Seção 1.5. Isso significa que os sinais dos autovalores no espectro do operador $\Delta_{\bar{g}}-\frac{R_{\bar{g}}}{m-1}$, com condições de Neumann no bordo, são invariantes por homotetias da métrica; de fato, $\lambda$ é autovalor de $\Delta_{\bar{g}}$ se e só se $\frac{\lambda}{s}$ é autovalor de $\Delta_{s \bar{g}}$. Por outro lado, $\omega_{s \bar{g}}=s^{\frac{m}{2}} \omega_{\bar{g}}$, o que altera o volume. Como $R_{\bar{g}}$ constante implica em $R_{s \bar{g}}$ constante para todo $s>0$, tomaremos métricas de volume 1 sempre que necessário sem alterar a teoria espectral do operador $\Delta_{\bar{g}}-\frac{R_{\bar{g}}}{m-1}$.

\subsubsection{Bifurcação de Soluções para o Problema de Yamabe em Variedades Produto com Bordo Mínimo}

Seja $\left(M_{1}, g^{(1)}\right)$ uma variedade riemanniana compacta com $\partial M_{1}=\emptyset$ e curvatura escalar constante, e seja $\left(M_{2}, \bar{g}^{(2)}\right)$ uma variedade riemanniana compacta com bordo mínimo e curvatura escalar constante. Considere a variedade produto, $M=M_{1} \times M_{2}$, cujo bordo é dado por $\partial M=M_{1} \times \partial M_{2}$. Sejam $m_{1}$ e $m_{2}$ as dimensões de $M_{1}$ e $M_{2}$, respectivamente, e assuma que $\operatorname{dim}(M)=m=m_{1}+m_{2} \geq 3$. Para cada $s \in(0,+\infty)$, defina $\bar{g}_{s}=g^{(1)} \oplus s \bar{g}^{(2)}$ uma família de métricas em $M$. Então $\left\{\bar{g}_{s}\right\}_{s} \subset \mathcal{M}^{k, \alpha}(M)$ e valem as afirmações abaixo, que estão demonstradas na Seção 1.5:

(a) $\left(M, \bar{g}_{s}\right)$ tem curvatura escalar constante para todo $s>0$, e sua curvatura escalar é dada por

$$
R_{\bar{g}_{s}}=R_{g^{(1)}}+R_{s \bar{g}^{(2)}}=R_{g^{(1)}}+\frac{1}{s} R_{\bar{g}^{(2)}}
$$

(b) Já que podemos identificar o espaço tangente da variedade produto $T_{(p, q)} M$ com a soma direta $T_{p} M_{1} \oplus T_{q} M_{2}$, para $p \in M_{1}$ e $q \in M_{2}$, o campo vetorial interior $\eta_{s}$, normal a $\partial M$, pode ser escrito como

$$
\eta_{s}=0+\frac{1}{\sqrt{s}} \eta_{2}
$$

onde $\eta_{2}$ é o campo vetorial interior normal a $\partial M_{2}$.

(c) A curvatura média de $\partial M$ é zero, uma vez que

$$
H_{\bar{g}_{s}}=H_{s \bar{g}^{(2)}}=\frac{1}{\sqrt{s}} H_{\bar{g}^{(2)}} .
$$

(d) O operador Laplace-Beltrami, com respeito à $\bar{g}_{s}$, é dado por

$$
\Delta_{\bar{g}_{s}}=\left(\Delta_{g^{(1)}} \otimes I\right)+\frac{1}{s}\left(I \otimes \Delta_{\bar{g}^{(2)}}\right) .
$$

(e) Considerar a família $\left\{\bar{g}_{s}\right\}_{s>0}$ para o propósito de estudar instantes de bifurcação não causa perda de generalidade. De fato, $s_{*}$ é um instante de bifurcação para a família $\left\{g^{(1)} \oplus s \bar{g}^{(2)}\right\}_{s>0}$ se e só se $s_{*}$ é um instante de bifurcação para a família $\left\{\frac{1}{s} g^{(1)} \oplus \bar{g}^{(2)}\right\}_{s>0}$ em $M$. O mesmo é válido para instantes de degeneração. 
Agora, podemos normalizar $\bar{g}_{s}$ para que tenha volume 1, sem perder as informações nas quais estamos interessados, conforme observamos no final da seção anterior; assim, $\left\{\bar{g}_{s}\right\}_{s>0}$ é uma família de pontos críticos do funcional

$$
F: \mathcal{M}^{k, \alpha}(M) \longrightarrow \mathbb{R}
$$

restrito a $[\bar{g}]_{1}^{0}$, isto é, $\left\{\bar{g}_{s}\right\}_{s>0}$ é uma família de métricas com bordo mínimo que são soluções para o problema de Yamabe na variedade produto $M$.

Segundo os resultados apresentados na seção anterior, se $\bar{g}_{s}$ é uma métrica não degenerada, de curvatura escalar constante, curvatura média do bordo nula e volume 1, então (localmente) ela é a única, em sua própria classe conforme, com essas características. Logo, uma condição necessária para que s seja um instante de bifurcação para a família $\left\{\bar{g}_{s}\right\}_{s>0}$ é que $\bar{g}_{s}$ seja degenerada, no sentido discutido na Seção 2.3.1. Note que, $\bar{g}_{s}$ é degenerada se e somente se $\frac{R_{\bar{g}_{s}}}{m-1}$ é autovalor não nulo de $\Delta_{\bar{g}_{s}}$, com condição de Neumann no bordo; o que é equivalente a dizer que zero é um autovalor do operador $\mathcal{J}_{s}$, dado por

$$
\mathcal{J}_{s}=\Delta_{\bar{g}_{s}}-\frac{R_{\bar{g}_{s}}}{m-1}
$$

cujo domínio é $\left\{\psi \in \mathcal{C}^{k, \alpha}(M)^{0}: \int_{M} \psi \omega_{\bar{g}_{s}}=0\right\}$, satisfazendo condições de Neumann no bordo. Portanto, estamos interessados em estudar o espectro do operador $\mathcal{J}_{s}$, que denotaremos por $\Sigma\left(\mathcal{J}_{s}\right)$.

Sejam $0=\rho_{0}^{(1)}<\rho_{1}^{(1)}<\rho_{2}^{(1)}<\ldots$ a sequência de todos os autovalores distintos de $\Delta_{g^{(1)}}$, com multiplicidade geométrica $\mu_{i}^{(1)}, i \geq 0$, e $0=\rho_{0}^{(2)}<\rho_{1}^{(2)}<\rho_{2}^{(2)}<\ldots$ a sequência de todos os autovalores distintos de $\Delta_{\bar{g}^{(2)}}$, que resolvem o problema de autovalor com condição de Neumann,

$$
\left\{\begin{aligned}
\Delta_{\bar{g}^{(2)}} f^{(2)} & =\rho_{j}^{(2)} f^{(2)}, \text { on } M \\
\partial_{\eta_{2}} f^{(2)} & =0, \text { on } \partial M
\end{aligned}\right.
$$

onde $\mu_{j}^{(2)}$ é a multiplicidade geométrica de $\rho_{j}^{(2)}, j \geq 0$. Então, o espectro de $\mathcal{J}_{s}$ é dado por

$$
\Sigma\left(\mathcal{J}_{s}\right)=\left\{\sigma_{i, j}: i, j \geq 0, i+j>0\right\}
$$

onde

$$
\sigma_{i, j}(s)=\rho_{i}^{(1)}+\frac{1}{s} \rho_{j}^{(2)}-\frac{1}{m-1}\left(R_{g^{(1)}}+\frac{1}{s} R_{\bar{g}^{(2)}}\right)
$$

são os autovalores de $\mathcal{J}_{s}$, com condição de Neumann no bordo $\partial M$, com multiplicidade geométrica igual ao produto $\mu_{i}^{(1)} \mu_{j}^{(2)}$. Segue do conteúdo apresentado na Seção 1.5.7 que todos os autovalores de $\mathcal{J}_{s}$ são da forma $\sigma_{i, j}(s)$. Além disso, vale a pena verificar que $\sigma_{i, j}(s)$ é solução do problema de Neumann,

$$
\left\{\begin{aligned}
\mathcal{J}_{s} f & =\sigma_{i, j}(s) f, \text { on } M \\
\partial_{\eta_{s}} f & =0, \text { on } \partial M
\end{aligned}\right.
$$

Sejam $f_{i}^{(1)}$ uma autofunção de $\Delta_{g^{(1)}}$ associada ao autovalor $\rho_{i}^{(1)}$ e $f_{j}^{(2)}$ uma autofunção associada a $\rho_{j}^{(2)}$, solução do problema (2.9), então $f=f_{i}^{(1)} \otimes f_{j}^{(2)}$ é autofunção de $\mathcal{J}_{s}$, associada ao autovalor 
$\sigma_{i, j}(s)$ e satisfaz (2.10). De fato,

$$
\begin{aligned}
\mathcal{J}_{s} f & =\left(\Delta_{\bar{g}_{s}}-\frac{R_{\bar{g}_{s}}}{m-1}\right) f \\
& =\Delta_{g^{(1)}} \otimes I+\frac{1}{s}\left(I \otimes \Delta_{\bar{g}}^{(2)}\right)\left(f_{i}^{(1)} \otimes f_{j}^{(2)}\right)-\left(\frac{R_{\bar{g}_{s}}}{m-1}\right)\left(f_{i}^{(1)} \otimes f_{j}^{(2)}\right) \\
& =\Delta_{g^{(1)}} f_{i}^{(1)} \otimes f_{j}^{(2)}+\frac{1}{s}\left(f_{i}^{(1)} \otimes \Delta_{\bar{g}}^{(2)} f_{j}^{(2)}\right)-\left(\frac{R_{\bar{g}_{s}}}{m-1}\right)\left(f_{i}^{(1)} \otimes f_{j}^{(2)}\right) \\
& =\rho_{i}^{(1)} f_{i}^{(1)} \otimes f_{j}^{(2)}+\frac{1}{s} f_{i}^{(1)} \otimes\left(\rho_{j}^{(2)} f_{j}^{(2)}\right)-\left(\frac{R_{\bar{g}_{s}}}{m-1}\right)\left(f_{i}^{(1)} \otimes f_{j}^{(2)}\right) \\
& =\rho_{i}^{(1)}\left(f_{i}^{(1)} \otimes f_{j}^{(2)}\right)+\frac{1}{s} \rho_{j}^{(2)}\left(f_{i}^{(1)} \otimes f_{j}^{(2)}\right)-\frac{1}{m-1}\left(R_{g^{(1)}}+\frac{1}{s} R_{\bar{g}^{(2)}}\right)\left(f_{i}^{(1)} \otimes f_{j}^{(2)}\right) \\
& =\left(\rho_{i}^{(1)}+\frac{1}{s} \rho_{j}^{(2)}\right)\left(f_{i}^{(1)} \otimes f_{j}^{(2)}\right)-\frac{1}{m-1}\left(R_{g^{(1)}}+\frac{1}{s} R_{\bar{g}^{(2)}}\right)\left(f_{i}^{(1)} \otimes f_{j}^{(2)}\right) \\
& =\left[\rho_{i}^{(1)}+\frac{1}{s} \rho_{j}^{(2)}-\frac{1}{m-1}\left(R_{g^{(1)}}+\frac{1}{s} R_{\bar{g}^{(2)}}\right)\right]\left(f_{i}^{(1)} \otimes f_{j}^{(2)}\right) \\
& =\sigma_{i, j}(s) f
\end{aligned}
$$

e, temos

$$
\begin{aligned}
\partial_{\eta_{s}} f & =\eta_{s}\left(f_{i}^{(1)} \otimes f_{j}^{(2)}\right)=\left(0+\frac{1}{\sqrt{s}} \eta_{\bar{g}^{(2)}}\right)\left(f_{i}^{(1)} \otimes f_{j}^{(2)}\right) \\
& =\frac{1}{\sqrt{s}} \eta_{\bar{g}^{(2)}} f_{j}^{(2)}=\frac{1}{\sqrt{s}} \partial_{\eta_{s}} f_{j}^{(2)}=0
\end{aligned}
$$

Embora os autovalores $\rho_{i}^{(1)}$, de $\Delta_{g^{(1)}}$ e $\rho_{j}^{(2)}$, de $\Delta_{\bar{g}^{(2)}}$ sejam todos distintos, os autovalores $\sigma_{i, j}(s)$ de $\mathcal{J}_{s}$ não são necessariamente distintos.

Acabamos de comentar que, se $\sigma_{i, j}(s)=0$, a métrica $\bar{g}_{s}$ é degenerada e $s$ pode ser um instante de bifurcação, mas devemos excluir o caso em que $\sigma_{i, j}(s)$ é identicamente nula, caso em que a degenerescência de $\bar{g}_{s}$ não depende de $s$, mas ocorre pelo fato de $g^{(1)}$ e $\bar{g}^{(2)}$ serem ambas degeneradas. Assim, é importante considerarmos a seguinte definição.

Definição 2.3.14. Sejam $i_{*}$ e $j_{*}$ os menores inteiros não negativos que satisfazem

$$
\rho_{i_{*}}^{(1)} \geq \frac{R_{g^{(1)}}}{m-1}, \quad \rho_{j_{*}}^{(2)} \geq \frac{R_{\bar{g}^{(2)}}}{m-1} .
$$

Dizemos que o par $\left(g^{(1)}, \bar{g}^{(2)}\right)$ é degenerado se valem as igualdades em ambos os casos, isto é, $\sigma_{i_{*}, j_{*}}(s)=0$, para todo $s$. Nesta situação, o operador $\mathcal{J}_{s}$ é também chamado degenerado.

Podemos afirmar que, se $R_{g^{(1)}}<0$ ou se $R_{\bar{g}^{(2)}}<0$ e $H_{\bar{g}^{(2)}}=0$, então $\left(g^{(1)}, \bar{g}^{(2)}\right)$ é, certamente, não degenerado, dado que os autovalores $\rho_{i}^{(1)}, \rho_{j}^{(2)}$ são todos positivos. Observe também que, se o par $\left(g^{(1)}, \bar{g}^{(2)}\right)$ é degenerado, zero é um autovalor de $\mathcal{J}_{s}$, para todo $s \in(0,+\infty)$, caso contrário, existe apenas um conjunto discreto, enumerável, $S \subset(0,+\infty)$, de instantes $s$ para os quais o operador $\mathcal{J}_{s}$ é singular. Primeiro, consideramos o caso em que ambas as curvaturas são positivas.

\section{O Caso da Curvatura Escalar Positiva}

Estamos interessados em estudar os zeros da função $s \mapsto \sigma_{i, j}(s)$, a medida que $i, j$ variam. De imediato, podemos tirar algumas conclusões; por exemplo, se a função $\sigma_{i, j}$ não é identicamente 
nula, para $i, j$ fixos, ela tem no máximo um zero em $(0,+\infty)$. Vamos escrever $\sigma$ como

$$
\sigma_{i, j}(s)=\rho_{i}^{(1)}-\frac{R_{g^{(1)}}}{m-1}+\frac{1}{s}\left(\rho_{j}^{(2)}-\frac{R_{\bar{g}^{(2)}}}{m-1}\right) .
$$

Derivando, temos

$$
\sigma_{i, j}^{\prime}(s)=-\frac{1}{s^{2}}\left(\rho_{j}^{(2)}-\frac{R_{\bar{g}^{(2)}}}{m-1}\right)
$$

então, $\sigma_{i, j}^{\prime}(s)=0$ se e somente se $\rho_{j}^{(2)}=\frac{R_{\bar{g}^{(2)}}}{m-1}\left(\right.$ caso em que $\rho_{j}^{(2)}$ é uma solução de $\left.(2.9)\right)$. Se $\sigma_{i, j}^{\prime}(s)=0$ para algum $s$, então $\sigma_{i, j}^{\prime}(s)=0$ para todo $s \in(0,+\infty)$. Portanto, $\sigma$ é estritamente monótona ou constante, assim, se $\sigma_{i, j}$ não é identicamente nula (constante), certamente tem no máximo um zero (estritamente monótona).

Lema 2.3.15. Assuma que o par $\left(g^{(1)}, \bar{g}^{(2)}\right)$ seja não degenerado e que $R_{g^{(1)}}, R_{\bar{g}^{(2)}}>0$, com $H_{\bar{g}^{(2)}}=0$. Então, as funções $\sigma_{i, j}(s)$ satisfazem as seguintes propriedades:

(a.) Para todo $i, j \geq 0$, a aplicação $s \mapsto \sigma_{i, j}(s)$ é estritamente monótona em $(0,+\infty)$, exceto aquelas $\sigma_{i, j_{*}}$ que são constantes iguais a $\rho_{i}^{(1)}-\frac{R_{g^{(1)}}}{m-1}$, quando $\rho_{j_{*}}^{(2)}=\frac{R_{\bar{g}^{(2)}}}{m-1}$.

(b.) Para $i \neq i_{*}$ e $j \neq j_{*}$, a aplicação $\sigma_{i, j}(s)$ admite um zero se e só se:

- $j<j_{*}$ e $i>i_{*}$, caso em que $\sigma_{i, j}$ é estritamente crescente,

- $j>j_{*}$ e $i<i_{*}$, caso em que $\sigma_{i, j}$ é estritamente decrescente.

(c.) $S e \rho_{i_{*}}^{(1)}=\frac{R_{g^{(1)}}}{m-1}$, então $\sigma_{i_{*}, j}$ não tem zeros, qualquer que seja $j \in(0,+\infty)$. Se $\rho_{i_{*}}^{(1)}>\frac{R_{g^{(1)}}}{m-1}$, então $\sigma_{i_{*}, j}$ tem um zero se e somente se $j<j_{*}$.

(d.) $S e \rho_{j_{*}}^{(2)}=\frac{R_{\bar{g}}^{(2)}}{m-1}$, então $\sigma_{i, j_{*}}$ não tem zeros, qualquer que seja $i \in(0,+\infty)$. Se $\rho_{j_{*}}^{(2)}>\frac{R_{\bar{g}^{(2)}}}{m-1}$, então $\sigma_{i, j_{*}}$ tem um zero se e só se $i<i_{*}$.

Demonstração. O resultado segue diretamente da análise da expressão

$$
\sigma_{i, j}(s)=\left(\rho_{i}^{(1)}-\frac{R_{g^{(1)}}}{m-1}\right)+\frac{1}{s}\left(\rho_{j}^{(2)}-\frac{R_{\bar{g}^{(2)}}}{m-1}\right) .
$$

Corolário 2.3.16. Se o par $\left(g^{(1)}, \bar{g}^{(2)}\right)$ é não degenerado, então o conjunto $S \subset(0,+\infty)$, de instantes s para os quais $\mathcal{J}_{s}$ é singular, é enumerável e discreto. Mais precisamente, consiste de uma sequência estritamente decrescente $\left(s_{n}^{(1)}\right)_{n}$ tendendo a 0 e de uma sequência estritamente crescente, ilimitada $\left(s_{n}^{(2)}\right)_{n}$. Para todos os demais valores de $s, \mathcal{J}_{s}$ é um isomorfismo, em particular, a família $\left\{\bar{g}_{s}\right\}_{s>0}$ é localmente rígida nesses instantes.

Demonstração. Pelo Lema 2.3.15, cada função $\sigma_{i, j}$ tem no máximo um zero, portanto existe apenas uma quantidade enumerável de instantes de degeneração para $\mathcal{J}_{s}$. Seja $s_{i j}$ o zero de $\sigma_{i j}$, então

$$
0<s_{i j}=-\frac{\rho_{j}^{(2)}-\frac{R_{\bar{g}^{(2)}}}{m-1}}{\rho_{i}^{(1)}-\frac{R_{g^{(1)}}}{m-1}} .
$$

Agora, vamos estudar o comportamento desses zeros em dois casos: 
- se $j>j_{*}$ e $i<i_{*}$, quando $j \rightarrow+\infty$, temos

$$
s_{i j}=\frac{\rho_{j}^{(2)}-\frac{R_{\bar{g}^{(2)}}}{m-1}}{\frac{R_{g^{(1)}}}{m-1}-\rho_{i}^{(1)}} \geq\left(\rho_{j}^{(2)}-\frac{R_{\bar{g}^{(2)}}}{m-1}\right) \frac{1}{\frac{R_{g^{(1)}}-\rho_{i_{*}-1}^{(1)}}{m-1}} \rightarrow+\infty .
$$

- se $i>i_{*}$ e $j<j_{*}$, quando $i \rightarrow+\infty$, temos

$$
s_{i j}=\frac{\frac{R_{\bar{g}^{(2)}}}{m-1}-\rho_{j}^{(2)}}{\rho_{i}^{(1)}-\frac{R_{g^{(1)}}}{m-1}} \leq \frac{1}{\rho_{i}^{(1)}-\frac{R_{g^{(1)}}}{m-1}}\left(\frac{R_{\bar{g}^{(2)}}}{m-1}-\rho_{1}^{(2)}\right) \rightarrow 0 .
$$

Portanto, o conjunto $S$, de todos os zeros das funções $\sigma_{i, j}$, tem pontos de acumulação apenas em 0 e $+\infty$. Seja $s_{*} \in(0,+\infty) \backslash S$. Então, $\mathcal{J}_{s_{*}}$ é um isomorfismo, isto é, $0 \notin \Sigma\left(J_{s *}\right)$ ou, equivalentemente, $\frac{R_{\bar{g}_{s_{*}}}}{m-1}$ não é um autovalor de $\Delta_{\bar{g}_{s_{*}}}$ (e $R_{\bar{g}_{s_{*}}}$ é obviamente diferente de zero, uma vez que estamos considerando apenas curvatura escalar positiva). Então, $\bar{g}_{s_{*}} \in\left\{\bar{g}_{s}\right\}_{s>0}$ é uma métrica não degenerada. Segue da Proposição 2.3.9 que a família $\left\{\bar{g}_{s}\right\}_{s>0}$ é localmente rígida em $s_{*}$.

Teorema 2.3.17. Seja $\left(M_{1}, g^{(1)}\right)$ uma variedade riemanniana compacta com curvatura escalar constante e seja $\left(M_{2}, \bar{g}^{(2)}\right)$ uma variedade riemanniana compacta com bordo, curvatura escalar constante e bordo minimo $\partial M_{2}$. Assuma que o par $\left(g^{(1)}, \bar{g}^{(2)}\right)$ é não degenerado. Para todo $s \in$ $(0,+\infty)$, seja $\bar{g}_{s}=g^{(1)} \oplus s \bar{g}^{(2)}$ uma métrica na variedade produto com bordo, $M=M_{1} \times M_{2}$. Então, existe uma sequência tendendo a 0 e uma sequência tendendo a $+\infty$ consistindo de instantes de bifurcação para a familia $\left\{\bar{g}_{s}\right\}_{s>0}$.

Demonstração. Vamos mostrar agora que os instantes de bifurcação consistem de duas subsequências das sequências $\left(s_{n}^{(1)}\right)_{n}$ e $\left(s_{n}^{(2)}\right)_{n}$, de instantes onde $\mathcal{J}_{s}$ é singular, cuja existência provamos no Corolário 2.3.16. Respeitando a notação já utilizada, seja $n_{0}>0$ tal que $s_{n}^{(1)}<s_{1}^{(2)}$ e $s_{1}^{(1)}<s_{n}^{(2)}$, para todo $n>n_{0}$. Então, existe um $\varepsilon>0$, para todo $n>n_{0}$, tal que o operador $\mathcal{J}_{s}$ é um isomorfismo nos intervalos $\left[s_{n}^{(1)}-\varepsilon, s_{n}^{(1)}+\varepsilon\right]$ e $\left[s_{n}^{(2)}-\varepsilon, s_{n}^{(2)}+\varepsilon\right]$, exceto pelos instantes $s_{n}^{(1)}$ e $s_{n}^{(2)}$, propriamente ditos.

Uma vez que os zeros das funções crescentes se acumulam em 0 e os zeros das funções decrescentes se acumulam em $+\infty$, se $\sigma_{p, q}$ é uma função não-crescente, para todo $s \in\left(0, s_{n}^{(1)}+\varepsilon\right]$, $n>n_{0}$, temos $\sigma_{p, q}(s) \neq 0$. Então, $\sigma_{p, q}\left(s_{n}^{(1)}-\varepsilon\right)<0$ se e somente se $\sigma_{p, q}\left(s_{n}^{(1)}+\varepsilon\right)<0$. Por outro lado, se $\sigma_{i, j}$ é uma função crescente, para todo $n>n_{0}$, temos $\sigma_{i, j}\left(s_{n}^{(1)}\right)=0, \sigma_{i, j}\left(s_{n}^{(1)}-\varepsilon\right)<0$, $\sigma_{i, j}\left(s_{n}^{(1)}+\varepsilon\right)>0$ e, o fato de $s_{n}^{(1)}<s_{1}^{(2)}$ assegura que não existe função decrescente que tenha zero em $s_{n}^{(1)}$. Portanto, podemos concluir com certeza que $n_{s_{n}^{(1)}-\varepsilon} \neq n_{s_{n}^{(1)}+\varepsilon}$. Pelo Teorema 2.3.12, segue que a subsequência $\left(s_{n}^{(1)}\right)_{n>n_{0}}$ é a sequência de instantes de bifurcação, tendendo a 0 que estávamos procurando.

Agora, analisando o comportamento das funções não-decrescentes e das funções decrescentes nos instantes $s_{n}^{(2)}$, para $n>n_{0}$, de maneira análoga, obtemos $n_{s_{n}}^{(2)}-\varepsilon \neq n_{s_{n}}^{(2)}+\varepsilon$ e podemos aplicar o Teorema 3.3 para concluir que a subsequência $\left(s_{n}^{(2)}\right)_{n>n_{0}}$ é a sequência de instantes de bifurcação, tendendo a $+\infty$ que procurávamos.

Quando temos um par degenerado, não podemos aplicar o Teorema 2.3.12, pois, neste caso, para todo $s \in(0,+\infty)$, o zero é um autovalor de $\mathcal{J}_{s}$, e portanto as hipóteses do teorema nunca são satisfeitas. É interessante notar que os instantes de degeneração que sobram entre $s_{1}^{(1)}$ e $s_{1}^{(2)}$ ficam indecidíveis e não podem ser tratados pelo o Teorema 2.3.17, chamaremos estes de instantes 
neutros e utilizaremos uma abordagem diferente, no Capítulo 3, para estudar se, em algum deles, ocorre bifurcação.

\section{O Caso de Curvatura Escalar Não-Positiva}

Agora, considere a família $\left\{\bar{g}_{s}\right\}_{s>0}$ na variedade produto $M=M_{1} \times M_{2}$ no caso em que uma ou ambas as curvaturas $R_{g^{(1)}}$ or $R_{\bar{g}^{(2)}}$, são não-positivas, mantendo as condições de Neumann no bordo $\partial M_{2}$. Observe que, se $R_{g^{(1)}}$ e $R_{\bar{g}^{(2)}}$ são ambas não-positivas, o par $\left(g^{(1)}, \bar{g}^{(2)}\right)$ é não-degenerado.

Se $R_{g^{(1)}} \leq 0$ e $R_{\bar{g}^{(2)}}>0$, então o par $\left(g^{(1)}, \bar{g}^{(2)}\right)$ é degenerado se e só se $R_{g^{(1)}}=0$ e $\rho_{j_{*}}^{(2)}=\frac{R_{\bar{g}^{(2)}}}{m-1}$, quando $\rho_{i}^{(1)} \geq 0, \forall i \in\{0,1,2, \ldots\}$.

Teorema 2.3.18. As seguintes afirmações são verdadeiras:

(a) Se $R_{g^{(1)}} \leq 0$ e $R_{\bar{g}^{(2)}} \leq 0$, então a familia $\left\{\bar{g}_{s}\right\}_{s>0}$ não tem instantes de degeneração, e, portanto, é localmente rígida em todo $s \in(0,+\infty)$.

(b) Se $R_{g^{(1)}} \leq 0, R_{\bar{g}^{(2)}}>0$, e o par $\left(g^{(1)}, \bar{g}^{(2)}\right)$ é não-degenerado, então o conjunto de instantes de degeneração para $\mathcal{J}_{s}$ consiste de uma sequência estritamente decrescente $\left(s_{n}\right)_{n \in \mathbb{N}}$ que converge para 0 quando $n \longrightarrow \infty$. Ademais, todo instante de degeneração é um instante de bifurcação para a família $\left\{\bar{g}_{s}\right\}_{s>0}$

(c) Simetricamente, se $R_{g^{(1)}}>0, R_{\bar{g}^{(2)}} \leq 0$, e o par $\left(g^{(1)}, \bar{g}^{(2)}\right)$ é não-degenerado, então o conjunto dos instantes de degeneração para $\mathcal{J}_{\text {s }}$ consiste de uma sequência não-limitada, estritamente crescente $\left(s_{n}\right)_{n \in \mathbb{N}}$ e todo instante de degeneração é um instante de bifurcação para a familia $\left\{\bar{g}_{s}\right\}_{s>0}$.

Demonstração. O resultado segue da análise das funções:

$$
\sigma_{i, j}(s)=\rho_{i}^{(1)}+\frac{1}{s} \rho_{j}^{(2)}-\frac{1}{m-1}\left(R_{g^{(1)}}+\frac{1}{s} R_{\bar{g}^{(2)}}\right) .
$$

No caso (a), é fácil ver que $\sigma_{i, j}(s)>0$, para todo $i, j=0,1,2, \ldots$, com $i+j>0$, então $\mathcal{J}_{s}$ não tem autovalores nulos e o resultado segue.

No caso (b), as funções $\sigma_{i, j}$ admitem um zero apenas se $i \geq 0$ e $j<j_{*}$. Se $s_{i, j}$ denota o zero de uma tal função, temos

$$
0<s_{i, j}=\left|\frac{\rho_{j}^{(2)}-\frac{R_{\bar{g}^{(2)}}}{m-1}}{\rho_{i}^{(1)}-\frac{R_{g^{(1)}}}{m-1}}\right| \leq \frac{R_{\bar{g}^{(2)}}}{\rho_{i}^{(1)}-\frac{R_{g^{(1)}}}{m-1}} \longrightarrow 0, \text { as } i \longrightarrow+\infty
$$

Portanto, existe uma sequência decrescente $\left(s_{n}\right)_{n \in \mathbb{N}}$ de instantes de degeneração que acumulam no 0 . Uma vez que $\left(s_{n}\right)_{n \in \mathbb{N}}$ acumula apenas no zero, para cada $n \in \mathbb{N}$, existe $\varepsilon>0$ tal que o intervalo $\left[s_{n}-\varepsilon, s_{n}+\varepsilon\right]$ tem apenas $s_{n}$ como instante de degeneração. Argumentando como na prova do Teorema 2.3.17, temos $n_{s_{n}-\varepsilon} \neq n_{s_{n}+\varepsilon}$. A conclusão segue do Teorema 2.3.12.

Procedendo de maneira similar no caso (c), as funções $\sigma_{i, j}$ admitem um zero apenas se $i<i^{*}$ e $j \geq 0$, e temos uma sequência crescente, não-limitada, de instantes de degeneração, cada um dos quais é um instante de bifurcação para a família $\left\{\bar{g}_{s}\right\}_{s>0}$. 


\section{Capítulo 3}

\section{Bifurcação Equivariante de Soluções para Problema de Yamabe}

No capítulo anterior, vimos resultados de bifurcação para uma família de soluções do problema de Yamabe em variedades produto compactas. Lima, Piccione e Zedda, em [31], estudaram o caso sem considerar condições de bordo e nós estudamos, em [14], bifurcação de uma família de soluções para o problema de Yamabe em variedades produto compactas com bordo mínimo. Em ambos os casos percebemos que, para uma quantidade finita de instantes de degeneração $s_{1}^{(1)}<s<s_{1}^{(2)}$, aos quais chamamos instantes neutros, o Teorema 2.3.12 não foi suficiente para decidirmos se ocorria bifurcação.

Em [31], Lima, Piccione e Zedda, consideraram a ação (por isometrias) de um grupo de Lie conexo em um dos fatores da variedade produto $M$. Assumindo o que chamaram de ação harmonicamente livre e usando uma noção de representações essencialmente equivalentes, aplicaram o Teorema de Bifurcação Equivariante 1.3.3 para garantir que a bifurcação ocorre nos instantes neutros.

Neste capítulo, consideraremos a ação de um grupo de Lie conexo na variedade produto com bordo mínimo. Apresentaremos uma definição diferente de ação harmonicamente livre (Definição 3.2.1), que usaremos como hipótese para demonstrar um novo resultado de bifurcação (equivariante) de soluções para o problema de Yamabe em variedades produto com bordo mínimo, o principal resultado (Proposição 3.2.6) deste capítulo. A ação harmonicamente livre definida neste capítulo será suficiente para garantir que as representações do grupo $G$ nos autoespaços negativos do operador $\mathcal{J}_{s}$ em extremos de intervalos que contenham instantes neutros são não-equivalentes, de modo que poderemos utilizar o mesmo Teorema de Bifurcação Equivariante 1.3.3, usado em [31], para garantir a ocorrência de bifurcação também nos instantes neutros.

\subsection{Bifurcação Equivariante em Variedades com Bordo Mí- nimo}

Assuma conhecidos todos os fatos provados na Seção 2.3 .1 e considere $M$ uma variedade compacta com bordo $\partial M \neq 0$, de dimensão $m \geq 3$. Seja

$$
\begin{aligned}
{[a, b] } & \longrightarrow \mathcal{M}^{k, \alpha}(M)_{1}, \quad k \geq 3 \\
s & \longmapsto \bar{g}_{s}
\end{aligned}
$$

um caminho contínuo de métricas riemannianas em $M$ (de volume 1) tendo curvatura escalar constante $R_{\bar{g}_{s}}$ e curvatura média do bordo $H_{\bar{g}_{s}}$ nula, para todo $s \in[a, b]$.

Seja $G$ um grupo de Lie conexo, de dimensão finita, agindo em $M$ por difeomorfismos que 
preservam orientação e a métrica $\bar{g}_{s}$, para todo $s \in[a, b]$, isto é,

$$
\begin{aligned}
\xi: G \times M & \longrightarrow M \\
(g, x) & \longmapsto g \cdot x,
\end{aligned}
$$

é uma ação de grupo

$$
\begin{aligned}
& \xi(e, x)=e \cdot x=x \\
& \xi\left(g_{1}, \xi\left(g_{2}, x\right)\right)=\xi\left(g_{1} g_{2}, x\right)
\end{aligned}
$$

onde $e$ é o elemento neutro em $G$ e $G$ é um grupo de Lie tal que

$$
G \subset \bigcap_{s \in[a, b]} I s o\left(M, \bar{g}_{s}\right)
$$

Portanto,

$$
\begin{aligned}
\xi_{g}: \begin{array}{l}
M \\
x
\end{array} & \longmapsto \xi_{g}(x)=g \cdot x \quad \text { é uma isometria em } M, \text { para todo } g \in G, \mathrm{e} \\
\xi^{x} \quad: \quad \begin{array}{l}
G \\
g
\end{array} & \longmapsto \xi^{x}(g)=g \cdot x \quad \text { é diferenciável, para todo } x \in M .
\end{aligned}
$$

Notação: Embora estejamos usando notações diferentes para g, elemento do grupo $G$ e $\bar{g}$, métrica riemanniana na variedade com bordo, daqui por diante, escreveremos $\xi(x)=\xi \cdot x$ para indicar a ação de $G, \xi(g, x)=\xi_{g}(x)=g \cdot x$.

Seja

$$
\Delta_{\bar{g}_{s}}: T_{\bar{g}_{s}}\left[\bar{g}_{s}\right]_{1}^{0} \longrightarrow \mathcal{C}^{k-2, \alpha}(M),
$$

o operador laplaciano da variedade $\left(M, \bar{g}_{s}\right)$ restrito a

$$
T_{\bar{g}_{s}}\left[\bar{g}_{s}\right]_{1}^{0}=\left\{\psi \in \mathcal{C}^{k, \alpha}(M): \partial_{\eta_{s}} \psi=0 \text { e } \int_{M} \psi \omega_{\bar{g}_{s}}=0\right\}
$$

Observe que, se $f \in T_{\bar{g}_{s}}\left[\bar{g}_{s}\right]_{1}^{0}$, então, para toda isometria $\xi \in G$, temos

$$
\int_{M}(f \circ \xi) \omega_{\bar{g}_{s}}=\int_{M}(f \circ \xi) \xi^{*}\left(\omega_{\bar{g}_{s}}\right)=\int_{M} \xi^{*}\left(f \omega_{\bar{g}_{s}}\right)=\int_{M} f \omega_{\bar{g}_{s}}=0
$$

além disso, como já mostramos na Proposição 3.1.1, $\partial_{\eta_{s}}(f \circ \xi)=0$, logo $f \circ \xi \in T_{\bar{g}_{s}}\left[\bar{g}_{s}\right]_{1}^{0}$.

Lembre que $\Delta_{\bar{g}_{s}}:=-d i v_{\bar{g}_{s}} \nabla$ tem infinitos autovalores não-negativos e as dimensões dos autoespaços associados são todas finitas. Agora, para cada autovalor $\rho$ de $\Delta_{\bar{g}_{s}}$, solução do problema de Neumann

$$
\left\{\begin{aligned}
\Delta_{\bar{g}_{s}} f & =\rho f, \text { on } M \\
\partial_{\eta_{s}} f & =0, \text { on } \partial M
\end{aligned}\right.
$$

onde $\eta_{s}$ é o campo unitário (interior) normal ao bordo de $M$, denote por $V_{s, \rho} \subset T_{\bar{g}_{s}}\left[\bar{g}_{s}\right]_{1}^{0}$ o autoespaço correspondente.

Proposição 3.1.1. Para cada $\rho$, solução de 3.2, a aplicação linear

$$
\pi_{s, \rho}: G \longrightarrow G L\left(V_{s, \rho}\right)
$$


definida por

$$
\begin{aligned}
\pi_{s, \rho}(\xi): V_{s, \rho} & \longrightarrow V_{s, \rho} \\
f & \mapsto f \circ \xi
\end{aligned}
$$

é uma antirrepresentação de $G$ em $V_{s, \rho}$.

Demonstração. O resultado segue de (3.1) e dos resultados da Seção 3.1, onde provamos que os autoespaços do laplaciano são invariantes por isometrias (Proposição 3.1.1) e que a ação isométrica de $G$ em $M$ determina antirrepresentações de $G$ em todos os autoespaços do laplaciano (Proposição 3.1 .2 .

Vimos que uma métrica $\bar{g}_{s}$, ponto crítico do funcional de Hilbert-Einstein restrito à classe conforme normalizada de volume $1,[\bar{g}]^{0}$, é não-degenerada se e só se $R_{\bar{g}_{s}}=0$ ou $\frac{R_{\bar{g}_{s}}}{m-1}$ não é autovalor do operador $\Delta_{\bar{g}_{s}}$, com condições de Neumann no bordo. O resultado de rigidez 2.3.9, garante unicidade local de solução para o problema de Yamabe se $\bar{g}_{s}$ é não-degenerada. De modo que, os instantes de bifurcação, conforme Definição 2.3.11, devem ocorrer dentre os instantes de degeneração. Assim, se queremos estabelecer condições para que haja bifurcação, é de nosso interesse estudarmos o espectro do laplaciano, ou equivalentemente, o espectro do operador

$$
\mathcal{J}_{s}=\Delta_{\bar{g}_{s}}-\frac{R_{\bar{g}_{s}}}{m-1} I
$$

onde $I$ denota o operador identidade. Como os autovalores do laplaciano são todos positivos e $0=\rho_{0}<\rho_{1}<\ldots<\rho_{t}<\ldots$, existe uma quantidade finita de autovalores $\rho \leq \frac{R_{\bar{g}_{s}}}{m-1}$, ou seja, existe uma quantidade finita de autovalores negativos para o operador $\mathcal{J}_{s}$.

Assim, para cada $s \in[a, b]$, podemos definir a antirrepresentação de $G$ no autoespaço negativo de $\mathcal{J}_{s}$, considerando a representação soma direta,

$$
\begin{aligned}
\pi_{s}^{-}: G & \longrightarrow G L\left(V_{s}^{-}\right) \\
\xi & \longmapsto \pi_{s}^{-}(\xi)
\end{aligned}
$$

onde

$$
\pi_{s}^{-}=\bigoplus_{\rho \leq \frac{R_{\bar{g}_{s}}}{m-1}} \pi_{s, \rho} \quad \text { e } \quad V_{s}^{-}=\bigoplus_{\rho \leq \frac{R_{\bar{g}_{s}}}{m-1}} V_{s, \rho}
$$

e a transformação linear

$$
\begin{aligned}
\pi_{s}^{-}(\xi): V_{s}^{-} & \longrightarrow V_{s}^{-} \\
f & \longmapsto \pi_{s}^{-}(\xi) f
\end{aligned}
$$

é dada por

$$
\pi_{s}^{-}(\xi) f=\pi_{s, \rho_{0}}(\xi) f_{0} \oplus \ldots \oplus \pi_{s, \rho_{r}}(\xi) f_{r},
$$

com $f=f_{0} \oplus \ldots \oplus f_{r} \in V_{s}^{-}$, onde $f_{i} \in V_{s, \rho_{i}}$, para todo $i=0, \ldots, r$.

Então, o seguinte resultado estabelece condições de bifurcação de soluções para o problema de Yamabe em variedades com bordo. Este teorema de bifurcação equivariante para variedades com bordo mínimo é uma extensão natural do [31, Teorema 3.4]. Transcrevemos a demonstração, detalhando sua validade para o caso com bordo.

Teorema 3.1.2. Na situação acima descrita, suponha que a família $\left\{\bar{g}_{s}\right\}_{s \in[a, b]}$ é localmente rígida nos extremos do intervalo $[a, b]$ e que as representações $\pi_{a}^{-}$e $\pi_{b}^{-}$são não-equivalentes. Então, existe um instante de bifurcação $s_{*}$ para a família $\left\{\bar{g}_{s}\right\}_{s \in[a, b]}$ no intervalo $(a, b)$.

Demonstração. Sejam $B_{0}=\mathcal{C}^{k-2, \alpha}(M), B_{2}=\mathcal{C}_{+}^{k, \alpha}(M)$ espaços de Banach e $\mathcal{H}=L^{2}(M)$ o espaço das funções $L^{2}$ integráveis com respeito à medida induzida pela forma volume em $M$ associada à qualquer das métricas $\bar{g}_{s}$. Considere a ação de $G$ sobre esses espaços via pull-back e defina os 
fibrados vetoriais

$$
\mathcal{D}^{0}=\left\{(\phi, s) \in \mathcal{C}_{+}^{k, \alpha}(M) \times[a, b]: \int_{M} \phi^{\frac{m}{2}} \omega_{\bar{g}_{s}}=1, \partial_{\eta_{s}} \phi=0\right\},
$$

e

$$
\mathcal{E}^{0}=\left\{(\psi, s) \in \mathcal{C}^{k-2, \alpha}(M) \times[a, b]: \int_{M} \psi \omega_{\bar{g}_{s}}=0, \partial_{\eta_{s}} \psi=0\right\},
$$

sobre o intervalo $[a, b]$, onde $\eta_{s}$ é o campo unitário (interior) normal ao bordo de $M$, com respeito à métrica $\bar{g}_{s}$.

O conjunto

$$
\mathcal{E}_{s}^{0}=\left\{\psi \in \mathcal{C}^{k-2, \alpha}(M): \int_{M} \psi \omega_{\bar{g}_{s}}=0, \partial_{\eta_{s}} \psi=0\right\}
$$

é fechado em $\mathcal{C}^{k-2, \alpha}(M)$ e contém a função nula, logo é um subespaço de Banach de $B_{0}$. Em particular, $\mathcal{E}^{0}$ é subfibrado do fibrado trivial $\mathcal{C}^{k-2, \alpha}(M) \times[a, b]$, donde $s \mapsto \mathcal{E}_{s}^{0}$ é uma família $\mathcal{C}^{1}$ de subespaços fechados do espaço de Banach $B_{0}$. Observe que $G$ age também sobre $\mathcal{M}^{k, \alpha}(M)_{1}$ via pull-back: para cada isometria $\xi$ e cada métrica $\tilde{g} \in \mathcal{M}^{k, \alpha}(M)_{1}$, temos $\xi^{*} \tilde{g}=\tilde{g}$. Sendo assim, para toda $\psi \in \mathcal{E}_{s}^{0}$, temos

$$
\int_{M} \psi \circ \xi \omega_{\bar{g}_{s}}=\int_{M} \xi^{*}\left(\psi \omega_{\bar{g}_{s}}\right)=\int_{M} \psi \omega_{\bar{g}_{s}}=0
$$

e

$$
\partial_{\eta_{s}}(\xi \circ \psi)=\partial_{\eta_{s}} \xi(\psi) \cdot \partial_{\eta_{s}} \psi=0
$$

portanto, $\mathcal{E}_{s}^{0}$ é $G$-invariante.

De forma análoga, definimos

$$
\mathcal{D}_{s}^{0}=\left\{\phi \in \mathcal{C}_{+}^{k, \alpha}(M): \int_{M} \phi^{\frac{m}{2}} \omega_{\bar{g}_{s}}=1, \partial_{\eta_{s}} \phi=0\right\} .
$$

Observe que, para cada $s, \mathcal{D}_{s}^{0}$ pode ser identificado com $\left[\bar{g}_{s}\right]_{1}^{0}$ que, como vimos na Seção 2.3.1, é subvariedade mergulhada de $\left[\bar{g}_{s}\right]$, que pode ser identificada com $B_{2}$. Logo, $\mathcal{D}_{s}^{0}$ é subvariedade de $B_{2}$. Como $\mathcal{D}^{0}$ é subfibrado do fibrado trivial $B_{2} \times[a, b]$, segue que $\mathcal{D}_{s}^{0}$ é uma família $\mathcal{C}^{1}$ de subvariedades de $B_{2}$. A ação de $G$ deixa $\mathcal{D}^{0}$ invariante, pois para toda $\phi \in \mathcal{D}_{s}^{0}, \xi \in G$, temos $\partial_{\eta_{s}}(\xi \circ \psi)=0, \mathrm{e}$

$$
\int_{M}(\phi \circ \xi)^{\frac{m}{2}} \omega_{\bar{g}_{s}}=\int_{M}\left(\phi^{\frac{m}{2}} \circ \xi\right) \omega_{\bar{g}_{s}}=\int_{M} \xi^{*}\left(\phi^{\frac{m}{2}} \omega_{\bar{g}_{s}}\right)=\int_{M} \phi^{\frac{m}{2}} \omega_{\bar{g}_{s}}=1 .
$$

Defina, ainda,

$$
\mathcal{H}_{s}=\left\{\kappa \in L^{2}(M): \int_{M} \kappa \omega_{\bar{g}_{s}}=0\right\}
$$

subespaço fechado de $\mathcal{H}$ e note que, para cada $s \in[a, b]$ existe um produto interno completo, dado por

$$
\left\langle\kappa_{1}, \kappa_{2}\right\rangle_{s}=\int_{M} \kappa_{1} \cdot \kappa_{2} \omega_{\bar{g}_{s}},
$$

para todo $\kappa_{1}, \kappa_{2} \in \mathcal{H}_{s}$. De forma análoga ao que fizemos para $\mathcal{E}_{s}^{0}$, mostra-se que $\mathcal{H}_{s}$ é $G$-invariante.

Agora, definimos a aplicação

$$
\begin{aligned}
F: \mathcal{D}^{0} & \longrightarrow \mathcal{E}^{0} \\
(\phi, s) & \longmapsto\left(R_{\phi \bar{g}_{s}}-\int_{M} R_{\phi \bar{g}_{s}} \omega_{\bar{g}_{s}}, s\right) ;
\end{aligned}
$$


que é um morfismo de fibrados: veja que o diagrama $\mathcal{D}^{0} \longrightarrow \frac{F}{\pi_{\mathcal{D}^{0}}} \mathcal{E}^{0}{ }_{\pi_{\mathcal{E}^{0}}}$ é comutativo.

Note que $s \mapsto \phi_{s} \in \mathcal{D}_{s}^{0}$ é uma seção $\mathcal{C}^{1}$ do fibrado $\mathcal{D}^{0}$ e $s \mapsto \psi_{s} \in \mathcal{E}_{s}^{0}$ é uma seção $\mathcal{C}^{1}$ do fibrado $\mathcal{E}^{0}$, com $F(\phi, s)=(\psi, s)$, se $(\phi, s) \in \mathcal{D}^{0}$, a métrica $\phi \bar{g}_{s} \in\left[\bar{g}_{s}\right]$ tem curvatura escalar constante, se e só se $F(\phi, s)=(\mathbf{0}, s)=\mathbf{0}_{s}$, onde, para cada $s, \mathbf{0}_{s}$ é a função nula em $\mathcal{E}_{s}^{0}$. Logo, estamos interessados na imagem inversa, por $F$, da seção nula do fibrado $\mathcal{E}^{0}$, conjunto que denotamos por

$$
F^{-1}\left(\mathbf{0}_{s}\right)=\left\{(\phi, s) \in \mathcal{D}^{0}: F(\phi, s)=(\mathbf{0}, s)\right\} .
$$

Observe que a seção constante

$$
\begin{aligned}
\mathbb{1}_{\mathcal{D}^{0}}:[a, b] & \longrightarrow \mathcal{D}_{s}^{0} \\
s & \longmapsto \mathbb{1}_{\mathcal{D}^{0}}(s)=\mathbf{1}_{s},
\end{aligned}
$$

que associa a cada $s \in[a, b]$ a função constante igual a 1 , denotada por $\mathbf{1}_{s}: M \rightarrow \mathbb{R}$, em $\mathcal{D}_{s}^{0}$, pertence a $F^{-1}\left(\mathbf{0}_{s}\right)$, já que, por hipótese, $\bar{g}_{s}$ tem curvatura escalar constante, para todo $s \in[a, b]$. As órbitas de $\mathbf{1}_{s}$ e $\mathbf{0}_{s}$ pela ação de $G$ em $\mathcal{E}_{s}^{0}$ e $\mathcal{D}_{s}^{0}$ (induzida pela ação de $G$ em $B_{0}$ e $B_{2}$ ) são triviais, $\mathbf{1}_{s} \cdot \xi=\mathbf{1}_{s}$ e $\mathbf{0}_{s} \circ \xi=\mathbf{0}_{s}$, isto é, $G$ fixa as órbitas de $\mathbf{1}_{s}$ e de $\mathbf{0}_{s}$. O espaço tangente à $\mathcal{D}_{s}^{0}$ em $\mathbf{1}_{s}$ é dado por

$$
T_{\mathbf{1}_{s}} \mathcal{D}_{s}^{0}=\left\{\psi \in \mathcal{C}^{k, \alpha}(M): \int_{M} \psi \omega_{\bar{g}_{s}}=0, \partial_{\eta_{s}} \psi=0\right\}
$$

Lembre que

daí, as inclusões

$$
\mathcal{C}^{k, \alpha}(M)^{0}=\left\{\psi \in \mathcal{C}^{k, \alpha}(M): \partial_{\eta_{s}} \psi=0\right\}
$$

$$
\mathcal{C}^{k, \alpha}(M)^{0} \subset \mathcal{C}^{k, \alpha}(M) \subset \mathcal{C}^{k-2, \alpha}(M) \subset L^{2}(M)
$$

induzem as inclusões

$$
T_{\mathbf{1}_{s}} \mathcal{D}_{s}^{0} \subset \mathcal{E}_{s}^{0} \subset \mathcal{H}_{s}
$$

para todo $s$ no intervalo $[a, b]$.

Vamos mostrar que $F_{s}=F(\cdot, s): \mathcal{D}_{s}^{0} \rightarrow \mathcal{E}_{s}^{0}$ é um operador gradiente para todo $s \in[a, b]$, isto é, que o diferencial $\left(d F_{s}\right)_{1_{s}}: T_{\mathbf{1}_{s}} \mathcal{D}_{s}^{0} \rightarrow \mathcal{E}_{s}^{0}$ é simétrico com respeito ao produto interno completo em $\mathcal{H}_{s}$ para cada $s \in[a, b]$. Com efeito, para cada $\psi \in T_{\mathbf{1}_{s}} \mathcal{D}_{s}^{0},\left(d F_{s}\right)_{\mathbf{1}_{s}}$ é dado por

$$
\begin{aligned}
\left(d F_{s}\right)_{\mathbf{1}_{s}}(\psi) & =\left.\frac{d}{d t}\right|_{t=0} F_{s}\left(\mathbf{1}_{s}+t \psi\right) \\
& =\left.\frac{d}{d t}\right|_{t=0}\left(R_{\left(\mathbf{1}_{s}+t \psi\right) \bar{g}_{s}}-\int_{M} R_{\left(\mathbf{1}_{s}+t \psi\right) \bar{g}_{s}} \omega_{\bar{g}_{s}}\right) \\
& =\left.\frac{d}{d t}\right|_{t=0}\left(R_{\left(\bar{g}_{s}+t \psi \bar{g}_{s}\right)}-\int_{M} R_{\left(\bar{g}_{s}+t \psi \bar{g}_{s}\right)} \omega_{\bar{g}_{s}}\right)
\end{aligned}
$$

Observe que $\bar{g}_{s}+t \psi \bar{g}_{s}=\bar{g}_{s}(t)$ é uma variação de $\bar{g}_{s}$ na direção do tensor $h_{s}=\psi \bar{g}_{s} \in T_{\bar{g}}[\bar{g}]_{1}^{0}$. 
Assim, como fizemos nas contas do Apêndice A, temos

$$
\begin{aligned}
\left.\frac{d}{d t}\right|_{t=0} R_{\bar{g}_{s}(t)} & =-\psi \bar{g}_{s}^{i j} R_{i j}+\nabla_{i}\left(\nabla_{j} \psi \bar{g}_{s}^{i j}-\nabla^{i}(m \psi)\right) \\
& =-\psi \bar{g}_{s}^{i j} R_{i j}+\nabla_{i}\left(\nabla^{i} \psi-m \nabla^{i} \psi\right) \\
& =-\psi R_{\bar{g}_{s}}-\Delta_{\bar{g}_{s}} \psi+m \Delta_{\bar{g}_{s}} \psi \\
& =(m-1) \Delta_{\bar{g}_{s}} \psi-\psi R_{\bar{g}_{s}}
\end{aligned}
$$

onde estamos considerando o laplaciano geométrico, $\Delta \psi=-\nabla_{i} \nabla^{i} \psi$, e utilizando a notação de Einstein; daí

$$
\begin{aligned}
\left.\frac{d}{d t}\right|_{t=0} \int_{M} R_{\bar{g}_{s}(t)} \omega_{\bar{g}_{s}} & =\int_{M}\left((m-1) \Delta_{\bar{g}_{s}} \psi-\psi R_{\bar{g}_{s}}\right) \omega_{\bar{g}_{s}} \\
& =(m-1) \int_{M} \Delta_{\bar{g}_{s}} \psi \omega_{\bar{g}_{s}}-\underbrace{R_{\bar{g}_{s}}}_{c t e} \underbrace{\int_{M} \psi \omega_{\bar{g}_{s}}}_{=0} \\
& =(m-1) \int_{\partial M}\left\langle\nabla \psi, \eta_{s}\right\rangle \omega_{\bar{g}_{s}} \\
& =(m-1) \int_{\partial M} \partial_{\eta_{s}} \psi \omega_{\bar{g}_{s}}=0
\end{aligned}
$$

Portanto, $\left(d F_{s}\right)_{\mathbf{1}_{s}}(\psi)=(m-1) \Delta_{\bar{g}_{s}} \psi-R_{\bar{g}_{s}} \psi$, que também pode ser escrito como

$$
\left(d F_{s}\right)_{\mathbf{1}_{s}}(\psi)=(m-1)\left(\Delta_{\bar{g}_{s}} \psi-\frac{R_{\bar{g}_{s}}}{m-1} \psi\right) .
$$

Agora, o operador linear $\mathcal{J}_{s}=\Delta_{\bar{g}_{s}}-\frac{R_{\bar{g}_{s}}}{m-1} I$, definido em $T_{\mathbf{1}_{s}} \mathcal{D}_{s}^{0}$, é simétrico com respeito ao produto interno $\langle\cdot, \cdot\rangle_{s}$. De fato, se $\psi, \varphi \in T_{\mathbf{1}_{s}} \mathcal{D}_{s}^{0}$, então

$$
\begin{aligned}
\left\langle\mathcal{J}_{s} \psi, \varphi\right\rangle_{s} & =\int_{M}\left(\Delta_{\bar{g}_{s}} \psi-\frac{R_{\bar{g}_{s}}}{m-1} \psi\right) \varphi \omega_{\bar{g}_{s}} \\
& =\int_{M}\left(\Delta_{\bar{g}_{s}} \psi\right) \varphi \omega_{\bar{g}_{s}}-\int_{M}\left(\frac{R_{\bar{g}_{s}}}{m-1}\right) \varphi \omega_{\bar{g}_{s}} \\
& =\int_{M}\left(-\nabla^{i}\left(\nabla_{i}\right) \psi\right) \varphi \omega_{\bar{g}_{s}}-\int_{M} \frac{R_{\bar{g}_{s}}}{m-1} \psi \omega_{\bar{g}_{s}} \\
& =\int_{M} \psi\left(-\nabla^{i}\left(\nabla_{i}\right) \varphi\right) \omega_{\bar{g}_{s}}-\int_{M} \psi\left(\frac{R \bar{g}_{s}}{m-1} \varphi\right) \omega_{\bar{g}_{s}} \\
& =\int_{M} \psi\left(\Delta_{\bar{g}_{s}} \varphi\right) \omega_{\bar{g}_{s}}-\int_{M} \psi\left(\frac{R_{\bar{g}_{s}}}{m-1} \varphi\right) \varphi \omega_{\bar{g}_{s}} \\
& =\int_{M} \psi\left(\Delta_{\bar{g}_{s}} \varphi-\frac{R_{\bar{g}_{s}}}{m-1} \varphi\right) \omega_{\bar{g}_{s}} \\
& =\left\langle\psi, \mathcal{J}_{s} \varphi\right\rangle_{s} .
\end{aligned}
$$

Logo, $F_{s}$ é operador gradiente, como queríamos.

Também, a aplicação $F$ é $G$-equivariante, isto é,

$$
F(\phi \cdot \xi, s)=F(\phi, s) \cdot \xi
$$

ou, equivalentemente, $F_{s}(\phi \cdot \xi)=F_{s}(\phi) \cdot \xi$, para todo $(\phi, s) \in \mathcal{D}^{0}$. Verdadeiramente, por um lado, 
temos

$$
\begin{aligned}
F_{s}(\phi \cdot \xi) & =F_{s}(\phi \circ \xi) \\
& =R_{(\phi \circ \xi) \bar{g}_{s}}-\int_{M} R_{(\phi \circ \xi) \bar{g}_{s}} \omega_{\bar{g}_{s}}
\end{aligned}
$$

por outro lado, $F_{s}(\phi) \cdot \xi=F_{s}(\phi) \circ \xi=\xi^{*} F_{s}(\phi)$. Decorre de $R_{(\phi \circ \xi) \bar{g}_{s}}=R_{\phi \bar{g}_{s}} \circ \xi$ (vide Apêndice C) que

$$
F_{s}(\phi \cdot \xi)=R_{(\phi \circ \xi) \bar{g}_{s}}-\int_{M} R_{(\phi \circ \xi) \bar{g}_{s}} \omega_{\bar{g}_{s}}=\underbrace{R_{\phi \bar{g}_{s}} \circ \xi}_{\xi^{*} R_{\phi \bar{g}_{s}}}-\int_{M} \underbrace{R_{\phi \bar{g}_{s}} \circ \xi \omega_{\bar{g}_{s}}}_{\xi^{*}\left(R_{\phi \bar{g}_{s}} \omega_{\bar{g}_{s}}\right)}=F_{s}(\phi) \cdot \xi,
$$

donde segue que $F$ é $G$-equivariante.

Observe que

$$
\left(d F_{s}\right)_{\mathbf{1}_{s}}(\psi)=(m-1) \Delta_{\bar{g}_{s}} \psi-R_{\bar{g}_{s}} \psi,
$$

leva $T_{\mathbf{1}_{s}} \mathcal{D}_{s}^{0}$ em $\mathcal{E}_{s}^{0}$. De fato, $\partial_{\eta_{s}}\left((m-1) \Delta_{\bar{g}_{s}} \psi-\psi R_{\bar{g}_{s}}\right)=0$, para toda $\psi \in T_{\mathbf{1}_{s}} \mathcal{D}_{s}^{0}$, e,

$$
\begin{aligned}
\int_{M}(m-1) \Delta_{\bar{g}_{s}} \psi-R_{\bar{g}_{s}} \psi \omega_{\bar{g}_{s}} & =(m-1) \int_{M} \Delta_{\bar{g}_{s}} \psi \omega_{\bar{g}_{s}}-\underbrace{R_{\bar{g}_{s}}}_{c t e} \underbrace{\int_{M} \psi \omega_{\bar{g}_{s}}}_{=0} \\
& =(m-1) \int_{\partial M} \underbrace{\left\langle\nabla \psi, \eta_{s}\right\rangle}_{\partial_{\eta_{s}} \psi=0} \omega_{\bar{g}_{s}}=0 .
\end{aligned}
$$

Afirmamos que o operador

$$
\left(d F_{s}\right)_{\mathbf{1}_{s}}: T_{\mathbf{1}_{s}} \mathcal{D}_{s}^{0} \longrightarrow \mathcal{E}_{s}^{0}
$$

é de Fredholm de índice zero. Com efeito, é um fato clássico, conhecido da análise, que o operador laplaciano $\Delta_{\bar{g}}$ é de Fredholm de índice zero quando considerado como operador do espaço $\mathcal{C}^{k, \alpha}(M)$

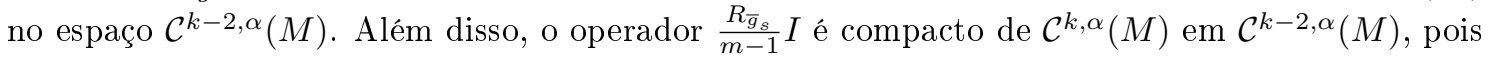
o fato de $k+\alpha>k-2+\alpha$ implica que a inclusão $i: \mathcal{C}^{k, \alpha}(M) \hookrightarrow \mathcal{C}^{k-2, \alpha}(M)$ é compacta (como demonstrado na Proposição 2.2.9). Agora, como $T_{\mathbf{1}_{s}} \mathcal{D}_{s}$ é subespaço fechado de $\mathcal{C}^{k, \alpha}(M)$, $\mathcal{E}_{s}$ é subespaço fechado de $\mathcal{C}^{k-2, \alpha}(M)$, e

$$
T_{\mathbf{1}} \mathcal{D}_{s} \stackrel{i}{\longrightarrow} \mathcal{C}^{k, \alpha}(M) \stackrel{\Delta_{\bar{g}_{s}}+\frac{R_{\bar{g}_{s}}}{m-1}}{\longrightarrow} \mathcal{C}^{k-2, \alpha}(M) \stackrel{p}{\longrightarrow} \mathcal{E}_{s}
$$

onde

- $\operatorname{dim} \operatorname{ker}(i)=0, \operatorname{codim} \operatorname{im}(i)=1 \Longrightarrow$ ind $(i)=-1$,

- $\operatorname{dim} \operatorname{ker}(p)=1, \operatorname{codim} \operatorname{im}(p)=0 \Longrightarrow$ ind $(p)=1$,

segue que $\Delta_{\bar{g}_{s}}+\frac{R_{\bar{g}_{s}}}{m-1}: T_{\mathbf{1}_{s}} \mathcal{D}_{s} \longrightarrow \mathcal{E}_{s}$ é de Fredholm de índice zero, como queríamos.

Sabemos que existe uma base ortonormal $\beta_{s}$ de autofunções do laplaciano $\Delta_{\bar{g}_{s}}$ para o espaço de Hilbert $L^{2}\left(M, \bar{g}_{s}\right)$ com respeito ao produto interno (3.3), além disso, $\mathcal{H}_{s}$ é um subespaço fechado de $L^{2}\left(M, \bar{g}_{s}\right)$ que é ortogonal ao subespaço, de dimensão 1, das funções constantes:

$$
L^{2}\left(M, \bar{g}_{s}\right)=\mathcal{H}_{s} \oplus\left\{\iota \in L^{2}\left(M, \bar{g}_{s}\right): \iota \text { é constante }\right\} .
$$

O subespaço das funções constantes é gerado pela autofunção constante igual a 1 associada ao autovalor nulo do laplaciano, de modo que $\beta_{s} \backslash\{\mathbf{1}\}$ é uma base de autofunções do laplaciano para $\mathcal{H}_{s}$. Observe que, se $\lambda$ é um autovalor de $\Delta_{\bar{g}_{s}}$, com condição de Neumann no bordo, então $\lambda-\frac{R_{\bar{g}_{s}}}{m-1}$ é autovalor de $\Delta_{\bar{g}_{s}}-\frac{R_{\bar{g}_{s}}}{m-1}$ e $f$ é autofunção de $\Delta_{\bar{g}_{s}}$ associada ao autovalor $\lambda$ se e somente se $f$ é 
autofunção de $\Delta_{\bar{g}_{s}}-\frac{R_{\bar{g}_{s}}}{m-1}$ associada ao autovalor $\lambda-\frac{R_{\bar{g}_{s}}}{m-1}$. Em particular, 1 é autofunção associada ao autovalor $-\frac{R_{\bar{g}_{s}}}{m-1}$ do operador $\mathcal{J}_{s}$. Portanto, $\beta_{s} \backslash \mathbf{1}$ é uma base de autofunções de $\mathcal{J}_{s}$ para $\mathcal{H}_{s}$.

Como os autovalores de $\Delta_{\bar{g}_{s}}$ são todos reais, não-negativos, de multiplicidade finita e o espectro de $\mathcal{J}_{s}$ é dado por

$$
\Sigma\left(\mathcal{J}_{s}\right)=\left\{\lambda-\frac{R_{\bar{g}_{s}}}{m-1}: \lambda \in \Sigma\left(\Delta_{\bar{g}_{s}}\right)\right\},
$$

segue que $\mathcal{J}_{s}$ tem um número finito de autovalores negativos, o que equivale a dizer que $\left(\mathrm{d} F_{s}\right)_{\mathbf{1}_{s}}$ tem um número finito de autovalores negativos.

Já mostramos que os autoespaços $V_{\rho, s}$ do laplaciano $\Delta_{\bar{g}_{s}}$ são $G$-invariantes. É fácil ver que, $V_{\rho, s}$ como autoespaços de $\mathcal{J}_{s}: T_{\mathbf{1}} \mathcal{D}_{s}^{0} \longrightarrow \mathcal{E}_{s}^{0}$ ou, equivalentemente, $\left(\mathrm{d} F_{s}\right)_{\mathbf{1}_{s}}$ são igualmente $G$ invariantes.

Veja que $\left(\mathrm{d} F_{a}\right)_{\mathbf{1}_{a}}$ é um isomorfismo de $T_{\mathbf{1}_{s}} \mathcal{D}_{s}^{0}$ em $\mathcal{E}_{s}^{0}$ pois, por hipótese, $R_{g_{a}}=0$ ou $\frac{R_{g_{a}}}{m-1}$ não é autovalor de $\Delta_{g_{a}}$, o que implica que $\left(\mathrm{d} F_{a}\right)_{\mathbf{1}_{a}}$ é injetor. Ora, já vimos que $\left(\mathrm{d} F_{a}\right)_{\mathbf{1}_{a}}$ é de Fredholm de índice zero, portanto injetividade implica sobrejetividade. $\mathrm{O}$ mesmo vale para $\left(\mathrm{d} F_{b}\right)_{\mathbf{1}_{b}}$.

Por hipótese, $\pi_{a}^{-}$e $\pi_{b}^{-}$são não-equivalentes. Sob estas condições, o Teorema 1.3.3, garante a existência de um instante de bifurcação $s_{*} \in(a, b)$ para a família de soluções, $s \mapsto \phi_{s} \in \mathcal{D}_{s}^{0}$, da equação

$$
F(\cdot, s)=\left(\mathbf{0}_{s}, s\right) .
$$

Isto significa que $s_{*} \mapsto \phi_{s_{*}} \in \mathcal{D}_{s_{*}}^{0}$ pertence à família de soluções da equação; além disso, a existência do instante de bifurcação $s_{*}$, implica na existência de

(a.) uma sequência $s_{n} \in[a, b]$, com $s_{n} \rightarrow s_{*}$; isto é, existe uma sequência de funções $\phi_{s_{n}} \in \mathcal{D}_{s_{n}}^{0}$ que são soluções da equação (3.5), tal que $\phi_{s_{n}} \rightarrow \phi_{s_{*}}$;

(b.) uma sequência de soluções de (3.5) com $\phi_{n} \in \mathcal{D}_{s_{n}}^{0}, \phi_{n} \rightarrow \phi_{s_{*}}$ e $\phi_{n} \neq \phi_{s_{n}}$, para todo $n \in \mathbb{N}$.

O que é equivalente a dizer que $s_{*}$ é um instante de bifurcação para a família de soluções do problema de Yamabe em variedades com bordo mínimo $s \mapsto \bar{g}_{s} \in\left[\bar{g}_{s}\right]_{1}^{0}$, no sentido de 2.3.11.

\subsection{Bifurcação Equivariante de Soluções do Problema de Ya- mabe em Variedades Produto com Bordo Mínimo}

Seja $\left(M_{1}, g^{(1)}\right)$ uma variedade riemanniana compacta, com $\partial M_{1}=\emptyset$ e curvatura escalar constante, e seja $\left(M_{2}, \bar{g}^{(2)}\right)$ uma variedade riemanniana compacta com bordo mínimo e curvatura escalar constante. Considere a variedade produto, $M=M_{1} \times M_{2}$, cujo bordo é dado por $\partial M=M_{1} \times \partial M_{2}$. Sejam $m_{1}$ e $m_{2}$ as dimensões de $M_{1}$ e $M_{2}$, respectivamente, e assuma que $\operatorname{dim}(M)=m=m_{1}+m_{2} \geq 3$. Para cada $s \in(0,+\infty)$, defina $\bar{g}_{s}=g^{(1)} \oplus s \bar{g}^{(2)}$ uma família de métricas em $M$. Então $\left\{\bar{g}_{s}\right\}_{s} \subset \mathcal{M}^{k, \alpha}(M)$.

$\mathrm{Na}$ Seção 2.3.3, estudamos os instantes de degeneração do operador $\mathcal{J}_{s}$ e provamos que, exceto por uma quantidade finita desses instantes, chamados instantes neutros, cada instante de degeneração é um instante de bifurcação.

Usando o último teorema provado na seção anterior, podemos estabelecer bifurcação em todos os instantes de degeneração do operador $\mathcal{J}_{s}$, inclusive nos instantes neutros. Lembre que chamamos de instante neutro, todo $\bar{s}$ que pode ser zero de autovalores crescentes e decrescentes de $\mathcal{J}_{s}$, não sendo possível portanto, garantir um salto no índice de Morse do operador $\mathcal{J}_{s}$ nos extremos do intervalo $(\bar{s}-\varepsilon, \bar{s}+\varepsilon)$, para todo $\varepsilon>0$ tal que $\bar{s}-\varepsilon$ e $\bar{s}+\varepsilon$ são instantes onde o operador $\mathcal{J}_{(}$. ) é não singular. Isto é, as dimensões dos autoespaços negativos, $V_{\bar{s}-\varepsilon}^{-}$e $V_{\bar{s}+\varepsilon}^{-}$, podem ser iguais, assim, queremos fazer uma análise mais fina, considerando a ação de um grupo de Lie, $G$, sobre a variedade produto $M$ e verificando se as representações de $G$ nesses autoespaços são não-equivalentes.

A construção discutida na Seção 3.1, nos permite apresentar a seguinte definição. 
Definição 3.2.1. Seja $G$ um grupo de Lie que age por isometrias em uma variedade riemanniana $(N, h)$. A ação de $G$ é dita harmonicamente livre se, dada uma família arbitrária de autoespaços do laplaciano $\Delta_{h}$, dois a dois distintos,

$$
V_{1}, V_{2}, \ldots, V_{r}, V_{r+1}, \ldots, V_{r+s}, \quad r, s \geq 1
$$

e dados inteiros $n_{\ell} \geq 0$, com $\ell=1, \ldots, r+s$, não todos nulos, então as antirrepresentações

$$
\bigoplus_{\ell=1}^{r} n_{\ell} \cdot \pi_{\ell} \quad e \quad \bigoplus_{\ell=r+1}^{r+s} n_{\ell} \cdot \pi_{\ell}
$$

são não-equivalentes. Aqui, denotamos por $\pi_{\ell}$ a antirrepresentação de $G$ em $V_{\ell}$, definida na Proposição 3.1.2, e $n_{\ell} \cdot \pi_{\ell}$ denota a soma direta (externa) de $n_{\ell}$ cópias de $\pi_{\ell}$.

Exemplo 3.2.2. Vejamos o exemplo da esfera $\mathbb{S}^{n}$ mergulhada no $\mathbb{R}^{n+1}$. O grupo de isometrias de $\mathbb{R}^{n+1}$ é o grupo ortogonal $O(n+1)$. Considere $\mathbb{S}^{n}$ com a métrica induzida pela métrica euclidiana do $\mathbb{R}^{n+1}$, então, o subgrupo $S O(n+1)$, de $O(n+1)$, age em $\mathbb{S}^{n}$ por isometrias; por outro lado, qualquer isometria de $\mathbb{S}^{n}$ pode ser estendida para uma isometria do $\mathbb{R}^{n+1}$, de modo que $O(n+1)$ é o grupo de isometrias da esfera de dimensão $n$. A ação de $O(n+1)$ na esfera é transitiva (o que implica que $\mathbb{S}^{n}$ é homogênea). De fato, dado $p \in \mathbb{S}^{n}$, obtemos uma base ortonormal $\beta=\left\{v_{1}, v_{2}, \ldots, v_{n}, v_{n+1}\right\}$, do $\mathbb{R}^{n+1}$, que contém o vetor posição $v_{n+1}$ correspondente ao ponto $p$. Agora, a matriz $P \in O(n+1)$, cujas colunas são os vetores da base $\beta$ leva o ponto $e_{n+1}=(0, \ldots, 0,1) \in \mathbb{S}^{n}$ no ponto $p$. Portanto, dados quaisquer dois pontos $p, q \in \mathbb{S}^{n}$, temos $P Q^{-1} q=p$, onde $Q$ é a matriz que leva $e_{n+1}$ em $q$, construída de forma análoga à construção de $P$, o que prova que a ação de $O(n+1)$ é transitiva. Na verdade, podemos considerar a ação de $G_{0}=S O(n+1)$ em $\mathbb{S}^{n}$, que também é transitiva. Agora, o subgrupo de isotropia de $p \in \mathbb{S}^{n}$, denotado por $G_{0}(p)$, é isomorfo a $S O(n) \times\{1\}$. Com efeito, note que o subgrupo de isotropia de $e_{n+1}$ é dado por

$$
G_{0}\left(e_{n+1}\right)=\left\{\bar{A}=\left(\begin{array}{cc}
A & 0 \\
O & 1
\end{array}\right): A \in S O(n), O \text { é a matriz nula } n \times n\right\},
$$

que é isomorfo a $S O(n) \times\{1\}$; para um $p$ qualquer em $\mathbb{S}^{n}$, basta tomar as matrizes $P \bar{A} P^{-1}$, onde $P$ é a matriz construída anteriormente. A prova de que $G_{0}(p)$ age transitivamente nos vetores unitários de $T_{p} \mathbb{S}^{n}$ é similar à prova da transitividade de $O(n+1)$ sobre a esfera, que fizemos acima. Assim, $\mathbb{S}^{n}$ é isotrópica em todo $p$, além disso, $\mathbb{S}^{n}$ é difeomorfa a $S O(n+1) / S O(n)$. As representações de $S O(n+1)$ nos autoespaços do laplaciano de $\mathbb{S}^{n}$ são irredutíveis (isso é consequência de um resultado conhecido, apresentado logo abaixo, como Proposição 3.2.5). Os autovalores do laplaciano de $\mathbb{S}^{n}, n \geq 2$, são conhecidos e dados por $\lambda=k(k+n-1)$, com $k=0,1,2$, .. e as dimensões dos autoespaços correspondentes, dadas por $\operatorname{dim} V_{\lambda}=\left(\begin{array}{c}n+k \\ n\end{array}\right)-\left(\begin{array}{c}n+(k-2) \\ n\end{array}\right)$, formam uma sequência estritamente crescente [36, Cap. III, §22], portanto os autoespaços são não equivalentes ${ }^{1}$. Concluímos que a ação de $S O(n+1)$ sobre $\mathbb{S}^{n}$ é um exemplo de ação harmonicamente livre.

Podemos generalizar o exemplo da esfera, como segue.

Exemplo 3.2.3. Seja $(N, h)$ é uma variedade riemanniana compacta. Quando os autoespaços do laplaciano $\Delta_{h}$ são irredutíveis e dois a dois não equivalentes (conforme definições na Seção 3), a ação do grupo de isometrias $I s o(N, h)$ em $N$ é harmonicamente livre.

Exemplo 3.2.4. O seguinte resultado ([4, Proposição C.I.8]), permite que exibamos uma classe importante de exemplos de ações harmonicamente livres.

Proposição 3.2.5. Sejam $G$ um grupo de Lie (conexo) compacto, $H$ um subgrupo fechado de $G$ e $M=G / H$ o espaço homogêneo correspondente, com uma métrica riemanniana g, que é $G$ invariante. Seja $\Delta_{g}$ o laplaciano de $g$ em $M$ e denote por $V_{\lambda}$, os autoespaços de $\Delta_{g}$ associados

\footnotetext{
${ }^{1}$ Para o caso $n=1$, é sabido que as representações do laplaciano de $\mathbb{S}^{1}$ nos autoespaços de $\Delta$ são duas a duas não equivalentes.
} 
aos autovalores $\lambda$. Sabemos que $V_{\lambda}$ têm dimensões finitas, para todo $\lambda$ e são invariantes pela ação de $G$. Considere ainda $H_{0}$ a componente conexa de $H$ que contém a identidade $e_{G}$. Lembre que $H$ age em $T_{p} M$, onde p é o ponto base de $G / H$, via representação linear de isotropia

$$
\begin{gathered}
I s_{p}: H \longrightarrow G L\left(T_{p} M\right) \\
h \longmapsto(d h)_{p} ;
\end{gathered}
$$

se a ação de $H_{0}$ é transitiva sobre a esfera unitária de $T_{p} M$, então as representações

$$
\rho_{\lambda}: G \longrightarrow G L\left(V_{\lambda}\right)
$$

são irredutiveis, para todo $\lambda$.

Concretamente falando, este resultado se aplica aos espaços simétricos de posto 1, que consistem, além das esferas, dos seguintes espaços homogêneos com métricas $G$-invariantes:

(i.) o espaço projetivo real, com $M=\mathbb{R} P^{n}, G=O(n+1), H=O(n) \times\{-1,1\}, H_{0}=S O(n) \times$ $\{-1,1\}$

(ii.) o espaço projetivo complexo, com $M=\mathbb{C} P^{n}, G=U(n+1), H=H_{0}=U(n) \times U(1)$;

(iii.) o espaço projetivo quaterniônico, $\operatorname{com} M=\mathbb{H} P^{n}, G=S p(n+1), H=H_{0}=S p(n) \times S p(1)$;

(iv.) o plano de Cayley, com $M=\mathbb{P}^{2}(\mathrm{Ca}), G=F_{4}, H=H_{0}=\operatorname{Spin}(9)$.

Além disso, nestes casos, as dimensões dos autoespaços do laplaciano de $M$, com respeito a uma métrica $G$-invariante, formam uma sequência estritamente crescente, o que implica que os autoespaços são dois a dois não equivalentes. Portanto, a ação de $G$ em cada um dos espaços homogêneos listados acima, é harmonicamente livre.

Proposição 3.2.6. Seja $\left(M_{1}, g^{(1)}\right)$ uma variedade riemanniana compacta com curvatura escalar constante positiva e seja $\left(M_{2}, \bar{g}^{(2)}\right)$ uma variedade riemanniana compacta, com bordo $\partial M \neq \emptyset$, de curvatura escalar constante positiva e curvatura média do bordo nula. Assuma que o par $\left(g^{(1)}, \bar{g}^{(2)}\right)$ é não degenerado, no sentido da Definição 2.3.14. Considere a variedade produto $M=M^{(1)} \times M^{(2)}$, de dimensão $m=\operatorname{dim} M^{(1)}+\operatorname{dim} M^{(2)} \geq 3$ e bordo $\partial M=M^{(1)} \times \partial M^{(2)} \neq \emptyset$. Seja $\bar{g}_{s}=$ $g^{(1)} \oplus s \bar{g}^{(2)}$, com $s>0$, uma família de métricas, em M. Seja $G$ um grupo de Lie conexo agindo, harmonicamente livre, via isometrias em $M_{1}$ (ou $\left.M_{2}\right)$. Então, todo instante de degeneração para a familia de operadores $\left\{\mathcal{J}_{s}\right\}_{s>0}$ é um instante de bifurcação para a familia $\left\{\bar{g}_{s}\right\}_{s>0}$ de soluções do problema de Yamabe na variedade compacta $M$ cujo bordo é minimal.

Demonstração. Sem perda de generalidade, suponha que $G$ aja em $M_{1}$, então definimos a ação não trivial de $G$ sobre $M$ como

$$
\begin{aligned}
G \times M & \longrightarrow M \\
\left(\xi,\left(x_{1}, x_{2}\right)\right) & \longmapsto\left(\xi \cdot x_{1}, x_{2}\right),
\end{aligned}
$$

onde $x_{1} \in M_{1}$ e $x_{2} \in M_{2}$, isto é, a ação de $G$ sobre $M_{2}$ é trivial.

Lembre que associado à variedade $M_{1}$ existe um operador (simétrico) laplaciano $\Delta_{g^{(1)}}$, com autovalores

$$
0=\rho_{0}^{(1)}<\rho_{1}^{(1)}<\rho_{2}^{(1)}<\ldots
$$

de multiplicidades finitas $\mu_{i}^{(1)}$, com $i=0,1,2, \ldots$, a cada um dos quais está associado um autoespaço $V_{i}^{(1)}$, de dimensão finita igual a $\mu_{i}^{(1)}$, para cada $i \geq 0$. Como $G$ age por isometrias em $M_{1}$, podemos ver o autoespaço de $\rho_{i}^{(1)}$ como um $G$-espaço, de modo que $G$ tem uma antirrepresentação natural em $V_{i}^{(1)}$,

$$
\pi_{i}^{(1)}: G \longrightarrow G L\left(V_{i}^{(1)}\right)
$$


dada por $\pi_{i}^{(1)}(\xi) f=f \circ \xi, \forall f \in V_{i}^{(1)}$.

Para a variedade $M_{2}$,

$$
0=\rho_{0}^{(2)}<\rho_{1}^{(2)}<\rho_{2}^{(2)}<\ldots
$$

é a sequência de todos os autovalores distintos do operador $\Delta_{\bar{g}^{(2)}}$, sujeito à condição de Neumann no bordo,

$$
\left\{\begin{aligned}
\Delta_{\bar{g}^{(2)}} f^{(2)} & =\rho_{j}^{(2)} f^{(2)}, \text { on } M^{(2)}, \\
\partial_{\eta_{2}} f^{(2)} & =0, \text { on } \partial M^{(2)}
\end{aligned}\right.
$$

a cada um dos quais está associado um autoespaço $V_{j}^{(2)}$, de dimensão finita igual a $\mu_{j}^{(2)}$, que denota a multiplicidade geométrica de $\rho_{j}^{(2)}$, para cada $j \geq 0$. Como a ação de $G$ em $M_{2}$ é trivial, a representação de $G$ no autoespaço $V_{j}^{(2)}$ é a representação trivial, isto é,

$$
\pi_{j}^{(2)}: G \longrightarrow G L\left(V_{j}^{(2)}\right)
$$

é dada por $\pi_{j}^{(2)}(\xi) f=f \circ i d=f, \forall f \in V_{j}^{(2)}$.

Lembre ainda que, na variedade produto, $M$, o operador laplaciano é dado por

$$
\Delta_{\bar{g}_{s}}=\Delta_{g^{(1)}} \otimes I+\frac{1}{s}\left(I \otimes \Delta_{\bar{g}^{(2)}}\right)
$$

e tem autovalores $\rho_{i, j}=\rho_{i}^{(1)}+\frac{1}{s} \rho_{j}^{(2)}$, com multiplicidades $\mu_{i, j}=\mu_{i}^{(1)} \cdot \mu_{j}^{(2)}$, que são as dimensões dos respectivos autoespaços $V_{i, j}=V_{i}^{(1)} \otimes V_{j}^{(2)}$, para todo $i, j \geq 0$. Se $\Sigma\left(\Delta_{\bar{g}_{s}}\right)$ é o espectro do operador laplaciano na variedade produto $\left(M, \bar{g}_{s}\right)$, o espectro do operador $\mathcal{J}_{s}=\Delta_{\bar{g}_{s}}-\frac{R_{\bar{g}_{s}}}{m-1}$ é dado por

$$
\Sigma\left(\mathcal{J}_{s}\right)=\left\{\lambda-\frac{R_{\bar{g}_{s}}}{m-1}: \lambda \in \Sigma\left(\Delta_{\bar{g}_{s}}\right) \backslash\{0\}\right\} ;
$$

explicitamente,

$$
\Sigma\left(\mathcal{J}_{s}\right)=\left\{\sigma_{i, j}(s): i, j \geq 0, i+j>0\right\}
$$

onde os autovalores, são dados por

$$
\sigma_{i, j}(s)=\left(\rho_{i}^{(1)}-\frac{R_{g^{(1)}}}{m-1}\right)+\frac{1}{s}\left(\rho_{j}^{(2)}-\frac{R_{\bar{g}^{(2)}}}{m-1}\right),
$$

e satisfazem o problema de autovalor com condições de Neumann na variedade produto com bordo

$$
\left\{\begin{aligned}
\mathcal{J}_{s} f & =\sigma_{i, j} f, \text { on } M \\
\partial_{\eta_{s}} f & =0, \text { on } \partial M
\end{aligned}\right.
$$

onde a função $f=f^{(1)} \otimes f^{(2)}$ é autofunção de $\mathcal{J}_{s}$ associada ao autovalor $\sigma_{i, j}$, se e somente se é autofunção de $\Delta_{\bar{g}_{s}}$ associada ao autovalor $\rho_{i, j} \neq 0$, portanto $\sigma_{i, j}$ têm multiplicidade geométrica igual ao produto $\mu_{i}^{(1)} \cdot \mu_{j}^{(2)}$.

Agora, para cada autovalor $\rho_{i, j} \neq 0$ de $\Delta_{\bar{g}_{s}}$ (ou, equivalentemente, para cada autovalor $\sigma_{i, j}$ de $\mathcal{J}_{s}$ ), existe uma representação de $G$ no autoespaço $V_{i, j}=V_{i}^{(1)} \otimes V_{j}^{(2)}$ dada por

$$
\begin{aligned}
\pi_{i, j}: G & \longrightarrow G L\left(V_{i, j}\right) \\
\xi & \longmapsto \pi_{i, j}(\xi)
\end{aligned}
$$


onde

$$
\pi_{i, j}(\xi) f=\pi_{i}^{(1)}(\xi) \otimes \pi_{j}^{(2)}(\xi) f_{1} \otimes f_{2}=\pi_{i}^{(1)}(\xi) f_{1} \otimes \pi_{j}^{(2)}(\xi) f_{2}=\left(f_{1} \circ \xi\right) \otimes\left(f_{2} \circ i d\right)=\left(f_{1} \circ \xi\right) \otimes f_{2},
$$

para todo $g \in G$ e $f \in V_{i, j}$.

Seja $s_{*}$ um instante de degeneração para a família de métricas $\left\{\bar{g}_{s}\right\}_{s}$, em particular, $s_{*}$ pode ser um instante neutro. Seja $\varepsilon>0$ suficientemente pequeno para que $s_{*}$ seja o único instante de degeneração de $\mathcal{J}_{(} \cdot$ ) no intervalo $\left[s_{*}-\varepsilon, s_{*}+\varepsilon\right]$, em particular, $\mathcal{J}_{(}$. ) é não singular nos extremos desse intervalo. As representações de $G$ nos autoespaços negativos de $\mathcal{J}_{s_{*}-\varepsilon}$ e $\mathcal{J}_{s_{*}+\varepsilon}$, denotadas por

$$
\pi_{s_{*}-\varepsilon}^{-}: G \longrightarrow G L\left(V_{s_{*}-\varepsilon}^{-}\right) \quad \text { e } \quad \pi_{s_{*}+\varepsilon}^{-}: G \longrightarrow G L\left(V_{s_{*}+\varepsilon}^{-}\right),
$$

respectivamente, são dadas pelas somas diretas das representações de $G$ nos autoespaços $V_{i, j}$ associados aos autovalores negativos, $\sigma_{i, j}(\cdot)$, do operador $\mathcal{J}_{(} \cdot$ ) nos instantes $s_{*}-\varepsilon$ e $s_{*}+\varepsilon$. Note que o número de autovalores negativos e as dimensões dos autoespaços associados a estes autovalores para os operadores $J_{s_{*}-\varepsilon}$ e $J_{s_{*}+\varepsilon}$ coincidem, exceto para aqueles autovalores que têm $s_{*}$ como zero. Denote por $V_{0}$ a soma direta dos autoespaços associados a autovalores que assumem valores negativos para todo $s \in\left[s_{*}-\varepsilon, s_{*}+\varepsilon\right]$.

Seja $\sigma_{i, j}$ um autovalor que se anula em $s_{*}$. Se $\sigma_{i, j}$ é crescente o autoespaço $V_{i, j}$ faz parte do autoespaço negativo do operador $\mathcal{J}_{s}$, para todo $s<s_{*}$, mas a medida que $s$ varia, passando por $s_{*}$, isto é, quando $s>s_{*}, V_{i, j}$ deixa de fazer parte do autoespaço negativo de $\mathcal{J}_{s}$. O contrário ocorre se $\sigma_{i, j}$ é decrescente, neste caso, $V_{i, j}$ passará a fazer parte do autoespaço negativo de $\mathcal{J}_{s}$, somente quando $s>s_{*}$. Portanto, podemos escrever o autoespaço negativo do operador $\mathcal{J}_{s_{*}-\varepsilon}$ como

$$
V_{s_{*}-\varepsilon}^{-}=V_{0} \oplus\left(\bigoplus_{k=1}^{r}\left(V_{i_{k}}^{(1)} \otimes V_{j_{k}}^{(2)}\right)\right),
$$

onde $V_{i_{k}, j_{k}}=V_{i_{k}}^{(1)} \otimes V_{j_{k}}^{(2)}$ é o autoespaço associado a autovalores crescentes, tais que $\sigma_{i_{k}, j_{k}}\left(s_{*}\right)=0$. Analogamente, o autoespaço negativo do operador $\mathcal{J}_{s_{*}+\varepsilon}$ pode ser escrito como

$$
V_{s_{*}+\varepsilon}^{-}=V_{0} \oplus\left(\bigoplus_{k=r+1}^{r+s}\left(V_{i_{k}}^{(1)} \otimes V_{j_{k}}^{(2)}\right)\right),
$$

onde $V_{i_{k}, j_{k}}=V_{i_{k}}^{(1)} \otimes V_{j_{k}}^{(2)}$ é o autoespaço associado a autovalores decrescentes, tais que $\sigma_{i_{k}, j_{k}}\left(s_{*}\right)=$ 0 .

Veja que $\left\{V_{i_{k}}^{(1)}\right\}_{k=1}^{r+s}$ e $\left\{V_{j_{k}}^{(2)}\right\}_{k=1}^{r+s}$ são famílias de autoespaços, dois a dois distintos, de $\Delta_{g^{(1)}} \mathrm{e}$ $\Delta_{\bar{g}^{(2)}}$, respectivamente. Os pares $\left(i_{k}, j_{k}\right)$ são todos diferentes, mais que isso,

$$
\left(i_{p}, j_{p}\right) \neq\left(i_{q}, j_{q}\right) \text { e } \sigma_{i_{p}, j_{p}}\left(s_{*}\right)=\sigma_{i_{q}, j_{q}}\left(s_{*}\right)=0 \Longrightarrow i_{p} \neq i_{q} \text { e } j_{p} \neq j_{q}
$$

de fato, se $i_{p}=i_{q}$, como a sequência $j \mapsto \rho_{j}^{(2)}$ é estritamente crescente, existe um único $j_{p}$ tal que $\sigma_{i_{p}, j_{p}}\left(s_{*}\right)=0, \operatorname{logo} j_{p}=j_{q}$. Simetricamente, se $j_{p}=j_{q}$, então $i_{p}=i_{q}$.

A representação de $G$ no autoespaço negativo $V_{s_{*}-\varepsilon}^{-}$é dada por

$$
\pi_{s_{*}-\varepsilon}^{-}=\pi_{0} \oplus\left(\bigoplus_{k=1}^{r}\left(\pi_{i_{k}}^{(1)} \otimes \pi_{j_{k}}^{(2)}\right)\right),
$$

e a representação de $G$ no autoespaço negativo $V_{s_{*}+\varepsilon}^{-}$é dada por

$$
\pi_{s_{*}+\varepsilon}^{-}=\pi_{0} \oplus\left(\bigoplus_{k=r+1}^{r+s}\left(\pi_{i_{k}}^{(1)} \otimes \pi_{j_{k}}^{(2)}\right)\right)
$$


BIFURCAÇÃO EQUIVARIANTE DE SOLUÇÕES DO PROBLEMA DE YAMABE EM VARIEDADES

onde $\pi_{j_{k}}^{(2)}$ é a representação trivial. Sabemos que, se $U$ e $W$ são espaços vetoriais de dimensões finitas, $U \cdot \operatorname{dim} W=\underbrace{U \times U \times \ldots \times U}_{\operatorname{dim} W \text { vezes }}$, tem dimensão $\operatorname{dim} U \cdot \operatorname{dim} W$ e é isomorfo a $U \otimes W, \operatorname{logo}$,

$$
\bigoplus_{k=1}^{r} V_{i_{k}}^{(1)} \cdot \operatorname{dim} V_{j_{k}}^{(2)} \simeq \bigoplus_{k=1}^{r} V_{i_{k}}^{(1)} \otimes V_{j_{k}}^{(2)} \quad \text { e } \quad \bigoplus_{k=r+1}^{r+s} V_{i_{k}}^{(1)} \otimes V_{j_{k}}^{(2)} \simeq \bigoplus_{k=r+1}^{r+s} V_{i_{k}}^{(1)} \cdot \operatorname{dim} V_{j_{k}}^{(2)}
$$

como a ação de $G$ em $M_{1}$ é harmonicamente livre, não existe isomorfismo entre os espaços

$$
\bigoplus_{k=1}^{r} V_{i_{k}}^{(1)} \otimes V_{j_{k}}^{(2)} \text { e } \bigoplus_{k=r+1}^{r+s} V_{i_{k}}^{(1)} \otimes V_{j_{k}}^{(2)}
$$

Portanto, $\left(\pi_{s_{*}-\varepsilon}^{-}, V_{s_{*}-\varepsilon}^{-}\right)$e $\left(\pi_{s_{*}+\varepsilon}^{-}, V_{s_{*}+\varepsilon}^{-}\right)$são não-equivalentes e o resultado segue do Teorema 3.1.2. 


\section{Parte III}

Apêndice 



\section{Apêndice A}

\section{O Funcional de Hilbert-Einstein}

O funcional de Hilbert-Einstein tem esse nome em referência ao matemático David Hilbert e ao físico Albert Einstein por ter aparecido em seus respectivos artigos [24], [16], publicados quase que simultaneamente, sobre a teoria de gravitação e relatividade. Apresentaremos abaixo todos os cálculos para obtenção da primeira e segunda variações do funcional de Hilbert-Einstein, bem como considerações a respeito de seus pontos críticos.

\section{A.1 Primeira Variação do Funcional de Hilbert-Einstein}

Seja $(M, \bar{g})$ uma variedade riemanniana compacta orientada de dimensão $m \geq 3$, com bordo $\partial M \neq \emptyset$. O funcional de Hilbert-Einstein fica assim definido

$$
\begin{aligned}
F: \mathcal{M}^{k, \alpha}(M) & \longrightarrow \mathbb{R} \\
\bar{g} & \longmapsto \int_{M} R_{\bar{g}} \omega_{\bar{g}} .
\end{aligned}
$$

onde $\mathcal{M}^{k, \alpha}(M)$ é a variedade de dimensão infinita de métricas riemannianas de classe $\mathcal{C}^{k, \alpha}$ sobre $M$, com $k \geq 3$ e $0<\alpha \leq 1$. A curvatura escalar de $M$ com respeito à métrica $\bar{g}$ é denotada por $R_{\bar{g}}$ e $\omega_{\bar{g}}$ é a forma volume definida em $M$ com respeito à métrica $\bar{g}$. O funcional $F$ é suave sobre $\mathcal{M}^{k, \alpha}(M)$ e sobre as $\mathcal{C}^{k, \alpha}$-classes conformes $[\bar{g}]$.

Como $\mathcal{M}^{k, \alpha}(M)$ é um cone aberto de $\Gamma^{k, \alpha}\left(T^{*} M \otimes T^{*} M\right)$, sabemos que

$$
T_{\bar{g}} \mathcal{M}^{k, \alpha}(M)=\Gamma^{k, \alpha}\left(T^{*} M \otimes T^{*} M\right) .
$$

Assim, seja $\bar{g}(t)=g+t h$ uma variação da métrica $\bar{g} \in \mathcal{M}^{k, \alpha}(M)$ na direção do (0,2)-tensor simétrico $h$ sobre $M$, isto é, $\bar{g}(0)=\bar{g}$ e $\left.\frac{d}{d t} \bar{g}(t)\right|_{t=0}=h$. Então, podemos calcular a primeira variação do funcional $F$, na direção $h$,

$$
\begin{aligned}
\delta F(\bar{g}) h & =\delta \int_{M} R_{\bar{g}(t)} \omega_{\bar{g}(t)} \\
& =\left.\int_{M} \delta R_{\bar{g}(t)} \cdot \omega_{\bar{g}(t)}\right|_{t=0}+\left.R_{\bar{g}(t)}\right|_{t=0} \cdot \delta \omega_{\bar{g}(t)} \\
& =\int_{M} \delta R_{\bar{g}(t)} \cdot \omega_{\bar{g}}+R_{\bar{g}} \cdot \delta \omega_{\bar{g}(t)}
\end{aligned}
$$

onde $\delta:=\left.\frac{d}{d t}\right|_{t=0}$. Para obtermos a expressão final, precisamos calcular a derivada da curvatura escalar e da forma volume. 


\section{Primeira Variação da Curvatura Escalar}

Sabemos que a curvatura escalar é o traço do tensor de Ricci. Em coordenadas locais $x^{1}, \ldots, x^{n}$, numa vizinhança de um ponto $p \in M$, temos $R_{\bar{g}(t)}=\bar{g}(t)^{i j} R_{i j}(t)$, onde $\bar{g}(t)^{i j}$ são as coordenadas do inverso da métrica $\bar{g}(t)$ e $R_{i j}(t)$ são as coordenadas do tensor de Ricci com respeito à métrica $\bar{g}(t)$, daí

$$
\delta R_{\bar{g}(t)}=\delta \bar{g}(t)^{i j} \cdot R_{i j}(t)+\bar{g}(t)^{i j} \cdot \delta R_{i j}(t)
$$

Como $\left.\frac{d}{d t} \bar{g}(t)_{i j}\right|_{t=0}=h_{i j}$ e $\bar{g}(t)_{k i} \cdot \bar{g}(t)^{i j}=\delta_{k}^{j}$, onde $\delta_{k}^{j}$ é o delta de Kronecker (não confundir com o $\delta$ que indica a derivação em $t$ avaliada no $t=0$ ), temos

$$
0=\delta \bar{g}(t)_{k i} \cdot \bar{g}(t)^{i j}=h_{k i} \cdot \bar{g}(t)^{i j}+\bar{g}(t)_{k i} \cdot \delta \bar{g}(t)^{i j},
$$

portanto

$$
\delta \bar{g}(t)^{i j}=-\bar{g}(t)^{i k} h_{k i} \bar{g}(t)^{i j}=-\bar{g}(t)^{i k} h_{k}^{j}=-h^{i j},
$$

isto é, a primeira variação do inverso do tensor métrico $\bar{g}$ na direção $h$ é dada por

$$
\delta \bar{g}(t)^{i j}=-h^{i j} .
$$

O sistema de coordenadas locais $\left\{x^{i}\right\}_{i=1}^{m}$ em torno de $p \in M$, define um referencial local

$$
e_{i}=\frac{\partial}{\partial x^{i}}=\partial_{i},
$$

com $i=1, \ldots, m$, no espaço tangente à $M$ em $p$. Os símbolos de Chistoffel, $\Gamma_{i j}^{k}$, são definidos como os únicos coeficientes (funções suaves em $M$ ) tais que

$$
\nabla_{i} e_{j}=\Gamma_{i j}^{k} e_{k}
$$

onde $\nabla_{i}$ é a conexão riemanniana com respeito à métrica $g$, na direção $e_{i}$ (i.e. $\nabla_{i}=\nabla_{e_{i}}$ ) e por esta razão são chamados coeficientes da conexão riemanniana (em um referencial local). Como a conexão Levi-Civita é livre de torção, segue que $\Gamma_{i j}^{k}=\Gamma_{j i}^{k}$.

Lembremos que

Proposição A.1.1. Em coordenadas locais o tensor de Ricci pode ser escrito como

$$
R_{i j}=\nabla_{l} \Gamma_{i j}^{l}-\nabla_{i} \Gamma_{j l}^{l}+\left(\Gamma_{i j}^{u} \Gamma_{l u}^{l}-\Gamma_{l j}^{u} \Gamma_{i u}^{l}\right) .
$$

Demonstração. Sabemos que o tensor de Ricci é o traço do tensor de Riemann

$$
R_{i j}=R_{i l j}^{l}=g^{r s} R_{i r j s},
$$

onde

$$
R_{i l j}^{l}=\bar{g}\left(R\left(e_{i}, e_{l}\right) e_{j}, e_{l}^{*}\right)=d x^{l}\left(R\left(\partial_{i}, \partial_{l}\right) \partial_{j}\right)
$$

com $e_{l}^{*}=d x^{l}, e_{l}=\partial_{l}$ (vetores cotangentes da base dual, i.e. base de $T_{p}^{*} M$ ), são as coordenadas do (1,3)-tensor de Riemann

$$
R\left(e_{i}, e_{l}\right) e_{j}=\nabla_{\left[e_{i}, e_{l}\right]} e_{j}-\nabla_{e_{i}} \nabla_{e_{l}} e_{j}+\nabla_{e_{l}} \nabla_{e_{i}} e_{j},
$$

onde

$$
\left[e_{i}, e_{l}\right]=\nabla_{e_{i}} e_{l}-\nabla_{e_{l}} e_{i}
$$

De (A.4) e (A.6), vem

$$
\begin{aligned}
& {\left[e_{i}, e_{l}\right]=\Gamma_{i l}^{u} e_{u}-\Gamma_{l i}^{u} e_{u},} \\
& {\left[e_{i}, e_{l}\right]=\left(\Gamma_{i l}^{u}-\Gamma_{l i}^{u}\right) e_{u} .}
\end{aligned}
$$


Usando (A.4) e (A.7) podemos escrever (A.5) como

$$
\begin{aligned}
R\left(e_{i}, e_{l}\right) e_{j} & =\left(\Gamma_{i l}^{u} \nabla_{u} e_{j}-\Gamma_{l i}^{u} \nabla_{u} e_{j}\right)-\nabla_{i}\left(\Gamma_{l j}^{m} e_{m}\right)+\nabla_{l}\left(\Gamma_{i j}^{m} e_{m}\right) \\
& =\Gamma_{i l}^{u} \Gamma_{u j}^{m} e_{m}-\Gamma_{l i}^{u} \Gamma_{u j}^{m} e_{m}-\left(e_{i} \cdot \Gamma_{l j}^{m}\right) e_{m}-\Gamma_{l j}^{m} \nabla_{i} e_{m}+\left(e_{l} \cdot \Gamma_{i j}^{m}\right) e_{m}+\Gamma_{i j}^{m} \nabla_{l} e_{m} \\
& =\left(\Gamma_{i l}^{u} \Gamma_{u j}^{m}-\Gamma_{l i}^{u} \Gamma_{u j}^{m}-\left(e_{i} \cdot \Gamma_{l j}^{m}\right)+\left(e_{l} \cdot \Gamma_{i j}^{m}\right)-\Gamma_{l j}^{u} \Gamma_{i u}^{m}+\Gamma_{i j}^{u} \Gamma_{l u}^{m}\right) e_{m}
\end{aligned}
$$

(observe que, nos dois últimos somatórios, trocamos os índices $u$ por $m$, por exemplo, $\Gamma_{l j}^{m} \Gamma_{i m}^{u}$ ficou $\Gamma_{l j}^{u} \Gamma_{i u}^{m}$ ). Agora, pela simetria da métrica Levi-Civita (na verdade, já sabemos que (A.6) e (A.7) são nulas), temos

$$
R\left(e_{i}, e_{l}\right) e_{j}=\left(\left(e_{l} \cdot \Gamma_{i j}^{m}\right)-\left(e_{i} \cdot \Gamma_{l j}^{m}\right)-\Gamma_{l j}^{u} \Gamma_{i u}^{m}+\Gamma_{i j}^{u} \Gamma_{l u}^{m}\right) e_{m},
$$

donde

$\left(\left(e_{l} \cdot \Gamma_{i j}^{m}\right)-\left(e_{i} \cdot \Gamma_{l j}^{m}\right)-\Gamma_{l j}^{u} \Gamma_{i u}^{m}+\Gamma_{i j}^{u} \Gamma_{l u}^{m}\right) g\left(e_{m}, e_{k}^{*}\right)=\left(e_{l} \cdot \Gamma_{i j}^{k}\right)-\left(e_{i} \cdot \Gamma_{l j}^{k}\right)-\Gamma_{l j}^{u} \Gamma_{i u}^{k}+\Gamma_{i j}^{u} \Gamma_{l u}^{k}=R_{i l j}^{k}$.

Agora, $R_{i j}=R_{i l j}^{l}$, logo

$$
\begin{gathered}
R_{i j}=\left(e_{l} \cdot \Gamma_{i j}^{l}\right)-\left(e_{i} \cdot \Gamma_{l j}^{l}\right)-\Gamma_{l j}^{u} \Gamma_{i u}^{l}+\Gamma_{i j}^{u} \Gamma_{l u}^{l}, \\
R_{i j}=\nabla_{l} \Gamma_{i j}^{l}-\nabla_{i} \Gamma_{j l}^{l}+\left(\Gamma_{i j}^{u} \Gamma_{l u}^{l}-\Gamma_{l j}^{u} \Gamma_{i u}^{l}\right) .
\end{gathered}
$$

Assim, para calcularmos a primeira variação do tensor de Ricci, precisaremos da primeira variação dos símbolos de Christoffel. Da expressão (A.4) temos

$$
\bar{g}\left(\nabla_{e_{i}} e_{j}, e_{l}\right)=\bar{g}\left(\Gamma_{i j}^{k} e_{k}, e_{l}\right)=\Gamma_{i j}^{k} \bar{g}\left(e_{k}, e_{l}\right)=\Gamma_{i j}^{k} \bar{g}_{k l} .
$$

Pela fórmula de Koszul,

$$
\begin{aligned}
2 \bar{g}\left(\nabla_{e_{i}} e_{j}, e_{l}\right) & =e_{j} \cdot \bar{g}\left(e_{i}, e_{l}\right)-e_{l} \cdot \bar{g}\left(e_{j}, e_{i}\right)+e_{i} \cdot \bar{g}\left(e_{l}, e_{j}\right)-\bar{g}\left(\left[e_{j}, e_{i}\right], e_{l}\right)-\bar{g}\left(\left[e_{j}, e_{l}\right], e_{i}\right)-\bar{g}\left(\left[e_{i}, e_{l}\right], e_{j}\right) \\
& =\frac{\partial}{\partial x_{j}} \bar{g}_{i l}-\frac{\partial}{\partial x_{l}} \bar{g}_{j i}+\frac{\partial}{\partial x_{i}} \bar{g}_{l j}
\end{aligned}
$$

donde

$$
\Gamma_{i j}^{k}=\frac{1}{2} \bar{g}^{k l}\left(\frac{\partial}{\partial x_{i}} \bar{g}_{j l}+\frac{\partial}{\partial x_{j}} \bar{g}_{i l}-\frac{\partial}{\partial x_{l}} \bar{g}_{i j}\right) .
$$

Então, a primeira variação de $\Gamma_{i j}^{k}$ na direção $h$ é dada por

$$
\delta \Gamma_{i j}^{k}(t)=\frac{1}{2} \bar{g}^{k l}\left(\frac{\partial}{\partial x_{i}} h_{j l}+\frac{\partial}{\partial x_{j}} h_{i l}-\frac{\partial}{\partial x_{l}} h_{i j}\right)-\frac{1}{2} h^{k l}\left(\frac{\partial}{\partial x_{i}} \bar{g}_{j l}+\frac{\partial}{\partial x_{j}} \bar{g}_{i l}-\frac{\partial}{\partial x_{l}} \bar{g}_{i j}\right)
$$

Observe que, em um referencial geodésico em $p \in M$, os símbolos de Christoffel se anulam em $p: \Gamma_{i j}^{k}(p)=0$. Consequentemente, $\partial_{i} \tau_{j k}=\nabla_{i} \tau_{j k}$ em $p$ para qualquer tensor $\tau$, em particular $\partial_{i} g_{j k}(p)=0$. Portanto, em $p$, obtemos

$$
\delta \Gamma_{i j}^{k}(p)=\frac{1}{2} \bar{g}^{k l}(p)\left(\frac{\partial}{\partial x_{i}} h_{j l}+\frac{\partial}{\partial x_{j}} h_{i l}-\frac{\partial}{\partial x_{l}} h_{i j}\right),
$$

mas ambos os lados da igualdade acima são componentes de tensores, portanto esse resultado vale em qualquer ponto de qualquer sistema de coordenadas. 
Agora podemos calcular a primeira variação do tensor de Ricci,

$$
\delta R_{i j}(t)=\delta \nabla_{l} \Gamma_{i j}^{l}(t)-\delta \nabla_{i} \Gamma_{j l}^{l}(t)+\delta\left(\Gamma_{i j}^{u} \Gamma_{l u}^{l}-\Gamma_{l j}^{u} \Gamma_{i u}^{l}\right)(t) .
$$

Considerando coordenadas normais, a regra do produto e o fato de $\Gamma_{i j}^{k}(p)=0$ em $p$, resultam em

$$
\delta\left(\Gamma_{i j}^{u} \Gamma_{l u}^{l}-\Gamma_{l j}^{u} \Gamma_{i u}^{l}\right)(p)=0,
$$

$\log 0$

$$
\begin{aligned}
\delta R_{i j}(t) & =\nabla_{l} \delta \Gamma_{i j}^{l}(t)-\nabla_{i} \delta \Gamma_{j l}^{l}(t) \\
& =\frac{1}{2} \nabla_{l}\left[\left(\frac{\partial}{\partial x_{i}} h_{j m}+\frac{\partial}{\partial x_{j}} h_{i m}-\frac{\partial}{\partial x_{m}} h_{i j}\right) \bar{g}^{l m}(t)\right] \\
& -\frac{1}{2} \nabla_{i}\left[\left(\frac{\partial}{\partial x_{j}} h_{l m}+\frac{\partial}{\partial x_{l}} h_{j m}-\frac{\partial}{\partial x_{m}} h_{j l}\right) \bar{g}^{l m}(t)\right]
\end{aligned}
$$

Note que

$$
\frac{\partial}{\partial x_{l}} h_{j m} \cdot \bar{g}^{l m}(t)-\frac{\partial}{\partial x_{m}} h_{j l} \cdot \bar{g}^{l m}(t)=\partial_{l} h_{j}^{l}-\partial_{m} h_{j}^{m}=\partial_{s} h_{j}^{s}-\partial_{s} h_{j}^{s}=0,
$$

além disso

$$
\nabla_{i} \bar{g}^{l m}(t)=\nabla_{i} \bar{g}-t \nabla_{i} h^{l m} \Rightarrow \nabla_{i} \bar{g}^{l m}(0)=0
$$

Portanto,

$$
\delta R_{i j}(t)=\frac{1}{2}\left[\left(\nabla_{l} \partial_{i} h_{j m}+\nabla_{l} \partial_{j} h_{i m}-\nabla_{l} \partial_{m} h_{i j}\right) \bar{g}^{l m}(t)-\nabla_{i} \partial_{j} h_{l m} \bar{g}^{l m}(t)\right]_{t=0}
$$

e a primeira variação do tensor de Ricci na direção $h$ é

$$
\delta R_{i j}(t)=\frac{1}{2}\left(\nabla_{l} \nabla_{i} h_{j m}+\nabla_{l} \nabla_{j} h_{i m}-\nabla_{l} \nabla_{m} h_{i j}-\nabla_{i} \nabla_{j} h_{l m}\right) \bar{g}^{l m}
$$

Agora, substituindo as expressões (A.3) e (A.9) em (A.1), temos

$$
\delta R_{\bar{g}(t)}=-h^{i j} R_{i j}+\frac{1}{2} \bar{g}^{i j}\left(\nabla_{l} \nabla_{i} h_{j m}+\nabla_{l} \nabla_{j} h_{i m}-\nabla_{l} \nabla_{m} h_{i j}-\nabla_{i} \nabla_{j} h_{l m}\right) \bar{g}^{l m} .
$$

Observe que

$$
\begin{aligned}
\frac{1}{2} \bar{g}^{i j}\left(\nabla_{l} \nabla_{i} h_{j m} \bar{g}^{l m}\right)+\frac{1}{2} \bar{g}^{i j}\left(\nabla_{l} \nabla_{j} h_{i m} \bar{g}^{l m}\right) & =\frac{1}{2} \bar{g}^{i j} \nabla_{l} \nabla_{i} h_{j}^{l}+\frac{1}{2} \bar{g}^{i j} \nabla_{l} \nabla_{j} h_{i}^{l} \\
& =\frac{1}{2} \nabla_{l} \nabla_{i} h^{i l}+\frac{1}{2} \nabla_{l} \nabla_{j} h^{j l} \\
& =\nabla_{i} \nabla_{j} h^{i j},
\end{aligned}
$$

analogamente, teremos

$$
\frac{1}{2} \bar{g}^{i j}\left(-\nabla_{l} \nabla_{m} h_{i j} \bar{g}^{l m}\right)+\frac{1}{2} \bar{g}^{i j}\left(-\nabla_{i} \nabla_{j} h_{l m} \bar{g}^{l m}\right)=-\nabla_{i} \nabla^{i}\left(h_{l m} g^{l m}\right)
$$

Finalmente, a primeira variação da curvatura escalar na direção $h$, é dada por

$$
\delta R_{\bar{g}}(h)=-h^{i j} R_{i j}+\nabla_{i}\left[\nabla_{j} h^{i j}-\nabla^{i}\left(g^{l m} h_{l m}\right)\right]
$$




\section{Primeira Variação da Forma Volume}

Em coordenadas locais $x^{1}, \ldots, x^{m}$, a forma volume associada à métrica $\bar{g}$ é representada por

$$
\omega_{\bar{g}}=\sqrt{\operatorname{det}[\bar{g}]} d x^{1} \wedge \ldots \wedge d x^{m},
$$

onde os colchetes indicam a matriz do tensor que aparece entre eles.

A primeira variação da forma volume é obtida a partir de

$$
\begin{aligned}
\delta \omega_{\bar{g}(t)} & =\delta\left(\sqrt{\operatorname{det}[\bar{g}(t)]} d x^{1} \wedge \ldots \wedge d x^{m}\right) \\
& =\frac{1}{2} \frac{1}{\sqrt{\operatorname{det}[\bar{g}]}}(\delta \operatorname{det}[\bar{g}(t)]) d x^{1} \wedge \ldots \wedge d x^{m} .
\end{aligned}
$$

De modo que basta calcularmos a derivada do determinante, que é conhecida, dada por

$$
\frac{d}{d t} \operatorname{det}[\bar{g}(t)]=\operatorname{tr}\left(\operatorname{ad}[\bar{g}(t)] \cdot \frac{d}{d t}[\bar{g}(t)]\right)=\operatorname{tr}\left(\operatorname{det}[\bar{g}(t)] \cdot[\bar{g}(t)]^{-1}[h]\right)=\operatorname{det}[\bar{g}(t)] \cdot \operatorname{tr}\left([\bar{g}(t)]^{-1}[h]\right),
$$

onde ad denota a adjunta da matriz em questão. Daí,

$$
\delta \operatorname{det}[\bar{g}(t)]=\operatorname{det}[\bar{g}] \cdot \operatorname{tr}\left([\bar{g}]^{-1}[h]\right)=\operatorname{det}[\bar{g}] \cdot \operatorname{tr}_{\bar{g}}[h] .
$$

Portanto, a primeira variação da forma volume na direção $h$ é

$$
\begin{aligned}
& \delta \omega_{\bar{g}(t)}=\frac{1}{2} \frac{1}{\sqrt{\operatorname{det}[\bar{g}]}} \operatorname{det}[\bar{g}] \cdot \operatorname{tr}_{\bar{g}}[h] d x^{1} \wedge \ldots \wedge d x^{m}=\frac{1}{2} \sqrt{\operatorname{det}[\bar{g}]} \operatorname{tr}_{\bar{g}}[h] d x^{1} \wedge \ldots \wedge d x^{m} \\
& \delta \omega_{\bar{g}(t)}=\frac{1}{2} \operatorname{tr}_{\bar{g}}[h] \omega_{\bar{g}} .
\end{aligned}
$$

Observação A.1.2. As expressões são todas equivalentes

$$
\operatorname{tr}_{\bar{g}}(h)=\operatorname{tr}_{\bar{g}} h=\operatorname{tr}\left(\bar{g}^{-1} h\right)=\left\langle\bar{g}^{-1}, h\right\rangle=g^{i j} h_{i j} .
$$

\section{Primeira Variação do Funcional de Hilbert-Einstein}

A primeira expressão que tínhamos para a variação do funcional H-E era

$$
\delta F(\bar{g}) h=\int_{M} \delta R_{\bar{g}(t)} \cdot \omega_{\bar{g}}+R_{\bar{g}} \cdot \delta \omega_{\bar{g}(t)} .
$$

Substituindo as expressões encontradas para a primeira variação da curvatura escalar (A.10) e para a primeira variação da forma volume (A.11), temos

$$
\begin{aligned}
\delta F(\bar{g}) h & =\int_{M}-h^{i j} R_{i j}+\nabla_{i}\left[\nabla_{j} h^{i j}-\nabla^{i}\left(g^{l m} h_{l m}\right)\right]+R_{\bar{g}} \frac{1}{2} g^{i j} h_{i j} \omega_{\bar{g}} \\
& =\int_{M} \frac{1}{2} g^{i j} h_{i j} R_{\bar{g}}-h^{i j} R_{i j} \omega_{\bar{g}}+\int_{M} \nabla_{i}\left[\nabla_{j} h^{i j}-\nabla^{i}\left(g^{l m} h_{l m}\right)\right] \omega_{\bar{g}},
\end{aligned}
$$

pelo Teorema da Divergência, vem

$$
\delta F(\bar{g}) h=\int_{M}-h^{i j}\left(R_{i j}-\frac{1}{2} \bar{g}_{i j} R_{\bar{g}}\right) \omega_{\bar{g}}-\int_{\partial M}\left[\nabla_{j} h^{i j}-\nabla^{i}\left(g^{l m} h_{l m}\right)\right] \eta_{i} \sigma_{\bar{g}}
$$

onde $\eta_{i}$ são coordenadas do campo unitário normal ao bordo (apontando para dentro de $M$ ) com respeito à métrica $\bar{g}$ e $\sigma_{\bar{g}}$ é o elemento de área induzido pela forma volume no bordo de $M$. Podemos 
escrever a expressão anterior como

$$
\delta F(\bar{g}) h=-\int_{M}\left\langle R i c_{\bar{g}}-\frac{1}{2} R_{\bar{g}} \bar{g}, h\right\rangle_{\bar{g}} \omega_{\bar{g}}-\int_{\partial M}\left\langle\nabla_{\bar{g}} h-\mathrm{d} \operatorname{tr}_{\bar{g}} h, \eta\right\rangle \sigma_{\bar{g}} .
$$

Agora, tome $p \in \partial M$ e seja $\left(x^{1}, \ldots, x^{m-1}\right)$ um sistema de coordenadas normais centrado em $p$ em uma vizinhança $U$ de $p,\left(x^{1}(p), \ldots, x^{m-1}(p)\right)=0$. Existe $V \subset U$ tal que, enquanto a geodésica partindo de $p$ na direção ortogonal ao bordo de $M$ estiver em $V$, poderá ser representada em coordenadas por $\left(0, \ldots, 0, x^{m}\right)$, então $\left(x^{1}, \ldots, x^{m}\right)$ é um sistema de coordenadas Fermi em $p \in \partial M$. Como já vimos, neste sistema de coordenadas, a métrica $\bar{g}$ é a métrica euclidiana

$$
\bar{g}=\bar{g}_{\alpha \beta} d x^{\alpha} \otimes d x^{\beta}+d x^{m} \otimes d x^{m},
$$

$\operatorname{com} \alpha, \beta=1, \ldots, m-1$, e vale

$$
\begin{aligned}
& \bar{g}_{m \beta}=0, \text { para } \beta=1, \ldots, m-1 \\
& \bar{g}_{m m}=1
\end{aligned}
$$

Portanto, no ponto $p \in \partial M$, temos

$$
\left\langle\nabla_{\bar{g}} h-\mathrm{d} \operatorname{tr}_{\bar{g}} h, \eta\right\rangle=\left[\nabla_{j} h^{i j}-\nabla^{i}\left(g^{l m} h_{l m}\right)\right] \eta_{i}=\nabla_{j} h_{m}^{j}-\nabla_{m} h_{k}^{k}=\nabla_{\alpha} h_{m}^{\alpha}-\nabla_{m} h_{\alpha}^{\alpha},
$$

$$
\left\langle\nabla_{\bar{g}} h-\mathrm{d} \operatorname{tr}_{\bar{g}} h, \eta\right\rangle=\nabla_{\alpha} h_{m}^{\alpha}-\nabla_{m} h_{\alpha}^{\alpha}=\bar{g}^{\alpha \beta} \nabla_{\alpha} h_{\beta m}-\nabla_{m} \operatorname{tr}_{\bar{g}} h
$$

Por outro lado,

$$
\begin{aligned}
\delta H_{\bar{g}(t)} & =\delta\left(\bar{g}^{\alpha \beta}(t) I I_{\alpha \beta}(t)\right) \\
& =\delta \bar{g}^{\alpha \beta}(t) I I_{\alpha \beta}+\bar{g}^{\alpha \beta} \delta I I_{\alpha \beta}(t),
\end{aligned}
$$

onde $I I_{\alpha \beta}(t)=\bar{g}(t)\left(\nabla_{\alpha} \partial_{\beta}, \eta_{\bar{g}(t)}\right)$ é a segunda forma fundamental.

A variação da segunda forma fundamental fica

$$
\begin{aligned}
\delta I_{\alpha \beta}(t) & =(\delta \bar{g}(t))\left(\nabla_{\alpha} \partial_{\beta}, \eta_{\bar{g}}\right)+\bar{g}\left(\delta \nabla_{\alpha} \partial_{\beta}, \eta_{\bar{g}}\right)+\bar{g}\left(\nabla_{\alpha} \partial_{\beta}, \delta \eta_{\bar{g}(t)}\right) \\
& =h\left(\Gamma_{\alpha \beta}^{k} \partial_{k}, \eta^{m}\right)+g\left(\delta \Gamma_{\alpha \beta}^{k} \partial_{k}, \eta^{m}\right)+g\left(\Gamma_{\alpha \beta}^{k} \partial_{k}, \delta \eta_{\bar{g}(t)}\right) \\
& =\Gamma_{\alpha \beta}^{k} h_{k m}+\delta \Gamma_{\alpha \beta}^{k} \bar{g}_{k m}+\Gamma_{\alpha \beta}^{k} g\left(\partial_{k}, \delta \eta_{\bar{g}(t)}\right) \\
& =\Gamma_{\alpha \beta}^{m} h_{m m}+\delta \Gamma_{\alpha \beta}^{m}+\Gamma_{\alpha \beta}^{m} \delta \eta_{\bar{g}(t)}^{m},
\end{aligned}
$$

onde

$$
\delta \Gamma_{\alpha \beta}^{m}=\frac{1}{2}\left(\frac{\partial}{\partial x_{\alpha}} h_{\beta m}+\frac{\partial}{\partial x_{\beta}} h_{\alpha m}-\frac{\partial}{\partial x_{m}} h_{\alpha \beta}\right)
$$

no ponto $p$ (vide A.8). Para calcularmos $\delta \eta_{\bar{g}(t)}^{m}$ em $p$, observe que $\eta_{\bar{g}(t)}^{m}=\sqrt{\bar{g}^{m m}(t)}$, daí

$$
\begin{aligned}
\delta \eta_{\bar{g}(t)}^{m} & =\delta \sqrt{\bar{g}^{m m}(t)} \\
& =\frac{1}{2} \frac{1}{{\sqrt{g^{m m}}}^{m m}} \delta \bar{g}^{m m}(t) \\
& =\frac{1}{2}\left(-h^{m m}\right) \\
& =-\frac{1}{2} \bar{g}^{l m} \bar{g}^{m k} h_{k l} \\
& =-\frac{1}{2} h_{m m}
\end{aligned}
$$


Portanto,

$$
\begin{aligned}
\delta I I_{\alpha \beta}(t) & =\Gamma_{\alpha \beta}^{m} h_{m m}+\frac{1}{2}\left(\frac{\partial}{\partial x_{\alpha}} h_{\beta m}+\frac{\partial}{\partial x_{\beta}} h_{\alpha m}-\frac{\partial}{\partial x_{m}} h_{\alpha \beta}\right)-\frac{1}{2} \Gamma_{\alpha \beta}^{m} h_{m m} \\
& =\frac{1}{2} \Gamma_{\alpha \beta}^{m} h_{m m}+\frac{1}{2}\left(\frac{\partial}{\partial x_{\alpha}} h_{\beta m}+\frac{\partial}{\partial x_{\beta}} h_{\alpha m}-\frac{\partial}{\partial x_{m}} h_{\alpha \beta}\right) .
\end{aligned}
$$

Note que, no ponto $p$,

$$
\begin{aligned}
H_{\bar{g}} & =\bar{g}^{\alpha \beta} I I_{\alpha \beta}(t) \\
& =\bar{g}^{\alpha \beta} \bar{g}\left(\nabla_{\alpha} \partial_{\beta}, \eta_{\bar{g}}\right) \\
& =\bar{g}^{\alpha \beta} \Gamma_{\alpha \beta}^{m} .
\end{aligned}
$$

Então, temos

$$
\begin{aligned}
\delta \bar{g}^{\alpha \beta}(t) I I_{\alpha \beta} & =-h^{\alpha \beta} I I_{\alpha \beta} \\
& =-\bar{g}^{l \alpha} g^{\beta k} h_{k l} I I_{\alpha \beta} \\
& =-\bar{g}\left(I I_{\bar{g}}, h\right) \\
\mathrm{e} & \\
\bar{g}^{\alpha \beta} \delta I I_{\alpha \beta}(t) & =\frac{1}{2} \bar{g}^{\alpha \beta} \Gamma_{\alpha \beta}^{m} h_{m m}+\frac{1}{2} \bar{g}^{\alpha \beta}\left(\nabla_{\alpha} h_{\beta m}+\nabla_{\beta} h_{\alpha m}-\nabla_{m} h_{\alpha \beta}\right) \\
& =\frac{1}{2} H_{\bar{g}} h_{m m}+\frac{1}{2} \bar{g}^{\alpha \beta} \nabla_{\alpha} h_{\beta m}+\frac{1}{2} \bar{g}^{\alpha \beta} \nabla_{\beta} h_{\alpha m}-\frac{1}{2} \nabla_{m} h_{\alpha}^{\alpha} \\
& =\frac{1}{2} H_{\bar{g}} h_{m m}+\bar{g}^{\alpha \beta} \nabla_{\alpha} h_{\beta m}-\frac{1}{2} \nabla_{m} \operatorname{tr}_{\bar{g}} h
\end{aligned}
$$

Daí, a equação (A.13) fica

$$
\delta H_{\bar{g}(t)}=-\bar{g}\left(I I_{\bar{g}}, h\right)+\frac{1}{2} H_{\bar{g}} h_{m m}+\bar{g}^{\alpha \beta} \nabla_{\alpha} h_{\beta m}-\frac{1}{2} \nabla_{m} \operatorname{tr}_{\bar{g}} h
$$

Agora, usando a expressão encontrada para $\delta H_{\bar{g}(t)}$ e (A.12), temos

$$
\left\langle\nabla_{\bar{g}} h-\mathrm{d} \operatorname{tr}_{\bar{g}} h, \eta\right\rangle=2 \delta H_{\bar{g}}+2\left\langle I I_{\bar{g}}, h\right\rangle-h_{m m} H_{\bar{g}}-g^{\alpha \beta} \nabla_{\alpha} h_{m \beta}
$$

Vejamos que, em $p$, temos

$$
\bar{g}^{\alpha \beta} \nabla_{\alpha} h_{m \beta}=\bar{g}^{\alpha \beta}\left(D_{\alpha} h_{m \beta}-\Gamma_{\alpha \beta}^{m} h_{m m}+\Gamma_{\alpha m}^{r} h_{\beta r}\right),
$$

onde $r=1, \ldots, m-1$ e $D$ denota a conexão induzida no bordo, daí

$$
\begin{aligned}
\bar{g}^{\alpha \beta} \nabla_{\alpha} h_{m \beta} & =\nabla_{\alpha} h_{m}^{\alpha}-H_{\bar{g}} h_{m m}+\bar{g}^{\alpha \beta} \Gamma_{\alpha m}^{r} h_{\beta r} \\
& =\nabla_{\alpha} h_{m}^{\alpha}-H_{\bar{g}} h_{m m}+\bar{g}^{\alpha r} \bar{g}^{s \beta} I I_{\alpha \beta} h_{r s} \\
& =\nabla_{\alpha} h_{m}^{\alpha}-H_{\bar{g}} h_{m m}+\left\langle I I_{\bar{g}}, h\right\rangle .
\end{aligned}
$$

Diante disso, a expressão (A.14) fica

$$
\left\langle\nabla_{\bar{g}} h-\mathrm{d} \operatorname{tr}_{\bar{g}} h, \eta\right\rangle=2 \delta H_{\bar{g}}+\left\langle I I_{\bar{g}}, h\right\rangle-\nabla_{\alpha} h_{m}^{\alpha},
$$


como $\int_{\partial M} \nabla_{\alpha} h_{m}^{\alpha} \sigma_{\bar{g}}=0$, obtemos, finalmente, a expressão desejada

$$
\delta F(\bar{g}) h=-\int_{M}\left\langle R i c_{\bar{g}}-\frac{R_{\bar{g}}}{2} \bar{g}, h\right\rangle_{\bar{g}} \omega_{\bar{g}}-2 \int_{\partial M}\left(\delta H_{\bar{g}}+\frac{1}{2}\left\langle I I_{\bar{g}}, h\right\rangle\right) \sigma_{\bar{g}}
$$

Observação A.1.3. É interessante mencionar que...

- ...se $\partial M=\emptyset$ ou se supormos que a variedade $M$ tem bordo minimal, então a segunda parcela da expressão obtida é nula.

- ...o termo

$$
G=R i c_{\bar{g}}-\frac{R_{\bar{g}}}{2} \bar{g}
$$

é o tensor de Einstein, que aparece nas equações de campo de Einstein para Gravitação e mede a curvatura do espaço-tempo de forma consistente com as leis de conservação de energia.

\section{Pontos Críticos}

Dada uma variedade riemanniana $(M, g)$, compacta, sem bordo, de dimensão $m \geq 3$, os pontos críticos do funcional $F$, para $g \in \mathcal{M}^{k}(M)$ e $h \in \Gamma^{k}\left(T^{*} M \otimes T^{*} M\right)$, são as métricas riemannianas de Ricci "flat". Basta observar que

$$
\delta F(g) h=-\int_{M}\left\langle R i c_{g}-\frac{1}{2} R_{g} g, h\right\rangle_{g} \omega_{g}=0, \forall h \in \Gamma^{k}\left(T^{*} M \otimes T^{*} M\right),
$$

implica em Ric $_{g}=\frac{1}{2} R_{g} g$. Tomando o traço, temos $R_{g}=\frac{m}{2} R_{g}$ e como $m \geq 3$, segue que $R_{g}=0$, donde $\operatorname{Ric}_{g}=0$.

Restringindo $F$ às métricas de classe $\mathcal{C}^{k}$ em $M$, de volume 1, variedade denotada por $\mathcal{M}^{k}(M)_{1}$, usamos o método dos multiplicadores de Lagrange para calcular os pontos críticos: $g \in \mathcal{M}^{k}(M)$ é um ponto crítico do funcional $F$ sujeito ao vínculo $\mathcal{V}(g)-1=0$, se e somente se existe $\lambda \in \mathbb{R} \backslash\{0\}$ tal que

$$
\delta F_{\lambda}(g)(h):=\delta F(g)(h)-\lambda \delta(\mathcal{V}(g)(h)-1)=0,
$$

para todo $h \in \Gamma^{k}\left(T^{*} M \otimes T^{*} M\right)$. Calculando a variação acima, vem

$$
\delta F_{\lambda}(g) h=-\int_{M}\left\langle R i c_{g}-\frac{1}{2} R_{g} g-\frac{\lambda}{2} g, h\right\rangle_{g} \omega_{g}=0, \forall h \in \Gamma^{k}\left(T^{*} M \otimes T^{*} M\right),
$$

pelo Lema Fundamental do Cálculo das Variações, temos $R i c_{g}=u g$, onde $u=\frac{1}{2}\left(R_{g}+\lambda\right)$. Ao tomar o traço, obtemos $u=\frac{R_{g}}{m}$. Por outro lado, $\nabla_{i} u=\nabla_{i} \frac{R_{g}}{2}$, como $m \geq 3$, segue que $R_{g}$ é constante. Portanto, os pontos críticos de $F$ em $\mathcal{M}^{k}(M)_{1}$ são as métricas de Einstein de volume 1. Para obter os pontos críticos de $F$ restrito à classe conforme de volume $1,[g]_{1}$, basta tomar $h=\psi g \in T_{g}[g]$ e usar o método dos multiplicadores de Lagrange, sujeito ao vínculo $\left.\mathcal{V}\right|_{[g]} ^{-1}(1)=[g]_{1}$, para concluir que os pontos críticos de $F$ são métricas conformes a $g$, de volume 1 , e curvatura escalar constante.

Uma vez que estamos lidando com variedades com bordo minimo, consideramos

$$
\left.F\right|_{[\bar{g}]^{0}}:[\bar{g}]^{0} \longrightarrow \mathbb{R}
$$


cuja variação, para todo $h=\psi \bar{g} \in T_{\bar{g}}[\bar{g}]^{0}=\left\{\psi \in \mathcal{C}^{k, \alpha}: \partial_{\eta} \psi=0\right\}$, é dada por

$$
\begin{aligned}
\left.\delta F\right|_{[\bar{g}]^{0}}(\bar{g}) \psi \bar{g} & =-\int_{M}\left\langle R i c_{\bar{g}}-\frac{1}{2} R_{\bar{g}} \bar{g}, \psi \bar{g}\right\rangle_{\bar{g}} \omega_{\bar{g}} \\
& =-\int_{M}\left(\psi R_{\bar{g}}-\frac{1}{2} m \psi R_{\bar{g}}\right) \omega_{\bar{g}} \\
& =\frac{m-2}{2} \int_{M} \psi R_{\bar{g}} \omega_{\bar{g}} .
\end{aligned}
$$

Estamos interessados nos pontos críticos da restrição de $F$ a $\mathcal{C}^{k, \alpha}$-classe conforme normalizada de volume 1; pelo método dos multiplicadores de Lagrange, $\bar{g} \in[\bar{g}]^{0}$ é um ponto crítico do funcional $\left.F\right|_{[\bar{g}]^{0}}$ sujeito ao vínculo $[\bar{g}]_{1}^{0}=\mathcal{V}^{-1}(\{1\})$ se e somente se existe $\lambda \in \mathbb{R} \backslash\{0\}$ tal que

$$
\delta F_{\lambda}(\bar{g})(\psi \bar{g}):=\left.\delta F\right|_{[\bar{g}]^{0}}(\bar{g})(\psi \bar{g})-\lambda \delta \mathcal{V}(\bar{g})(\psi \bar{g})=0,
$$

para toda $\psi \in T_{\bar{g}}[\bar{g}]^{0}$.

A primeira variação do volume restrito à classe conforme normalizada é

$$
\left.\delta \mathcal{V}\right|_{[\bar{g}]^{0}}(\bar{g})(\psi \bar{g})=\frac{m}{2} \int_{M} \psi \omega_{\bar{g}},
$$

donde a equação (A.15) fica

$$
\begin{gathered}
\delta F_{\lambda}(\bar{g})(\psi \bar{g})=\frac{m-2}{2} \int_{M} \psi R_{\bar{g}} \omega_{\bar{g}}-\lambda \frac{m}{2} \int_{M} \psi \omega_{\bar{g}}, \\
\delta F_{\lambda}(\bar{g})(\psi \bar{g})=\frac{1}{2} \int_{M}\left[(m-2) R_{\bar{g}}-\lambda m\right] \psi \omega_{\bar{g}}
\end{gathered}
$$

para toda $\psi \in T_{\bar{g}}[\bar{g}]^{0}$.

Segue do Lema Fundamental do Cálculo das Variações que

$$
(m-2) R_{\bar{g}}-\lambda m=0 .
$$

Logo, $\bar{g}$ é ponto crítico de $F$ restrito a $[\bar{g}]_{1}^{0}$, se e só se $\bar{g} \in[\bar{g}]_{1}^{0}$ e $R_{\bar{g}}$ é constante. Além disso, vale a relação

$$
(m-2) R_{\bar{g}}=\lambda m
$$

\section{A.2 Segunda Variação do Funcional de Hilbert-Einstein}

Agora, seja $\bar{g} \in[\bar{g}]_{1}^{0}$ um ponto crítico de $F$ e considere $(\delta F)_{\bar{g}}: T_{\bar{g}}[\bar{g}]^{0} \longrightarrow \mathbb{R}$, com $T_{\bar{g}}[\bar{g}]^{0}=\left\{\psi \in \mathcal{C}^{k}(M), \partial_{\eta} \psi=0\right\}$, dada por

$$
(\delta F)_{\bar{g}}(\psi \bar{g})=\frac{m-2}{2} \int_{M} \psi R_{\bar{g}} \omega_{\bar{g}},
$$


então, a variação de $(\delta F)_{\bar{g}}(\psi \bar{g})$, fica

$$
\begin{aligned}
\delta\left((\delta F)_{\bar{g}}(\psi \bar{g})\right)(h)=\left(\delta^{2} F\right)_{\psi \bar{g}}(h) & =\frac{m-2}{2} \int_{M} \psi\left(\delta R_{\bar{g}(t)}\right) \omega_{\bar{g}}+\psi R_{\bar{g}}\left(\delta \omega_{\bar{g}(t)}\right) \\
& =\frac{m-2}{2} \int_{M} \psi\left(-h^{i j} R_{i j}+\nabla_{i}\left[\nabla_{j} h^{i j}-\nabla^{i}\left(g^{l m} h_{l m}\right)\right]\right) \omega_{\bar{g}} \\
& +\frac{m-2}{2} \int_{M} \psi R_{\bar{g}}\left(\frac{1}{2} \bar{g}^{i j} h_{i j} \omega_{\bar{g}}\right),
\end{aligned}
$$

onde $\bar{g}(t)=\bar{g}+t h, h \in \Gamma^{k}\left(T^{*} M \otimes T^{*} M\right)$. Tomando agora $h=\psi \bar{g} \in T_{\bar{g}}[\bar{g}]^{0}$ temos

$$
\begin{aligned}
\left(\delta^{2} F\right)_{\psi \bar{g}}(\psi \bar{g}) & =\frac{m-2}{2} \int_{M} \psi\left(-\psi \bar{g}^{i j} R_{i j}+\nabla_{i}\left[\nabla_{j} \psi \bar{g}^{i j}-\nabla^{i}\left(g^{l m} \psi \bar{g}_{l m}\right)\right]\right) \omega_{\bar{g}} \\
& +\frac{m-2}{2} \int_{M} \frac{1}{2} \psi R_{\bar{g}} \bar{g}^{i j} \psi \bar{g}_{i j} \omega_{\bar{g}} \\
& =\frac{m-2}{2} \int_{M} \psi\left(-\psi R_{\bar{g}}+\nabla_{i}\left[\nabla^{i} \psi-\nabla^{i}(\psi m)\right]\right) \omega_{\bar{g}} \\
& +\frac{m-2}{2} \int_{M} \frac{1}{2} \psi R_{\bar{g}} \psi m \omega_{\bar{g}} \\
& =\frac{m-2}{2} \int_{M} \psi\left(-\psi R_{\bar{g}}-\Delta_{\bar{g}} \psi+m \Delta_{\bar{g}} \psi+\frac{m}{2} \psi R_{\bar{g}}\right) \omega_{\bar{g}} \\
& =\frac{m-2}{2} \int_{M} \psi\left[(m-1) \Delta_{\bar{g}} \psi+\frac{m-2}{2} \psi R_{\bar{g}}\right] \omega_{\bar{g}},
\end{aligned}
$$

onde usamos o laplaciano geométrico: $\Delta_{\bar{g}}=-\operatorname{div}_{\bar{g}}\left(\operatorname{grad}_{\bar{g}}\right)$.

Agora, note que a segunda variação do volume fica

$$
\begin{aligned}
\left(\delta^{2} \mathcal{V}\right)_{\psi \bar{g}}(h) & =\frac{m}{2} \int_{M} \psi \delta \omega_{\bar{g}} \\
& =\frac{m}{4} \int_{M} \psi \bar{g}^{i j} h_{i j} \omega_{\bar{g}}
\end{aligned}
$$

Tomando $h=\psi \bar{g} \in T_{\bar{g}}[\bar{g}]^{0}$, temos

$$
\begin{aligned}
\left(\delta^{2} \mathcal{V}\right)_{\psi \bar{g}}(\psi \bar{g}) & =\frac{m}{4} \int_{M} \psi \bar{g}^{i j}\left(\psi \bar{g}_{i j}\right) \omega_{\bar{g}} \\
& =\frac{m^{2}}{4} \int_{M} \psi^{2} \omega_{\bar{g}}
\end{aligned}
$$

Finalmente, calculamos a segunda variação do funcional de Hilbert-Einstein sobre $[\bar{g}]_{1}^{0}$, novamente usando o método dos multiplicadores de Lagrange,

$$
\begin{gathered}
\left(\delta^{2} F_{\lambda}\right)_{\psi \bar{g}}(\psi \bar{g})=\left(\delta^{2} F\right)_{\psi \bar{g}}(\psi \bar{g})-\lambda\left(\delta^{2} \mathcal{V}\right)_{\psi \bar{g}}(\psi \bar{g}) \\
\left(\delta^{2} F_{\lambda}\right)_{\psi \bar{g}}(\psi \bar{g})=\frac{m-2}{2} \int_{M} \psi\left[(m-1) \Delta_{\bar{g}} \psi+\frac{m-2}{2} \psi R_{\bar{g}}\right] \omega_{\bar{g}}-\lambda \frac{m^{2}}{4} \int_{M} \psi^{2} \omega_{\bar{g}}
\end{gathered}
$$

que fica

$$
\left(\delta^{2} F_{\lambda}\right)_{\psi \bar{g}}(\psi \bar{g})=\frac{m-2}{2} \int_{M} \psi\left[(m-1) \Delta_{\bar{g}} \psi\right] \omega_{\bar{g}}+\int_{M}\left(\frac{(m-2)^{2}}{4} R_{\bar{g}}-\frac{\lambda m^{2}}{4}\right) \psi^{2} \omega_{\bar{g}},
$$


Usando (A.16), vemos que

$\frac{(m-2)^{2}}{4} R_{\bar{g}}-\frac{\lambda m^{2}}{4}=\frac{(m-2)^{2} R_{\bar{g}}-(m-2) R_{\bar{g}} m}{4}=\frac{(m-2)}{2}\left[\frac{m-2-m}{2} R_{\bar{g}}\right]=\frac{(m-2)}{2}\left(-R_{\bar{g}}\right)$.

Portanto, a segunda variação de $F$, restrito à $[\bar{g}]_{1}^{0}$, é dada pela forma quadrática

$$
\delta^{2} F(\bar{g})(\psi)=\frac{m-2}{2} \int_{M}\left((m-1) \Delta_{\bar{g}} \psi-R_{\bar{g}} \psi\right) \psi \omega_{\bar{g}},
$$

onde $\psi \in \mathcal{C}^{k, \alpha}(M)^{0}$ e tem integral nula (veja também [26]).

\section{Classificação de Pontos Críticos}

Um ponto crítico $\bar{g}$ de $F$, é dito não degenerado se a forma quadrática $\delta^{2} F(\bar{g})(\psi)$ é não degenerada. Lembremos que um forma quadrática $Q(x)$ em um espaço vetorial $V$ é não degenerada se a forma bilinear à ela associada,

$$
B(x, y)=Q(x+y)-Q(x)-Q(y)
$$

é não degenerada, isto é, se as aplicações

$$
x \mapsto(y \mapsto B(x, y)) \quad \text { e } \quad y \mapsto(x \mapsto B(x, y))
$$

são isomorfismos entre $V$ e seu dual $V^{*}$.

A forma bilinear associada à $\delta^{2} F(\bar{g})(\psi)$ é dada por

$$
\delta^{2} F(\bar{g})(\psi, \varphi)=\frac{m-2}{2} \int_{M}\left((m-1) \Delta_{\bar{g}} \psi-R_{\bar{g}} \psi\right) \varphi \omega_{\bar{g}}
$$

onde $\varphi, \psi \in \mathcal{C}^{k, \alpha}(M)$ têm derivada normal nula e integral sobre $M$ nula.

Note que $(m-1) \Delta_{g}-R_{g} I$, leva o subespaço de $\mathcal{C}^{k, \alpha}(M)$,

$$
T_{\bar{g}}[\bar{g}]_{1}^{0}=\left\{\psi \in \mathcal{C}^{k, \alpha}(M): \partial_{\eta_{\bar{g}}} \psi=0, \int_{M} \psi \omega_{\bar{g}}=0\right\}
$$

no subespaço de $\mathcal{C}^{k-2, \alpha}(M)$

$$
\mathcal{C}_{0}^{k-2, \alpha}(M)=\left\{\varphi \in \mathcal{C}^{k-2, \alpha}(M): \int_{M} \varphi \omega_{\bar{g}}=0\right\}
$$

De fato, para toda $\psi \in T_{\bar{g}}[\bar{g}]_{1}^{0}$, temos

$$
\begin{aligned}
\int_{M}(m-1) \Delta_{\bar{g}} \psi-R_{\bar{g}} \psi \omega_{\bar{g}} & =(m-1) \int_{M} \Delta_{\bar{g}} \psi \omega_{\bar{g}}-R_{\bar{g}} \int_{M} \psi \omega_{\bar{g}} \\
& =(m-1) \int_{\partial M}\left\langle\nabla \psi, \eta_{\bar{g}}\right\rangle \sigma_{\bar{g}} \\
& =(m-1) \int_{\partial M} \partial_{\eta_{\bar{g}}} \psi \sigma_{\bar{g}} \\
& =0
\end{aligned}
$$

Assim, se para toda $\psi \in T_{\bar{g}}[\bar{g}]_{1}^{0}$, temos $\delta^{2} F(\bar{g})(\psi, \varphi)=0$, então, pelo Lema Fundamental do Cálculo das Variações, $\varphi$ é constante. Mas, $\int_{M} \varphi \omega_{\bar{g}}=0$, $\log 0 \varphi=0$. 
Por outro lado, suponha que $\delta^{2} F(\bar{g})(\psi, \varphi)=0$ para toda $\varphi \in T_{\bar{g}}[\bar{g}]_{1}^{0}$,

$$
\frac{m-2}{2} \int_{M}\left((m-1) \Delta_{\bar{g}} \psi-R_{\bar{g}} \psi\right) \varphi \omega_{\bar{g}}=0,
$$

então, pelo Lema Fundamental do Cálculo das Variações, segue que $(m-1) \Delta_{\bar{g}} \psi-R_{\bar{g}} \psi$ é constante. Mas, vimos acima, que

$$
\int_{M}(m-1) \Delta_{\bar{g}} \psi-R_{\bar{g}} \psi \omega_{\bar{g}}=0
$$

Logo, $(m-1) \Delta_{\bar{g}} \psi-R_{\bar{g}} \psi=0$, que implica em $\Delta_{\bar{g}} \psi=\frac{R_{\bar{g}}}{m-1} \psi$, com $\partial_{\eta} \psi=0$ portanto $\psi=0$ se e somente se

$$
R_{\bar{g}}=0 \text { ou } \frac{R_{\bar{g}}}{m-1} \text { não é solução do problema de Neumann }\left\{\begin{array}{rl}
\Delta_{\bar{g}} \psi & =\lambda \psi, \text { em } M \\
\partial_{\eta} \psi & =0, \text { em } \partial M
\end{array} .\right.
$$

De fato, se $\frac{R_{\bar{g}}}{m-1}$ não é autovalor de $\Delta_{\bar{g}}$, não pode existir $\psi \neq 0$ tal que

$$
\Delta_{\bar{g}} \psi=\frac{R_{\bar{g}}}{m-1} \psi
$$

donde $\psi=0$. Se, por outro lado, $R_{\bar{g}}=0$, temos $\Delta_{\bar{g}} \psi=0$. Como $\partial_{\eta} \psi=0$, segue do Teorema da Divergência que

$$
\int_{M}\langle\nabla \psi, \nabla \psi\rangle_{\bar{g}} \omega_{\bar{g}}=\int_{M} \psi \Delta \psi \omega_{\bar{g}}-\int_{\partial M} \psi \partial_{\eta} \psi \sigma_{\bar{g}}=0
$$

isto é, $\|\nabla \psi\|^{2}=0$, portanto $\psi$ é constante. Uma vez que $\psi$ tem integral nula sobre $M$, temos que $\psi=0$. Assim, sob essas condições, $\Delta_{\bar{g}}-R_{\bar{g}} I$ é injetor.

Para provarmos a sobrejetividade, basta observarmos que, de $T_{\bar{g}}[\bar{g}]_{1}^{0}$ em $\mathcal{C}_{0}^{k-2, \alpha}(M)$, o operador $\Delta_{\bar{g}}-R_{\bar{g}} I$ é de Fredholm de índice zero ${ }^{1}$ (ver Seção 2), portanto injetividade implica em sobrejetividade.

Com efeito, é um fato conhecido da Análise que o operador Laplaciano é de Fredholm de índice zero entre os espaços de Hölder

$$
\mathcal{C}^{k, \alpha}(M) \stackrel{\Delta_{\bar{g}}}{\longrightarrow} \mathcal{C}^{k-2, \alpha}(M)
$$

então, considere a composição

$$
T_{\bar{g}}[\bar{g}]_{1}^{0} \stackrel{i}{\longrightarrow} \mathcal{C}^{k, \alpha}(M) \stackrel{\Delta_{\bar{g}}}{\longrightarrow} \mathcal{C}^{k-2, \alpha}(M) \stackrel{p}{\longrightarrow} \mathcal{C}_{0}^{k-2, \alpha}(M),
$$

onde $i$ é a inclusão e $p$ é a projeção. Daí,

- $\operatorname{dim} \operatorname{ker}(i)=0, \operatorname{codim} \operatorname{Im}(i)=1 \Rightarrow \operatorname{ind}(i)=-1$,

- $\operatorname{dim} \operatorname{ker}(p)=1, \operatorname{codim} \operatorname{Im}(p)=0 \Rightarrow \operatorname{ind}(i)=1$,

onde ind denota o índice de Fredholm do operador. Isso prova que $\Delta_{\bar{g}}$ é de Fredholm de índice zero de $T_{\bar{g}}[\bar{g}]_{1}^{0}$ em $\mathcal{C}_{0}^{k-2, \alpha}(M)$. Além disso, $k+\alpha>(k-2)+\alpha$ implica que a inclusão $\mathcal{C}^{k, \alpha}(M) \stackrel{i}{\hookrightarrow} \mathcal{C}^{k-2, \alpha}(M)$ é compacta (ver Proposição 2.2.9), donde o operador $R_{\bar{g}} I: T_{\bar{g}}[\bar{g}]_{1}^{0} \longrightarrow$ $\mathcal{C}_{0}^{k-2, \alpha}(M)$, que pode ser descrito como

$$
T_{\bar{g}}[\bar{g}]_{1}^{0} \stackrel{R_{\bar{g}} I}{\longrightarrow} T_{\bar{g}}[\bar{g}]^{0} \stackrel{i}{\longleftrightarrow} \mathcal{C}^{k, \alpha}(M) \stackrel{i}{\longrightarrow} \mathcal{C}^{k-2, \alpha}(M) \stackrel{p}{\longrightarrow} \mathcal{C}_{0}^{k-2, \alpha}(M),
$$

\footnotetext{
${ }^{1}$ Daí o motivo de considerarmos espaços de Hölder.
} 
é compacto, portanto

$$
(m-1) \Delta_{\bar{g}}-R_{\bar{g}} I: T_{\bar{g}}[\bar{g}]_{1} \longrightarrow \mathcal{C}_{0}^{k-2, \alpha}(M)
$$

é de Fredholm de índice zero, como queríamos. Segue que a forma quadrática $\delta^{2} F(\bar{g})(\psi \bar{g}, \psi \bar{g})$ é não degenerada se e só se $\operatorname{ker}\left((m-1) \Delta_{g}-R_{g} I\right)=\{0\}$, ou seja, se $R_{g}=0$ ou se $\frac{R_{\bar{g}}}{m-1}$ não é solução do problema de Neumann

$$
\left\{\begin{aligned}
\Delta_{\bar{g}} \psi & =\rho \psi, \text { on } M \\
\partial_{\eta_{\bar{g}}} \psi & =0, \text { on } \partial M .
\end{aligned}\right.
$$


APÊNDICE A 


\section{Apêndice B}

\section{Funções com Derivada Normal Prescrita}

Seja $n \geq 1$ e seja $g: \mathbb{R}^{n} \longrightarrow \mathbb{R}$ uma função contínua. Denotaremos por $\left(x_{1}, \ldots, x_{n}\right)$ as coordenadas no $\mathbb{R}^{n}$ e por $\left(x_{1}, \ldots, x_{n}, z\right)$ as coordenadas no $\mathbb{R}^{n+1}$. Definimos

$$
F_{g}: \mathbb{R}^{n} \longrightarrow \mathbb{R}
$$

por

$$
F_{g}\left(x_{1}, x_{2}, \ldots, x_{n}, z\right)=\frac{1}{z^{n-1}} \int_{x_{n}-\frac{1}{2} z}^{x_{n}+\frac{1}{2} z} \ldots \int_{x_{2}-\frac{1}{2} z}^{x_{2}+\frac{1}{2} z} \int_{x_{1}-\frac{1}{2} z}^{x_{1}+\frac{1}{2} z} g\left(s_{1}, s_{2}, \ldots, s_{n}\right) d s_{1} d s_{2} \ldots d s_{n},
$$

se $z \neq 0$, e

$$
F_{g}\left(x_{1}, x_{2}, \ldots, x_{n}, 0\right)=0 .
$$

Pela linearidade da integral, $F_{\lambda g+h}=\lambda F_{g}+F_{h}$, para todo $\lambda \in \mathbb{R}$ e toda $g, h \in \mathcal{C}^{0}\left(\mathbb{R}^{n}\right)$, além disso, $F_{g}$ é de classe $\mathcal{C}^{1}$ em $\mathbb{R}^{n+1} \backslash\{z=0\}$.

A derivada parcial de $F_{g}$ é com respeito a $z$, no ponto $\left(x_{1}, x_{2}, \ldots, x_{n}, 0\right)$ é dada por

$$
\begin{aligned}
\frac{\partial}{\partial z} F_{g}\left(x_{1}, x_{2}, \ldots, x_{n}, 0\right) & =\lim _{z \rightarrow 0} \frac{1}{z} F_{g}\left(x_{1}, x_{2}, \ldots, x_{n}, z\right) \\
& =\lim _{z \rightarrow 0}\left[\frac{1}{z^{n}} \int_{x_{n}-\frac{1}{2} z}^{x_{n}+\frac{1}{2} z} \cdots \int_{x_{2}-\frac{1}{2} z}^{x_{2}+\frac{1}{2} z} \int_{x_{1}-\frac{1}{2} z}^{x_{1}+\frac{1}{2} z} g\left(s_{1}, s_{2}, \ldots, s_{n}\right) d s_{1} d s_{2} \ldots d s_{n}\right] \\
& =g\left(x_{1}, x_{2}, \ldots, x_{n}\right),
\end{aligned}
$$

todas as demais derivadas se anulam

$$
\frac{\partial}{\partial x_{i}} F_{g}\left(x_{1}, x_{2}, \ldots, x_{n}, 0\right)=0, i=1,2, \ldots, n \text {. }
$$

Afirmação B.0.1. $F_{g}$ é de classe $\mathcal{C}^{1}$ no $\mathbb{R}^{n+1}$.

Demonstração. A prova é por indução sobre $n$. O caso $n=1$ é claro (não há singularidade neste caso). É conveniente adotarmos a seguinte notação: dado $n \geq 2, i \in\{1, \ldots, n\}$ e $s \in \mathbb{R}$, seja $g^{(i, s)}$ a função contínua definida no $\mathbb{R}^{n-1}$, tomando valores em $\mathbb{R}^{n}$, definida por

$$
g^{(i, s)}\left(x_{1}, \ldots, x_{n-1}\right)=g\left(x_{1}, \ldots, x_{i-1}, s, x_{i}, \ldots, x_{n-1}\right) .
$$


Primeiro, vamos estabelecer a continuidade de $\frac{\partial}{\partial z} F_{g}$. Um cálculo direto, mostra que, para todo $i$ e $z \neq 0$, a seguinte igualdade vale:

$$
\begin{aligned}
\frac{1}{2} F_{g^{\left(i, x_{i}+z / 2\right)}}\left(x_{1}, \ldots, \widehat{x^{i}}, \ldots,\right. & \left.x_{n}, z\right)+\frac{1}{2} F_{g^{\left(i, x_{i}-z / 2\right)}}\left(x_{1}, \ldots, \widehat{x^{i}}, \ldots, x_{n}, z\right) \\
& +\int_{x_{i}-\frac{1}{2} z}^{x_{i}+\frac{1}{2} z} \frac{\partial}{\partial z} F_{g}^{(i, s)}\left(x_{1}, \ldots, \widehat{x^{i}}, \ldots, x_{n}, z\right) d s \\
= & F_{g}\left(x_{1}, \ldots, x_{n}, z\right)+z \frac{\partial}{\partial z} F_{g}\left(x_{1}, \ldots, x_{n}, z\right),
\end{aligned}
$$

onde $\widehat{x_{i}}$ significa que estamos omitindo a coordenada $x_{i}$. Calculando diretamente da definição de $F_{g}$, temos

$$
\begin{aligned}
\frac{\partial F_{g}}{\partial z}\left(x_{1}, \ldots, x_{n}, z\right) & =-\frac{n-1}{z} F_{g}\left(x_{1}, \ldots, x_{n}, z\right) \\
& +\frac{1}{2 z} \sum_{i=1}^{n}\left[F_{g^{\left(i, x_{i}+z / 2\right)}}\left(x_{1}, \ldots, \widehat{x_{i}}, \ldots, x_{n}, z\right)+F_{g^{\left(i, x_{i}-z / 2\right)}}\left(x_{1}, \ldots, \widehat{x_{i}}, \ldots, x_{n}, z\right)\right],
\end{aligned}
$$

e usando (B.3), obtemos

$$
\begin{aligned}
\frac{\partial F_{g}}{\partial z}\left(x_{1}, \ldots, x_{n}, z\right) & =-\frac{n-1}{z} F_{g}\left(x_{1}, \ldots, x_{n}, z\right) \\
& +\frac{n}{z} F_{g}\left(x_{1}, \ldots, x_{n}, z\right)+n \frac{\partial F_{g}}{\partial z}\left(x_{1}, \ldots, x_{n}, z\right) \\
& -\frac{1}{z} \sum_{i=1}^{n} \int_{x_{i}-\frac{z}{2}}^{x_{i}+\frac{z}{2}} \frac{\partial F_{g}(i, s)}{\partial z}\left(x_{1}, \ldots, \widehat{x}_{i}, \ldots, x_{n}, z\right) d s
\end{aligned}
$$

isto é,

$$
\begin{aligned}
(1-n) \frac{\partial F_{g}}{\partial z}\left(x_{1}, \ldots, x_{n}, z\right) & =\frac{1}{z} F_{g}\left(x_{1}, \ldots, x_{n}, z\right) \\
& -\frac{1}{z} \sum_{i=1}^{n} \int_{x_{i}-z / 2}^{x_{i}+z / 2} \frac{\partial F_{g}^{(i, s)}}{\partial z}\left(x_{i}, \ldots, \widehat{x_{i}}, \ldots, x_{n}, z\right) d s .
\end{aligned}
$$

Vamos agora tomar o limite quando $\left(x_{1}, \ldots, x_{n}, z\right) \rightarrow\left(\bar{x}_{1}, \ldots, \bar{x}_{n}, 0\right)$ em ambos os lados de (B.4). Pela hipótese de indução,

$$
\lim _{\left(x_{1}, \ldots, x_{n}, z\right) \rightarrow\left(\bar{x}_{1}, \ldots, \bar{x}_{n}, 0\right)} \frac{\partial F_{g^{(i, s)}}}{\partial z}\left(x_{1}, \ldots, \widehat{x}_{i}, \ldots, x_{n}, z\right)=g^{(i, s)}\left(\bar{x}_{1}, \ldots, \widehat{\bar{x}}_{i}, \ldots, \bar{x}_{n}\right),
$$

o que nos dá

$$
\begin{aligned}
\lim _{\left(x_{1}, \ldots, x_{n}, z\right) \rightarrow\left(\bar{x}_{1}, \ldots, \bar{x}_{n}, 0\right)} \frac{1}{z} \int_{x_{i}-\frac{z}{2}}^{x_{i}+\frac{z}{2}} \frac{\partial F_{g^{(i, s)}}}{\partial z}\left(x_{1}, \ldots, \widehat{x}_{i}, \ldots, x_{n}, z\right) d s & =g^{\left(i, \bar{x}_{i}\right)}\left(\bar{x}_{1}, \ldots, \widehat{\bar{x}}_{i}, \ldots, \bar{x}_{n}\right) \\
& =g\left(\bar{x}_{1}, \ldots, \bar{x}_{n}\right) .
\end{aligned}
$$

De (B.2), (B.4) e (B.5), finalmente obtemos

$$
(1-n) \lim _{\left(x_{1}, \ldots, x_{n}, z\right) \rightarrow\left(\bar{x}_{1}, \ldots, \bar{x}_{n}, 0\right)} \frac{\partial F_{g}}{\partial z}\left(x_{1}, \ldots, x_{n}, z\right)=(1-n) g\left(\bar{x}_{1}, \ldots, \bar{x}_{n}\right),
$$

que nos dá a continuidade de $\frac{\partial F_{g}}{\partial z}$ em $\mathbb{R}^{n+1}$. Para provar a continuidade das outras derivadas 
parciais $\frac{\partial F_{g}}{\partial x_{i}}$ em $\{z=0\}$, primeiro observamos que para $n=1$ é um resultado óbvio. Para o caso $n \geq 2$, escrevemos expressão análoga à (B.3):

$$
\begin{aligned}
\frac{\partial F_{g}}{\partial x_{i}}\left(x_{1}, \ldots, x_{n}, z\right) & = \\
& =\frac{1}{z}\left[F_{g^{\left(i, x_{i}+z / 2\right)}}\left(x_{1}, \ldots, \widehat{x^{i}}, \ldots, x_{n}, z\right)-F_{g^{\left(i, x_{i}-z / 2\right)}}\left(x_{1}, \ldots, \widehat{x_{i}}, \ldots, x_{n}, z\right)\right]
\end{aligned}
$$

que vale para todo $n \geq 2, i=1, \ldots, n$, e $z \neq 0$. Usando o Teorema do Valor Médio, concluímos que

$$
\begin{aligned}
\lim _{\left(x_{1}, \ldots, x_{n}, z\right) \rightarrow\left(\bar{x}_{1}, \ldots, \bar{x}_{n}, 0\right)} \frac{1}{z} & F_{g^{\left(i, x_{i}+z / 2\right)}}\left(x_{1}, \ldots, \widehat{x}_{i}, \ldots x_{n}, z\right)= \\
& =\lim _{\left(x_{1}, \ldots, x_{n}, z\right) \rightarrow\left(\bar{x}_{1}, \ldots, \bar{x}_{n}, 0\right)} \frac{1}{z} F_{g^{\left(i, x_{i}-z / 2\right)}}\left(x_{1}, \ldots, \widehat{x_{i}}, \ldots x_{n}, z\right) \\
& =g\left(\bar{x}_{1}, \ldots, \bar{x}_{n}\right),
\end{aligned}
$$

que nos dá

$$
\lim _{\left(x_{1}, \ldots, x_{n}, z\right) \rightarrow\left(\bar{x}_{1}, \ldots, \bar{x}_{n}, 0\right)} \frac{\partial F_{g}}{\partial x_{i}}\left(x_{1}, \ldots, x_{n}, z\right)=0,
$$

isto é, a continuidade de $\frac{\partial F_{g}}{\partial x_{i}}$.

Afirmação B.0.2. Se $g$ é de classe $\mathcal{C}^{k}$, com $k \geq 1$, então $F_{g}$ é de classe $\mathcal{C}^{k+1}$.

Demonstração. Calculando sua derivada, diretamente da definição, vemos que $F_{g}$, restrita a $\{z \neq 0\}$ é de classe $\mathcal{C}^{k+1}$. Assim, vamos estudar a regularidade de $F_{g}$ no hiperplano $\{z=0\}$. Usando a notação de multi-índice, $\mathbf{j}=\left(j_{1}, \ldots, j_{n}\right),|\mathbf{j}|=j_{1}+\ldots+j_{n}, \mathbf{x}=\left(x_{1}, \ldots, x_{n}\right)$, escrevemos

$$
g(\mathbf{x})=\sum_{|\mathbf{j}| \leq k} \frac{1}{|\mathbf{j}| !} \frac{\partial^{\mathbf{j}} g}{\partial \mathbf{x}^{\mathbf{j}}}(\overline{\mathbf{x}})(\mathbf{x}-\overline{\mathbf{x}})^{\mathbf{j}}+R(\mathbf{x}, \overline{\mathbf{x}})
$$

onde $R(\mathbf{x}, \overline{\mathbf{x}})$ é uma função contínua em $\mathbb{R}^{2 n}$, satisfazendo

$$
|R(\mathbf{x}, \overline{\mathbf{x}})|=\mathrm{o}\left(\mid \mathbf{x}-\overline{\mathbf{x}}^{k}\right), \text { onde } \mathbf{x} \rightarrow \overline{\mathbf{x}} .
$$

Aqui, temos

$$
\frac{\partial^{\mathbf{j}} g}{\partial \mathbf{x}^{\mathbf{j}}}=\frac{\partial^{|\mathbf{j}|} g}{\partial x_{1}^{j_{1}} \ldots \partial x_{n}^{j_{n}}} \text { e }(\mathbf{x}-\overline{\mathbf{x}})^{\mathbf{j}}=\left(x_{1}-\bar{x}_{1}\right)^{j_{1}} \ldots\left(x_{n}-\bar{x}_{n}\right)^{j_{n}} .
$$

Assim, a expressão para $F_{g}$ fica

$$
F_{g}(\mathbf{x}, z)=\frac{1}{z^{n-1}} \sum_{|\mathbf{j}| \leq k} \frac{1}{|\mathbf{j}| !} \frac{\partial^{\mathbf{j}} g}{\partial \mathbf{x}^{\mathbf{j}}}(\overline{\mathbf{x}}) \int_{Q_{n}(\mathbf{x}, z)}(\mathbf{s}-\overline{\mathbf{x}})^{\mathbf{j}} d \mathbf{s}+\frac{1}{z^{n-1}} \int_{Q_{n}(\mathbf{x}, z)} R(\mathbf{s}, \overline{\mathbf{x}}) d \mathbf{s}
$$

onde $Q_{n}(\mathbf{x}, z)$ é o $n$-cubo $\prod_{j=1}^{n}\left[x_{j}-\frac{z}{2}, x_{j}+\frac{z}{2}\right]$. Agora é fácil ver que cada integral $\frac{1}{z^{n-1}} \int_{Q_{n}(\mathbf{x}, z)}(\mathbf{s}-\overline{\mathbf{x}})^{\mathbf{j}} d \mathbf{s}$ é um monômio de grau $|\mathbf{j}|+1$ em $\left(x_{1}-\bar{x}_{1}\right), \ldots,\left(x_{n}-\bar{x}_{n}\right)$ e $z$. Mais precisamente,

$$
\frac{1}{z^{n-1}} \int_{Q_{n}(\mathbf{x}, s)}(\mathbf{s}-\overline{\mathbf{x}})^{\mathbf{j}} d \mathbf{s}=z \cdot \prod_{i=1}^{n}\left[\frac{1}{z} \int_{x_{i}-\frac{z}{2}}^{x_{i}+\frac{z}{2}}\left(s-\bar{x}_{i}\right)^{j_{i}} d s\right]
$$




$$
\frac{1}{z} \int_{x_{i}-\frac{z}{2}}^{x_{i}+\frac{z}{2}}\left(s-\bar{x}_{i}\right)^{j_{i}} d s=\frac{1}{j_{i}+1} \sum_{\ell=0}^{\left[j_{i} / 2\right]} \frac{1}{2^{2 \ell}}\left(\begin{array}{c}
j_{i}+1 \\
2 \ell+1
\end{array}\right) z^{2 \ell}\left(x_{i}-\bar{x}_{i}\right)^{j_{i}-2 \ell}
$$

onde $\left[j_{1} / 2\right]$ é a parte inteira de $j_{i} / 2$. Agora, usando o Teorema do Valor Médio para integrais, é fácil ver que a função contínua

$$
\widetilde{R}(\mathbf{x}, \overline{\mathbf{x}}, z)=\frac{1}{z^{n-1}} \int_{Q_{n}(\mathbf{x}, z)} R(\mathbf{s}, \overline{\mathbf{x}}) d \mathbf{s}
$$

satisfaz

$$
|\widetilde{R}(\mathbf{x}, \overline{\mathbf{x}}, z)|=\mathrm{o}\left(\left(|\mathbf{x}-\overline{\mathbf{x}}|^{2}+z^{2}\right)^{\frac{k+1}{2}}\right) \text {, onde }(\mathbf{x}, z) \rightarrow(\overline{\mathbf{x}}, 0) .
$$

A conclusão é que (B.6) dá a fórmula de Taylor de ordem $k+1$ para a função $F_{g}$ em todos os pontos de $\mathbb{R}^{n} \times\{0\}$ e, em particular, prova que $F_{g}$ é de classe $\mathcal{C}^{k+1}$ em $\mathbb{R}^{n+1}$.

Observação B.0.3. Usando indução, é fácil mostrar que, se g é de classe $\mathcal{C}^{k}$, para todo $j \leq k$, as derivadas parciais de ordem $j$ de $F_{g}$ são combinações lineares, integrais e composições das funções lineares nas variáveis das derivadas parciais de ordem $j-1$ da função $g$. Isto também é visível na fórmula de Taylor de ordem $k+1$ de $F_{g}$, dada em (B.6), envolvendo derivadas de $g$ de ordens maiores que $k$.

Vamos agora considerar a situação na qual estamos realmente interessados. Seja $\Sigma^{n-1}$ uma variedade compacta com bordo suave $\partial \Sigma$; seja $\eta$ uma campo vetorial suave ao longo de $\partial \Sigma$ que é transversal ao bordo em todo ponto. Fixe $k \geq 0$ e $\alpha \in(0,1]$.

Proposição B.0.4. Existe uma aplicação linear contínua

$$
\mathcal{F}: \mathcal{C}^{k, \alpha}(\partial \Sigma) \longrightarrow \mathcal{C}^{k+1, \alpha}(\Sigma)
$$

tal que, para todo $\tilde{g} \in \mathcal{C}^{k, \alpha}(\partial \Sigma)$ as seguintes propriedades são satisfeitas:

(i.) $\mathcal{F}(\tilde{g})$ se anula em $\partial \Sigma$;

(ii.) $\eta(\mathcal{F}(\tilde{g}))=\tilde{g}$.

Demonstração. Escolha um conjunto finito de cartas locais $\left(U_{r}, \phi_{r}\right), r=1, \ldots, N$ em $\Sigma$ satisfazendo as seguintes propriedades:

(a.) $U_{r}$ é um subconjunto de $\Sigma$, com $U_{r} \cap \partial \Sigma \neq \emptyset$ para todo $r$;

(b.) $U:=\cup_{r=1}^{N} U_{r}$ é uma vizinhança aberta do bordo $\partial \Sigma$;

(c.) $\phi_{r}$ é um difeomorfismo de $U_{r}$ para $\mathbb{R}^{n} \times[0, \infty)$ que leva $\partial \Sigma \cap U_{r}$ sobre $\mathbb{R} \times\{0\}$;

(d.) $\left(\mathrm{d} \phi_{r}\right)_{p}\left(\eta_{p}\right)=\frac{\partial}{\partial z}$, para todo $p \in \partial \Sigma \cap U_{r}$.

Seja $U_{0}=\Sigma \backslash \partial \Sigma$, tal que $\left\{U_{r}\right\}_{r=0, \ldots, N}$ é uma cobertura aberta de $\Sigma$, e seja $\left\{\rho_{r}\right\}_{r=0}^{N}$ uma partição suave da unidade subordinada a esta cobertura. Neste contexto, a função desejada, $\mathcal{F}$, é definida como segue.

Dada $\tilde{g} \in \mathcal{C}^{k, \alpha}(\partial \Sigma)$, para todo $r$, considere a função $g_{r}=\tilde{g} \circ \phi_{r}^{-1}: \mathbb{R}^{n} \longrightarrow \mathbb{R}$, que é de classe $\mathcal{C}^{k, \alpha}$. Seja $F_{g_{r}}: \mathcal{R}^{n+1} \longrightarrow \mathbb{R}$ a $\mathcal{C}^{k+1, \alpha_{-}}$extensão de $g_{r}$ definida pela fórmula (B.1), e defina $\widetilde{F}_{r}=F_{g_{r}} \circ \phi_{r}$ a aplicação $\mathcal{C}^{k+1, \alpha}$ em $U_{r}$. Finalmente, defina

$$
\mathcal{F}(\tilde{g})=\sum_{r=1}^{N} \phi_{r} \cdot \tilde{F}_{r}
$$


a função $\mathcal{C}^{k+1, \alpha}$ em $\Sigma$.

Vamos verificar que $\mathcal{F}(\tilde{g})$ tem as características desejadas. Claramente, $\mathcal{F}(\tilde{g})$ depende linearmente de $\tilde{g}$. A continuidade de $\mathcal{F}$ segue da Observação B.0.3. Como todas as $F_{r}$ se anulam em $\mathbb{R}^{n} \times\{0\}$, por $(c$. $)$, todas as $\widetilde{F}_{r}$ se anulam no bordo, portanto $\mathcal{F}(g)$ se anula em $\partial \Sigma$. Uma vez que

$$
\frac{\partial F_{g_{r}}}{\partial z}\left(x_{1}, \ldots, x_{n}, 0\right)=g_{r}\left(x_{1}, \ldots, x_{n}\right),
$$

por $(d$.$) , temos \eta_{p}\left(\widetilde{F}_{r}\right)=\tilde{g}(p)$ para todo $p \in \partial \Sigma \cap U_{r}$. Por outro lado, se $p \in \partial \Sigma \backslash U_{r}$, então $\phi_{r}(p)=0$. Portanto, para $p \in \partial \Sigma$,

$$
\eta_{p}(\mathcal{F}(g))=\sum_{r=1}^{N}\left[\eta_{p}\left(\phi_{r}\right) \cdot \widetilde{F}_{r}(p)+\phi_{r}(p) \eta_{p}\left(\widetilde{F}_{r}\right)\right]=\sum_{r=1}^{N} \phi_{r}(p) g(p)=g(p) ;
$$

o que conclui a prova.

Corolário B.0.5. Para todo $k \geq 0$ e $\alpha \in(0,1]$, a aplicação linear limitada

$$
\begin{aligned}
\mathcal{L}: \mathcal{C}^{k_{1}, \alpha}(\Sigma) & \longrightarrow \mathcal{C}^{k+1, \alpha}(\partial \Sigma) \times \mathcal{C}^{k, \alpha}(\partial \Sigma) \\
F & \longmapsto\left(\left.F\right|_{\partial \Sigma}, \eta(F)\right)
\end{aligned}
$$

admite um inverso à direita.

Demonstração. Assuma que o campo $\eta$ aponta para dentro de $\partial \Sigma$. Dada $h \in \mathcal{C}^{k+1, \alpha}(\partial \Sigma)$, construa uma função $\mathcal{E}(h) \in \mathcal{C}^{k+1}(\Sigma)$ com as seguintes propriedades

- $\left.\mathcal{E}(h)\right|_{\partial \Sigma}=h$;

- $\eta(\mathcal{E}(h)) \equiv 0$.

Para isso, considere a métrica riemanniana em $\Sigma$ tal que $\eta$ é um campo vetorial ao longo de $\partial \Sigma$ que é ortogonal a $\partial \Sigma$; seja exp a aplicação exponencial a ela associada. Uma vizinhança apropriada $U$ de $\partial \Sigma$ pode ser identificada com o produto $\partial \Sigma \times[0, \varepsilon)$ via o difeomorfismo

$$
\begin{aligned}
\partial \Sigma \times[0, \varepsilon) & \longrightarrow U \\
(p, t) & \longmapsto \exp _{p}\left(t \cdot \eta_{p}\right),
\end{aligned}
$$

onde $\varepsilon>0$ é um número suficientemente pequeno. Uma aplicação $\tilde{h} \in U$ pode ser definida em $U$ pondo $\tilde{h}(p, t)=h(p)$, para todo $(p, t) \in \partial \Sigma \times[0, \varepsilon)$. Escolha uma função suave $\Xi: \Sigma \longrightarrow[0,1]$ que é identicamente $1 \mathrm{em}$ uma vizinhança de $\partial \Sigma$ e cujo suporte está contido em $U$. Então, $\mathcal{E}(h): \mathcal{C}^{k+1}(\partial \Sigma) \longrightarrow \mathcal{C}^{k+1}(\Sigma)$ é uma aplicação linear limitada. Uma inversa à direita $\mathcal{R}$ para $\mathcal{L}$ é dada por

$$
\mathcal{R}(h, g)=\mathcal{E}(h)+\mathcal{F}(g) .
$$

Corolário B.0.6. Para todo $k \geq 1$ e para todo $\alpha \in(0,1]$, o subespaço

$$
\mathcal{C}_{\eta}^{k, \alpha}(\Sigma)=\left\{F \in \mathcal{C}^{k, \alpha}(\Sigma): \eta(F) \equiv 0\right\}
$$

é fechado e complementado em $\mathcal{C}^{k, \alpha}(\Sigma)$.

Demonstração. O conjunto $\mathcal{C}_{\eta}^{k, \alpha}(\Sigma)$ é claramente fechado na topologia $\mathcal{C}^{1}$. A propriedade de ser complementado é equivalente a existência de um inverso limitado à direito para a aplicação

$$
\begin{aligned}
\mathcal{C}^{k, \alpha}(\Sigma) & \longrightarrow \mathcal{C}^{k-1, \alpha}(\partial \Sigma) \\
F & \longmapsto \eta(F),
\end{aligned}
$$


Tal inverso à direita é dado por $g \longmapsto \mathcal{R}(0, g)$, onde $\mathcal{R}$ foi definida no corolário anterior. 


\section{Apêndice C}

\section{G-equivariância da Curvatura Escalar}

Seja $M$ uma variedade riemanniana compacta e seja $\left\{g_{s}\right\}_{s \in[a, b]}$ uma família de métricas de volume 1 e curvatura escalar constante. Seja $G$ um grupo de Lie conexo agindo em $M$ por difeomorfismos que preservam $g_{s}$ para todo $s$. Queremos mostrar que para toda $\phi \in \mathcal{C}^{k, \alpha}(M)$, positiva e para todo difeomorfismo $\xi \in G$, temos

$$
R_{(\phi \circ \xi) g_{s}}=R_{\phi g_{s}} \circ \xi .
$$

Se $\tilde{g}_{s}=\mathrm{e}^{2 f} g_{s}$, sabemos que

$$
R_{\tilde{g}_{s}}=\mathrm{e}^{-2 f}\left[R_{g_{s}}+2(m-1) \Delta f-(m-2)(m-1)\|\nabla f\|^{2}\right] .
$$

Fazendo $\mathrm{e}^{2 f}=\phi$, temos

$$
f=\frac{1}{2} \ln \phi, \quad \nabla f=\frac{1}{2} \frac{\nabla \phi}{\phi}, \quad \Delta f=\frac{1}{2}\left(\frac{\Delta \phi}{\phi}+\frac{\|\nabla \phi\|^{2}}{\phi^{2}}\right),
$$

donde

$$
\begin{aligned}
R_{\phi g_{s}} & =\phi^{-1}\left[R_{g_{s}}+\not 2(m-1) \frac{1}{\not 2}\left(\frac{\Delta \phi}{\phi}+\frac{\|\nabla \phi\|^{2}}{\phi^{2}}\right)-(m-2)(m-1)\left\|\frac{1}{2} \frac{\nabla \phi}{\phi}\right\|^{2}\right] \\
& =\phi^{-1}\left[R_{g_{s}}+(m-1) \frac{\Delta \phi}{\phi}-\frac{(m-1)(m-6)}{4} \frac{\|\nabla \phi\|^{2}}{\phi^{2}}\right] .
\end{aligned}
$$

Sob a ação de $G, R_{\phi g_{s}}$, fica

$$
\begin{aligned}
R_{\phi g_{s}} \circ \xi & =(\phi)^{-1} \circ \xi\left[R_{g_{s}} \circ \xi+(m-1)\left(\frac{\Delta \phi}{\phi}\right) \circ \xi-\frac{(m-1)(m-6)}{4}\left(\frac{\|\nabla \phi\|^{2}}{\phi^{2}}\right) \circ \xi\right] \\
& =(\phi \circ \xi)^{-1}\left[R_{g_{s}} \circ \xi+(m-1) \frac{(\Delta \phi) \circ \xi}{(\phi \circ \xi)}-\frac{(m-1)(m-6)}{4} \frac{\|\nabla \phi\|^{2} \circ \xi}{(\phi \circ \xi)^{2}}\right] .
\end{aligned}
$$

Por outro lado, se fizermos $\mathrm{e}^{2 f}=\phi \circ \xi$,

$$
f=\frac{1}{2} \ln (\phi \circ \xi) .
$$


Tomando $\phi=\phi \circ \xi$ em (C.1), teremos

$$
R_{(\phi \circ \xi) g_{s}}=(\phi \circ \xi)^{-1}\left[R_{g_{s}}+(m-1) \frac{\Delta(\phi \circ \xi)}{(\phi \circ \xi)}-\frac{(m-1)(m-6)}{4} \frac{\|\nabla(\phi \circ \xi)\|^{2}}{(\phi \circ \xi)^{2}}\right] .
$$

Vamos mostrar que o gradiente e laplaciano são invariantes por ações isométricas. Seja $(U, \psi)$ uma carta local em $p \in M$ e $\left(\xi(U), \psi \circ \xi^{-1}\right)$ uma carta local em $\xi(p) \in M$, se $y=\xi(x) \in \xi(U)$, para para $x \in U$, temos

$$
\begin{gathered}
\psi(x)=\left(x_{1}(x), \ldots, x_{m}(x)\right), \\
\left(\psi \circ \xi^{-1}\right)(y)=\left(y_{1}(y), \ldots, y_{m}(y)\right),
\end{gathered}
$$

isto é, $x_{i}(x)=y_{i}(\xi(x))$; o diferencial d $\xi$ é um isomorfismo entre $T_{x} M$ e $T_{y} M$,

$$
(\mathrm{d} \xi)_{x}\left(\frac{\partial}{\partial x_{i}}\right)_{x}=\left(\frac{\partial}{\partial y_{i}}\right)_{\xi(x)} .
$$

Para o gradiente, temos

$$
(\nabla \phi) \circ \xi(x)=g^{i j} \frac{\partial}{\partial y_{j}}\left[\phi \circ\left(\psi \circ \xi^{-1}\right)^{-1}\right] \frac{\partial}{\partial y_{i}}
$$

$$
\nabla(\phi \circ \xi)(x)=g^{i j} \frac{\partial}{\partial x_{j}}\left[(\phi \circ \xi) \circ \psi^{-1}\right] \frac{\partial}{\partial x_{i}} .
$$

enquanto o laplaciano fica

$$
(\Delta \phi)(\xi(x))=\frac{1}{\sqrt{\operatorname{det} g(y)}} \frac{\partial}{\partial y_{j}}\left(\sqrt{\operatorname{det} g(y)} g^{i j}(y) \frac{\partial}{\partial y_{i}}\left[\phi \circ\left(\psi \circ \xi^{-1}\right)^{-1}\right]\right)
$$

$\mathrm{e}$

$$
\Delta(\phi \circ \xi(x))=\frac{1}{\sqrt{\operatorname{det} g(x)}} \frac{\partial}{\partial x_{j}}\left(\sqrt{\operatorname{det} g(x)} g^{i j}(x) \frac{\partial}{\partial x_{i}}\left[(\phi \circ \xi) \circ \psi^{-1}\right]\right) .
$$

Como $\xi$ é uma isometria, $\xi^{*} g=g$, daí

$$
g(x)\left(X_{x}, Y_{x}\right)=g(\xi(x))\left((\mathrm{d} \xi)_{x}\left(X_{x}\right),(\mathrm{d} \xi)_{x}\left(Y_{x}\right)\right)=g(y)\left(X_{\xi(x)}, Y_{\xi(x)}\right)=g(y)\left(X_{y}, Y_{y}\right)
$$

para quaisquer campos $X, Y \in T M$, em particular $g^{i j}(x)=g^{i j}(y)$ para todo $i, j$. Além disso, temos

$$
\frac{\partial}{\partial y_{i}}\left[\phi \circ\left(\psi \circ \xi^{-1}\right)^{-1}\right]=\frac{\partial}{\partial y_{i}}\left[\phi \circ \xi \circ \psi^{-1}\right]=\frac{\partial}{\partial x_{i}}\left[\phi \circ \xi \circ \psi^{-1}\right]=\frac{\partial}{\partial x_{i}}\left[(\phi \circ \xi) \circ \psi^{-1}\right],
$$

analogamente,

$$
\frac{\partial^{2}}{\partial y_{i} \partial y_{i}}\left[\phi \circ\left(\psi \circ \xi^{-1}\right)^{-1}\right]=\frac{\partial^{2}}{\partial x_{i} \partial x_{i}}\left[(\phi \circ \xi) \circ \psi^{-1}\right]
$$

Portanto,

$$
(\nabla \phi) \circ \xi(x)=\nabla(\phi \circ \xi)(x) \text { e }(\Delta \phi)(\xi(x))=\Delta(\phi \circ \xi(x)) .
$$

Resta apenas observar que $\left(R_{g(y)}\right) \circ \xi(x)=R_{g(\xi(x))} \circ \xi(x)=R_{\xi^{*} g(y)}(x)=\left(R_{g(x)}\right)(x)$, para concluirmos que

$$
R_{(\phi \circ \xi) g_{s}}=R_{\phi g_{s}} \circ \xi .
$$

como queríamos. 


\section{Referências Bibliográficas}

[1] S. M. Almaraz, An existence theorem of conformal scalar-flat metrics on manifolds with boundary, Pacific J. Math. 248 (2010), no. 1, 1-22.

[2] T. Aubin, Equations differentielles non lineaires et probleme de Yamabe concernant la courbure scalaire, J. Math. Pures Appl. 55 (1976), 269-296.

[3] P. H. Berard, Spectral geometry: direct and inverse problems, Springer-Verlag,Berlin, Heidelberg, New York, 1986.

[4] M. Berger, P. Gauduchon, E. Mazet, Le Spectre d'une Variété e Riemannienne, Lecture Notes in Mathematics 194, Springer-Verlag, Berlin, Heidelberg, New York, 1971.

[5] A. L. Besse, Einstein Manifolds, Reprint of 1987 edition, Springer-Verlag, Berli-Heidelberg, 2008.

[6] R. G. Bettiol, P. Piccione, Bifurcation and local rigidity of homogeneous solutions to the Yamabe problem on spheres, Calc. Var. Partial Differential Equations 47 (2013), no. 3-4, 789-807.

[7] R. G. Bettiol, P. Piccione, G. Siciliano, Deforming Solutions of Geometric Variational Problems with Varying Symmetry Groups Transform. Groups 19, (2014), no.4, 941-968.

[8] R. G. Bettiol, P. Piccione, G. Siciliano, Equivariant Bifurcation in Geometric Variational Problems. Analysis and Topology in Nonlinear Differential Equations. Progress in Nonlinear Differential Equations and Their Applications, 85, (2014), 103-133.

[9] R. G. Bettiol, P. Piccione, Multiplicity of solutions to the Yamabe problem on collapsing Riemannian submersions, Pacific J. Math. 266 (2013), no. 1, 1-21.

[10] W. M. Boothby, An Introduction to Differentiable Manifolds and Riemannian Geometry, Academic Press Inc, London, 1986.

[11] A. Cap, J. Slovák, Parabolic Geometries I, Background and General Theory, American Mathematical Society, Providence, Rhode Island, 2009.

[12] P. Cherrier, Problèmes de Neumann non linéaires sur les variétés riemanniennes, J. Funct. Anal 57, (1984), 154-206.

[13] B. Dacorogna, Introduction to the Calculus of Variations, Imperial College Press, Chapter 1, Section 1.2, 12-16, 2004.

[14] E. D. C. Diaz, A. C. S. Moreira, On the Bifurcation of Solutions of the Yamabe Problem on Product Manifolds with Minimal Boundary. To appear in Advances in Nonlinear Analysis.

[15] M. Disconzi, M. Khuri. Compactness and non-compactness of the Yamabe problem on manifolds with boundary, J. Reine Angew. Math. (2014), DOI 10.1515/crelle-2014-0083. 
[16] A. Einstein Zur allgemeinen Relativitatstheoric, Sitzungsberichte der Preussischen Akademie der Wissenschaften zu Berlin (1915), 831-839.

[17] J.F. Escobar Uniqueness theorems on conformal deformation on metrics, Sobolev inequalities, and an eigenvalue estimate, Comm. Pure Appl. Math. 43 (1990), no. 7, 857-883.

[18] J. F. Escobar, The Yamabe problem on manifolds with boundary, J. Differential Geom. 35 (1992), no.1, 21-84.

[19] J. F. Escobar, Conformal deformation of a Riemannian metric to a scalar flat metric with constant mean curvature of the boundary, Ann. of Math 2 (1992), no.1, 1-50.

[20] J. F. Escobar, Conformal deformation of a riemannian metric to a constant scalar curvature metric with constant mean curvature on the boundary, Indiana Univ. Math. J. 45 (1996), 917-943.

[21] W. Fulton, J. Harris, Representation Theory - A first course, Springer-Verlag, New York, 2004.

[22] V. Guillemin, A. Pollack, Differential Topology, Prentice-Hall, New Jersey, 1974.

[23] Z. Han, Y. Y. Lin, The Yamabe problem on manifolds with boundary: existence and compactness results, Duke Mathematical Journal, 99 (1999), no. 3, 489-541.

[24] D. Hilbert, Die Grundlagen der Physik, Nachrichten der Gesellschaft der Wissenschaften Giittingen, Math.-Phys. Klasse, (1915), 395-407.

[25] M. W. Hirsch, S. Smale, R. L. Devaney, Differential Equations, Dynamical Systems \& an Introduction to Chaos, Elsevier (USA), 2004.

[26] N. Koiso, On the second derivative of the total scalar curvature, Osaka J. Mathc. 16 (1979), $413-421$.

[27] E. Kreyszig, Introductory Functional Analysis with Applications, John Wiley \& Sons, 1978.

[28] S. Lang, Differential Manifolds, Springer-Verlag, 1985.

[29] J. M. Lee, Introduction to Smooth Manifolds, Springer-Verlag, 2003.

[30] L. L. Lima, P. Piccione, M. Zedda, A note on the uniqueness of solutions for the Yamabe problem, Proc. Amer. Math. Soc. 140 (2012), no. 12, 4351-4357.

[31] L. L. Lima, P. Piccione, M. Zedda, On bifurcation of solutions of the Yamabe problem in product manifolds, Annales de l'Institut Henri Poincare (C) Non Linear Analysis 29 (2012) Issue 2, 261-277.

[32] Y. Ollivier, A visual introduction to Riemannian curvatures and some discrete generalizations, Analysis and Geometry of Metric Measure Spaces: Lecture Notes of the 50th Séminaire de Mathématiques Supérieures (SMS), Montréal, 2011, Galia Dafni, Robert McCann, Alina Stancu, eds, AMS (2013).

[33] R. W. Sharpe, Differential Geometry - Cartan's Generalization of Klein's Erlanger Program, Springer-Verlag, 1996.

[34] R. M. Schoen, Conformal deformation of a Riemannian metric to constant scalar curvature, J. Diff. Geom. 20 (1984), 479-495.

[35] R. M. Schoen, Variational theory for the total scalar curvature functional for Riemannian metrics and related topics, Topics in Calculus of Variations (Montecatini Terme 1987), Lecture Notes in Mathematics vol. 1365, Springer-Verlag, 1989, pp 120-154. 
[36] M. A. Shubin, Pseudodifferential Operators and Spectral Theory, Springer-Verlag, 2001.

[37] E. M. Stein, Singular Integrals and Differentiability Properties of Functions, Princeton Math. Ser. 30, Princeton University Press, Princeton, New Jersey, 1970.

[38] S. Janson, Lecture Notes: Tensors and Differential Forms, Uppsala University, Suécia, 2000.

[39] N. Trudinger, Remarks concerning the conformal deformation of Riemannian structures on compact manifolds, Annali Scuola Norm. Sup. Pisa 22 (1968) 265-274.

[40] J. Smoller, A. G. Wasserman, Bifurcation and symmetry-breaking, Invent. Math. 100 (1990), 63-95.

[41] H. Yamabe, On a deformation of Riemannian structures on compact manifolds, Osaka Math J. 12 (1960), 21-37.

[42] E. Zeidler, Nonlinear Functional Analysis and its Applications, IV: Applications to Mathematical Physic, Springer-Verlag, 1988. 